\title{
WestVirginiaUniversity
}

THE RESEARCH REPOSITORY @ WVU

Graduate Theses, Dissertations, and Problem Reports

2016

\section{Investigations into the nature of magnetism in transition-metal- phthalocyanines}

\author{
Zhengjun Wang
}

Follow this and additional works at: https://researchrepository.wvu.edu/etd

\section{Recommended Citation}

Wang, Zhengjun, "Investigations into the nature of magnetism in transition-metal-phthalocyanines" (2016). Graduate Theses, Dissertations, and Problem Reports. 6906.

https://researchrepository.wvu.edu/etd/6906

This Dissertation is protected by copyright and/or related rights. It has been brought to you by the The Research Repository @ WVU with permission from the rights-holder(s). You are free to use this Dissertation in any way that is permitted by the copyright and related rights legislation that applies to your use. For other uses you must obtain permission from the rights-holder(s) directly, unless additional rights are indicated by a Creative Commons license in the record and/ or on the work itself. This Dissertation has been accepted for inclusion in WVU Graduate Theses, Dissertations, and Problem Reports collection by an authorized administrator of The Research Repository @ WVU.

For more information, please contact researchrepository@mail.wvu.edu. 


\title{
INVESTIGATIONS INTO THE NATURE OF MAGNETISM IN TRANSITION-METAL-PHTHALOCYANINES
}

\section{Zhengjun Wang}

\author{
Dissertation submitted to the \\ Eberly College of Arts and Sciences \\ at West Virginia University \\ in partial fulfillment of the requirements for the degree of \\ Doctor of Philosophy \\ in \\ Physics
}

Mohindar S. Seehra, Ph.D., Chair

Leonardo Golubovic, Ph.D.

Alan D. Bristow, Ph.D.

Edward Flagg, Ph.D.

Dimitris Korakakis, Ph.D.

Department of Physics and Astronomy

Morgantown, West Virginia

2016

Keywords: Magnetic molecular semiconductors, Exchange coupling, Magnetic linear chains, Arrhenius magnetic relaxation

Copyright 2016 Zhengjun Wang 


\section{ABSTRACT \\ INVESTIGATIONS INTO THE NATURE OF MAGNETISM IN TRANSITION-METAL-PHTHALOCYANINES}

\section{Zhengjun Wang}

In this dissertation, the nature of magnetism in transition-metal-doped phthalocyanines (TMPc, $\mathrm{TM}=\mathrm{Co}, \mathrm{Ni}, \mathrm{Cu}, \mathrm{V}$, and $\mathrm{Mn}$, chemical formula: $\mathrm{C}_{32} \mathrm{H}_{16} \mathrm{~N}_{8} \mathrm{TM}$ ) is investigated by measuring their magnetization (M) and ac susceptibilities in magnetic fields up to $90 \mathrm{kOe}$ and covering the wide temperature range of $0.5 \mathrm{~K}$ to $300 \mathrm{~K}$. These low temperatures and high magnetic field measurements combined with detailed analysis of the data have provided new information about the nature of magnetism of TMPc. The powder samples used here were structurally characterized using x-ray diffraction and scanning electron microscopy.

TMPc are a class of organic semiconductors with potential applications in spintronics and they are planar molecules with the TM atom at the center bound to four $\mathrm{N}$ atoms and forming a linear chain along the monoclinic b-axis. Because of this symmetry, the ground states of TMPc often violate the Hunds' rules; e.g. the $\mathrm{S}=3 / 2$ state for $\mathrm{d}^{5} \mathrm{Mn}$ (II) in $\beta$ - MnPc, $\mathrm{S}=1 / 2$ state for the $\mathrm{d}^{7} \mathrm{Co}$ (II) in $\beta$-CoPc, and $S=0$ state for the $\mathrm{d}^{8} \mathrm{Ni}$ (II) in NiPc. The magnetic properties of TMPc are also affected by the stack angle $\delta$ between the orientation of the molecular plane and the b-axis, $\delta$ being $65^{\circ}\left(45^{\circ}\right)$ for $\alpha(\beta)$ phase.

For both $\alpha$-CuPc and $\beta$-CuPc, M vs. T data fit well with the Bonner-Fisher model for $S=1 / 2$ antiferromagnetic (AFM) Heisenberg linear chain yielding the $\mathrm{Cu}^{2+}-\mathrm{Cu}^{2+}$ exchange constant $\mathrm{J}_{\mathrm{ex}} / \mathrm{k}_{\mathrm{B}}=$ $1.7 \mathrm{~K}(-0.2 \mathrm{~K})$ for $\alpha-\mathrm{CuPc}(\beta-\mathrm{CuPc})$. This order of magnitude difference in $\mathrm{J}_{\mathrm{ex}} / \mathrm{k}_{\mathrm{B}}$ is likely due to the smaller $\mathrm{Cu}^{2+}-\mathrm{Cu}^{2+}$ distance in $\alpha$-phase than in $\beta$-phase. The $\mathrm{M}$ vs. $\mathrm{H}$ at $2 \mathrm{~K}$ and $5 \mathrm{~K}$ data are interpreted including the effect of the exchange coupling.

For $\beta$-CoPc, the predicted peak in $\chi$ vs. $\mathrm{T}$ data by Bonner-Fisher model was observed at $\mathrm{T}_{\max }=$ $1.9 \mathrm{~K}$ for the first time yielding the Heisenberg exchange constant $\mathrm{J}_{\mathrm{ex}} / \mathrm{k}_{\mathrm{B}}=-1.5 \mathrm{~K}$ between the $\mathrm{Co}^{2+}-\mathrm{Co}^{2+}$ ions. The isothermal data of $\mathrm{M}$ vs. $\mathrm{H}$ at $1 \mathrm{~K}$ shows good agreement with the Bonner-Fisher model; $\mathrm{M}$ vs. $\mathrm{H}$ at $5 \mathrm{~K}$ data is interpreted by including the effect of the exchange interaction.

For VOPc (phase II), the analysis of the $\mathrm{M}$ vs. $\mathrm{T}$ data shows $\mathrm{VO}^{2+}$ electronic state with spin $\mathrm{S}=$ $1 / 2$ and $\theta=-0.1$ indicates a very weak AFM exchange coupling between neighboring $\mathrm{VO}^{2+}$ ions which implies the near-perfect paramagnetism of VOPc. This conclusion is also supported by the analysis of the $\mathrm{M}$ vs. $\mathrm{H}$ data at several temperatures. The diamagnetic nature of $\beta$-NiPc corresponding to the $\mathrm{S}=0$ state for the $3 \mathrm{~d}^{8} \mathrm{Ni}(\mathrm{II})$ is confirmed by measurements and analysis of the magnetization with temperature-independent susceptibility $\chi_{D}=-3.38 \times 10^{-7} \mathrm{emu} / \mathrm{gOe}$. The observed ferromagnetic component in the $\beta$-NiPc powder sample is identified with Fe impurity.

For $\beta-\mathrm{MnPc}$ with $\mathrm{S}=3 / 2$, detailed analysis of the $\mathrm{dc}$ and ac susceptibilities leads to the conclusion of the absence of 3-dimensional long range magnetic ordering in this system contrary to previous reports. This conclusion is supported by the Arrott plots and lack of a peak in the ac 
susceptibilities and specific heat near the quoted $\mathrm{T}_{\mathrm{C}} \approx 8.6 \mathrm{~K}$. Instead, $\beta$-MnPc is best described as an Ising-like chain magnet with Arrhenius relaxation of the magnetization governed by an intra-layer ferromagnetic exchange constant $\mathrm{J}_{\mathrm{ex}} / \mathrm{k}_{\mathrm{B}}=2.6 \mathrm{~K}$ and the single ion anisotropy energy constant $|\mathrm{D}| / \mathrm{k}_{\mathrm{B}}$ $=8.3 \mathrm{~K}$. The absence of $3 \mathrm{D}$ long-range ordering is consistent with the measured $|\mathrm{D}|$ being $>\mathrm{J}_{\mathrm{ex}}$. 


\section{DEDICATION}

To my parents Mr. Yubin Wang and Mrs. Lanying Fan

and

my mentor Prof. Mohindar S. Seehra 


\section{ACKNOWLEDGEMENTS}

I would like to take this moment to express my thanks to all those persons whose support and help was important to me while I was pursuing my Ph.D. at West Virginia University.

Dr. Mohindar S. Seehra, you have been an amazing mentor to me in my research and academics. Your knowledge, patience, and guidance enabled me to complete my work successfully. I am very fortunate and honored to be your last Ph.D. student and I thank you again for all your help. I would also like to thank Dr. Dimitris Korakakis for his support and guidance during my initial research on the Indigo thin films. Thanks to my committee members Dr. Leonardo Golubovic, Dr. Alan Bristow, and Dr. Edward Flagg and Dr. Dimitris Korakakis for their time and efforts. I am grateful to the professors in Department of Physics and Astronomy who have taught me physics courses and would like to thank the department office staff and machine shop staff for their assistance. This work would not have been possible without the financial support provided by research grants of Dr. Seehra and the teaching assistantships provided by the Department of Physics and Astronomy.

Thanks to my collaborators: Dr. Eun Sang Choi, Dr. Naresh Dalal, and Dr. Minseong Lee at the National High Magnetic Field Laboratory and Florida State University who helped me to take some low temperature and high magnetic field measurements. I also thank Mr. James Poston of the DOE National Energy Technology Laboratory who helped me to acquire the SEM images for all the samples and Dr. Li Pi at the High Magnetic Field Laboratory, Chinese Academy of Sciences who helped me to take low temperature magnetic measurements on some of the samples. 
I also thank my lab mates for their generous help: Dr. Kelly Pisane, Dr. Vishal Narang, and Usha Geddam.

I would like to thank Dr. Weiqiang Ding at WVU Shared Research Facilities for experimental assistance. Also, Dr. Shi Li at Quantum Design Inc. offers me much help on PPMS and I really appreciate his help.

I think Dr. Larry Halliburton, Dr. Min Ding, Dr. Hanting Zhang, Dr. Hao Ma, Dr. Shih-Houng Young for their guidance and generous help.

I thank all my friends who made my life much easier, especially Disheng Chen, Bingyi Cui, Peng Zheng, Xiaomeng Li, Dr. Chenbo Dong, Dr. Ming Yang, and Dr. Weitao Dai.

I thank Haofan Zheng for his help on editing and formatting the dissertation.

Finally, I would like to thank my parents Mr. Yubin Wang and Mrs. Lanying Fan for giving me a healthy body which enables me to explore this beautiful world. I thank them for their guidance, patience, encouragement, and great support. 


\section{TABLE OF CONTENTS}

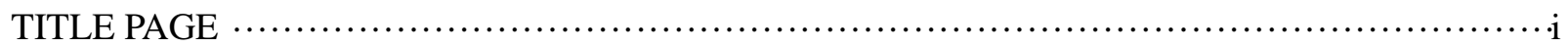

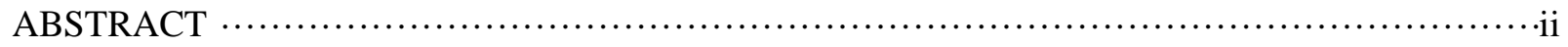

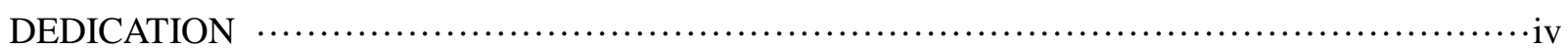

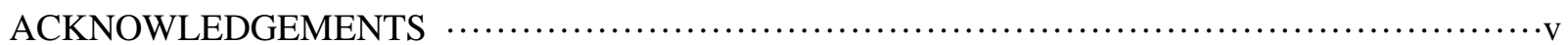

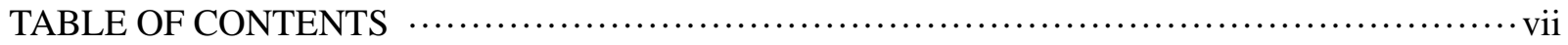

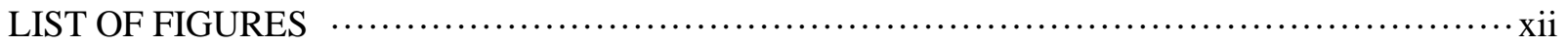

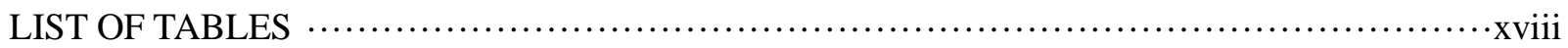

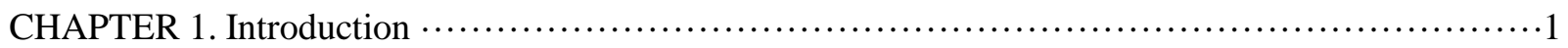

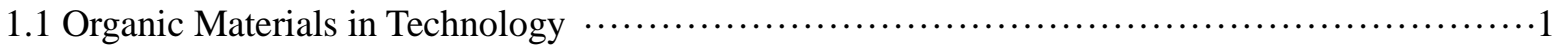

1.2 Introduction to Transition-Metal-Substituted Phthalocyanines (TMPc, TM = V, Fe, Co, Ni, Mn,

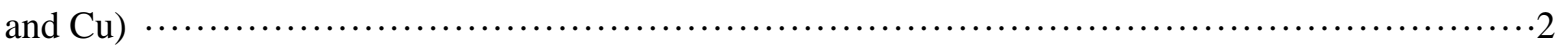

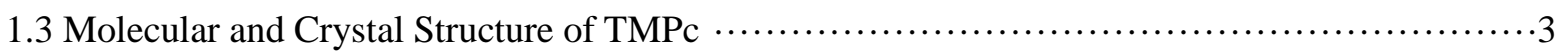

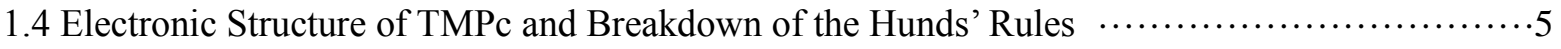

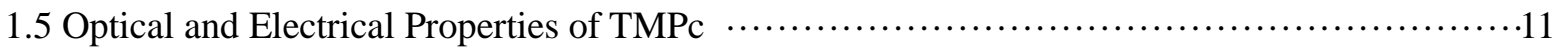

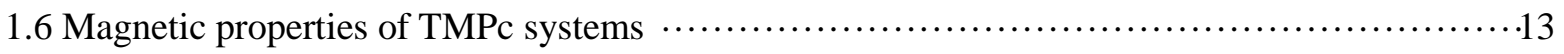

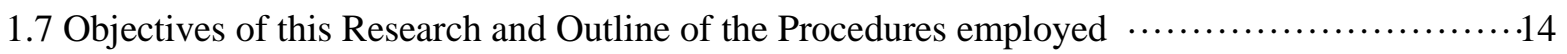

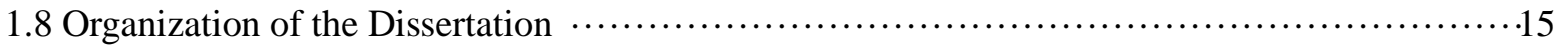

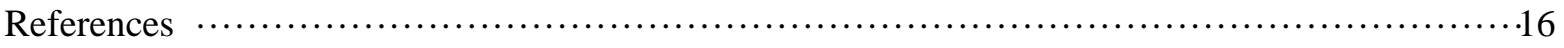

CHAPTER 2. Magnetism: Background and Theoretical Considerations $\ldots \ldots \ldots \ldots \ldots \ldots \ldots \ldots \ldots \ldots \ldots \ldots \ldots \ldots \ldots \ldots \ldots$

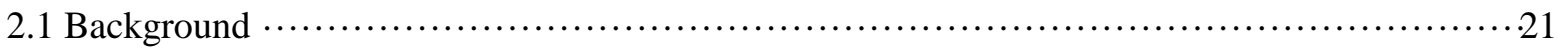

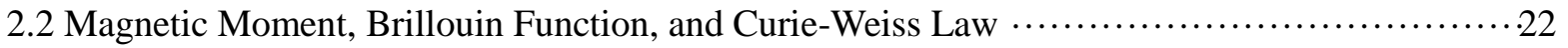

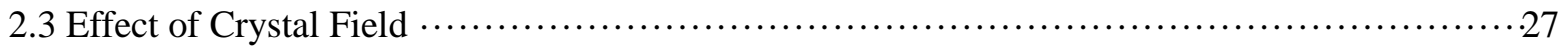

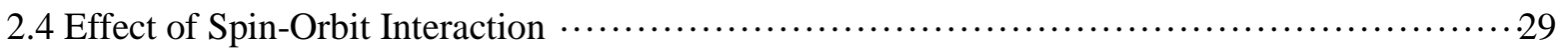

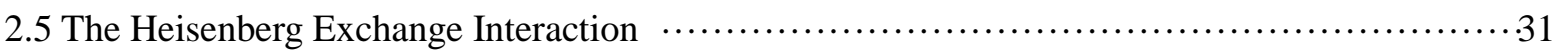

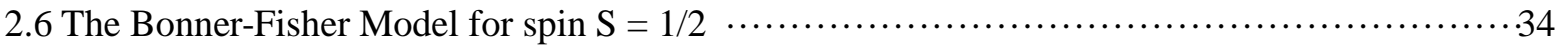

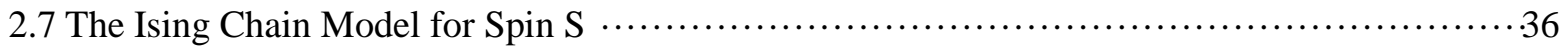




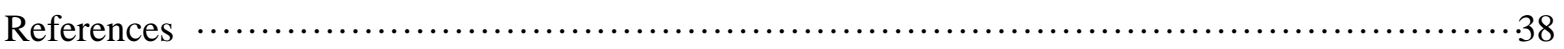

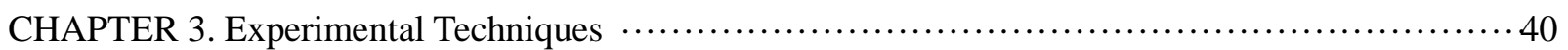

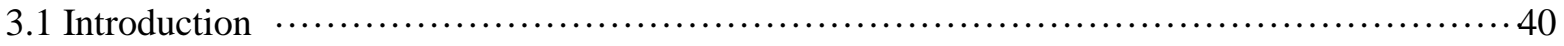

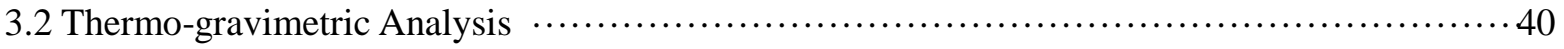

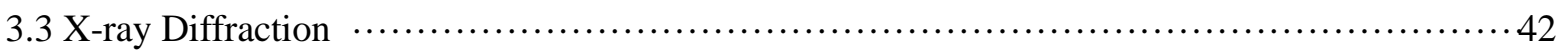

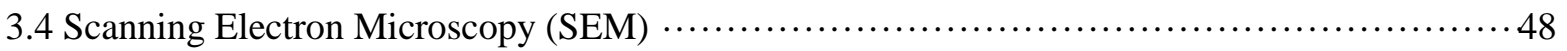

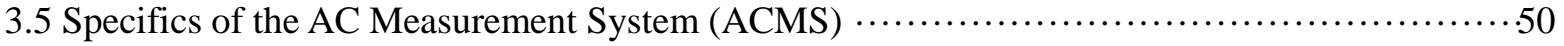

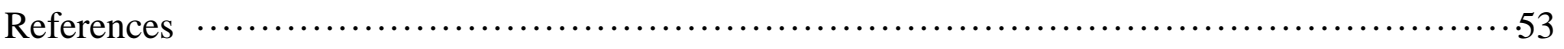

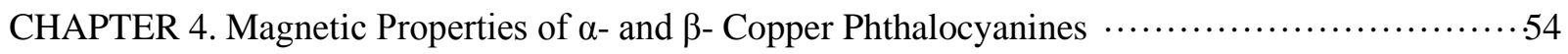

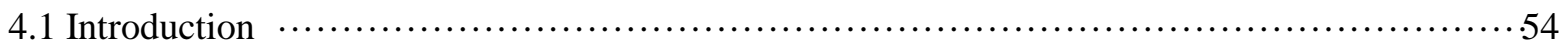

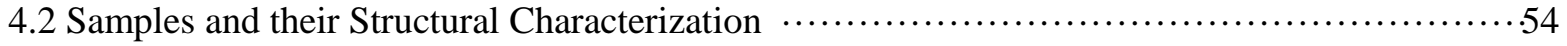

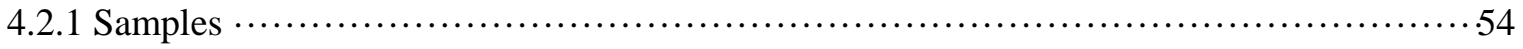

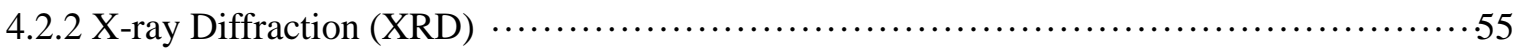

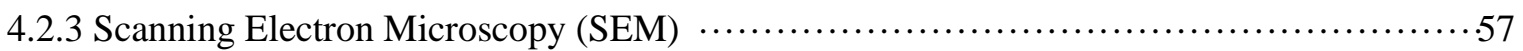

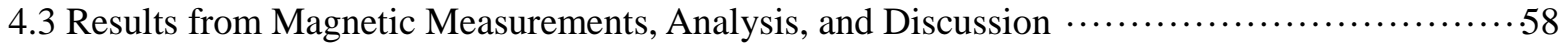

4.3.1 Temperature Dependence of DC Magnetization and the Curie-Weiss Law $\cdots \ldots \ldots \ldots \ldots \ldots . . . \ldots 58$

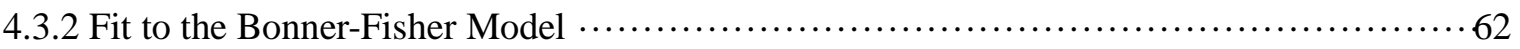

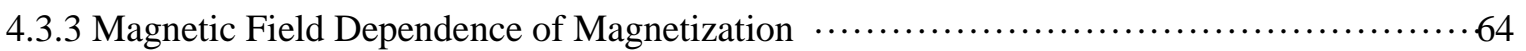

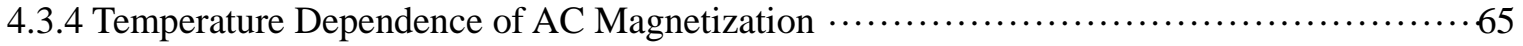

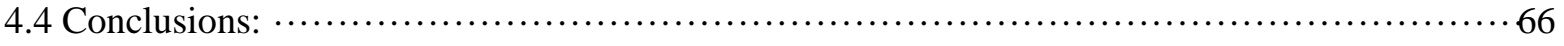

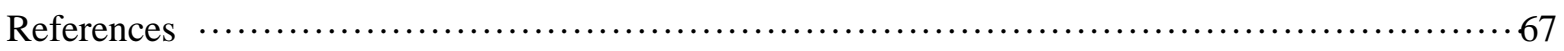

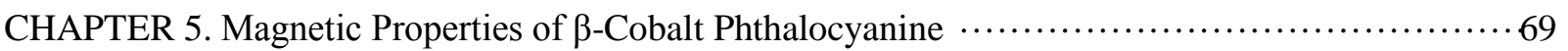

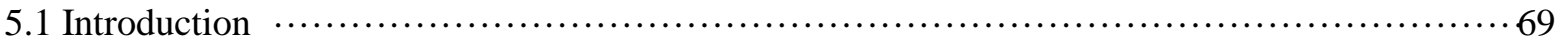

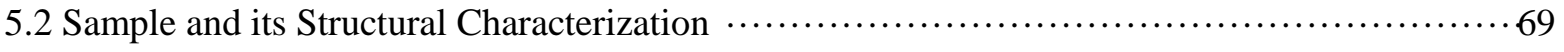

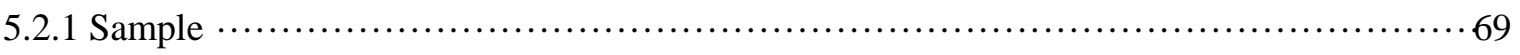

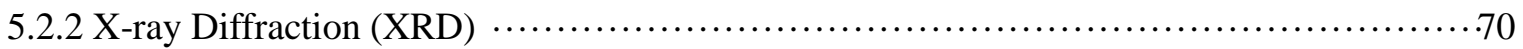

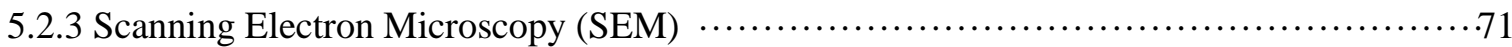




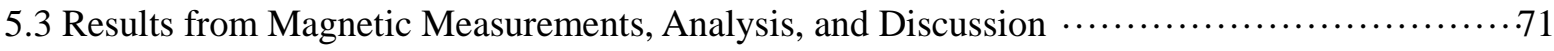

5.3.1. Temperature Dependence of DC Magnetization and the Curie-Weiss Law $. . . \ldots \ldots \ldots \ldots . . .71$

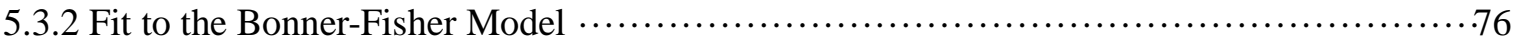

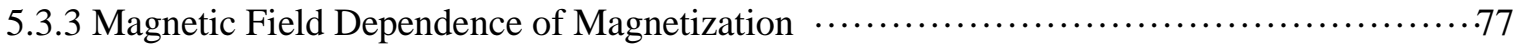

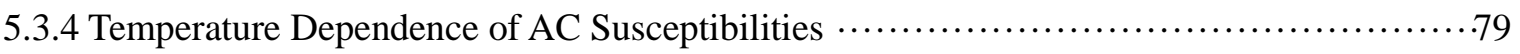

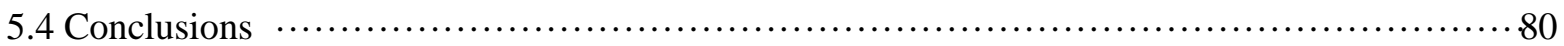

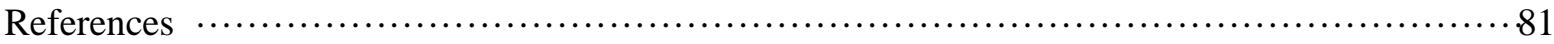

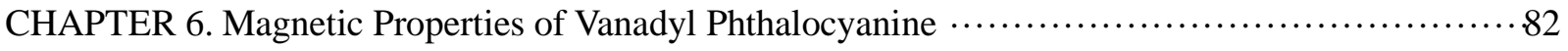

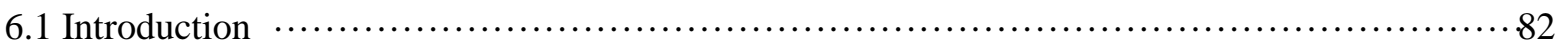

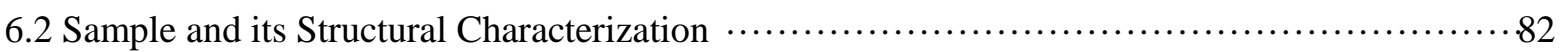

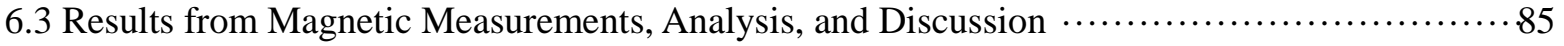

6.3.1 Temperature Dependence of DC Magnetization and the Curie-Weiss Law $\cdots \cdots \ldots \ldots \ldots \ldots . . . .65$

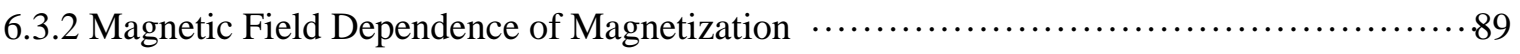

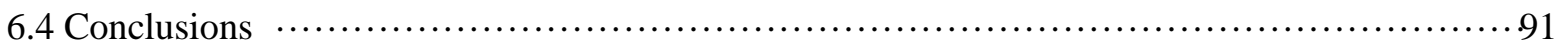

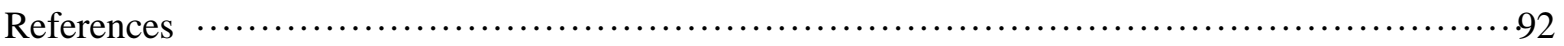

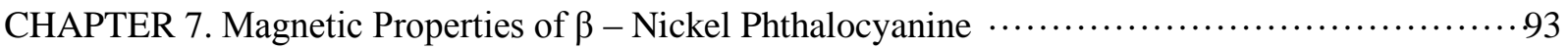

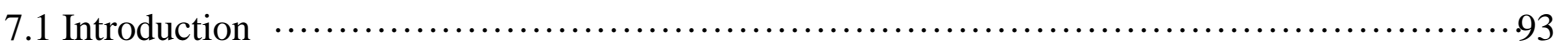

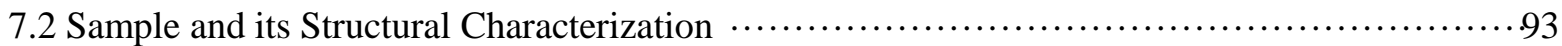

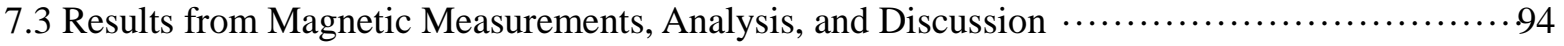

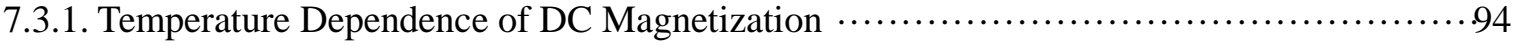

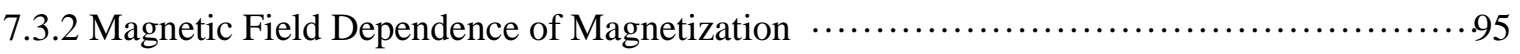

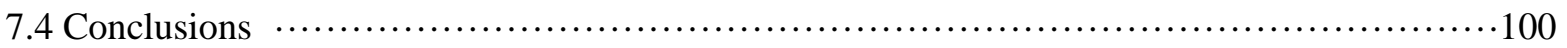

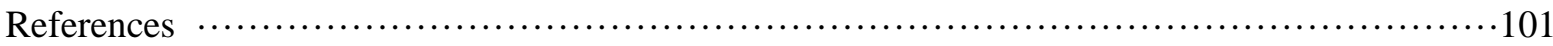

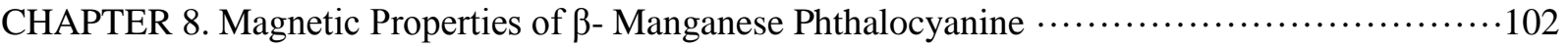

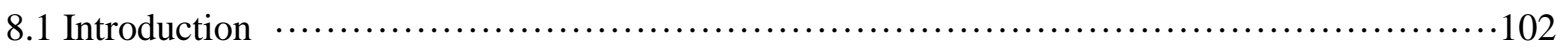

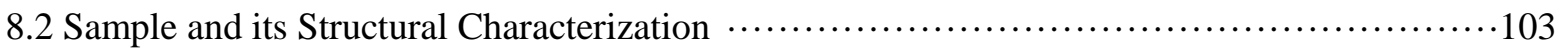

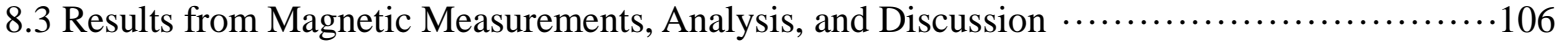


8.3.1. Temperature Dependence of DC Magnetization and the Curie-Weiss Law $\cdots \ldots \ldots \ldots \ldots \ldots . \ldots 106$ 8.3.2. Temperature Dependence of DC Magnetization, Heisenberg Linear Chain, and Ising Linear

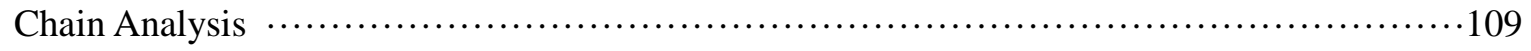

8.3.3. Magnetic Field Dependence of Magnetization and Ising Linear Chain Analysis....... .111

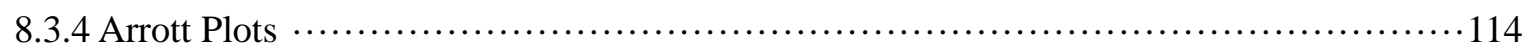

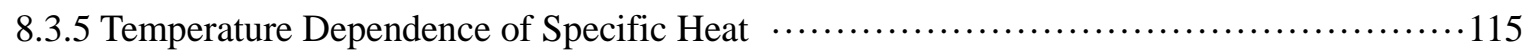

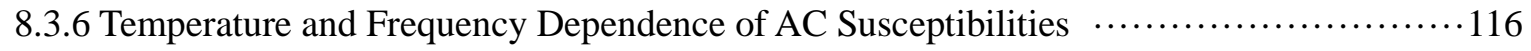

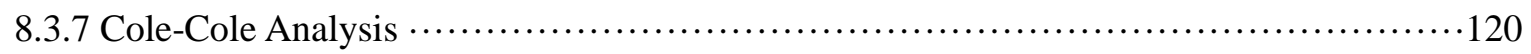

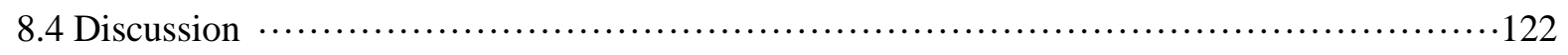

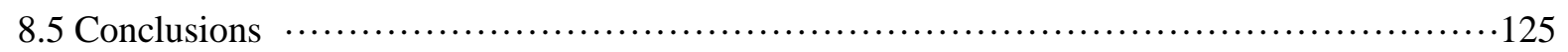

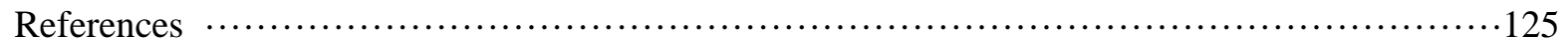

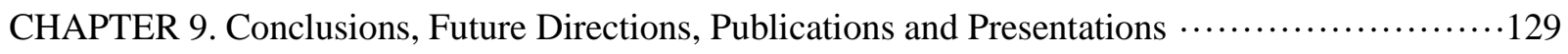

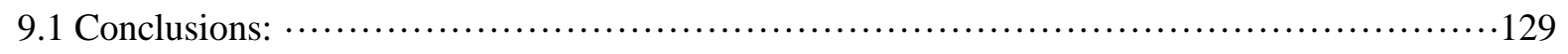

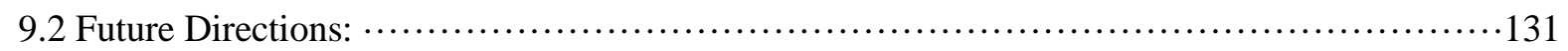

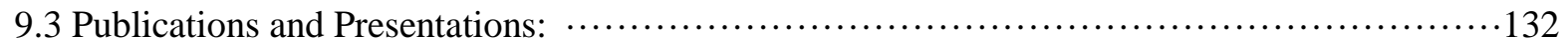

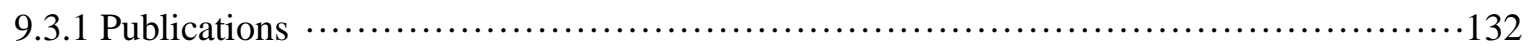

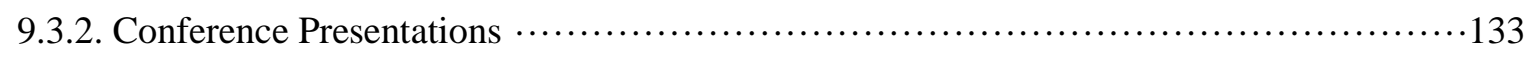

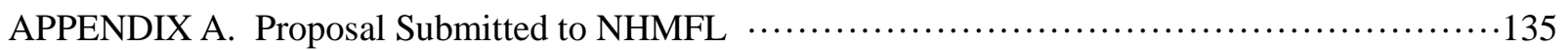

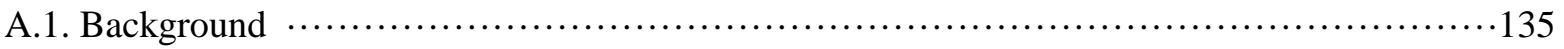

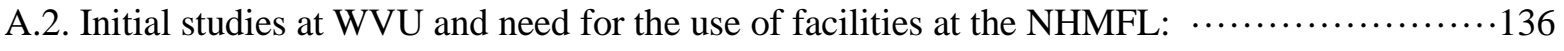

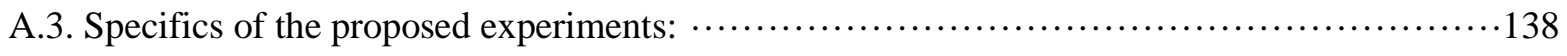

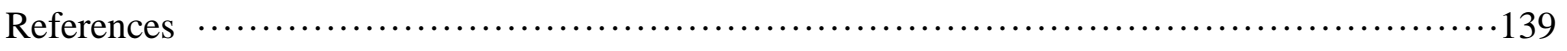

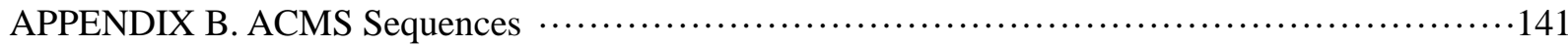

B.1 Zero-field-cool and Field-cool (ZFC-FC) DC Magnetization Measurement from $2 \mathrm{~K}$ to $350 \mathrm{~K}$

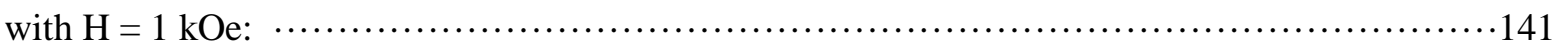

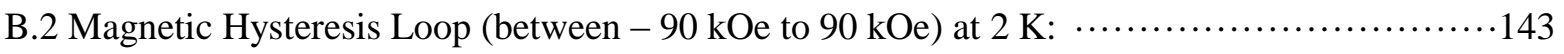

B.3. AC Magnetization Measurement from $2 \mathrm{~K}$ to $20 \mathrm{~K}$ with $\mathrm{H}_{\mathrm{dc}}=0$ Oe and $\mathrm{H}_{\mathrm{ac}}=5 \mathrm{Oe}$, the AC field 
frequencies are: $11.3 \mathrm{~Hz}, 53.1 \mathrm{~Hz}, 101.4 \mathrm{~Hz}, 511.3 \mathrm{~Hz}, 1055.6 \mathrm{~Hz}, 3005.7 \mathrm{~Hz}, 5011.5 \mathrm{~Hz}, 7092.4 \mathrm{~Hz}$,

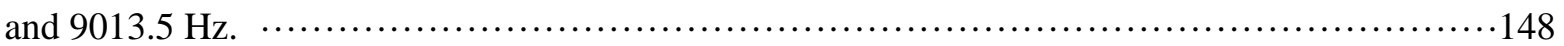

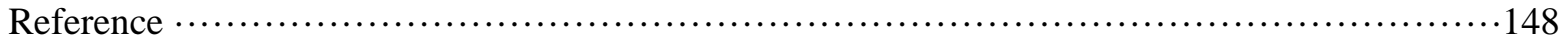




\section{LIST OF FIGURES}

Fig. 1.1 Molecular model (a), molecular arrangement (b), and unit cells (c and d) of $\alpha$-TMPc and $\beta$-TMPc (adapted in part from Ref. 14). The red lines in the unit cell depict the orientation of the molecular plane and $\delta$ is the stack angle. The unit cell parameters of the various TMPc are listed in Table 1.1..3

Fig. 1.2 3d electron orbitals (adapted from http://socratic.org/ [25]) 6

Fig. 1.3 Schematic representation of the TMPc molecular geometry alongside a simplified and general view of the metal $3 \mathrm{~d}^{\mathrm{n}}$ electronic levels according to ligand field theory (top left corner). The molecule is planar, and the $\mathrm{z}$-axis is perpendicular to the molecular plane (adapted from [26]) ......... 8

Fig. 1.4 Two variations of the electron ground state of $\mathrm{Mn}^{2+}$ in $\beta-\mathrm{MnPc}$, both with $\mathrm{S}=3 / 2$ [26].............9

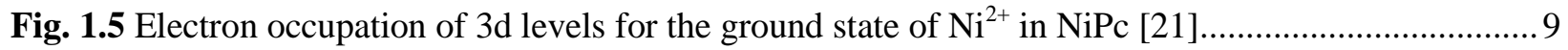

Fig. 1.6 Electron occupation of $3 \mathrm{~d}$ levels for the ground state of $\mathrm{Co}^{2+}$ in $\beta$-CoPc [21] .......................... 10

Fig. 1.7 Electron occupation of $3 \mathrm{~d}$ levels for the ground state of $\mathrm{Cu}^{2+}$ in $\alpha-\mathrm{CuPc}$ and $\beta-\mathrm{CuPc}[21] \ldots \ldots \ldots 11$

Fig. 1.8 Optical absorption spectrum of $\beta-\mathrm{CuPc}$ (adapted from [29]) …............................................ 11

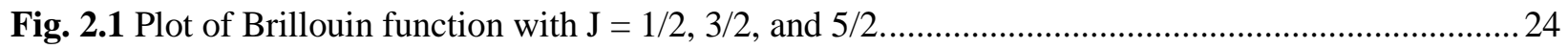

Fig. 2.2 Variation of normalized magnetization with normalized magnetic field, Eq. (2.12)..................26

Fig. 2.3 Splitting of the $3 \mathrm{~d}^{1}$ electron energy level due to cubic field $(\Delta)$ and tetragonal field $(\delta) \ldots \ldots \ldots \ldots . . .28$

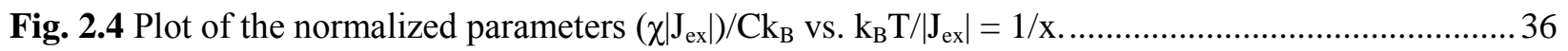

Fig. 2.5 Plot of $\left(\chi\left|\mathrm{J}_{\mathrm{ex}}\right|\right) / \mathrm{Ck}_{\mathrm{B}}$ vs. $\mathrm{k}_{\mathrm{B}} \mathrm{T} /\left|\mathrm{J}_{\mathrm{ex}}\right|=1 / \mathrm{x}$ for Ising chain with spin $\mathrm{S}=1 / 2$ antiferromagnetic coupled system. .37

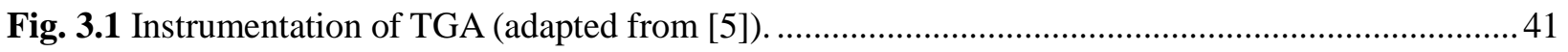

Fig. 3.2 A photograph of the commercial Q50 TGA system used for experiments. ................................ 42

Fig. 3.3 Schematic diagram of X-ray tube (adapted from [7]). ....................................................... 44

Fig. 3.4 Intensity vs. wavelength of X-rays for three applied voltages (adapted from [8]) …..................45

Fig. 3.5 Energy level involved in CuK X-ray emission (adapted from [7]). ......................................... 46

Fig. 3.6 Diffraction of X-rays from two parallel containing atoms (adapted from [9])............................46

Fig. 3.7 Schematic diagram of the key components of SEM systems (adapted from [10])..................... 49

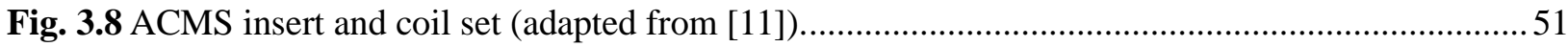


Fig. 4.1 Plots of weight vs. time (a) and weight vs. temperature (b) of $\alpha$-CuPc powder sample during the annealing process. The temperature of sample was varied from $20^{\circ} \mathrm{C}$ to $350{ }^{\circ} \mathrm{C}$ at a heating rate of $10{ }^{\circ} \mathrm{C} / \mathrm{min}$ in flowing $\mathrm{N}_{2}$ gas, then kept constant at $350{ }^{\circ} \mathrm{C}$ for three hours. .55

Fig. 4.2 XRD patterns of $\alpha$-CuPc and $\beta$-CuPc powder sample with Miller indices of major lines shown. No impurity lines were observed in either case. .56

Fig. 4.3 Scanning electron micrographs for (a) $\alpha-\mathrm{CuPc}$ and (b) $\beta$-CuPc. The length scale bars are $100 \mathrm{~nm}$ and $1 \mu \mathrm{m}$ for (a) and (b), respectively. .57

Fig. 4.4 Magnetic susceptibility $\chi$ vs. temperature $\mathrm{T}$ data for $\alpha$-CuPc and $\beta$-CuPc in $\mathrm{H}=1 \mathrm{kOe}$. .57

Fig. 4.5 Magnetic susceptibility $\chi$ vs. $1 / \mathrm{T}$ plots for $\alpha-\mathrm{CuPc}$ and $\beta$-CuPc. The solid lines are linear fits with the intercepts giving $\chi_{\mathrm{o}}=-2.96 \times 10^{-6}\left(-2.70 \times 10^{-6}\right) \mathrm{emu} / \mathrm{gOe}$ for $\alpha-\mathrm{CuPc}(\beta-\mathrm{CuPc})$.

Fig. 4.6 Plots of $\left(\chi-\chi_{0}\right)^{-1}$ vs. T for (a) $\alpha$-CuPc and (b) $\beta$-CuPc. The solid lines are linear fits representing the $\mathrm{CW}$ law with $\mathrm{C}=7.96 \times 10^{-4}\left(7.20 \times 10^{-4}\right)$ emu-K/gOe and $\theta=-2.3 \mathrm{~K}(-0.2 \mathrm{~K})$ for $\alpha-\mathrm{CuPc}$ $(\beta-\mathrm{CuPc})$.

Fig. 4.7 Magnetic susceptibility $\chi$ vs. temperature T for $\alpha-\mathrm{CuPc}$ and $\beta$-CuPc. The solid lines are fits to the modified Curie-Weiss law with the fitting parameters listed in the caption to Fig. 4.6.

Fig. 4.8 Data of $\chi$ vs. T of Fig. 3.5 for the $\alpha$ - and $\beta$-phases of CuPc are fitted to Eq. (3.1) with the solid lines as fits yielding the exchange constant $\mathrm{J}_{\mathrm{ex}} / \mathrm{k}_{\mathrm{B}}=-1.7 \mathrm{~K}(-0.2 \mathrm{~K})$ for $\alpha(\beta)$ phase. The fit also gives $\mathrm{T}_{\max }=2.2 \mathrm{~K}$ for $\alpha$-phase and calculated $\mathrm{T}_{\max }=0.25 \mathrm{~K}$ for $\beta$-phase (see inset).

Fig. 4.9 Magnetization M vs. magnetic field $\mathrm{H}$ of $\alpha$ - and $\beta$-phases of $\mathrm{CuPc}$ at $2 \mathrm{~K}$ and $5 \mathrm{~K}$. The solid lines represent fits to Eq. (4.5) with $\theta=-2.3 \mathrm{~K}(-0.2 \mathrm{~K})$ for $\alpha(\beta)$-phase.

Fig. 4.10 Temperature dependence of the ac susceptibilities $\chi^{\prime}$ and $\chi^{\prime \prime}$ for $\alpha$-CuPc measured in zero applied static field with $\mathrm{H}_{\mathrm{ac}}=5 \mathrm{Oe}$ at frequency $\mathrm{f}=500 \mathrm{~Hz}, 1000 \mathrm{~Hz}$, and $5000 \mathrm{~Hz}$.

Fig. 5.1 The top panel shows the XRD pattern of $\beta$-CoPc sample with Miller indices of major lines marked; the bottom panel shows the ICDD data of $\beta$-CoPc. 70

Fig. 5.2 A Scanning electron micrograph for the $\beta$-CoPc powder sample .71

Fig. 5.3 Magnetic susceptibility $\chi$ vs. temperature T of $\beta$-CoPc. The inset shows the M vs. H. plot. .72

Fig. 5.4 Plot of magnetic susceptibility $\chi$ vs. $1 / \mathrm{T}$ for $\beta$-CoPc with $\mathrm{H}=10 \mathrm{kOe}$. The solid line is the linear 
fit of the experimental data and its intercept with y axis $(\chi)$ gives $\chi_{0}=-1.8 \times 10^{-6} \mathrm{emu} / \mathrm{gOe}$. .73

Fig. 5.5 Plot of the temperature dependence of the inverse magnetic susceptibility $\left(\chi-\chi_{0}\right)^{-1}$ of $\beta$-CoPc in $\mathrm{H}=10 \mathrm{kOe}$. The solid line is the linear fit representing CW law. .74

Fig. 5.6 Magnetic susceptibility $\chi$ vs. temperature $\mathrm{T}$ of $\beta$-CoPc with $\mathrm{H}=10 \mathrm{kOe}$. The Solid line is fit to the modified Curie-Weiss law: $\chi=\chi_{0}+\mathrm{C} /(\mathrm{T}-\theta)$ with the parameters: $\chi_{0}=-1.8 \times 10^{-6} \mathrm{emu} / \mathrm{gOe}, \mathrm{C}=$ $1.02 \times 10^{-3} \mathrm{emu}-\mathrm{K} / \mathrm{gOe}$, and $\theta=-2.5 \mathrm{~K}$. .75

Fig. 5.7 Magnetic susceptibility $\chi$ vs. temperature $\mathrm{T}$ plot for $\beta$-CoPc. The solid line is fit to the BF model (Eq. 5.3) using $\chi_{\mathrm{o}}=-1.8 \times 10^{-6} \mathrm{emu} / \mathrm{gOe}, \mathrm{C}=1.02 \times 10^{-3} \mathrm{emu}-\mathrm{K} / \mathrm{gOe}$, and $\mathrm{J}_{\mathrm{ex}} / \mathrm{k}_{\mathrm{B}}=-1.5 \mathrm{~K}$. .76

Fig. 5.8 Magnetization $M$ vs. $\mathrm{H}$ data for $\beta$-CoPc at $1 \mathrm{~K}$ (black solid line) and $5 \mathrm{~K}$ (open circles) are compared respectively with the predictions of the Bonner-Fisher model at $0.9 \mathrm{~K}$ (red line) [from Ref. 6] and Eq. (5.5) for $5 \mathrm{~K}$ (blue line). .78

Fig. 5.9 Frequency dependence of the ac susceptibilities $\chi^{\prime}$ and $\chi^{\prime \prime}$ for $\beta$-CoPc measured in zero applied static field with $\mathrm{H}_{\mathrm{ac}}=5$ Oe at several frequencies between $725 \mathrm{~Hz}$ and $5000 \mathrm{~Hz}$. 80

Fig. 6.1 XRD pattern of VOPc powder sample with Miller Indices of the peaks shown. 83

Fig. 6.2 Molecular structure and unit cell of VOPc (phase II). The lower panel shows a view down the b-axis of the unit cell. The blue lines in the unit cell depict the orientation of the molecular planes of VOPc. .84

Fig. 6.3 A Scanning electron micrograph for the VOPc sample. The length of scale bar is $1 \mu \mathrm{m}$. .85

Fig. 6.4 Magnetic susceptibility $\chi$ vs. temperature T of VOPc powder sample. 86

Fig. 6.5 Plot of magnetic susceptibility $\chi$ vs. $1 / T$ for VOPc with $H=1$ kOe. The solid line is the linear fit of the experimental data and its intercept with y axis $(\chi)$ gives $\chi_{\mathrm{o}}=-9.3 \times 10^{-7} \mathrm{emu} / \mathrm{gOe}$.

Fig. 6.6 Plot of temperature dependence of the inverse magnetic susceptibility $\left(\chi-\chi_{0}\right)^{-1}$ of VOPc in $\mathrm{H}=1$ kOe. The solid line is the linear fit representing CW law. 87

Fig. 6.7 Magnetic susceptibility $\chi$ versus temperature $\mathrm{T}$ from $0.5 \mathrm{~K}$ to $300 \mathrm{~K}$ with $\mathrm{H}=1 \mathrm{kOe}$ of VOPc powder sample. The open circles are experimental data and the solid lines are fits to the modified Curie-Weiss law with the parameters: $\chi_{0}=-9.3 \times 10^{-7} \mathrm{emu} / \mathrm{gOe}, \mathrm{C}=6.266 \times 10^{-4} \mathrm{emu}-\mathrm{K} / \mathrm{gOe}$, and $\theta$ $=-0.1 \mathrm{~K}$ and $0 \mathrm{~K}$ as shown. 88 
Fig. 6.8 Magnetization $M$ vs. magnetic field $\mathrm{H}$ data for VOPc powder at $0.5 \mathrm{~K}, 1 \mathrm{~K}, 1.5 \mathrm{~K}, 2 \mathrm{~K}, 5 \mathrm{~K}, 10 \mathrm{~K}$, $25 \mathrm{~K}, 50 \mathrm{~K}, 100 \mathrm{~K}$, and $300 \mathrm{~K}$. Note that the data at $0.5 \mathrm{~K}, 1 \mathrm{~K}$, and $1.5 \mathrm{~K}$ are with $\mathrm{H}$ up to $70 \mathrm{kOe}$; data at other temperatures are with $\mathrm{H}$ up to $90 \mathrm{kOe}$ 90

Fig. 6.9 Plot of magnetization $M$ vs. $\mathrm{H} /(\mathrm{T}-\theta)$ with $\theta=-0.1 \mathrm{~K}$ for $\mathrm{VOPc}$ at $0.5 \mathrm{~K}, 1 \mathrm{~K}, 1.5 \mathrm{~K}, 2 \mathrm{~K}, 5 \mathrm{~K}$, $10 \mathrm{~K}, 25 \mathrm{~K}, 50 \mathrm{~K}, 100 \mathrm{~K}$, and $300 \mathrm{~K}$. The solid line (red color) is fit to Eq. (6.1) with $\mathrm{g}=1.967, \mathrm{M}_{\mathrm{S}}=$ $9.48 \mathrm{emu} / \mathrm{g}$, and $\theta=-0.1 \mathrm{~K}$.

Fig. 7.1 The top panel shows the measured XRD pattern of $\beta$-NiPc sample with Miller indices of major lines marked; the bottom panel shows the expected line positions and intensities according to the ICDD data base of $\beta-\mathrm{NiPc}$. .93

Fig. 7.2 Magnetic susceptibility $\chi$ of $\beta$-NiPc vs. temperature $\mathrm{T}$ with $\mathrm{H}=1 \mathrm{kOe}$. .94

Fig. 7.3 Magnetization $\mathrm{M}$ vs. field $\mathrm{H}$ at temperatures indicated for $\beta$-NiPc plus the sample holder......... 95

Fig. 7.4 Magnetization $\mathrm{M}$ vs. field $\mathrm{H}$ at several temperatures for just the sample holder. .96

Fig. 7.5 The magnetization $\mathrm{M}$ vs. field $\mathrm{H}$ data just for $\beta-\mathrm{NiPc}$ at temperatures shown on the plot. 97

Fig. 7.6 Plot of magnetization $\mathrm{M}$ vs. $\mathrm{H}$ for $\beta$-NiPc sample at $\mathrm{T}=300 \mathrm{~K}$. The solid line is the linear fit of the experimental data for higher $\mathrm{H}$ and the slope gives $\chi_{\mathrm{D}}=-3.38 \times 10^{-7} \mathrm{emu} / \mathrm{gOe}$. .98

Fig. 7.7 Temperature dependence of the ferromagnetic component $\mathrm{M}_{\mathrm{FM}}$ and diamagnetic component $\mathrm{M}_{\mathrm{D}}$ for $\beta$-NiPc. The dotted lines connecting the data points are visual guides. .98

Fig. 7.8 Hysteresis loop measured between $+90 \mathrm{kOe}$ to $-90 \mathrm{kOe}$ at $2 \mathrm{~K}$ for $\beta$-NiPc. The inset is the zoom of the low-field region of the hysteresis loop at $2 \mathrm{~K}$

Fig. 7.9 Coercivity $\mathrm{HC}$ vs. temperature $\mathrm{T}$ at $2 \mathrm{~K}, 5 \mathrm{~K}, 10 \mathrm{~K}, 25 \mathrm{~K}, 50 \mathrm{~K}$, and $100 \mathrm{~K}$ for $\beta$-NiPc. 100

Fig. 8.1 (a) XRD pattern of the MnPc powder sample with Miller indices of major lines shown. The stick diagram in (b) shows the relative intensities of the lines according to the ICDD card (\#02-063-3894) for $\beta-\mathrm{MnPc}$.

Fig. 8.2 Molecular model, unit cell, and molecular arrangement of $\beta$-MnPc. The red lines in the unit cell depict the orientation of the molecular planes of $\beta$-MnPc. The green arrows show the direction of magnetic moment perpendicular to the molecular plane, the discussion on which is given in the text. 
Fig. 8.3 The plot of $\mathrm{w} \cos \theta$ versus $\sin \theta$ using prominent Bragg lines of $\beta-\mathrm{MnPc}$ to fit the Williamson-Hall equation: $\mathrm{w} \cos \theta=(0.9 \lambda / \mathrm{D})+\eta \sin \theta$. 105

Fig. 8.4 A scanning electron micrograph for $\beta$-MnPc. The length of scale bar is $1 \mu \mathrm{m}$. 106

Fig. 8.5 Magnetic susceptibility $\chi=M / H$ vs. temperature for $\beta$-MnPc. The inset is the plot of $d \chi / d T$ versus $\mathrm{T}$ data for the $\mathrm{H}=1 \mathrm{kOe}$ data showing a peak at $9 \mathrm{~K}$. 107

Fig. 8.6 Plot of magnetic susceptibility $\chi$ vs. $1 / \mathrm{T}$ for $\beta$-CoPc with $\mathrm{H}=50$ Oe. The solid line is the linear fit of the experimental data and its interception with y axis $(\chi)$ gives $\chi_{0}=-4.83 \times 10^{-6} \mathrm{emu} / \mathrm{gOe} . \ldots . .108$

Fig. 8.7 Magnetic susceptibility $\chi$ vs. T with $\mathrm{H}=50$ Oe for $\beta$-MnPc where the "intrinsic" represents data corrected for demagnetizing factors. Solid line A and the inset are fits to the modified Curie-Weiss law with $\chi_{\mathrm{o}}=-4.83 \times 10^{-6} \mathrm{emu} / \mathrm{gOe}, \mathrm{C}=4.40 \times 10^{-3} \mathrm{emu}-\mathrm{K} / \mathrm{gOe}$, and $\theta=12.3 \mathrm{~K}$. Solid line $\mathrm{B}(\mathrm{C}$ ) is fit to the Ising (Heisenberg) linear chain model with exchange constant $\mathrm{J}_{\mathrm{ex}} / \mathrm{k}_{\mathrm{B}}=2.6 \mathrm{~K}$. 108

Fig. 8.8 $\ln (\chi \mathrm{T} / \mathrm{C})$ vs. $1 / \mathrm{T}$ for $\beta-\mathrm{MnPc}$. The solid line is the linear fit to Eq. (8.2) with $\mathrm{J}_{\mathrm{ex}} / \mathrm{k}_{\mathrm{B}}=2.6 \mathrm{~K} \ldots \ldots 111$

Fig. 8.9 Solid line A is the calculated curve using Eq. (8.3). Curve C represent our experimental data taken at $2 \mathrm{~K}$ on the powder sample of $\beta$-MnPc and normalized to $\mathrm{M}_{\mathrm{S}}=31 \mathrm{emu} / \mathrm{g}$ determined in the limit $1 / \mathrm{H} \rightarrow 0$ (left inset). Data represented by curve $\mathrm{B}$ is the experimental data of $\mathrm{M}$ vs. $\mathrm{H}$ at $4.2 \mathrm{~K}$ for single crystal of $\beta-\mathrm{MnPc}$ along the easy axis and taken from the paper by Mitra et al. [5].The right inset is the zoom of the low-field region of the hysteresis loop.

Fig. 8.10 Domain structure in $\beta$-MnPc reproduced from the paper by Awaga et al. [6]. The applied magnetic field is along the b-axis (black arrow), the two green arrows show the moments on the neighboring chains. (a) Four kinds of magnetic domains at zero field. (b) Single domain under the magnetic field. (c) Rotation of the magnetic moments toward the magnetic field.

Fig. 8.11(a) Magnetization $\mathrm{M}$ vs. magnetic field $\mathrm{H}$ for $\beta-\mathrm{MnPc}$ measured in $1 \mathrm{~K}$ intervals between $2 \mathrm{~K}$ and $11 \mathrm{~K}$; (b) Data of (a) plotted as $\mathrm{H} / \mathrm{M}$ vs. $\mathrm{M}^{2}$. 114

Fig. 8.12 Temperature dependence of total heat capacity (A), estimated lattice contribution (B), and magnetic contribution to the heat capacity $(\mathrm{C})$ of pristine $\beta$-MnPc. The lattice contribution to the heat capacity was estimated from that of $\beta$-NiPc in which $\mathrm{Ni}^{2+}$ has non-magnetic configuration. 115

Fig. 8.13 Frequency dependence of the ac susceptibilities $\chi^{\prime}$ and $\chi^{\prime \prime}$ for $\beta$-MnPc measured in zero applied 
static field with $\mathrm{H}_{\mathrm{ac}}=5 \mathrm{Oe}$ at several frequencies between $10 \mathrm{~Hz}$ and $5000 \mathrm{~Hz}$

Fig. 8.14 Temperature dependence of the ac susceptibilities $\chi^{\prime}$ and $\chi^{\prime \prime}$ for $\beta-\mathrm{MnPc}$ in $\mathrm{H}_{\mathrm{dc}}=0$ Oe (a) and $\mathrm{H}_{\mathrm{dc}}$ $=200 \mathrm{Oe}(\mathrm{b})$ with $\mathrm{H}_{\mathrm{ac}}=5$ Oe at frequency of $100 \mathrm{~Hz}$.

Fig. 8.15 Plot of the blocking temperature $T_{B}$ vs. fm with the solid curves fits to Eq. (8.8) with the parameters of the fits shown. The closed circles and squares are data points.

Fig. 8.16 Cole-Cole diagram at various temperatures from $3 \mathrm{~K}$ to $4 \mathrm{~K}$ for $\beta$-MnPc. The solid lines represent least-squares fits to Eq. (8.11) with the magnitudes of the various parameters determined from the fits listed in the figure.

Fig. 8.17 Determination of $\tau_{0}$ and the activation energy for $\beta$-MnPc from the $\ln \tau$ vs. $1 /$ T plot 122

Fig. A.1 The data of $\chi$ vs. T for the $\alpha$ - and $\beta$-phase of CuPc are fitted to Bonner-Fisher model with the solid lines as fits yielding the exchange constant $\mathrm{J}_{\mathrm{ex}} / \mathrm{k}_{\mathrm{B}}=3.4 \mathrm{~K}(0.4 \mathrm{~K})$ for $\alpha(\beta)$ phase. The fit also gives $\mathrm{T}_{\max }=2.2 \mathrm{~K}$ for $\alpha$-phase and calculated $\mathrm{T}_{\max }=0.25 \mathrm{~K}$ for $\beta$-phase (see inset)

Fig. A.2 Magnetic susceptibility $(\chi)$ vs. temperature (T) of $\alpha-\mathrm{CuPc}, \mathrm{MnPc}, \mathrm{FePc}$ and $\mathrm{CoPc}$ measured in the applied magnetic field of $1 \mathrm{kOe}$.

Fig. A.3 Magnetization (M) vs. magnetic field (H) data of $\alpha-\mathrm{CuPc}, \mathrm{MnPc}, \mathrm{CoPc}$, and FePc at 2 K. Note the lack of saturation of $\mathrm{M}$ even at $90 \mathrm{kOe}$. 138 


\section{LIST OF TABLES}

Table 1.1 Lattice parameters for $\alpha-\mathrm{CuPc}, \beta-\mathrm{CuPc}, \beta-\mathrm{CoPc}, \beta-\mathrm{MnPc}$, and $\beta$-NiPc................................5

Table 1.2 Electronic configuration, basic level, spin, and effective magneton numbers (p) for iron group

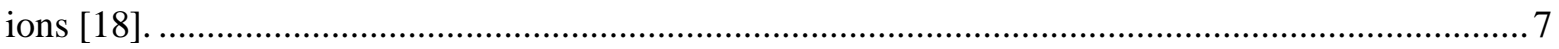

Table 1.3 Electrical conductivity of TMPc thin films at room temperature......................................... 12

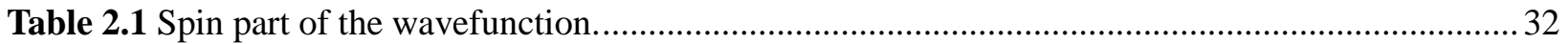

Table 9.1 Major results of this dissertation. Notice that AFM, DM, and FM represent antiferromagnetic,

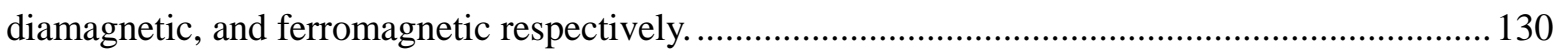




\section{CHAPTER 1.}

\section{Introduction}

\subsection{Organic Materials in Technology}

For the past several decades, organic materials including small molecules and polymers have been of great interest for their fundamental physical properties as well as for their applications in various electronic devices. The main advantages of organic materials compared to their inorganic counterparts are the compatibility with flexible substrates and low-temperature, low-cost fabrication processes such as thermal evaporation, spin coating, and printing. Also, organic materials can be deposited on flexible, light-weight, and large area substrates which offers the possibilities of practical applications. Moreover, both physical and chemical properties can be tailored by manipulating ligands or functional groups, offering the way for scientists and engineers to design molecules with desired properties. A number of organic materials $\left[\mathrm{Alq}_{3}\left(\mathrm{Al}\left(\mathrm{C}_{9} \mathrm{H}_{6} \mathrm{NO}_{3}\right)_{3}\right)\right.$, Pentacene, $\mathrm{CuPc}$ and indigo to name a few] have been used to fabricate various organic electronic devices such as organic spintronic devices, organic solar cells (OSC), organic light emitting diodes (OLED), organic thin film transistors (OTFT), organic memory devices, and organic bioelectronics devices [1-3]. On the other hand, although crystalline inorganic materials (e.g. metals and ionic crystals) have been well understood theoretically, physical properties of organic materials such as crystal structure, band structure, magnetic, optical, and electrical properties still require a lot of theoretical and experimental research. Thus, organic materials offer scientists great opportunities to develop both theory and experimental techniques. 


\subsection{Introduction to Transition-Metal-Substituted Phthalocyanines (TMPc, TM = V, Fe, Co, Ni, Mn, and $\mathrm{Cu})$}

Metal Phthalocyanines (MPc, chemical formula: $\mathrm{C}_{32} \mathrm{H}_{16} \mathrm{~N}_{8} \mathrm{M}$ ) were successfully synthesized in the early $20^{\text {th }}$ century and primarily used as pigments since then. Due to their strong absorption in the visible region and high fastness to light, weathering and numerous solvents, MPcs are used in nearly all fields of pigment industry, such as coatings, coloration of plastics, textile printing and so on. Over seventy MPc have been synthesized by placing different metal ions in the central cavity of Pc and among all the MPc, Copper Phthalocyanines $(\mathrm{CuPc})$, including the chloro and bromo derivatives (important green organic pigments), are the predominant pigments.

Driven by the pigment industry, much emphasis was placed on the synthesis of different MPc molecules at the early stage without attention to their physical properties, and molecular and crystal structure. This did not change until Linstead et al. at the University of London proposed the molecular structure of MPc in 1930s [4-5]. This was soon confirmed using X-ray diffraction (XRD) by Robertson [6-7]. Other fundamental physical properties of MPcs such as optical, magnetic, and electrical properties have been investigated since the 1940s. Actually, MPc are one of the key materials for molecular physics due to their simplicity of molecular structure.

If a transition-metal (TM) atom is substituted in the center of the molecule, TMPc carry a magnetic moment because of the unpaired spin of the transition-metal atom. Transition-metal-substituted phthalocyanines (TMPc, $\mathrm{M}=\mathrm{V}, \mathrm{Fe}, \mathrm{Co}, \mathrm{Ni}, \mathrm{Mn}$, and $\mathrm{Cu}$ ) have become materials of great interest recently not only for their unique physical properties but also for potential applications in spintronics and 
optoelectronic devices [8-10]. One such possibility is discussed by Warner et al [10] where the use of $\mathrm{CuPc}$ thin films is proposed for spin-based information technology (spintronics) because of their relatively long population relaxation time $\mathrm{T}_{1}(10 \mu \mathrm{s})$ and phase memory time $\mathrm{T}_{2}(1 \mu \mathrm{s})$ at $80 \mathrm{~K}$.

\subsection{Molecular and Crystal Structure of TMPc}

TMPc are planar molecules with a transition-metal atom bound at the center with four nitrogen atoms, as shown in Fig. 1.1(a). One of the interesting aspects of TMPc is their variety in crystal structure leading to different phases such as $\alpha, \beta, \gamma$, etc. [11]. Different crystal structures have different properties, such as density, melting point, solubility, and color. Besides X-ray diffraction and neutron diffraction, Fourier Transform Infrared Spectroscopy (FTIR) and Electron Spin Resonance (ESR) can also be used to identify the crystal structure for TMPc [12]. This is because polymorphism leads to different inter-molecular interactions in the different crystal lattices leading to specific electronic and vibrational properties for each crystal form [13].

(a)
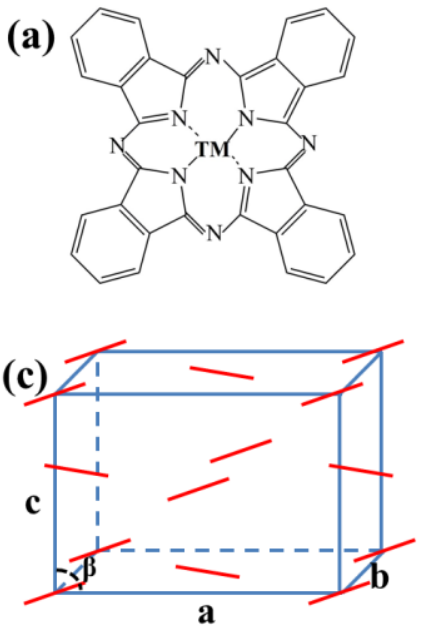

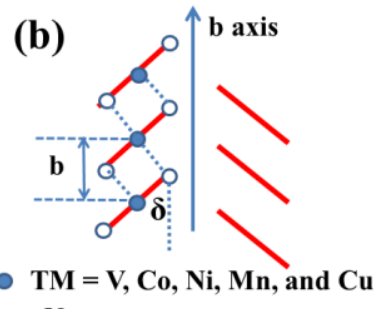

$\circ \mathbf{N}$

(d)

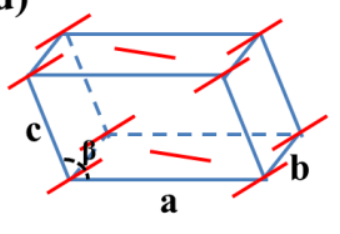

Fig. 1.1 Molecular model (a), molecular arrangement (b), and unit cells ( $c$ and $d)$ of $\alpha$-TMPc and $\beta$-TMPc (adapted in part from Ref. 14). The red lines in the unit cell depict the orientation of the molecular plane and $\delta$ is the stack angle. The unit cell parameters of the various TMPc are listed in Table 1.1 . 
Among these different crystal structures for TMPc, $\alpha$-phase and $\beta$-phase are the most common phases as shown in Fig.1.1 (c) and (d). There are four and two molecules in each unit cell for the $\alpha$-phase and $\beta$-phase, respectively. Usually, the $\beta$-phase TMPc is more stable than the $\alpha$-phase since the latter can be converted to the $\beta$-phase by annealing in $\mathrm{N}_{2}$ environment near $350{ }^{\circ} \mathrm{C}$ [11]. With regard to the nature of magnetism in TMPc, the distance between the nearest neighbor molecules along a-axis or c-axis is more than $1 \mathrm{~nm}$ which is too far for TM atoms to form the direct exchange coupling. However, the distance between the nearest neighboring molecules along the b-axis is only $0.3 \mathrm{~nm}$ to $0.5 \mathrm{~nm}$ which makes direct exchange coupling between TM atoms possible (Fig. 1.1b). Also, TM atoms along the b-axis may form superexchange coupling through nitrogen atom [15]. This is why TMPc are considered as one dimensional (1D) materials along the b-axis magnetically. Besides the distance between the nearest neighboring molecules, the other important parameter of both the $\alpha$-phase and $\beta$-phase for magnetic properties is the magnitude of stack angle $\delta$ (Fig.1.1). In Table 1.1 lattice parameters for $\alpha$-phase Copper Phthalocyanine ( $\alpha$-CuPc), $\beta$-CuPc, $\beta$-CoPc, $\beta$-MnPc, and $\beta$-phase Nickel Phthalocyanine ( $\beta$-NiPc) are listed. The crystal structure for VOPc (phase II) is triclinic, which is different from the above mentioned materials and its lattice parameters are given in Chapter 6. A brief explanation of the space groups $\mathrm{C} 2 / \mathrm{c}$ and $\mathrm{P} 2_{1} / \mathrm{c}$ of Table 1.1 is given as a footnote [16]. 
Table 1.1 Lattice parameters for $\alpha-C u P c, \beta-C u P c, \beta-C o P c, \beta-M n P c$, and $\beta$-NiPc.

\begin{tabular}{|c|c|c|c|c|c|}
\hline & $\alpha-\mathrm{CuPc}$ & $\beta-\mathrm{CuPc}$ & $\beta-\mathrm{CoPc}$ & $\beta-\mathrm{MnPc}$ & $\beta-\mathrm{NiPc}$ \\
\hline $\begin{array}{l}\text { crystal } \\
\text { structure }\end{array}$ & monoclinic & monoclinic & monoclinic & monoclinic & monoclinic \\
\hline space group & $\mathrm{C} 2 / \mathrm{c}$ & $\mathrm{P} 2{ }_{1} / \mathrm{a}$ & $\mathrm{P} 2{ }_{1} / \mathrm{c}$ & $\mathrm{P} 2{ }_{1} / \mathrm{c}$ & $\mathrm{P} 2{ }_{1} / \mathrm{c}$ \\
\hline $\mathrm{a}(\mathrm{nm})$ & 2.592 & 1.9407 & 1.4542 & 1.4576 & 1.490 \\
\hline $\mathrm{b}(\mathrm{nm})$ & 0.379 & 0.479 & 0.4773 & 0.4755 & 0.470 \\
\hline $\mathrm{c}(\mathrm{nm})$ & 2.392 & 1.4628 & 1.9352 & 1.9362 & 1.990 \\
\hline$\beta(\operatorname{deg})$ & 90.4 & 120.56 & 120.82 & 120.7 & 121.15 \\
\hline$\delta(\operatorname{deg})$ & 65 & 45 & 45 & 45 & 45 \\
\hline Reference & [17] & [17] & $\begin{array}{l}\text { ICDD } \\
02-061-3631\end{array}$ & $\begin{array}{l}\text { ICDD } \# \\
02-063-3894\end{array}$ & $\begin{array}{l}\text { ICDD } \\
00-011-0744\end{array}$ \\
\hline
\end{tabular}

\subsection{Electronic Structure of TMPc and Breakdown of the Hunds' Rules}

The Hunds' rules as applied to electrons in a given shell of an atom affirm that electrons will occupy orbitals in such a way that the ground state is characterized by the following [18]: (i) The maximum value of the total spin $\mathrm{S}$ allowed by the exclusion principle; (ii) the maximum value of the 
orbital angular momentum L consistent with this value of $\mathrm{S}$; and (iii) the value of the total angular momentum $\mathrm{J}$ is equal to $|\mathrm{L}-\mathrm{S}|$ when the shell is less than half full and to $\mathrm{L}+\mathrm{S}$ when the shell is more than half full. When the shell is just half full, the application of the first rule gives $\mathrm{L}=0$, so that $\mathrm{J}=\mathrm{S}$. In Table 1.2, the predicted results according to the Hund's rules for the iron group ions with $3 \mathrm{~d}^{\mathrm{n}}$ configurations are listed [18]. As shown in Table 1.2, the spin $\mathrm{S}$ for $\mathrm{Mn}^{2+}, \mathrm{Ni}^{2+}$, and $\mathrm{Co}^{2+}$ should be 5/2, 1 , and $3 / 2$ respectively, according to Hunds' rules. However, the ground state of the TM ions in TMPc often violates Hunds' rules. Examples are the spin $3 / 2$ intermediate state for the $\mathrm{d}^{5} \mathrm{Mn}^{2+}$ in $\beta-\mathrm{MnPc}, \mathrm{S}=0$ state $\mathrm{d}^{8}$ for $\mathrm{Ni}^{2+}$ in $\mathrm{NiPc}$, and $\mathrm{S}=1 / 2$ state for $\mathrm{d}^{7} \mathrm{Co}^{2+}$ in $\beta-\mathrm{CoPc}$ [19-23]. According to ligand-field theory [24], the $3 \mathrm{~d}$ electron energy levels of a TM atom (Fig.1.2) are split if it is placed into a $\mathrm{D}_{4 \mathrm{~h}}$ symmetric environment, which is the case for TMPc. As a result, the $\mathrm{d}_{\mathrm{x}-\mathrm{y}}^{2}{ }^{2}$ level is pushed to much higher energy level (Fig. 1.3) and often is not occupied by electrons, and this causes the breakdown of Hunds' rules for TMPc.

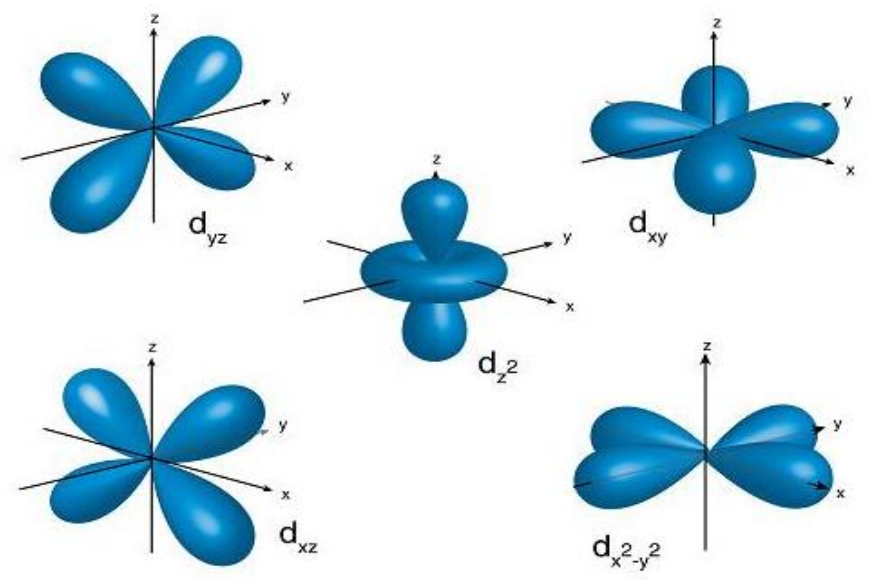

Fig. 1.2 3d electron orbitals (adapted from http://socratic.org/ [25]). 
Table 1.2 Electronic configuration, basic level, spin, and effective magneton numbers (p) for iron group ions [18].

\begin{tabular}{|l|l|l|l|l|l|l|}
\hline Ion & Configuration & Basic level & Spin & $\mathrm{p}(\mathrm{cal})$ & $\mathrm{p}(\mathrm{cal}) \quad$ & $\mathrm{p}(\mathrm{exp})$ \\
\hline $\mathrm{Ti}^{3+}, \mathrm{V}^{4+}$ & $3 \mathrm{~d}^{1}$ & & $\mathrm{~g}[\mathrm{~J}(\mathrm{~J}+1)]^{1 / 2}$ & $\mathrm{~g}[\mathrm{~S}(\mathrm{~S}+1)]^{1 / 2}$ & \\
\hline $\mathrm{V}^{3+}$ & $3 \mathrm{~d}^{2}$ & ${ }^{2} \mathrm{D}_{3 / 2}$ & $1 / 2$ & 1.55 & 1.73 & 1.8 \\
\hline $\mathrm{Cr}^{3+}, \mathrm{V}^{2+}$ & $3 \mathrm{~d}^{3}$ & ${ }^{3} \mathrm{~F}_{2}$ & 1 & 1.63 & 2.83 & 2.8 \\
\hline $\mathrm{Mn}^{3+}, \mathrm{Cr}^{2+}$ & $3 \mathrm{~d}^{4}$ & ${ }^{4} \mathrm{~F}_{3 / 2}$ & $3 / 2$ & 0.77 & 3.87 & 3.8 \\
\hline $\mathrm{Fe}^{3+}, \mathrm{Mn}^{2+}$ & $3 \mathrm{~d}^{5}$ & ${ }^{5} \mathrm{D}_{0}$ & 2 & 0 & 4.90 & 4.9 \\
\hline $\mathrm{Fe}^{2+}$ & $3 \mathrm{~d}^{6}$ & ${ }^{6} \mathrm{~S}_{5 / 2}$ & $5 / 2$ & 5.92 & 5.92 & 5.9 \\
\hline $\mathrm{Co}^{2+}$ & $3 \mathrm{~d}^{7}$ & ${ }^{5} \mathrm{D}_{4}$ & 2 & 6.70 & 4.90 & 5.8 \\
\hline $\mathrm{Ni}^{2+}$ & ${ }^{4} \mathrm{~F}_{9 / 2}$ & $3 / 2$ & 6.59 & 3.8 \\
\hline
\end{tabular}




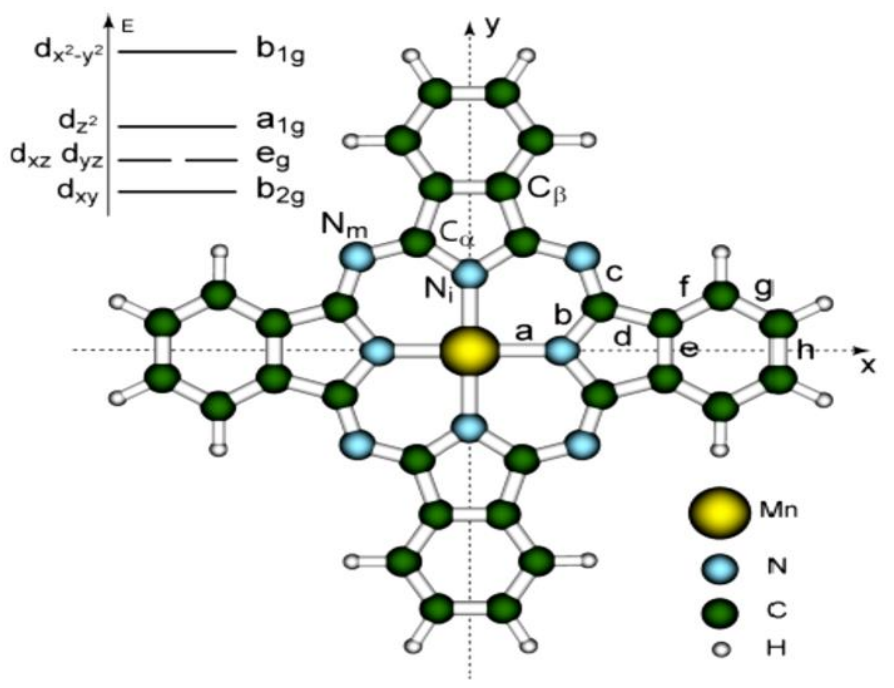

Fig. 1.3 Schematic representation of the TMPc molecular geometry alongside a simplified and general view of the metal $3 d^{n}$ electronic levels according to ligand field theory (top left corner). The molecule is planar, and the z-axis is perpendicular to the molecular plane (adapted from [26]).

For $\beta$-MnPc, Brumboiu et al. performed detailed investigations of the electronic configuration of the ground state by Density Functional Theory (DFT) calculations and Photoelectron Spectroscopy (PES) measurements for both thin film and gas phase [26]. $\mathrm{Mn}^{2+}\left(3 \mathrm{~d}^{5}\right)$ shows $\left[\left(\mathrm{d}_{\mathrm{xy}}\right)^{1}\left(\mathrm{~d}_{\mathrm{xz}}, \mathrm{yz}\right)^{3}\left(\mathrm{~d}_{\mathrm{z}}{ }^{2}\right)^{1}\right]$ as the electronic configuration for the thin film, while in the gas phase it exhibits a mixed electronic configuration with $\left[\left(\mathrm{d}_{\mathrm{xy}}\right)^{1}\left(\mathrm{~d}_{\mathrm{xz}}, \mathrm{yz}\right)^{3}\left(\mathrm{~d}_{\mathrm{z}}^{2}\right)^{1}\right]$ and $\left[\left(\mathrm{d}_{\mathrm{xy}}\right)^{2}\left(\mathrm{~d}_{\mathrm{xz}}, \mathrm{yz}\right)^{2}\left(\mathrm{~d}_{\mathrm{z}}{ }^{2}\right)^{1}\right]$, as shown in Fig. 1.4. As the result, $\mathrm{Mn}^{2+}$ in $\beta-\mathrm{MnPc}$ is in the intermediate spin state $\mathrm{S}=3 / 2$ instead of usual high spin state $\mathrm{S}=5 / 2$ as for example in $\mathrm{MnO}$ [27]. They also demonstrated that the ground state of different kind of samples was determined by many factors such as temperature, preparation method, or intermolecular interactions, which opens up the possibility for manipulation of the electronic configuration for MnPc. 


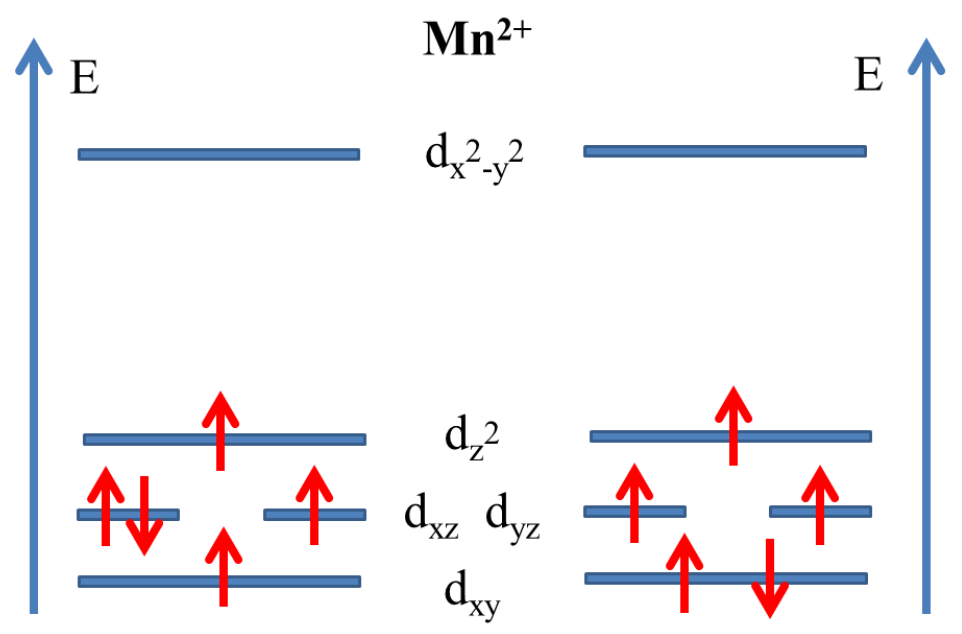

Fig. 1.4 Two variations of the electron ground state of $\mathrm{Mn}^{2+}$ in $\beta-M n P c$, both with $S=3 / 2$ [26].

For NiPc, $\mathrm{Ni}^{2+}\left(3 \mathrm{~d}^{8}\right)$ shows spin $\mathrm{S}=0$ state instead of usual spin state of $\mathrm{S}=1$ as for example observed for $\mathrm{Ni}^{2+}$ in $\mathrm{NiO}$ [28]. This also can be explained by the $3 \mathrm{~d}$ levels split due to the $\mathrm{D}_{4 \mathrm{~h}}$ symmetry environment, as shown in Fig.1.5. If $\mathrm{Ni}^{2+}$ is in the $\mathrm{S}=0$ spin state, then $\mathrm{NiPc}$ should be a diamagnetic material as demonstrated later in this dissertation.

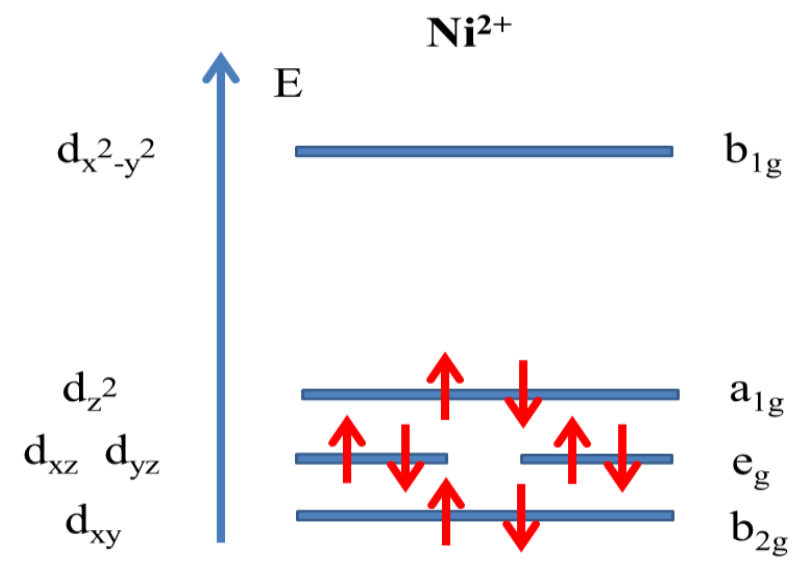

Fig. 1.5 Electron occupation of $3 d$ levels for the ground state of $\mathrm{Ni}^{2+}$ in NiPc [21]. 
For $\beta-\mathrm{CoPc}, \mathrm{Co}^{2+}\left(3 \mathrm{~d}^{7}\right)$ has been shown to be in spin $\mathrm{S}=1 / 2$ state rather than the usual high spin state $\mathrm{S}=3 / 2$ as for example in $\mathrm{CoO}$ [29]. Fig. 1.6 shows the electron occupation of $3 \mathrm{~d}$ levels for the ground state of $\mathrm{Co}^{2+}$ in $\beta$-CoPc. The difference between the situation of transition metals in TMPc systems and those in the oxides such $\mathrm{MnO}, \mathrm{CoO}$ and $\mathrm{NiO}[27-29]$ is that the crystal field in the latter systems has the cubic symmetry compared to the planar symmetry is the TMPc systems as evident in Fig. 1.3. In both $\alpha-\mathrm{CuPc}$ and $\beta-\mathrm{CuPc}, \mathrm{Cu}^{2+}$ is in the spin $\mathrm{S}=1 / 2$ state since there are nine electrons in the $3 \mathrm{~d}$ shell and one of these electrons has to fill out the $\mathrm{d}_{\mathrm{x}-\mathrm{y}}^{2}{ }^{2}$ level, as shown in Fig. 1.7.

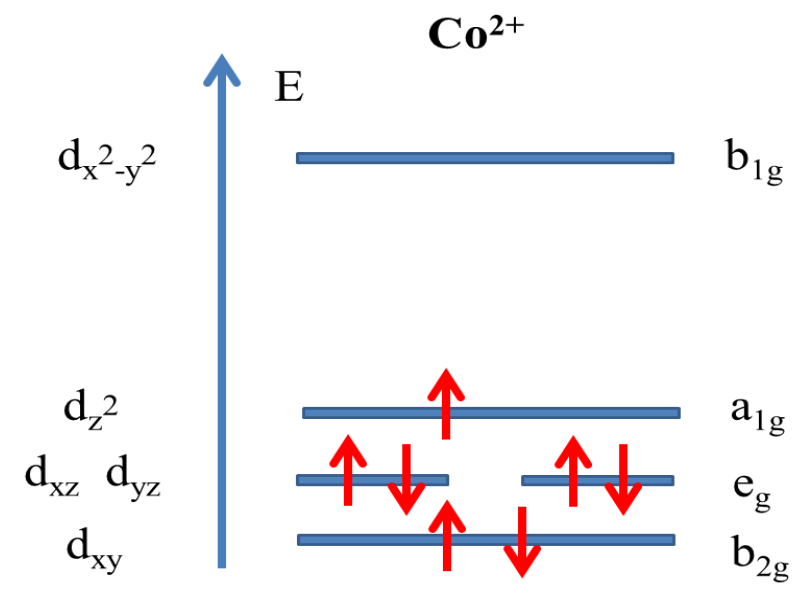

Fig. 1.6 Electron occupation of 3 d levels for the ground state of $\mathrm{Co}^{2+}$ in $\beta$-CoPc [21]. 


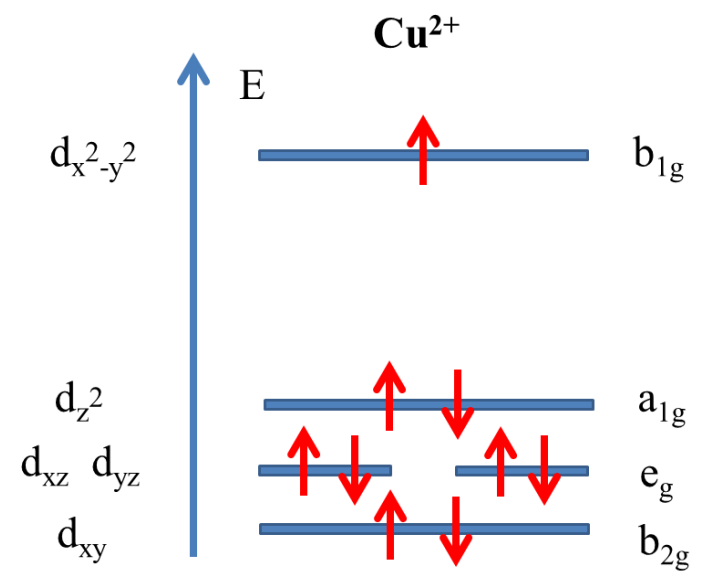

Fig. 1.7 Electron occupation of $3 d$ levels for the ground state of $\mathrm{Cu}^{2+}$ in $\alpha-\mathrm{CuPc}$ and $\beta-\mathrm{CuPc}[21]$.

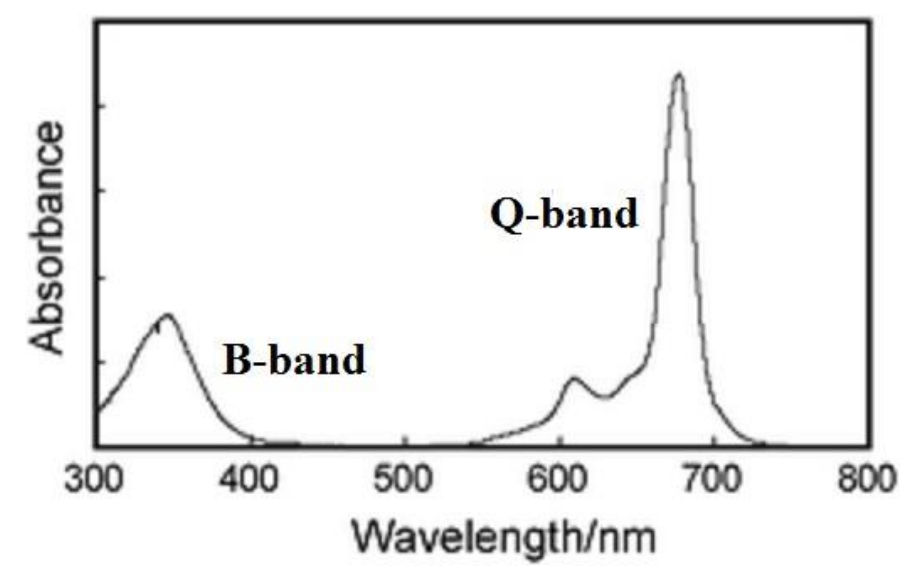

Fig. 1.8 Optical absorption spectrum of $\beta$-CuPc (adapted from [29]).

\subsection{Optical and Electrical Properties of TMPc}

The optical absorption spectrum of TMPc makes them excellent materials for pigments. For TMPc, there are two strong absorption bands denoted as Q-band and B-band, respectively (Fig. 1.8) [30]. Q-band is a strong absorption band in the visible region between $670 \mathrm{~nm}$ and $690 \mathrm{~nm}$ due to a $\pi$ - $\pi^{*}$ transition from the highest occupied molecular orbital (HOMO) to the lowest unoccupied molecular 
orbital (LUMO), and this is responsible for the characteristic intense blue (or blue-green) color of the TMPc compound. B-band is a strong absorption band in the ultra-violet (UV) region between $320 \mathrm{~nm}$ and $370 \mathrm{~nm}$. Also, TMPcs show remarkable nonlinear optical properties such as the intensity-dependent refractive index and the greater absorption coefficient of the excited state than ground state, which lead to potential applications in nonlinear optical devices [31-33].

Table 1.3 Electrical conductivity of TMPc thin films at room temperature.

\begin{tabular}{|l|l|l|}
\hline & $\sigma(\mathrm{S} / \mathrm{m})$ & Reference \\
\hline $\mathrm{CuPc}$ & $1.00 \times 10^{-4}$ & {$[34]$} \\
\hline $\mathrm{MnPc}$ & $1.01 \times 10^{-4}$ & {$[35]$} \\
\hline $\mathrm{CoPc}$ & $9.17 \times 10^{-4}$ & {$[36]$} \\
\hline $\mathrm{FePc}$ & $4.54 \times 10^{-4}$ & {$[37]$} \\
\hline $\mathrm{NiPc}$ & $2.75 \times 10^{-4}$ & {$[38]$} \\
\hline
\end{tabular}

Semiconducting behavior has been observed in TMPc materials (Table 1.3). As a result, their electrical properties of TMPc are becoming a fast-growing research area in order to make organic nano-electronic devices. Vacuum thermal evaporation, organic molecular beam epitaxy (OMBE), Langmuir-Blogett (LB) method, and spin-coating are the main methods to fabricate TMPc thin films [39]. High quality of thin films, good conductivity, and high thermal stability are the key advantages of TMPc 
offered to the organic nano-electronics community. One of the most famous examples is the p-type semiconductor $\mathrm{CuPc}$ for which carrier mobility reaches up to $10^{-2} \mathrm{~cm}^{2} / \mathrm{Vs}$. So CuPc has been widely used as the hole transport layer in OLED and OSC [40, 41]. TMPc also have been of considerable interest for potential applications in transistors, gas sensors, and memory devices [42].

\subsection{Magnetic properties of TMPc systems}

As discussed in section 1.2 , TMPc are potential $1 \mathrm{D}$ material magnetically because the direct and/or superexchange interaction between the nearest neighboring TM atoms along the monoclinic b-axis.

In 1960s, Lever performed magnetization vs. temperature measurements for several TMPc, fit the experimental data to Curie-Weiss law getting some information on exchange coupling [43]. However these measurements were quite limited in that the temperature range covered was only from $100 \mathrm{~K}$ to about $325 \mathrm{~K}$. The main conclusions of the paper were that TMPc may have ferromagnetic (FM) or antiferromagnetic coupling (AFM) between the TM ions with very different exchange constants $\left(\mathrm{J}_{\mathrm{ex}} / \mathrm{k}_{\mathrm{B}}\right)$ although they share very similar crystal structure. Since then, a number of papers have been published on magnetic properties of TMPc in the following decades. The nature of magnetism in $\mathrm{CuPc}$ [9] and $\beta$-CoPc $[22,23]$ have been reported to be Heisenberg linear chain antiferromagnets (HLCA) with spin $\mathrm{S}=1 / 2$ but without 3-dimensional (3D) long range order following the predictions of the Bonner-Fisher (BF) model [44]. However, the peak in the magnetization vs. temperature predicted by the BF model was not observed due to the limited temperature range of measurements. On the other hand, $\beta$-MnPc has been reported to be a canted ferromagnet below $\mathrm{T}_{\mathrm{C}} \approx 8.6 \mathrm{~K}$. [14, 45-47]. However, the recent result of the lack of a $\lambda$-type anomaly in the specific heat vs. temperature data near the quoted $\mathrm{T}_{\mathrm{C}}$ has questioned the 
presence of long-range 3D magnetic ordering in this system $[19,48]$. NiPc has been reported to be a diamagnetic material but without detailed experimental data [43]. Overall, the reported results for TMPc have been primarily based on DC magnetization measurement in a limited temperature range.

\subsection{Objectives of this Research and Outline of the Procedures employed}

In order to better understand the nature of magnetism in the TMPc systems, more detailed magnetic measurements covering the wider temperature range of $0.4 \mathrm{~K}$ to $350 \mathrm{~K}$ and in high magnetic fields up to $90 \mathrm{kOe}$ were undertaken. The objective of these investigations was the accurate determination of the nature of magnetism and exchange constants in the TMPc systems with TM = VO, Mn, Co, Ni and $\mathrm{Cu}$. These investigations include not only the temperature and magnetic field dependence of DC magnetization but also those of the AC magnetic susceptibilities $\chi^{\prime}$ and $\chi^{\prime \prime}$ in the frequency range of $0.1 \mathrm{~Hz}$ to $10 \mathrm{kHz}$. To achieve these objectives, the use of not only the in-house Physical Properties Measurements System (PPMS) was employed but also the facilities available at the National High Magnetic Field Laboratory (NHFML) at Florida State University in Tallahassee, FL and those available at the High Magnetic Field Laboratory, Chinese Academy of Sciences (CHMFL) in China were utilized. The majority of the magnetic measurements covering the temperature from $2 \mathrm{~K}$ to $350 \mathrm{~K}$ and magnetic field up to 90 kOe were carried out at West Virginia University using the PPMS manufactured by Quantum Design Inc. Additional magnetic measurements for $\beta-\mathrm{CoPc}$ at the lower temperatures of $0.4 \mathrm{~K}$ to $20 \mathrm{~K}$ were performed using a SCM2 magnet system at the National High Magnetic Field Laboratory (NHMFL) in Florida. For VOPc, a Magnetic Property Measurement System (MPMS) manufactured by Quantum Design Inc. and available at the High Magnetic Field Laboratory, Chinese Academy of Sciences in China 
was accessed to acquire data in the low temperature range of $0.5 \mathrm{~K}$ to $2 \mathrm{~K}$.

For interpretation of the data in $\alpha-\mathrm{CuPc}, \beta-\mathrm{CuPc}$ and $\beta-\mathrm{CoPc}$, magnetization vs. temperature data were fitted to the $\mathrm{BF}$ model offering more accurate results of the exchange constant $\mathrm{J}_{\mathrm{ex}} / \mathrm{k}_{\mathrm{B}}$. For $\beta-\mathrm{MnPc}$, both $\mathrm{DC}$ and $\mathrm{AC}$ magnetization data, and specific heat data lead to the conclusion that the 3D long-range magnetic ordering is absent in this material contrary to the conclusions reached in all previous investigations on this system. For $\beta$-NiPc, temperature dependence of magnetization and isothermal data at various temperatures confirmed it to be a diamagnetic material. For VOPc, temperature dependence of magnetization and isothermal data at various temperatures from $0.5 \mathrm{~K}$ to $300 \mathrm{~K}$ shows the very weak antiferromagnetic exchange coupling $(\theta=-0.1 \mathrm{~K})$ between $\mathrm{VO}^{2+}$ ions which implies the near-perfect paramagnetism in VOPc. Details of these new results, analysis and their discussion are presented in the following chapters.

\subsection{Organization of the Dissertation}

Chapter 2 outlines the theory of magnetism relevant to TMPc systems including Brillouin function, Curie-Weiss law, effects of crystal field, spin-orbit interaction, Heisenberg exchange interaction, Bonner-Fisher (BF) model, and Ising chain model. In Chapter 3, brief descriptions of the experimental techniques and procedures employed in these investigations are given. In Chapter 4, experimental results and their analysis on the temperature and magnetic field dependence of the magnetization in powder samples of $\alpha$ - and $\beta$-CuPc followed by the discussion of the electronic state of $\mathrm{Cu}$ and the exchange interactions are presented. Chapter 5 deals with similar data and analysis for the powder sample of $\beta$-CoPc. In this sample, a peak in the $\chi$ vs. T data predicted by the BF model was observed for the first 
time and the exchange constant $\mathrm{J}_{\mathrm{ex}} / \mathrm{k}_{\mathrm{B}}$ was accurately determined by fitting $\chi \mathrm{vs}$. T data to the $\mathrm{BF}$ model over the wide temperature range of $0.4 \mathrm{~K}$ to $300 \mathrm{~K}$. In Chapter 6 , magnetic properties of the powder sample of VOPc covering the temperature range of $0.5 \mathrm{~K}$ to $300 \mathrm{~K}$ and in magnetic fields up to $90 \mathrm{kOe}$ are presented, analyzed and discussed. The magnetization data fits well with the Curie-Weiss law with very small $\theta=-0.1 \mathrm{~K}$ which implies the near-perfect paramagnetic nature of VOPc. In Chapter 7, temperature and magnetic field dependence of the magnetization of powder sample of $\beta$-NiPc is presented confirming the overall diamagnetism in this material. In Chapter 8 , detailed measurements of both the temperature and magnetic field dependence of the $\mathrm{DC}$ and $\mathrm{AC}$ magnetic susceptibilities of powder sample of $\beta$-MnPc are presented followed by thorough discussion and analysis. From this analysis, the exchange constant $\mathrm{J}_{\mathrm{ex}} / \mathrm{k}_{\mathrm{B}}$ and the single ion anisotropy are determined. In addition, a Cole-Cole analysis is carried out by plotting the ac susceptibilities $\chi^{\prime \prime}$ vs. $\chi^{\prime}$ at different temperatures to determine distribution in the magnetic relaxation rate and it is concluded that this system lacks 3-dimensional long range magnetic ordering contrary to conclusions reached in previous publications. In Chapter 9, a summary of the major results resulting from this work is presented followed by suggestions for future studies. A list of publications and presentations by the author are also included in this chapter. Finally, in appendix A, a copy of the proposal submitted to the National High Magnetic Field Laboratory for low temperature magnetic measurements of $\beta$-CoPc is presented and in appendix $\mathrm{B}$, typical computer sequences used for measuring the magnetic properties using the PPMS are listed.

\section{References}

[1] V.A. Dediu, L.E. Hueso, L. Bergenti and C. Taliani, Nat. Mater. 8, 707 (2009). 
[2] S.R. Forrest, Nat. 428, 911 (2004).

[3] M. Berggren and A. Richter-Dahlfors, Adv. Mater. 19, 3201 (2007).

[4] R.P. Linstead and A.R. Lowe, J. Chem. Soc. 1022 (1934).

[5] R.P. Linstead and C.E. Dent, J. Chem. Soc. 1027 (1934).

[6] J.M. Robertson, J. Chem. Soc. 615 (1935).

[7] J.M. Robertson, J. Chem. Soc. 1195 (1935).

[8] S.M. Tadayyon, H.M. Grandin, K. Griffiths, P.R. Norton, H. Azizi and Z.D. Popovic, Org. Electron. 5, 157 (2004).

[9]. H. Wang, S. Mauthoor, S. Din, J. A. Gardener, R. Chang, M. Warner et al. ACS Nano. 4, 3921, (2010).

[10]. M. Warner, S. Din, I. S. Tupitsyn, G. W. Morley, A. M. Stoneham, J. A. Gardener et al. Nature. 503, 505 (2013).

[11] P. Ballirano, R. Caminiti, C. Ercolani, A. Maras and M.A. Orru, J. Am. Chem. Soc. 120, 12798 (1998).

[12] J.M. Assour, J. Phys. Chem. 69 (7), 2295 (1965).

[13] C. Ercolani, C. Neri and P. Porta, Inorg. Chim. Acta. 1, 415 (1967).

[14] C.G. Barraclough, R.L. Martin, S. Mitra and R.C. Sherwood, J. Chem. Phys. 53, 1638 (1970). 
[15] P. W. Anderson, Phys. Rev. 115, 2 (1959).

[16] In the space groups $\mathrm{C} 2 / \mathrm{c}$ and $\mathrm{P} 2{ }_{1} / \mathrm{c}$ of the monoclinic structure in Table 1.1, the first letter represents the Bravais lattice: $\mathrm{P}$ for primitive and $\mathrm{C}$ for centered. Next, the number 2 represents the presence of a two-fold $\left(180^{\circ}\right)$ rotation axis which for monoclinic systems is the b-axis and $2_{1}$ represents a two-fold rotation axis followed by a translation of $1 / 2$ of the lattice vector along the rotation axis. Finally /c represents a c-glide plane perpendicular to the b-axis.

[17] Z. Wang, K.L. Pisane and M.S. Seehra, IEEE. Trans. Mag. 51, 2700104 (2015).

[18] C. Kittel, Introduction to Solid State Physics, $8^{\text {th }}$ edition. Wiley, 2004.

[19] Y. Taguchi, T. Miyake, S. Margadonna, K. Kato and Y. Iwasa, J. Am. Chem. Soc. 128, 3313 (2006).

[20] T. Kataoka, Y. Sakamoto, Y. Yamazaki, V.R. Singh, A. Fujimori, Y. Takeda, T. Ohkochi, S.-I. Fujimori, T. Okane, Y. Saitoh, H. Yamagami and A. Tanaka, Solid State Communs. 152, 806 (2012).

[21] A. Mugarza, R. Robles, C. Krull, R. Korytar, N. Lorente and P. Gambardella, Phys. Rev. B. 85, 155437 (2012).

[22] M. Serri, W.Wu, L.R. Fleet, N.M. Harrison, C.F. Hirjibehedin, C.W.M. Kay, A.J. Fisher, G. Aeppli and S. Heutz, Nature Commun. 5, 3079 (2014).

[23] W. Wu, N.M. Harrison and A.J. Fisher, Phys. Rev. B. 88, 024436 (2013).

[24] J.S. Griffith and L.E. Orgel, Rev. Chem. Soc. 11, 381 (1957). 
[25] 3d electron orbital: http://socratic.org/

[26] I.E. Brumboiu, R. Totani, M.D. Simone, M. Coreno, C. Grazioli, L. Luca, H.C. Herper, B. Sanyal, O.

Eriksson, C. Puglia and B. Brena, J. Phys. Chem. A. 118, 927 (2014).

[27] M. S. Jagadeesh and M. S. Seehra, Phys. Rev. B. 21, 2897 (1980).

[28] G. Srinivasan and M. S. Seehra, Phys. Rev. B. 29, 6295 (1984).

[29] P. Silinsky and M. S. Seehra, Phys. Rev. B. 24, 419 (1981).

[30] H. Yaku, T. Fujimoto, T. Murashima, D. Miyoshi and N. Sugimoto, Chem. Commu. 48, 6203 (2012).

[31] J.S. Shirk, J.R. Lindle, F.J. Bartoli, C.A. Hoffman, Z.H. Kafafi and A.W. Snow, Appl. Phys. Lett. 55, 1287 (1989).

[32] G. de la Torre, P. Vazquez, F. Agullo-Lopez and T. Torres, J. Mater. Chem. 8, 1671 (1998).

[33] G. de la Torre, P. Vazquez, F. Agullo-Lopez and T. Torres, Chem. Rev. 104, 3723 (2004).

[34] A. Sussman, J. Appl. Phys. 38, 2748 (1967).

[35] K.R. Rajesh and C.S. Menon, Eur. Phys. J. B. 47, 171 (2005).

[36] S. Ambily and C.S. Menon, Indian J. Pure Appl. Phys. 37, 566 (1999).

[37] K.P. Khrishnakumar and C.S. Menon, Mater. Lett. 48, 64 (2001).

[38] K.N. Narayanan Unni and C.S. Menon, Mater. Lett. 45, 326 (2000). 
[39] M. Ling and Z. Bao, Chem. Mater. 16, 4824 (2004).

[40] C.W. Tang, Appl. Phys. Lett. 48, 183 (1986).

[41] S.M. Tadayyon, H.M. Grandin, K. Griffiths, P.R. Norton, H. Aziz and Z.D. Popovic, Org. Electron. 5, 157 (2004).

[42] N.B. McKeown, Phthalocyanine Materials: Synthesis, Structure and Function. Cambridge, U.K.: Cambridge Univ. Press, 1998.

[43] A.B.P. Lever, J. Chem. Soc. 1821 (1965).

[44] J.C. Bonner and M.E. Fisher, Phys. Rev. 135, A640 (1964).

[45] C.G. Barraclough, A.K. Gregson and S. Mitra, J. Chem. Phys. 60, 962 (1974).

[46] H. Miyoshi, H. Ohya-Nishiguchi and Y. Deguchi, Bull. Chem. Soc. Jpn. 46, 2724 (1974).

[47] H. Miyoshi, Bull. Chem. Soc. Jpn. 47, 561 (1974).

[48] Y. Taguchi, T. Miyake, S. Margadonna, K. Kato, K. Prassides and Y. Iwasa, J. Magn. Magn. Mater. 310, 1229 (2007). 


\section{CHAPTER 2.}

\section{Magnetism: Background and Theoretical Considerations}

\subsection{Background}

The history of magnetism dates back to about $600 \mathrm{BC}$ when ancient Greeks first used a mineral called lodestone which is now known to contain ferrimagnetic magnetite $\left(\mathrm{Fe}_{3} \mathrm{O}_{4}\right)$. The lodestone was called a magnet because of its ability to attract other pieces of the same material and iron. However, the nature of magnetism was first truly studied by Englishman William Gilbert (1540-1603) who investigated the phenomenon of magnetism systematically using scientific methods and published his classic book $O n$ the Magnet in 1600. Gilbert also discovered that the earth itself is a magnet and German Carl Friedrich Gauss (1777-1855) carried out the early theoretical investigations into the nature of the earth's magnetism. Frenchman Charles Coulomb (1736-1806) initiated the quantitative studies of magnetic phenomena in $18^{\text {th }}$ century, established the inverse square law of the magnetic force. The link between electricity and magnetism was first suggested by Danish physicist Hans Christian Oersted (1777-1851), later was proved by Frenchman Andre Marie Ampere (1775-1836) and Englishman Michael Faraday (1791-1869), who conducted experiments.

The modern understanding of magnetic phenomena was initiated by Pierre Curie (1859-1906) and his wife Madame Marie Curie (1867-1934), Paul Langevin (1872-1946), and Pierre Weiss (1865-1940). In 1895, Pierre Curie first reported the measurements of the magnetization of a large number of substances over an extended range of temperature and proposed the Curie law. Later (in 1905) 
Paul Langevin proposed theories on both diamagnetism and paramagnetism without taking into account of interaction between the individual carriers of magnetic moment (atoms or molecules) [1]. In 1907 Weiss pointed out that the paramagnetism can be understood by postulating the elementary moments do interact with one another through so called 'molecular field' and his theory lead to the Curie-Weiss law [2]. With the powerful tool of quantum mechanics, especially the spin magnetic moment, the modern theory on magnetism was finally established in the first half of $20^{\text {th }}$ century. The material given below in Sections 2.2, 2.3, 2.4 and 2.5 using the quantum theory of magnetism is based on the books by Moorish [3] and Pake [4]. These concepts and equations are used in subsequent chapters for the interpretation of the experimental data.

\subsection{Magnetic Moment, Brillouin Function, and Curie-Weiss Law}

In quantum theory, the magnetic moment of an atom or ion in free space is given by [3]:

$$
\vec{\mu}=-g \mu_{\mathrm{B}} \overrightarrow{\mathrm{J}}
$$

where the total angular momentum $\vec{J}$ is the vector sum of orbital angular momentum $\vec{L}$ and spin angular momentum $\vec{S} \cdot \mu_{\mathrm{B}}=\mathrm{eh} / 4 \pi \mathrm{mc}=9.274 \times 10^{-21} \mathrm{emu}$ is called the Bohr Magneton and the negative sign of the electron charge is taken into account in writing Eq. (2.1). In Eq. (2.1), g is called the Lande g-factor with $g=1$ and $g=2$ are for the orbital and spin angular momentum respectively. For a free atom, the Lande g-factor is given by: 


$$
g=1+\frac{J(J+1)-S(S+1)-L(L+1)}{2 J(J+1)}
$$

In an applied magnetic field, the Hamiltonian and potential energy of a magnetic moment

are given by

$$
\begin{gathered}
\mathcal{x}=-\vec{\mu} \cdot \overrightarrow{\mathrm{H}}=\mathrm{g} \mu_{\mathrm{B}} \overrightarrow{\mathrm{J}} \cdot \overrightarrow{\mathrm{H}}=\mathrm{g} \mu_{\mathrm{B}} \mathrm{HJ} \\
\mathrm{E}=\mathrm{g} \mu_{\mathrm{B}} \mathrm{Hm}_{\mathrm{J}}, \mathrm{m}_{\mathrm{J}}=-\mathrm{J},-\mathrm{J}+1, \ldots, \mathrm{J}-1, \mathrm{~J}
\end{gathered}
$$

where $m_{J}$ is called the azimuthal quantum number. For example: for $J=1 / 2, m_{J}=1 / 2,-1 / 2$ giving two levels with energy $\mathrm{E}=-\mathrm{g} \mu_{\mathrm{B}} \mathrm{H} / 2$ and $\mathrm{g} \mu_{\mathrm{B}} \mathrm{H} / 2$. For $\mathrm{J}=1, \mathrm{~m}_{\mathrm{J}}=1,0$, -1 leading to three levels with energy $\mathrm{E}$ $=g \mu_{\mathrm{B}} \mathrm{H}, 0,-\mathrm{g} \mu_{\mathrm{B}} \mathrm{H}$. Once the energy levels are known, the magnetization $\mathrm{M}$ defined as the magnetic moment per unit volume with $\mathrm{N}$ being the number of atoms or molecules per unit volume, can be determined from the equation [3]:

$$
M=-N \frac{\sum_{n} \frac{\partial E_{n}}{\partial H} e^{-\frac{E_{n}}{k_{B} T}}}{\sum_{n} e^{-\frac{E_{n}}{k_{B} T}}}
$$

Using $\quad \mathrm{E}=\mathrm{g} \mu_{\mathrm{B}} \mathrm{Hm}_{\mathrm{J}}$ from Eq. (2.4), it can be shown that Eq. (2.5) reduces to

$$
\mathrm{M}=\mathrm{Ng} \mu_{\mathrm{B}} J \mathrm{~B}_{\mathrm{J}}(\mathrm{y})=\mathrm{M}_{\mathrm{S}} \mathrm{B}_{\mathrm{J}}(\mathrm{y})
$$

where $\mathrm{y}=\mathrm{g} \mu_{\mathrm{B}} \mathrm{HJ} /\left(\mathrm{k}_{\mathrm{B}} \mathrm{T}\right), \mathrm{M}_{\mathrm{S}}=\mathrm{Ng} \mu_{\mathrm{B}} \mathrm{J}$ is the saturation magnetization, and $\mathrm{B}_{\mathrm{J}}(\mathrm{y})$ is given by 


$$
\mathrm{B}_{\mathrm{J}}(\mathrm{y})=\frac{2 \mathrm{~J}+1}{2 \mathrm{~J}} \operatorname{coth}\left(\frac{2 \mathrm{~J}+1}{2 \mathrm{~J}} \mathrm{y}\right)-\frac{1}{2 \mathrm{~J}} \operatorname{coth}\left(\frac{\mathrm{y}}{2 \mathrm{~J}}\right)
$$

$\mathrm{B}_{\mathrm{J}}(\mathrm{y})$ is called the Brillouin function (shown as Fig. 2.1) and was first obtained in 1927. The importance of Eq.(2.5) is that it allows calculation of $\mathrm{M}$ for any system once the energy level of the system are known under the action of an applied magnetic field $\mathrm{H}$.

For the special case of $J=1 / 2, m_{J}=1 / 2,-1 / 2$, the energy levels are $E_{n}=g \mu_{B} H / 2$ and $g \mu_{B} H / 2$. Substituting these in Eq. (2.5) with $x=\frac{g \mu_{B} H}{2 k_{B} T}$ and $M_{S}=\frac{1}{2} N g \mu_{B}$ yields

$$
\begin{aligned}
M & =\frac{1}{2} \operatorname{Ng} \mu_{B} \frac{e^{x}-e^{-x}}{e^{x}+e^{-x}} \\
& =M_{S} \tanh \left(\frac{g \mu_{B} H}{2 k_{B} T}\right)
\end{aligned}
$$

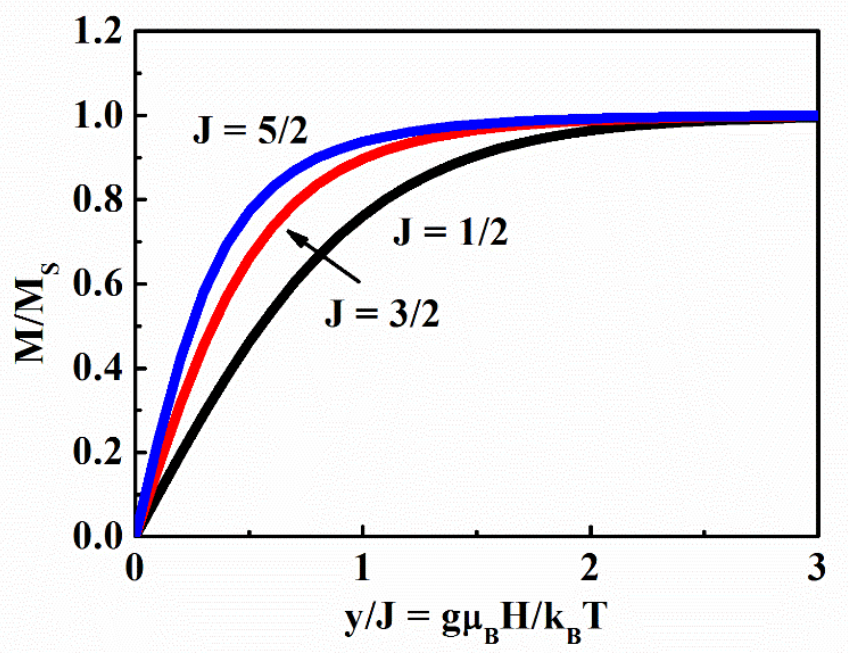

Fig. 2.1 Plot of Brillouin function with $J=1 / 2,3 / 2$, and 5/2.

For $\mathrm{y}<<1$ (low field and high temperature case), it can be shown that Eq. (2.6) yields 


$$
M=\frac{N g^{2} \mu_{B}^{2} J(J+1)}{3 k_{B} T} H
$$

From Eq. (2.9), the magnetic susceptibility is given by

$$
\chi=\frac{\partial \mathrm{M}}{\partial \mathrm{H}}=\frac{\mathrm{N} \mu^{2}}{3 \mathrm{k}_{\mathrm{B}} \mathrm{T}}=\frac{\mathrm{C}}{\mathrm{T}}
$$

where $\mu=g \mu_{B} \sqrt{J(J+1)}$ is called the effective magnetic moment and $\mathrm{C}=\mathrm{N} \mu^{2} /\left(3 \mathrm{k}_{\mathrm{B}}\right)$ is known as the Curie constant. Eq. (2.10) is called Curie law (1/T variation of magnetic susceptibility). For the classical case when $\mathrm{J}=\infty$, the Brillouin function reduces to the Langevin function L(y) (classical theory) given by

$$
\mathrm{L}(\mathrm{y})=\operatorname{coth}(\mathrm{y})-\frac{1}{y}
$$

So the magnetization becomes

$$
\mathrm{M}=\mathrm{M}_{\mathrm{S}} \mathrm{L}(\mathrm{y})=\mathrm{M}_{\mathrm{S}}\left[\operatorname{coth}(\mathrm{y})-\frac{1}{\mathrm{y}}\right]
$$

If $\mathrm{y}=\mathrm{g} \mu_{\mathrm{B}} \mathrm{HJ} /\left(\mathrm{k}_{\mathrm{B}} \mathrm{T}\right) \ll<1$ (low field and high temperature case), the Langevin function can be expressed as a series, it is

$$
\mathrm{L}(\mathrm{y})=\frac{\mathrm{y}}{3}-\frac{\mathrm{y}^{3}}{45}+\frac{2 \mathrm{y}^{5}}{945}-\cdots \approx \frac{y}{3}
$$

$\mathrm{L}(\mathrm{y})$ as a function of $\mathrm{y}$ is plotted in Fig. 2.2. The solid line is the Langevin function whereas the dash line represents the Langevin function for $\mathrm{y}<<1$. 


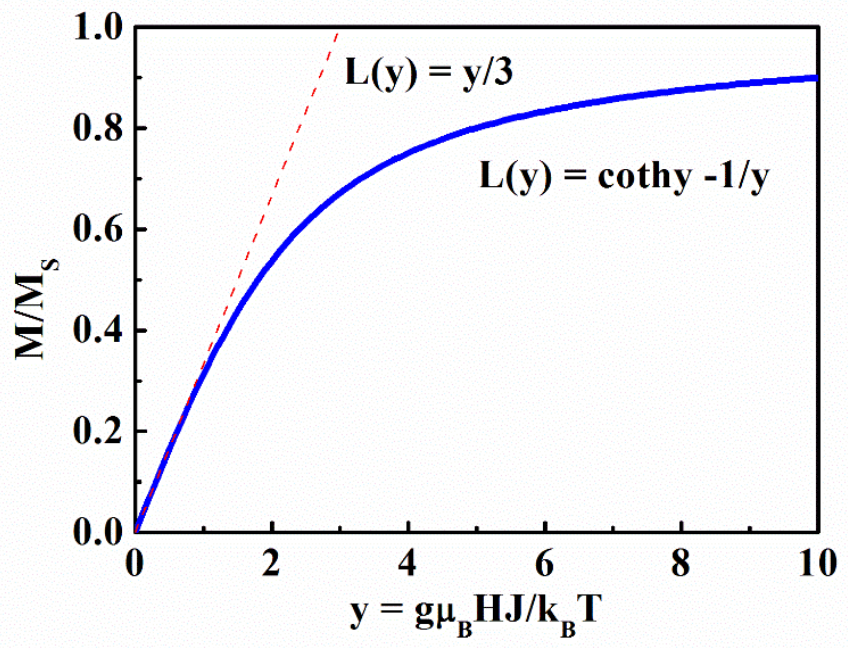

Fig. 2.2 Variation of normalized magnetization with normalized magnetic field, Eq. (2.12)

The above derivation of Curie law assumes no interaction between magnetic moments. In 1907,

Weiss proposed the presence of an effective field $\mathrm{H}_{M}$ due to interaction between magnetic moments proportional to the magnetization given by:

$$
\mathrm{H}_{\mathrm{M}}=\mathrm{N}_{\mathrm{W}} \mathrm{M}
$$

where $\mathrm{N}_{\mathrm{W}}$ is the molecular field constant. Then the total field is

$$
\mathrm{H}_{\mathrm{T}}=\mathrm{H}+\mathrm{H}_{\mathrm{M}}
$$

For Brillouin function $\mathrm{M}=\mathrm{Ng} \mu_{\mathrm{B}} \mathrm{JB}_{\mathrm{J}}(\mathrm{y})$ with $\mathrm{y}=\mathrm{g} \mu_{\mathrm{B}} \mathrm{JH} / \mathrm{k}_{\mathrm{B}} \mathrm{T}, \quad \mathrm{H}$ is replaced by $\mathrm{H}_{\mathrm{T}}=\mathrm{H}+\mathrm{H}_{\mathrm{M}}$ 


$$
y=\frac{g \mu_{B} J}{k_{B} T} H_{T}=\frac{g \mu_{B} J}{k_{B} T}\left(H+H_{M}\right)=\frac{g \mu_{B} J}{k_{B} T}\left(H+N_{W} M\right)
$$

For $\mathrm{y}<<1, \mathrm{~B}_{\mathrm{J}}(\mathrm{y})=\frac{(\mathrm{J}+1)}{3 \mathrm{~J}} \mathrm{y}$

$$
M=N g \mu_{B} J \frac{(J+1)}{3 J} y=\frac{N g \mu_{B}(J+1)}{3} \cdot \frac{g \mu_{B} J\left(H+N_{W} M\right)}{k_{B} T}=\frac{N g^{2} \mu_{B}^{2} J(J+1)}{3 k_{B} T}\left(H+N_{W} M\right)
$$

with $\mathrm{C}=\mathrm{N} \mu^{2} /\left(3 \mathrm{k}_{\mathrm{B}}\right)=\mathrm{Ng}^{2} \mu_{\mathrm{B}}^{2} \mathrm{~J}(\mathrm{~J}+1) / 3 \mathrm{k}_{\mathrm{B}}$, then

$$
\begin{gathered}
\mathrm{M}=\frac{\mathrm{C}}{\mathrm{T}}\left(\mathrm{H}+\mathrm{N}_{\mathrm{W}} \mathrm{M}\right) \\
\mathrm{M}=\frac{\mathrm{CH}}{\mathrm{T}-\mathrm{CN}_{\mathrm{W}}}
\end{gathered}
$$

The magnetic susceptibility is then given by

$$
\chi=\frac{\partial M}{\partial H}=\frac{C}{T-C N_{W}}=\frac{C}{T-\theta}
$$

where the Weiss constant $\theta=C \cdot N_{W}=T_{C}$ and the Curie constant $C=N \mu^{2} / 3 k_{B}$. Eq. (2.19) is the Curie-Weiss (CW) law. If $\theta>0$, the material is a ferromagnet and the ordering temperature $\mathrm{T}_{\mathrm{C}}=\theta$ is called the Curie temperature. If $\theta<0$, the material is an antiferromagnet, the ordering temperature $\mathrm{T}_{\mathrm{N}}=\theta$ is called the Neel temperature.

\subsection{Effect of Crystal Field}

In a solid, the energy level of an electron on a site is affected by the crystal field of the neighboring ions [4]. Consider a $3 \mathrm{~d}^{1}$ electron (such as in VOPc discussed later in this dissertation) in a 
cubic field of six negative ions at a distance of $a_{0}$. The wave functions of the electron must obey the symmetry of the crystalline field. These orbitals of the electron, constructed from spherical harmonics of $l$ $=2$, are shown in Chapter 1 (Fig 1.2). It is straightforward to show that for these orbitals, the expectation value of $\langle\mathrm{Lz}\rangle$ is zero i.e. the orbital momentum is quenched. The five-fold degeneracy of $l=2$ state is lifted by the crystal field due to Stark splitting, leading to a triplet (xy, yz, zx orbital) and a doublet ( $\mathrm{z}^{2}$, $\mathrm{x}^{2}-\mathrm{y}^{2}$ orbital) as shown in Fig 2.3. If the crystal filed is tetragonal, then there is a further splitting of the energy levels as shown in Fig 2.3. Depending on the strength of the crystal field, the magnitudes of the parameters $\Delta \approx 10^{4} \mathrm{~cm}^{-1}$ and $\delta \approx 10^{2}$ to $10^{3} \mathrm{~cm}^{-1}$. So at low temperatures, only the lowest energy levels will be occupied. Optical absorption measurements are usually used to determine the magnitudes of $\Delta$ and $\delta$. The spin of the electron doubles the degeneracy of each level with $+(-)$ designating the up (down) spin. In an applied magnetic field, this degeneracy is lifted due to the Zeeman splitting.

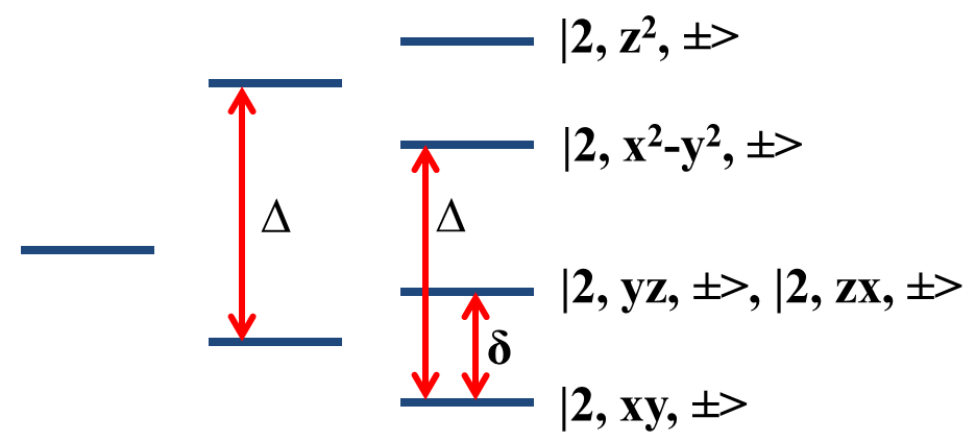

free cubic tetragonal ion field field

Fig. 2.3 Splitting of the $3 d^{l}$ electron energy level due to cubic field $(\Delta)$ and tetragonal field $(\delta)$. 


\subsection{Effect of Spin-Orbit Interaction}

As noted earlier, the expectation values of the angular momentum $\left\langle\mathrm{L}_{\mathrm{z}}\right\rangle$ can be shown to be zero for each of the Stark-split levels of Fig 2.3. The spin-orbit interaction $\lambda \overrightarrow{\mathrm{L}} \bullet \overrightarrow{\mathrm{S}}$ partially restores some of this orbit contribution to the magnetic moment by mixing some of the excited states into the ground state.

By writing $\lambda \overrightarrow{\mathrm{L}} \cdot \overrightarrow{\mathrm{S}}$ in terms of raising and lowering operators:

$$
\mathrm{H}^{\prime}=\lambda \overrightarrow{\mathrm{L}} \cdot \overrightarrow{\mathrm{S}}=\lambda\left[\mathrm{L}_{\mathrm{z}} \mathrm{S}_{\mathrm{z}}+\frac{1}{2}\left(\mathrm{~L}_{+} \mathrm{S}_{-}+\mathrm{L}_{-} \mathrm{S}_{+}\right)\right]
$$

Here $\lambda$ is the spin-orbit coupling constant, $\mathrm{L}_{ \pm}=\mathrm{L}_{\mathrm{x}} \pm \mathrm{i} \mathrm{L}_{\mathrm{y}}$, and $\mathrm{S}_{ \pm}=\mathrm{S}_{\mathrm{x}} \pm \mathrm{i} \mathrm{S}_{\mathrm{y}}$. Now consider the effect of Eq. (2.20) on the states shown in Fig. 2.3 using the first order perturbation theory according to which

$$
\begin{gathered}
\mathrm{H}=\mathrm{H}_{0}+\mathrm{H}^{\prime} \text { (perturbation) } \\
\text { with } \mathrm{H}_{0}\left|\mathrm{n}^{(0)}>=\mathrm{E}_{\mathrm{n}}^{0}\right| \mathrm{n}^{(0)}> \\
\text { and } \mathrm{H}\left|\mathrm{n}>=\mathrm{E}_{\mathrm{n}}\right| \mathrm{n}> \\
\mathrm{E}_{\mathrm{n}}=\mathrm{E}_{\mathrm{n}}^{(0)}+<\mathrm{n}^{(0)}\left|\mathrm{H}^{\prime}\right| \mathrm{n}^{(0)}>+\sum_{\mathrm{m} \neq \mathrm{n}} \frac{<\mathrm{n}^{(0)}\left|\mathrm{H}^{\prime}\right| \mathrm{m}^{(0)}><\mathrm{m}^{(0)}\left|\mathrm{H}^{\prime}\right| \mathrm{n}^{(0)}>}{\mathrm{E}_{\mathrm{n}}^{(0)}-\mathrm{E}_{\mathrm{m}}^{(0)}} \\
\text { and }|\mathrm{n}>=| \mathrm{n}^{(0)}>+\sum_{\mathrm{m} \neq \mathrm{n}} \frac{<\mathrm{m}^{(0)}\left|\mathrm{H}^{\prime}\right| \mathrm{n}^{(0)}>}{\mathrm{E}_{\mathrm{n}}^{(0)}-\mathrm{E}_{\mathrm{m}}^{(0)}} \mid \mathrm{m}^{(0)}>
\end{gathered}
$$

The total effect of the $\lambda \overrightarrow{\mathrm{L}} \bullet \overrightarrow{\mathrm{S}}$ term on the ground state $\mid 2, \mathrm{xy}, \pm>$ is to modify this state to 


$$
| \pm>=| 2, \mathrm{xy}, \pm> \pm \frac{\mathrm{i} \lambda}{\Delta}\left|2, \mathrm{x}^{2}-\mathrm{y}^{2}, \pm>\mp \frac{\lambda}{2 \delta}\right| 2, \mathrm{yz}, \mp>+\frac{\mathrm{i} \lambda}{2 \delta} \mid 2, \mathrm{zx}, \mp>
$$

For the applied field along $\mathrm{z}$ direction, the Zeeman energy is given by

$$
\mathcal{x}=-\vec{\mu} \cdot \vec{H}=\frac{\mu_{B}}{\hbar}\left(L_{z}+2 S_{z}\right) H_{0}
$$

For the state given by Eq. (2.26), the expectation values of magnetic moment is given by-

$$
\begin{gathered}
\mu=\frac{\mu_{\mathrm{B}}}{\hbar}\left(\mathrm{L}_{\mathrm{z}}+2 \mathrm{~S}_{\mathrm{z}}\right) \\
<+\left|\mathrm{L}_{\mathrm{z}}+2 \mathrm{~S}_{\mathrm{z}}\right|+>=\frac{\hbar}{2}\left(2-\frac{8 \lambda}{\Delta}\right) \\
<-\left|\mathrm{L}_{\mathrm{z}}+2 \mathrm{~S}_{\mathrm{z}}\right|->=\frac{\hbar}{2}\left(-2+\frac{8 \lambda}{\Delta}\right)
\end{gathered}
$$

Substituting these values in Eq. (2.27) yields

$$
\begin{gathered}
<+|\mathrm{E}|+>=\frac{1}{2}\left(2-\frac{8 \lambda}{\Delta}\right) \mathrm{H}_{0} \mu_{\mathrm{B}} \\
<-|\mathrm{E}|->=\frac{1}{2}\left(-2+\frac{8 \lambda}{\Delta}\right) \mathrm{H}_{0} \mu_{\mathrm{B}} \\
\Delta \mathrm{E}=<+|\mathrm{E}|+>-<-|\mathrm{E}|->=\left(2-\frac{8 \lambda}{\Delta}\right) \mu_{\mathrm{B}} \mathrm{H}_{0}=\mathrm{g}_{\mathrm{z}} \mu_{\mathrm{B}} \mathrm{H}_{0} \\
\mathrm{~g}_{\mathrm{z}}=\mathrm{g}_{\mathrm{e}}-\frac{8 \lambda}{\Delta}
\end{gathered}
$$


with $\mathrm{g}_{\mathrm{e}}=2.0023 \approx 2$ for the free electron. For the field applied along $\mathrm{x}$ direction (also equivalent to $\mathrm{y}$ direction for tetragonal symmetry), a similar calculation yields

$$
\begin{gathered}
\mathcal{x}=-\vec{\mu} \bullet \overrightarrow{\mathrm{H}}=\frac{\mu_{\mathrm{B}}}{\hbar}\left(\mathrm{L}_{\mathrm{x}}+2 \mathrm{~S}_{\mathrm{x}}\right) \mathrm{H}_{0} \\
\mathrm{~g}_{\mathrm{x}}=\mathrm{g}_{\mathrm{y}}=\mathrm{g}_{\mathrm{e}}-2 \frac{\lambda}{\delta}
\end{gathered}
$$

Here $\lambda$ is positive (negative) if the shell is less (more) than half-filled. So the g-shift $\left(g-g_{e}\right)$ is negative (positive) for ions with shell less (more) than half full with electrons. In the following chapters, examples are given showing negative g-shift for $\mathrm{VO}^{2+}$ in VOPc and positive $\mathrm{g}$-shifts for $\mathrm{Co}^{2+}$ in $\mathrm{CoPc}, \mathrm{Mn}^{2+}$ in $\mathrm{MnPc}$ and $\mathrm{Cu}^{2+}$ in $\mathrm{CuPc}$.

\subsection{The Heisenberg Exchange Interaction}

Consider two electrons, a and b, located in the neighboring sites 1 and 2, such that $\psi_{\mathrm{a}}(1)$ and $\psi_{\mathrm{a}}(2)$ are the wave functions of electron a at site 1 and 2 and $\psi_{\mathrm{b}}(1)$ and $\psi_{\mathrm{b}}(2)$ are the wave functions of electron $\mathrm{b}$ at site 1 and 2 respectively [3]. In addition to the above spatial part of wavefunction, we also need to consider the spin part of wavefunction of the electrons. According to Pauli's Exclusion Principle, the total wavefunction must be antisymmetric with respect to interchange of electrons at site 1 and 2 . The total wavefunction is:

$$
\psi_{\text {total }}=\psi_{\text {space }} \cdot \psi_{\text {spin }}
$$

The spin part of the wavefunction is shown in the following table. 
Table 2.1 Spin part of the wavefunction.

\begin{tabular}{|c|l|l|l|}
\hline Wave function & $\mathrm{S}$ & $\mathrm{S}_{\mathrm{Z}}$ & \\
\hline$\left.\frac{1}{\sqrt{2}}(|\uparrow \downarrow>-| \downarrow \uparrow\rangle\right)$ & 0 & 0 & Singlet: antisymmetric \\
\hline $\mid \uparrow \uparrow>$ & 1 & 1 & \\
\hline$\frac{1}{\sqrt{2}}(|\uparrow \downarrow\rangle+\mid \downarrow \uparrow>)$ & 1 & 0 & \multirow{2}{*}{ Triplet: symmetric } \\
\hline $\mid \downarrow \downarrow>$ & 1 & -1 & \\
\hline
\end{tabular}

The spatial part of the wavefunction:

$$
\begin{aligned}
& \psi_{\mathrm{S}}=\frac{1}{\sqrt{2}}\left[\psi_{\mathrm{a}}(1) \psi_{\mathrm{b}}(2)+\psi_{\mathrm{b}}(1) \psi_{\mathrm{a}}(2)\right] \\
& \psi_{\mathrm{A}}=\frac{1}{\sqrt{2}}\left[\psi_{\mathrm{a}}(1) \psi_{\mathrm{b}}(2)-\psi_{\mathrm{b}}(1) \psi_{\mathrm{a}}(2)\right]
\end{aligned}
$$

where $\psi_{\mathrm{S}}$ and $\psi_{\mathrm{A}}$ are symmetric and antisymmetric wave-functions respectively. The electrostatic Coulomb interaction energy for the two electron system is then given by

$$
\begin{gathered}
V=\frac{1}{2} \int\left\{\psi_{\mathrm{a}}^{*}(1) \psi_{\mathrm{b}}^{*}(2) \pm \psi_{\mathrm{a}}^{*}(2) \psi_{\mathrm{b}}^{*}(1)\right\} \frac{\mathrm{e}^{2}}{\mathrm{r}_{12}}\left\{\psi_{\mathrm{a}}(1) \psi_{\mathrm{b}}(2) \pm \psi_{\mathrm{a}}(2) \psi_{\mathrm{b}}(1)\right\} \mathrm{d}^{3} \mathrm{r}_{1} \mathrm{~d}^{3} \mathrm{r}_{2} \\
=\frac{1}{2}\left\{\int\left|\psi_{\mathrm{a}}(1)\right|^{2} \frac{\mathrm{e}^{2}}{\mathrm{r}_{12}}\left|\psi_{\mathrm{b}}(2)\right|^{2} \mathrm{~d}^{3} \mathrm{r}_{1} \mathrm{~d}^{3} \mathrm{r}_{2}+\int\left|\psi_{\mathrm{a}}(2)\right|^{2} \frac{\mathrm{e}^{2}}{\mathrm{r}_{12}}\left|\psi_{\mathrm{b}}(1)\right|^{2} \mathrm{~d}^{3} \mathrm{r}_{1} \mathrm{~d}^{3} \mathrm{r}_{2}\right\} \\
\pm \frac{1}{2}\left\{\int \psi_{\mathrm{a}}^{*}(1) \psi_{\mathrm{b}}^{*}(2) \frac{\mathrm{e}^{2}}{\mathrm{r}_{12}} \psi_{\mathrm{a}}(2) \psi_{\mathrm{b}}(1) \mathrm{d}^{3} \mathrm{r}_{1} \mathrm{~d}^{3} \mathrm{r}_{2}\right. \\
\left. \pm \int \psi_{\mathrm{a}}^{*}(2) \psi_{\mathrm{b}}^{*}(1) \frac{\mathrm{e}^{2}}{\mathrm{r}_{12}} \psi_{\mathrm{a}}(1) \psi_{\mathrm{b}}(2) \mathrm{d}^{3} \mathrm{r}_{1} \mathrm{~d}^{3} \mathrm{r}_{2}\right\}
\end{gathered}
$$




$$
\begin{gathered}
=K \pm J_{e x} \\
K=\int\left|\Psi_{a}(1)\right|^{2} \frac{\mathrm{e}^{2}}{r_{12}}\left|\psi_{b}(2)\right|^{2} d^{3} r_{1} d^{3} r_{2} \\
J_{e x}=\int \psi_{\mathrm{a}}^{*}(1) \psi_{b}^{*}(2) \frac{e^{2}}{r_{12}} \psi_{a}(2) \psi_{b}(1) d^{3} r_{1} d^{3} r_{2}
\end{gathered}
$$

$\mathrm{K}$ and $\mathrm{J}_{\mathrm{ex}}$ are known as the Coulomb term and Exchange term, respectively. $\mathrm{J}_{\mathrm{ex}}$ is called exchange energy because the integral involves the exchange of the two electrons between site 1 and 2 . The Hamiltonian of the two electrons can then be written as:

$$
\mathrm{H}_{\mathrm{ab}}=\mathrm{K}_{\mathrm{ab}}-\frac{1}{2} \mathrm{~J}_{\mathrm{ex}}\left(1+4 \overrightarrow{\mathrm{S}_{\mathrm{a}}} \cdot \overrightarrow{\mathrm{S}_{\mathrm{b}}}\right)
$$

$$
\text { with } 2<\overrightarrow{\mathrm{S}_{\mathrm{a}}} \cdot \overrightarrow{\mathrm{S}_{\mathrm{b}}}>=\mathrm{S}(\mathrm{S}+1)-\mathrm{S}_{\mathrm{a}}\left(\mathrm{S}_{\mathrm{a}}+1\right)-\mathrm{S}_{\mathrm{b}}\left(\mathrm{S}_{\mathrm{b}}+1\right)
$$

For the triplet state total $\mathrm{S}=1, \mathrm{~S}_{\mathrm{a}}=\mathrm{S}_{\mathrm{b}}=1 / 2$ and so

$$
\begin{gathered}
2<\overrightarrow{\mathrm{S}_{\mathrm{a}}} \cdot \overrightarrow{\mathrm{S}_{\mathrm{b}}}>=2-\frac{3}{4}-\frac{3}{4}=\frac{1}{2} \\
\mathrm{H}_{\mathrm{ab}}=\mathrm{K}_{\mathrm{ab}}-\frac{1}{2} \mathrm{~J}_{\mathrm{ex}}\left(1+4 \overrightarrow{\mathrm{S}_{\mathrm{a}}} \cdot \overrightarrow{\mathrm{S}_{\mathrm{b}}}\right)=\mathrm{K}_{\mathrm{ab}}-\frac{1}{2} \mathrm{~J}_{\mathrm{ex}}\left(1+2 \cdot \frac{1}{2}\right)=\mathrm{K}_{\mathrm{ab}}-\mathrm{J}_{\mathrm{ex}}
\end{gathered}
$$

For singlet state total $\mathrm{S}=0, \mathrm{~S}_{\mathrm{a}}=\mathrm{S}_{\mathrm{b}}=1 / 2$ and so $2<\overrightarrow{\mathrm{S}_{\mathrm{a}}} \cdot \overrightarrow{\mathrm{S}_{\mathrm{b}}}>=0-\frac{3}{4}-\frac{3}{4}=-\frac{3}{2}$. This gives

$$
\mathrm{H}_{\mathrm{ab}}=\mathrm{K}_{\mathrm{ab}}-\frac{1}{2} \mathrm{~J}_{\mathrm{ex}}\left(1+4 \overrightarrow{\mathrm{S}_{\mathrm{a}}} \cdot \overrightarrow{\mathrm{S}_{\mathrm{b}}}\right)=\mathrm{K}_{\mathrm{ab}}-\frac{1}{2} \mathrm{~J}_{\mathrm{ex}}\left(1+2 \cdot \frac{-3}{2}\right)=\mathrm{K}_{\mathrm{ab}}+\mathrm{J}_{\mathrm{ex}}
$$


So for $\mathrm{J}_{\mathrm{ex}}>0$, the energy of triplet state is lower than the singlet state, corresponding to ferromagnetic state which neighboring spins are in the same direction; for $\mathrm{J}_{\mathrm{ex}}<0$, the energy of singlet state is lower than the triplet state, corresponding to antiferromagnetic state with neighboring spins are in opposite direction. Thus, from the above derivation, the Hamiltonian can be written as:

$$
\mathrm{H}_{\mathrm{ab}}=\mathrm{K}_{\mathrm{ab}}-\frac{1}{2} \mathrm{~J}_{\mathrm{ex}}\left(1+4 \overrightarrow{\mathrm{S}_{\mathrm{a}}} \cdot \overrightarrow{\mathrm{S}_{\mathrm{b}}}\right)=\mathrm{K}_{\mathrm{ab}}-\frac{1}{2} \mathrm{~J}_{\mathrm{ex}}-2 \mathrm{~J}_{\mathrm{ex}} \overrightarrow{\mathrm{S}_{\mathrm{a}}} \cdot \overrightarrow{\mathrm{S}_{\mathrm{b}}}
$$

The first two terms of the above equation are independent of the spin, whereas the last term depends on the spin. If $\mathrm{J}_{\mathrm{ex}}$ is negligible except for the nearest neighbors and is the same for all the nearest neighbors, then the spin-dependent Heisenberg exchange term can be written as:

$$
\mathrm{H}=-2 \mathrm{~J}_{e x} \sum \overrightarrow{\mathrm{S}_{1}} \cdot \overrightarrow{\mathrm{S}_{\mathrm{J}}}
$$

\subsection{The Bonner-Fisher Model for spin $S=1 / 2$}

Bonner and Fisher (BF) [5] considered linear magnetic chains in magnetic $\mathrm{H}$ with anisotropic

Heisenberg Hamiltonian:

$$
\boldsymbol{u}=-2 \mathrm{~J}_{\mathrm{ex}} \sum_{\mathrm{i}=1}^{\mathrm{N}}\left(\mathrm{S}_{\mathrm{i}}^{\mathrm{z}} \mathrm{S}_{\mathrm{i}+1}^{\mathrm{z}}\right)+\gamma\left(\mathrm{S}_{\mathrm{i}}^{\mathrm{x}} \mathrm{S}_{\mathrm{i}+1}^{\mathrm{x}}+\mathrm{S}_{\mathrm{i}}^{\mathrm{y}} \mathrm{S}_{\mathrm{i}+1}^{\mathrm{y}}\right)-\mathrm{g} \mu_{\mathrm{B}} \sum_{\mathrm{i}=1}^{\mathrm{N}} \mathbf{H} \cdot \mathbf{S}_{\mathbf{i}}
$$

For $\gamma=0$ (1), the Hamiltonian reduces to the Ising (Heisenberg) case. They solved this case for the calculations of magnetic susceptibility $\chi$ and specific heat $C_{M}$ for $N=2$ to 11 spins each with spin $S=1 / 2$ for both ferromagnetic $\left(\mathrm{J}_{\mathrm{ex}}>0\right)$ and antiferromagnetic $\left(\mathrm{J}_{\mathrm{ex}}<0\right)$ cases. For the antiferromagnetic case $\left(\mathrm{J}_{\mathrm{ex}}<\right.$ 
0) which applies to the $\mathrm{CuPc}$ and $\mathrm{CoPc}$ systems in this dissertation, these solution showed that for $\gamma=1$, the $\chi$ vs. $T$ data should go through a maximum at a temperature $T_{\max }$ given by

$$
\mathrm{T}_{\max } \approx 1.282 \frac{\left|\mathrm{J}_{\mathrm{ex}}\right|}{\mathrm{k}_{\mathrm{B}}}
$$

For $\gamma=0$ (Ising case), a similar maximum is predicted but at a temperature $T_{\max }=\left|\mathrm{J}_{\mathrm{ex}}\right| \mathrm{k}_{\mathrm{B}}$ (this will be discussed in the next section). Also the calculated magnitude of $\chi$ at $\mathrm{T}_{\max }$ is different for the two cases so that the difference between the $\gamma=0$ and $\gamma=1$ cases can be verified experimentally.

Since the BF solution was valid only for the maximum of $\mathrm{N}=11$ spins, a solution for $\mathrm{N} \rightarrow \infty$ valid for real systems was needed. Feyerherm et al. [6] provided such a solution valid for $T>0.1\left|\mathrm{~J}_{\mathrm{ex}}\right| / \mathrm{k}_{\mathrm{B}}$ and $\gamma=1$ in terms an empirical rational function for the magnetic susceptibility $\chi$ given by

$$
\begin{gathered}
\chi=\frac{C}{T} F(x) \\
F(x)=\frac{1+a_{1} x+a_{2} x^{2}}{1+b_{1} x+b_{2} x^{2}+b_{3} x^{3}}
\end{gathered}
$$

Here $C$ is the Curie constant, $x=\left|\mathrm{J}_{\mathrm{ex}}\right| /\left(\mathrm{k}_{\mathrm{B}} \mathrm{T}\right)$ and $\mathrm{a}_{1}=0.17032, \mathrm{a}_{2}=0.93404, \mathrm{~b}_{1}=1.46764, \mathrm{~b}_{2}=0.54784, \mathrm{~b}_{3}$ $=4.28544$. Eq. (2.51) can be rewritten as

$$
\frac{\chi\left|J_{e x}\right|}{\mathrm{Ck}_{\mathrm{B}}}=\mathrm{xF}(\mathrm{x})
$$

A plot of $\left(\chi\left|\mathrm{J}_{\mathrm{ex}}\right|\right) / \mathrm{Ck}_{\mathrm{B}}$ vs. $\mathrm{k}_{\mathrm{B}} \mathrm{T} /\left|\mathrm{J}_{\mathrm{ex}}\right|=1 / \mathrm{x}$ is shown in Fig. 2.4. The maxima occurs at $\mathrm{T}_{\max }=1.3\left|\mathrm{~J}_{\mathrm{ex}}\right| / \mathrm{k}_{\mathrm{B}}$ in agreement with the BF calculations; However, Feyerherm et al. showed that for $\mathrm{T}<0.5\left|\mathrm{~J}_{\mathrm{ex}}\right| / \mathrm{k}_{\mathrm{B}}$, there is 
some disagreement with the prediction of the BF model. In a later chapter, the experimental results of magnetic susceptibility versus temperature in $\alpha-\mathrm{CuPc}, \beta-\mathrm{CuPc}$ and $\beta-\mathrm{CoPc}$ are compared with Eq. (2.51) and Eq. (2.53).

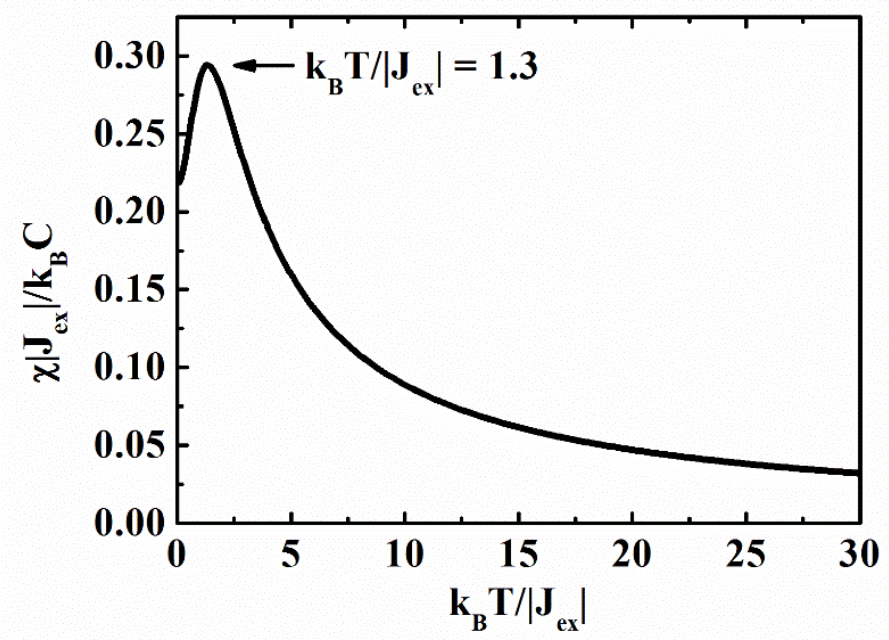

Fig. 2.4 Plot of the normalized parameters $\left(\chi\left|J_{e x}\right|\right) / C k_{B} v s . k_{B} T /\left|J_{e x}\right|=1 / x$.

\subsection{The Ising Chain Model for Spin S}

For Ising chain, the Hamiltonian is

$$
H=-2 J_{e x} \sum_{i=1}^{N} S_{i}^{z} S_{i+1}^{z}
$$

The parallel susceptibility (when the applied field is along z-axis) is given by C. Coulon et al. [7]

$$
\frac{\chi \mathrm{T}}{\mathrm{C}}=\frac{1+\Gamma}{1-\Gamma}
$$


Here as usual $\mathrm{C}=\mathrm{N} \mu^{2} / 3 \mathrm{k}_{\mathrm{B}}$ is the Curie constant and the correlation function $\Gamma=\tanh \left(2 \beta \mathrm{J}_{\mathrm{ex}} \mathrm{S}^{2}\right)$ with $\beta=$ $1 / \mathrm{k}_{\mathrm{B}} \mathrm{T}[8]$. For low temperature case, the parallel susceptibility is

$$
\frac{\chi_{/ /} \mathrm{T}}{\mathrm{C}} \approx \exp \left(4 \beta \mathrm{J}_{\mathrm{ex}} \mathrm{S}^{2}\right)
$$

It is noted that the perpendicular susceptibility of Ising chain has also been calculated and stays constant at low temperature remaining much smaller than the parallel susceptibility [9].For spin $S=1 / 2$ antiferromagnetic system $\left(\mathrm{J}_{\mathrm{ex}}<0\right)$, Eq. (2.56) can be written as

$$
\frac{\chi\left|\mathrm{J}_{\mathrm{ex}}\right|}{\mathrm{Ck}_{\mathrm{B}}}=\mathrm{x} e^{-\mathrm{x}}
$$

where $x=\left|J_{e x}\right| / k_{B} T$. A plot of the normalized parameters $\left(\chi\left|J_{e x}\right|\right) / C k_{B}$ vs. $k_{B} T /\left|J_{e x}\right|=1 / x$ is shown in Fig. 2.5. The maxima occurs at $\mathrm{T}_{\max }=1.0\left|\mathrm{~J}_{\mathrm{ex}}\right| / \mathrm{k}_{\mathrm{B}}$ in agreement with the $\mathrm{BF}$ calculations.

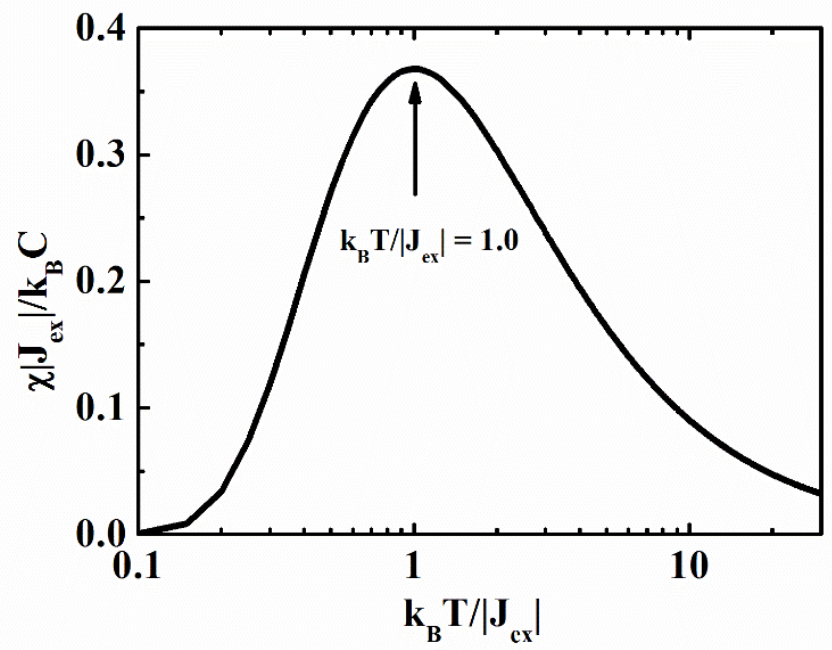

Fig. 2.5 Plot of $\left(\chi\left|J_{e x}\right|\right) / C k_{B}$ vs. $k_{B} T /\left|J_{e x}\right|=1 / x$ for Ising chain with spin $S=1 / 2$ antiferromagnetic coupled system. 
The theoretical expression for the magnetic field dependence of the magnetization of an Ising chain magnet has been given as $[10,11]$ :

$$
\frac{\mathrm{M}}{\mathrm{M}_{\mathrm{S}}}=\frac{\sinh \left(\mu_{\mathrm{B}} \beta \mathrm{H}\right)}{\sqrt{\sinh ^{2}\left(\mu_{\mathrm{B}} \beta \mathrm{H}\right)+\exp \left(-8 \beta \mathrm{J}_{\mathrm{ex}} \mathrm{S}^{2}\right)}}
$$

In Eq. (2.58), $M_{S}$ is the saturation magnetization and $\beta=1 / k_{B} T$. In the following chapters, the theoretical expressions given above for the Ising and Heisenberg chain models will be tested against the experimental results of the temperature and magnetic field dependence of the magnetization measured in powder samples of TMPc $(\mathrm{TM}=\mathrm{VO}, \mathrm{Mn}, \mathrm{Co}$ and $\mathrm{Cu})$. These new experimental results and their theoretical interpretations based on the above models are the new contributions of this dissertation.

\section{References}

[1] P. Langevin, Ann. Chemie et Physique. 5, 70 (1905).

[2] P. Weiss, J. de Physique. 6, 66 (1907).

[3]. A. H. Morrish. The Physical Principals of Magnetism (IEEE Press, New York. 2001).

[4] G.E. Pake. Paramagnetic Resonance (W.A. Benjamin. Inc. New York, 1962).

[5] J.C. Bonner and M.E. Fisher, Phys. Rev. 135, A640 (1964).

[6] R. Feyerhem, S. Abens, D. Gunther, T. Ishida, M. Meibner, M. Meschke, T. Nogami and M. Steiner, J. 
Phys. Condens. Matter. 12, 8495 (2000).

[7] C. Coulon, H. Miyasaka and R. Clerac, Struct. Bond. 122, 163 (2006).

[8] E. Ising, J. Math. Phys. 4, 124 (1963).

[9] M.E. Fisher, J. Math. Phys. 4, 124 (1963).

[10] M. Steiner, J. Villain and C.G. Windsor, Adv. Phys. 25, 87 (1976).

[11] F. Boersma, W.J.M. de Jonge, and K. Kopinga, Phys. Rev. B. 23, 186 (1981). 


\section{CHAPTER 3.}

\section{Experimental Techniques}

\subsection{Introduction}

In this chapter, relevant details of the experimental techniques used for the structural and magnetic characterization of the TMPc samples are described. Most of the samples used in these investigations were purchased from Alfa Aesar and Sigma Aldrich. The exception was the sample of $\beta$-CuPc which was converted from the commercial $\alpha$-CuPc using thermo-gravimetric analysis (TGA). All the samples were characterized by X-ray diffraction (XRD) and scanning electron microscopy (SEM) in order to acquire the information on the crystalline phases and morphology of the samples. The major portion of magnetization measurements in this dissertation were performed by using the AC Measurement System (ACMS) option of our in-house Physical Property Measurement System (PPMS) manufactured by Quantum Design Inc. Therefore some details of the techniques of TGA, XRD, SEM and ACMS are described below.

\subsection{Thermo-gravimetric Analysis}

Thermo-gravimetric Analysis (TGA) is a technique in which the mass of a substance is monitored as a function of temperature or time as the sample specimen is subjected to a controlled temperature program in a controlled atmosphere. TGA can provide useful information on both the physical and chemical properties of a sample, including thermal stability, oxidative stability, composition, estimated lifetime and decomposition kinetics [1]. In addition, TGA is an important technique for material 
characterization for various environmental, food, pharmaceutical, and petrochemical industries [2-4].

In principle, TGA characterizes materials that exhibit weight loss or gain due to decomposition, oxidation, and dehydration. The reasons for weight loss in a material include decomposition, evaporation, reduction, and desorption. Materials can also gain weight during oxidation and absorption. Combining data provided by TGA with proper data analysis, the kinetics of various processes can also be studied.

The essential parts of TGA (shown in Fig. 3.1) are a furnace with controlled gas flow, a balance with a sample pan, and a computer. The furnace houses the sample (in a sample pan) and can provide the temperature from $20{ }^{\circ} \mathrm{C}$ up to $1000{ }^{\circ} \mathrm{C}$ with a controlled heating rate, normally $0.1{ }^{\circ} \mathrm{C} / \mathrm{min}$ to $100{ }^{\circ} \mathrm{C} / \mathrm{min}$. The gas is normally nitrogen. The balance can measure the sample weight precisely and the sensitivity can reach $0.1 \mu \mathrm{g}$. The sample pan is made of Platinum, Aluminum, or ceramics, and its usage depends on the application. The computer controls all the programs and performs data analysis.

\section{instrumentation}

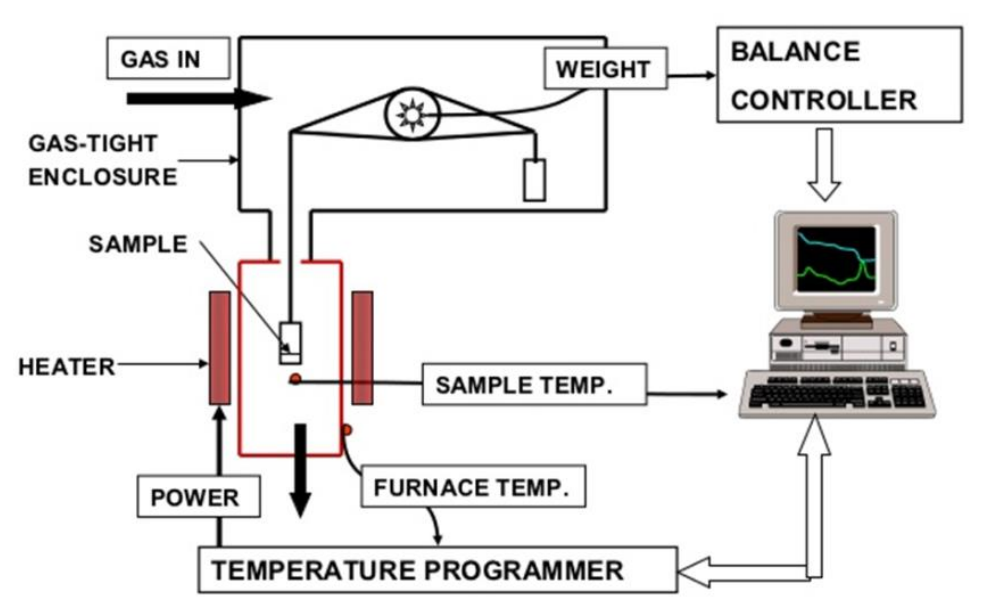

Fig. 3.1 Instrumentation of TGA (adapted from [5]). 
In this research, TGA was used to convert to convert $\alpha-\mathrm{CuPc}$ to $\beta$-CuPc by annealing it at $350{ }^{\circ} \mathrm{C}$ in nitrogen environment. The details of these results are described in Chapter 4. A Q50 TGA manufactured by TA Instruments was used for this purpose (see Fig. 3.2). About $30 \mathrm{mg} \alpha$-CuPc powder sample was loaded onto a platinum pan and put in the furnace. After accurately weighing the sample, the temperature in the furnace was increased from $20^{\circ} \mathrm{C}$ up to $350{ }^{\circ} \mathrm{C}$ with the heating rate of $10{ }^{\circ} \mathrm{C} / \mathrm{min}$ and kept at $350{ }^{\circ} \mathrm{C}$ for 3 hours. The annealing process was done in the $\mathrm{N}_{2}$ environment by flowing $\mathrm{N}_{2}$ gas. The weight vs. temperature and weight vs. time data are shown in Fig. 4.1 of Chapter 4 and its analysis is discussed in section 4.2.1.

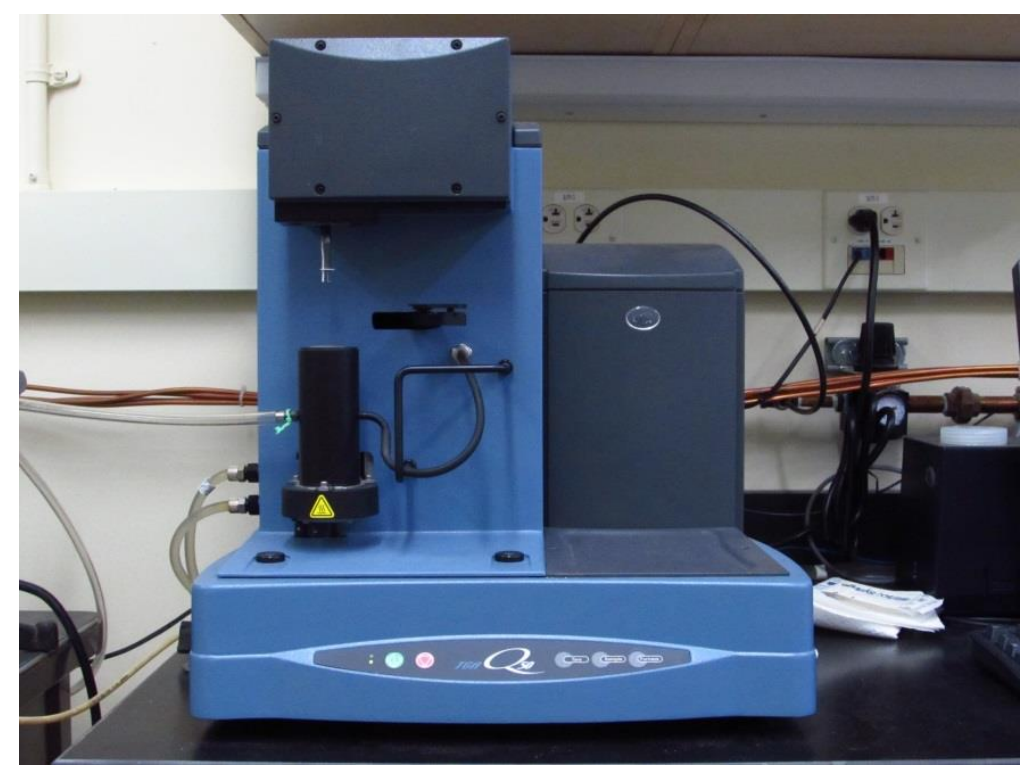

Fig. 3.2 A photograph of the commercial Q50 TGA system used for experiments.

\subsection{X-ray Diffraction}

\subsection{1: History and Production of X-rays}

The discovery of X-ray in 1895 by Roentgen enabled scientists to probe crystalline structure at 
the atomic level and the first diffraction pattern of a crystal was made by Knipping and von Laue in 1914. Soon after this, in 1915 Bragg developed the theory to determine the crystal structure from Bragg diffraction. Nowadays with the help of the high performance computers, X-ray diffraction (XRD) has become an essential tool for the determination of crystal structures and hence it is used extensively in academic laboratories as well as in medical, dental and pharmaceutical industries.

Solid matter can be described as amorphous or crystalline. For amorphous materials, the atoms are arranged in a random manner similar to the atomic disorder found in liquids. However, for crystalline materials, the atoms are arranged in a regular pattern which can be replicated from the unit cell of the structure. In three dimensions, the unit cell is defined by a-, b-, c-axis with angles between each two axes are called $\alpha, \beta$, and $\gamma$. As an example, for a cubic unit cell $\mathrm{a}=\mathrm{b}=\mathrm{c}$ and $\alpha=\beta=\gamma=90^{\circ}$. For basics of unit cells, x-ray diffraction and x-ray crystallography, the book by Cullity is a good source [6]. So far, diffraction patterns have been collected for about 50,000 inorganic and 25,000 organic single component, crystalline phases, which enable researchers to identify components in a sample using a search/match procedure.

X-rays are a part of the electromagnetic radiation with the wavelength from $0.01 \mathrm{~nm}$ to $10 \mathrm{~nm}$ (frequency from $3 \times 10^{16}$ to $3 \times 10^{19} \mathrm{~Hz}$ ) depending on the source. As a result, the $\mathrm{X}$-rays carry high energy photons (from $120 \mathrm{eV}$ to $120 \mathrm{keV}$ ) and are quite penetrating. The relation of energy, wavelength, and frequency of a photon is as follows:

$$
\mathrm{E}=\mathrm{h} v=\frac{\mathrm{hc}}{\lambda}
$$


In Eq. (3.1), $\mathrm{E}$ is the photon energy, $\mathrm{h}$ is Planck constant, $\mathrm{c}$ is speed of light, and $\lambda$ is the wavelength.

X-rays are commonly produced by bombarding a metal target (e.g. $\mathrm{Cu}$, Mo) with a beam of high-speed electrons (accelerated by high voltage) emitted from a hot filament (often tungsten), as shown in Fig. 3.3. The incident beam will ionize electrons from the K-shell (1s) of the target atom and X-rays are emitted as the resultant vacancies are filled by electrons dropping down from the L (2p) or M (3p) levels. This gives rise to $\mathrm{K} \alpha$ and $\mathrm{K} \beta$ lines (see Fig. 3.4 and Fig.3.5). For example, the wavelengths of $\mathrm{Cu}-\mathrm{K} \alpha$ and Mo-K $\alpha$ are $0.15418 \mathrm{~nm}$ and $0.07107 \mathrm{~nm}$, respectively. In practical x-ray systems, $\mathrm{K} \beta$ lines are usually absorbed by a filter so that nearly monochromatic source of $\mathrm{x}$-rays is available for experiments.

The wavelength of the emitted $\mathrm{X}$-rays with applied voltage $\mathrm{V}$ is given by:

$$
\lambda=\frac{\mathrm{hc}}{\mathrm{E}}=\frac{\mathrm{hc}}{\mathrm{eV}}=\frac{\left(6.626 \times 10^{34}\right)\left(3 \times 10^{8}\right)\left(10^{9}\right)}{\left(1.6 \times 10^{-19}\right) \mathrm{V}}=\frac{1.24}{\mathrm{~V}(\mathrm{kV})} \mathrm{nm}
$$

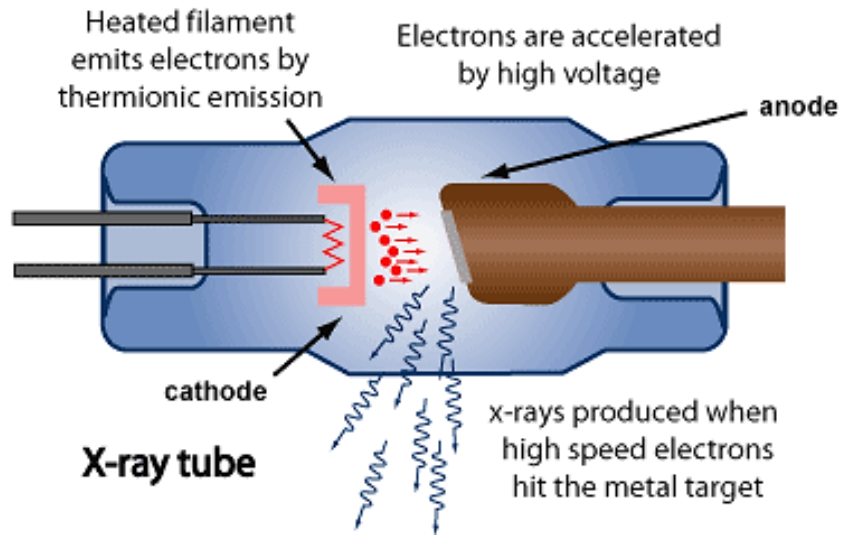

Fig. 3.3 Schematic diagram of X-ray tube (adapted from [7]).

In 1913, Moseley was the first person to show that the frequencies $v$ of X-rays emitted from 
chemical elements are proportional to the square of a number which was close to the element's atomic number Z. Specifically this relation (Moseley's law) is given by $v=K(Z-\sigma)^{2}$ where $K$ is a universal constant for all elements and $\sigma$ is another universal constant representing electron screening effect. Soon after Moseley's discovery, in 1914 it was realized that Moseley's formula could be adapted from Bohr's equation, as following:

$$
\begin{gathered}
E=h v=E_{i}-E_{f}=\frac{m_{e} q_{e}^{4}(Z-\sigma)^{2}}{8 h^{2} \varepsilon_{0}^{2}}\left(\frac{1}{n_{f}^{2}-n_{i}^{2}}\right) \\
v=\frac{m_{e} q_{e}^{4}(Z-\sigma)^{2}}{8 h^{3} \varepsilon_{0}^{2}}\left(\frac{1}{n_{\mathrm{f}}^{2}-n_{i}^{2}}\right)
\end{gathered}
$$

Here $\mathrm{m}_{\mathrm{e}}$ is the mass of electron, $\mathrm{q}_{\mathrm{e}}$ is the charge of electron, $\mathrm{Z}$ is the atomic number, $\varepsilon_{0}$ is the permittivity of the vacuum, $\mathrm{n}_{\mathrm{f}}$ and $\mathrm{n}_{\mathrm{i}}$ are final and initial energy level, respectively.

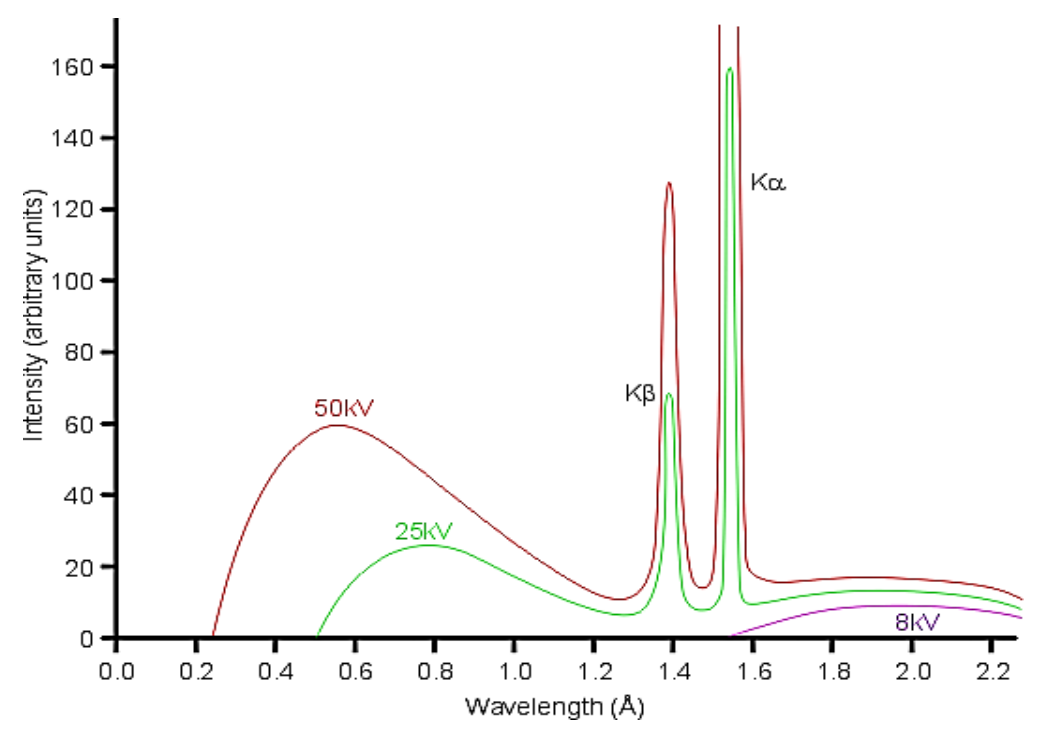

Fig. 3.4 Intensity vs. wavelength of X-rays for three applied voltages (adapted from [8]). 


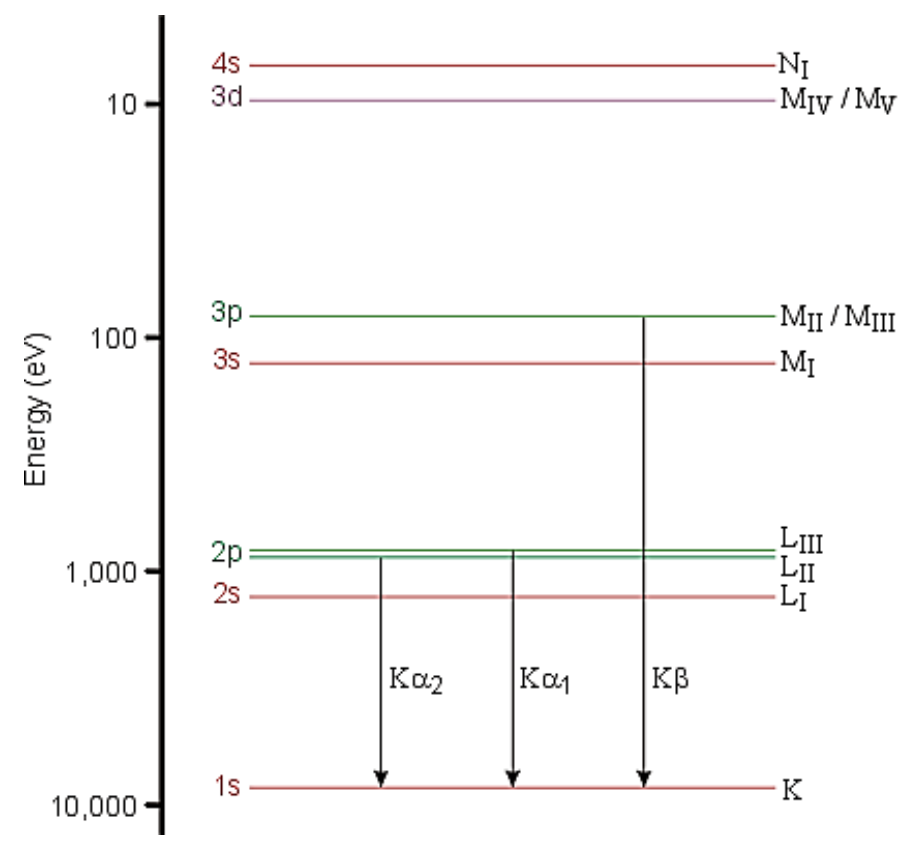

Fig. 3.5 Energy level involved in CuK X-ray emission (adapted from [7]).

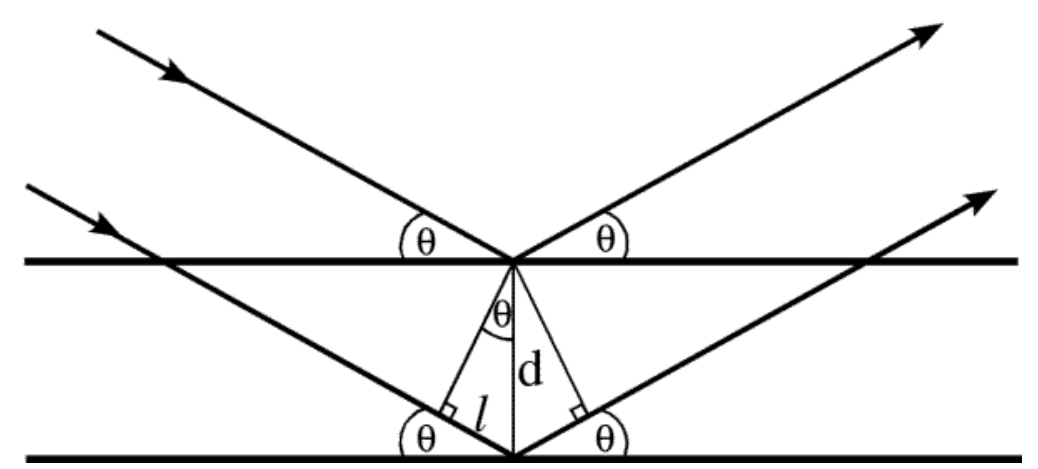

Fig. 3.6 Diffraction of X-rays from two parallel containing atoms (adapted from [9]).

\subsubsection{Bragg's Law and Determination of Crystal Structures:}

Consider an x-ray beam of wavelength $\lambda$ incident on a pair of parallel planes, separated by an inter-planar spacing d, as shown in Fig.3.6. The diffraction of X-ray is governed by Bragg's law: 


$$
2 \mathrm{~d} \sin \theta=\mathrm{n} \lambda
$$

Here $\theta$ is the incident angle and $n=1,2,3$--- gives the order of diffraction. The various observed Bragg peaks as a function of the diffraction angle can be indexed by Miller indices (hkl) representing various planes. For the TMPc samples, analysis of such data is presented in the subsequent chapters for each sample. This analysis requires comparing the measured d-values determined from Eq. (3.5) and comparing them with the d-values calculated for the expected structure. The d-spacings of triclinic structure in terms of the Miller indices (hkl) is given by [6]:

$$
\frac{1}{\mathrm{~d}^{2}}=\frac{1}{\mathrm{~V}^{2}}\left(\mathrm{~S}_{11} \mathrm{~h}^{2}+\mathrm{S}_{22} \mathrm{k}^{2}+\mathrm{S}_{33} \mathrm{l}^{2}+2 \mathrm{~S}_{12} \mathrm{hk}+2 \mathrm{~S}_{23} \mathrm{kl}+2 \mathrm{~S}_{13} \mathrm{hl}\right)
$$

with

$$
\begin{gathered}
S_{11}=b^{2} c^{2}(\sin \alpha)^{2} \\
S_{22}=a^{2} c^{2}(\sin \beta)^{2} \\
S_{33}=a^{2} b^{2}(\sin \gamma)^{2} \\
S_{12}=a b c^{2}(\cos \alpha \cos \beta-\cos \gamma) \\
S_{23}=a^{2} b c(\cos \beta \cos \gamma-\cos \alpha) \\
S_{13}=a b^{2} c(\cos \gamma \cos \alpha-\cos \beta)
\end{gathered}
$$

Here $\alpha, \beta, \gamma$ are angles between a-b axes, b-c axes, and a-c axes respectively. The volume $\mathrm{V}$ is given by 


$$
\mathrm{V}=\mathrm{abc} \sqrt{1-(\cos \alpha)^{2}-(\cos \beta)^{2}-(\cos \gamma)^{2}-2 \cos \alpha \cos \beta \cos \gamma}
$$

For the monoclinic structure, $\alpha=\gamma=90^{\circ}$ and so Eq. (3.6) becomes

$$
\frac{1}{\mathrm{~d}^{2}}=\frac{1}{\sin ^{2} \beta}\left(\frac{\mathrm{h}^{2}}{\mathrm{a}^{2}}+\frac{\mathrm{k}^{2} \sin ^{2} \beta}{\mathrm{b}^{2}}+\frac{\mathrm{l}^{2}}{\mathrm{c}^{2}}-\frac{2 \mathrm{hl} \cos \beta}{\mathrm{ac}}\right)
$$

In a similar manner, relations for d-spacing of other crystal structures is easily derived using the general equations given above. For the samples of $\alpha$-CuPc, $\beta-\mathrm{CuPc}, \beta-\mathrm{CoPc}, \beta-\mathrm{NiPc}$, and $\beta-\mathrm{MnPc}$ investigated here, the crystal structure is monoclinic whereas the crystal structure of for VOPc (phase II) is triclinic.

For all the samples investigated in this work, the XRD patterns were acquired at room temperature using the PANalytical X'Pert Pro X-ray difractometer with $\mathrm{Cu}-\mathrm{K} \alpha$ source $(\lambda=0.15418 \mathrm{~nm})$ available from the WVU Shared Research Facilities. The patterns of the scattered x-ray intensity versus scattering angle $2 \theta$ were obtained using a step size of $0.05^{\circ}$ and an integration time of $1.20 \mathrm{~s}$ for each step. The XRD generator was set to $40 \mathrm{~mA}$ and $45 \mathrm{kV}$. The analysis and discussion of the XRD data of all the investigated here is given in subsequent chapters along with the presentation and discussion of their magnetic properties.

\subsection{Scanning Electron Microscopy (SEM)}

At the request of the author and Professor Seehra, scanning electron microscopy of the TMPc powder samples was done by Mr. James Poston of the National Energy Technology Laboratory, U.S. Department of Energy, located in Morgantown, WV. According to the information provided by Mr. Poston, he used a JEOL 7600 FE-SEM system available in his laboratory. A brief summary of the working 
principles of SEM is given below.

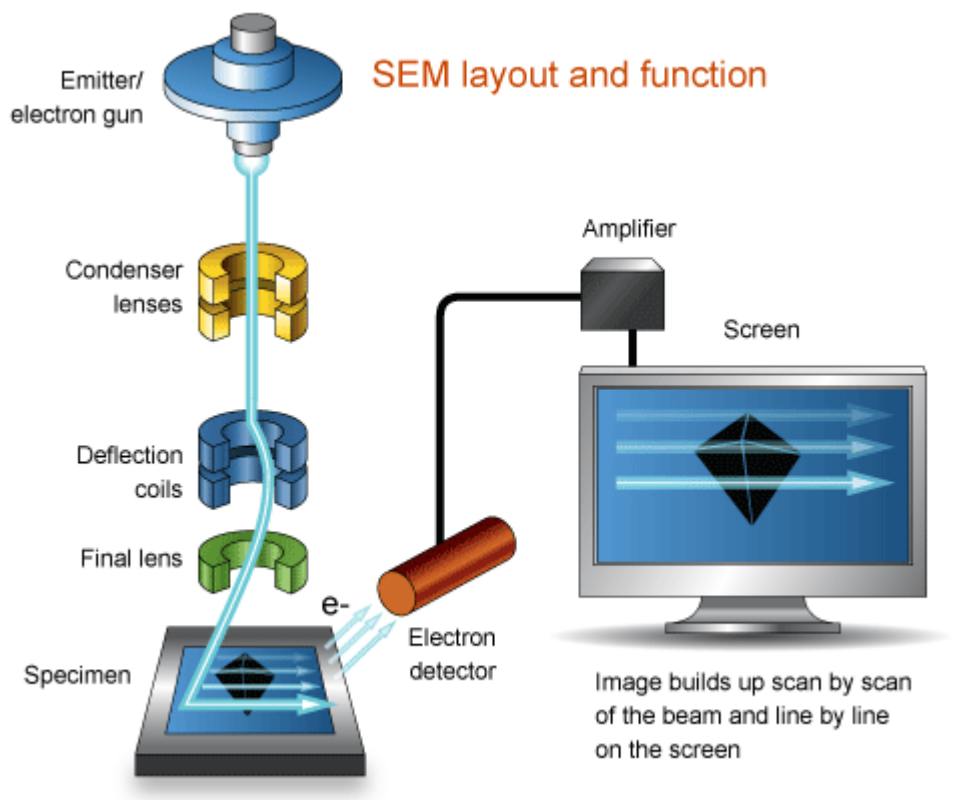

Fig. 3.7 Schematic diagram of the key components of SEM systems (adapted from [10]).

A scanning electron microscope (SEM) uses electrons, rather than light, to create the magnified image. Since first commercialization of SEM about 40 years ago, good quality SEM systems have now magnifications of up to $10^{5}$ with a resolution of $1 \mathrm{~nm} \sim 2 \mathrm{~nm}$. When a specimen is irradiated with a fine electron beam (called an electron probe), secondary electrons are emitted from the specimen surface. Topography of the surface can then be observed by two-dimensional scanning the electron probe over the surface and acquisition of an image from the detected secondary electrons.

A schematic diagram of the instrumentation of SEM is shown in Fig. 3.7. The electron gun produces an electron beam by heating the filament (usually Tungsten) at high temperatures and the electron beam passes through a high voltage (1 keV to $30 \mathrm{keV})$ region which accelerates the electrons. Then the high energy electron beam passes through condenser lenses, deflection coils, and final lens in 
order to adjust the diameter of the beam as well as getting a focused beam. When the incident beam enters the specimen, secondary electrons are produced from the emission of the valence electrons of the atoms in the specimen. Since the energy of secondary electrons is very small, those generated at a deep region are quickly absorbed by the specimen. Only those generated at the top of the specimen are emitted outside of it and as a result, the secondary electrons are very sensitive to the surface. Finally the secondary electrons are collected by the electron detector, amplified, and sent to the computer for data analysis. In addition, the inside of the electron optical system and specimen chamber must be kept at a high vacuum $\left(10^{-3} \mathrm{~Pa}\right.$ to $10^{-4} \mathrm{~Pa}$ ). This is because the chemical and thermal stability is necessary for a well-functioning filament (gun pressure). Also, the electron beam must travel to the specimen and secondary electrons must travel from the specimen to the detector without colliding with other atoms or molecules.

\subsection{Specifics of the AC Measurement System (ACMS)}

The ACMS option for the in-house PPMS acquired from Quantum Design Inc. is a versatile DC magnetometer and $\mathrm{AC}$ susceptometer. The available temperature range is from $2 \mathrm{~K}$ to $350 \mathrm{~K}$ in the sample region with temperature resolution stability of $0.1 \mathrm{~K}$. The available magnetic field is up to $\pm 90 \mathrm{kOe}( \pm 9$ $\mathrm{T})$ for DC magnetization measurement and up to \pm 10 Oe for the AC magnetization measurement in the

frequency range from $10 \mathrm{~Hz}$ to $10^{4} \mathrm{~Hz}$. As shown in Fig. 3.8, the ACMS insert houses the drive and detection coils, thermometer, and electrical connections for the ACMS system. Note that the detection coils consist two sets of counter-wound copper coils connected in series and separated by several centimeters. The sample is held within the insert's coil set on the end of a thin, rigid sample rod and can be translated longitudinally by a DC servo motor located in the ACMS sample transport assembly. The 
DC servo motor provides rapid and very smooth longitudinal sample motion. The system does not use a lock-in amplifier but a digital signal processor (DSP) that synthesizes the excitation signal and processes the detection coils' response signal.

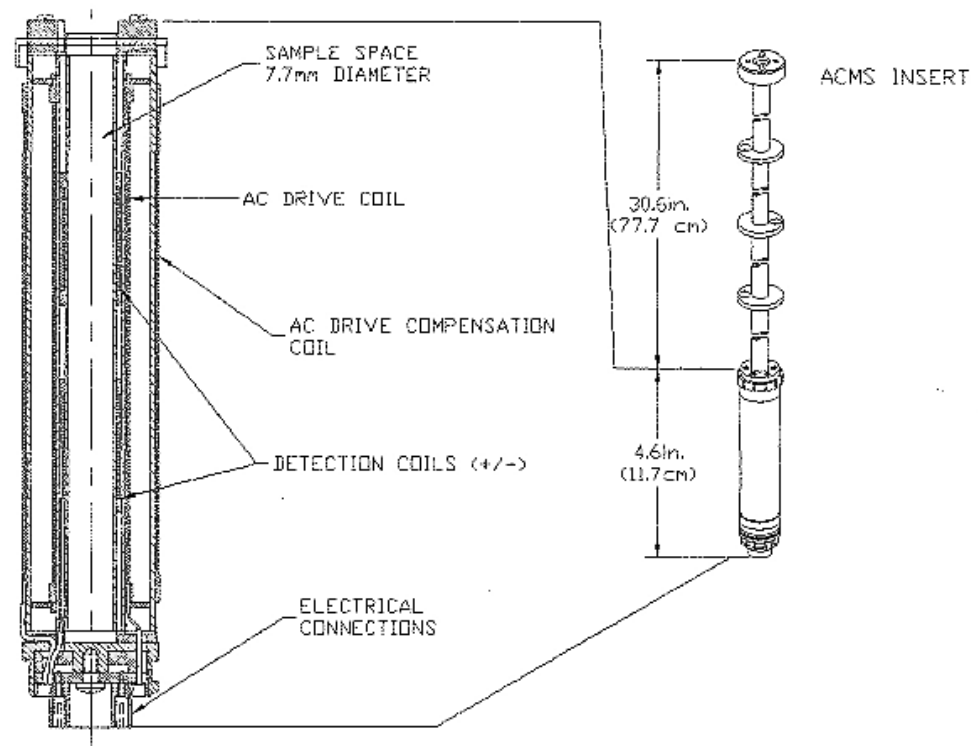

Fig. 3.8 ACMS insert and coil set (adapted from [11]).

DC magnetization measurements measure a sample's magnetic moment $\mathrm{M}$ in an applied field $\mathrm{H}$ at a specific temperature $\mathrm{T}(\mathrm{M}=\mathrm{M}(\mathrm{H}, \mathrm{T}))$. During $\mathrm{DC}$ magnetization measurements, a constant field (generated by the PPMS superconducting magnet) is applied to the measurement region and the sample is moved automatically and very quickly in $0.05 \mathrm{sec}(\mathrm{v} \approx 100 \mathrm{~cm} / \mathrm{s})$ through both sets of the detection coils, introducing a signal (induced emf) in them according to Faraday's law:

$$
\varepsilon=-\frac{1}{\mathrm{c}} \frac{\partial \Phi}{\partial \mathrm{t}}
$$

Here $\varepsilon$ is the induced emf and $\Phi$ is the magnetic flux which is proportional to the sample's magnetic 
moment and coil geometry. This measurement method is commonly called the extraction method. The system is calibrated using a standard sample of pure Pd (supplied by Quantum Design Inc.) with temperature and magnetic field independent magnetic susceptibility $\chi=5.24 \times 10^{-6} \mathrm{emu} / \mathrm{gOe}$.

AC magnetization measurements do not directly measure a sample's magnetic moment; instead, the change of the magnetic moment is measured:

$$
\chi_{\mathrm{ac}}=\frac{\mathrm{dM}}{\mathrm{dH}}
$$

This is the local slope of the sample's magnetization curve $\mathrm{M}(\mathrm{H})$ and not the "true" susceptibility. During AC magnetization measurements, an alternating field (up to \pm 10 Oe and frequency between $10 \mathrm{~Hz}$ and $\left.10^{4} \mathrm{~Hz}\right)$ is generated by the AC drive coil and applied to the measurement region. The sample is positioned in the center of the detection coils and the detection coils indicate how the applied field is altered by the presence of the sample.

There are many factors to consider when determining whether to use AC or DC magnetization measurements. For the ACMS option, AC magnetization measurements are nearly three orders of magnitude more sensitive than DC magnetization measurements. Conductive samples usually yield much larger signals during AC magnetization measurements. However, in some situations, DC magnetization measurements produce better results than $\mathrm{AC}$ magnetization measurements. For example, weakly paramagnetic samples with magnetic moments near the noise floor of the ACMS's AC measurement capability $\left(\sim 1 \times 10^{-8}\right.$ emu $)$ can often be detected more easily in the large DC fields applied by the PPMS superconducting magnet, because the larger DC field raises the sample's moment well above the noise 
floor for DC measurements $\left(\sim 2.5 \times 10^{-5} \mathrm{emu}\right)$.

A computer sequence is needed for each set of magnetic measurements. Examples of such sequences generated by the author for three typical experiments are given in Appendix B.

\section{References}

[1] A.W. Coats and J.P. Redfern, Analyst. 88, 906 (1963).

[2] D. Bom, R. Andrews, D. Jacques, J. Anthony, B. Chen, M.S. Meier, and J.P. Selegue, Nano Lett. 2, 615 (2002).

[3] U.K. Sharma, A. Verma, S.K. Prajapati, H. Pandey, and A.C. Pandey, Appl. Nanosci. 5, 143 (2015).

[4] I. Blanco and V. Siracusa, J. Therm. Anal. Calorim. 112, 1171 (2013).

[5] http://www.slideshare.net/nimmidalwadi5/tga

[6]. B. D. Cullity, "Elements of X-Ray Diffraction” (Addison-Wesley Publishing Co., Reading, MA 1959)

[7] http://www.arpansa.gov.au/radiationprotection/basics/xrays.cfm

[8] http://pd.chem.ucl.ac.uk/pdnn/inst1/xrays.htm

[9] http://www-structmed.cimr.cam.ac.uk/Course/Basic_diffraction/Diffraction.html

[10] http://www.ammrf.org.au/myscope/sem/practice/principles/layout.php\#detail

[11] PPMS ACMS User's Manual, Quantum Design Inc. 


\section{CHAPTER 4.}

\section{Magnetic Properties of $\alpha$ - and $\beta$ - Copper Phthalocyanines}

\subsection{Introduction}

In this chapter, results from the measurements of the magnetization $(\mathrm{M})$ of $\alpha-\mathrm{CuPc}$ and $\beta-\mathrm{CuPc}$ samples are presented covering the temperature range of $2 \mathrm{~K}$ to $250 \mathrm{~K}$ and in magnetic fields up to 90 kOe available with the in-house commercial PPMS. The data are analyzed and discussed in terms of Curie-Weiss $(\mathrm{CW})$ law and the Bonner-Fisher (BF) model for a Heisenberg linear chain. The results are also compared to the previous investigations of these materials [1], which did not cover such large ranges of temperatures and magnetic fields. A major portion of the results presented in this chapter have been published by the author in a recent paper [2].

\subsection{Samples and their Structural Characterization}

\subsubsection{Samples}

The CuPc powder was purchased from Alfa Aesar (stock \#43650) and confirmed as $\alpha$-phase by $\mathrm{x}$-ray diffraction (shown later). The structure of $\beta$-CuPc is more stable thermally than that of $\alpha$-CuPc since $\beta$-CuPc can be obtained by annealing $\alpha$-CuPc in $\mathrm{N}_{2}$ gas at $350^{\circ} \mathrm{C}$ for 3 hours as reported by Lozzi et al. [3]. This experiment was performed by using the in-house TGA (Thermo-gravimetric Analysis) system, model Q50 manufactured by TA Instruments Inc. The sample of $\alpha$-CuPc powder was placed on the pan balance which was further lowered into the furnace of the TGA system and heated from $20{ }^{\circ} \mathrm{C}$ to $350{ }^{\circ} \mathrm{C}$ at a heating rate of $10{ }^{\circ} \mathrm{C} / \mathrm{min}$. The $\mathrm{N}_{2}$ gas was introduced into the furnace while the sample was heating 
in order to keep the environment inert. With increase in temperature, the sample weight decreases by a few percent most likely due to the loss of adsorbed moisture in the sample. Once the temperature reached $350{ }^{\circ} \mathrm{C}$, there was no further loss in the weight as evident in figure $4.1(\mathrm{a})$. The sample was kept at $350{ }^{\circ} \mathrm{C}$ for 3 hours followed by cooling it to room temperature in flowing $\mathrm{N}_{2}$ gas. X-ray diffraction of this annealed sample confirmed it to have the $\beta$-phase of $\mathrm{CuPc}$ as shown in the following section.
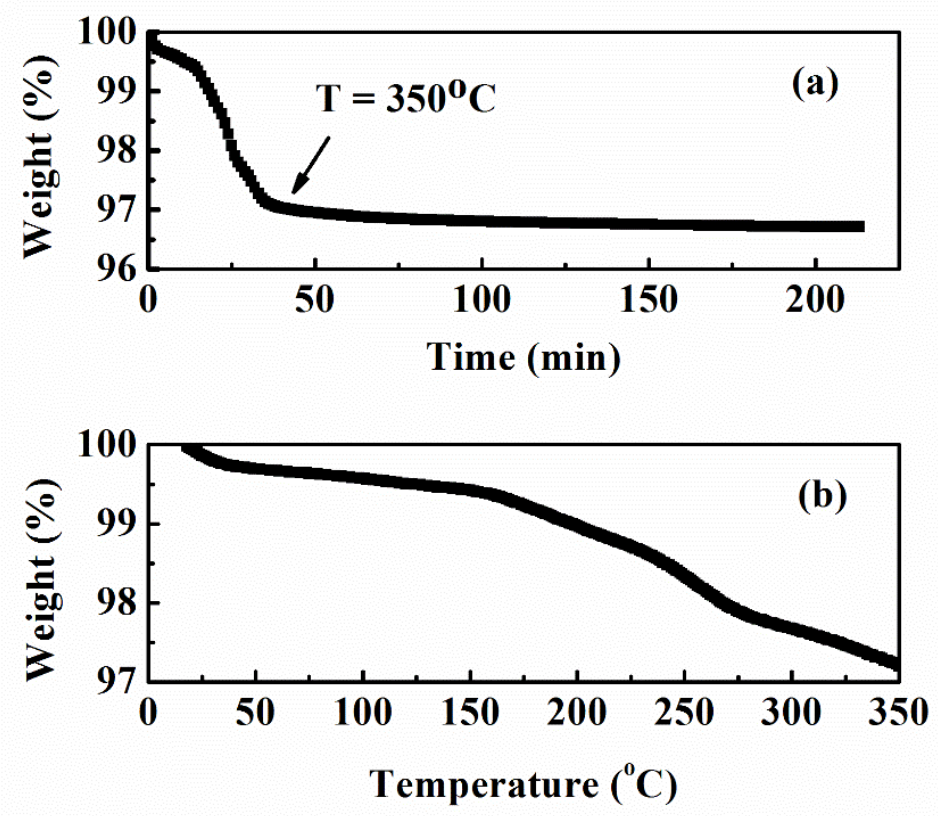

Fig. 4.1 Plots of weight vs. time (a) and weight vs. temperature (b) of $\alpha$-CuPc powder sample during the annealing process. The temperature of sample was varied from $20^{\circ} \mathrm{C}$ to $350{ }^{\circ} \mathrm{C}$ at a heating rate of $10{ }^{\circ} \mathrm{C} / \mathrm{min}$ in flowing $\mathrm{N}_{2}$ gas, then kept constant at $350{ }^{\circ} \mathrm{C}$ for three hours.

\subsubsection{X-ray Diffraction (XRD)}

The x-ray diffraction (XRD) of the purchased powder sample was performed at room temperature using a PANalytical X'Pert Pro X-ray Diffractometer with monochromatic $\mathrm{Cu}-\mathrm{K} \alpha$ radiation of wavelength $\lambda=0.15418 \mathrm{~nm}$ (Fig.4.2). The analysis of the XRD pattern showed the structure to match 
perfectly with that of $\alpha$-CuPc having space group $\mathrm{C} 2 / \mathrm{c}$ with four molecules per unit cell of the monoclinic structure and lattice parameters: $a=2.592 \mathrm{~nm}, \mathrm{~b}=0.379 \mathrm{~nm}, \mathrm{c}=2.392 \mathrm{~nm}$, and $\beta=90.4^{\circ}$ $[4,5]$. The XRD of the annealed sample, also shown in Fig.4.2, confirmed the conversion to the $\beta$-phase having space group $\mathrm{P} 2 / \mathrm{a}$ and two molecules per unit cell of the monoclinic structure with lattice parameters: $\mathrm{a}=1.9407 \mathrm{~nm}, \mathrm{~b}=0.479 \mathrm{~nm}, \mathrm{c}=1.4628 \mathrm{~nm}$, and $\beta=120.56^{\circ}[3,6,7]$. Note that all the observed lines in Fig. 4.2 for both phases could be indexed using the respective parameters listed above although the Miller indices of only the most prominent lines are shown in Fig. 4.2 to avoid over-crowding the figures. The unit cells of $\alpha$-and $\beta$-phases are shown in Fig. 1.1(chapter 1) with magnitudes of the cell parameters listed in Table 1.1. Note that the $\mathrm{Cu}-\mathrm{Cu}$ chain is along the b-axis with $\mathrm{Cu}-\mathrm{Cu}$ distance of 0.379 $\mathrm{nm}(0.479 \mathrm{~nm})$ and the stack angle $\delta=65^{\circ}\left(45^{\circ}\right)$ for the $\alpha(\beta)$ phase [8]. These structural differences are used later to interpret the observed differences in the measured magnetic properties of the two phases of $\mathrm{CuPc}$.

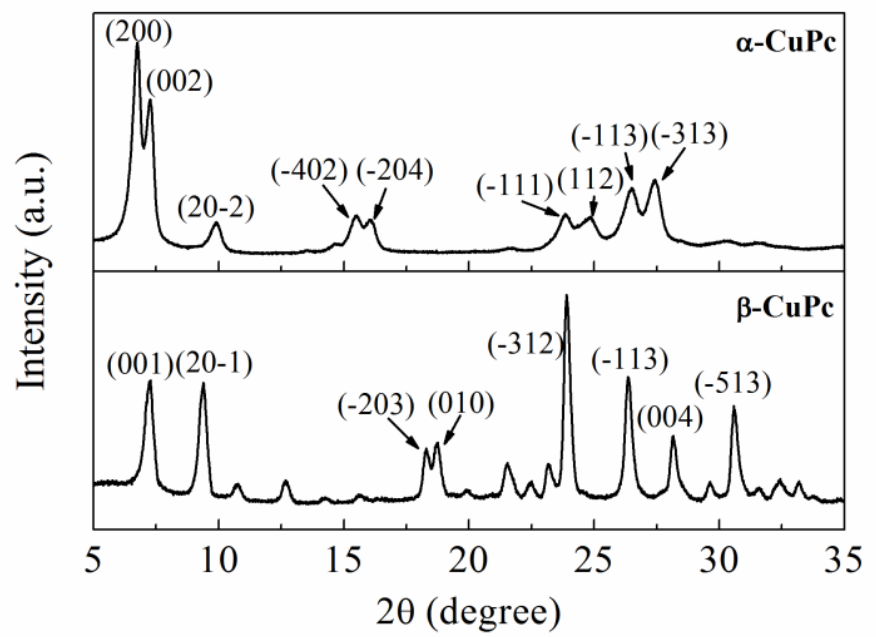

Fig. 4.2 XRD patterns of $\alpha$-CuPc and $\beta$-CuPc powder sample with Miller indices of major lines shown. No impurity lines were observed in either case. 


\subsubsection{Scanning Electron Microscopy (SEM)}

Scanning electron micrographs of both $\alpha-\mathrm{CuPc}$ and $\beta$-CuPc powder samples were obtained by using a JEOL 7600 FE-SEM system at the DOE National Energy Technology Laboratory (NETL). These micrographs shown in Fig. 4.3 were acquired by Mr. James Poston of NETL. It is evident from Fig. 4.3 (a) that $\alpha-\mathrm{CuPc}$ has particles of irregular shape with size of some particles as low as $30 \mathrm{~nm}$ where as $\beta-\mathrm{CuPc}$ has rod-like morphology (Fig. 4.3b).
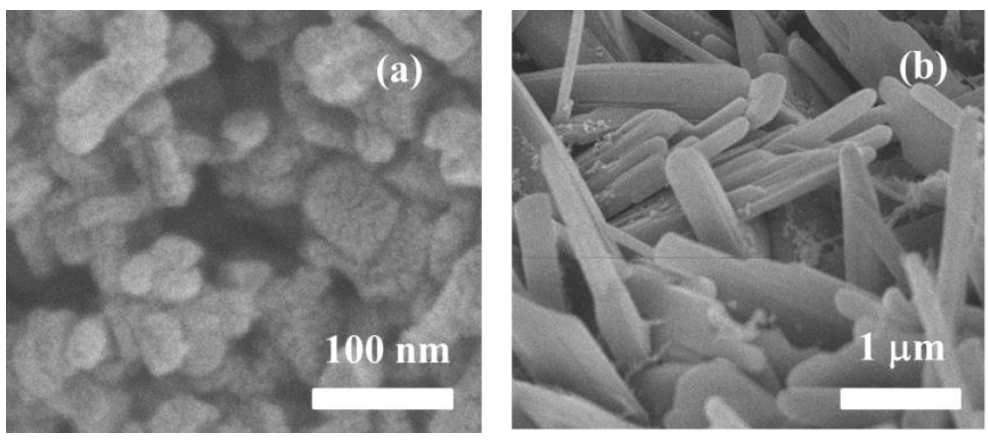

Fig. 4.3 Scanning electron micrographs for (a) $\alpha$-CuPc and (b) $\beta$-CuPc. The length scale bars are $100 \mathrm{~nm}$ and $1 \mu \mathrm{m}$ for $(a)$ and $(b)$, respectively.

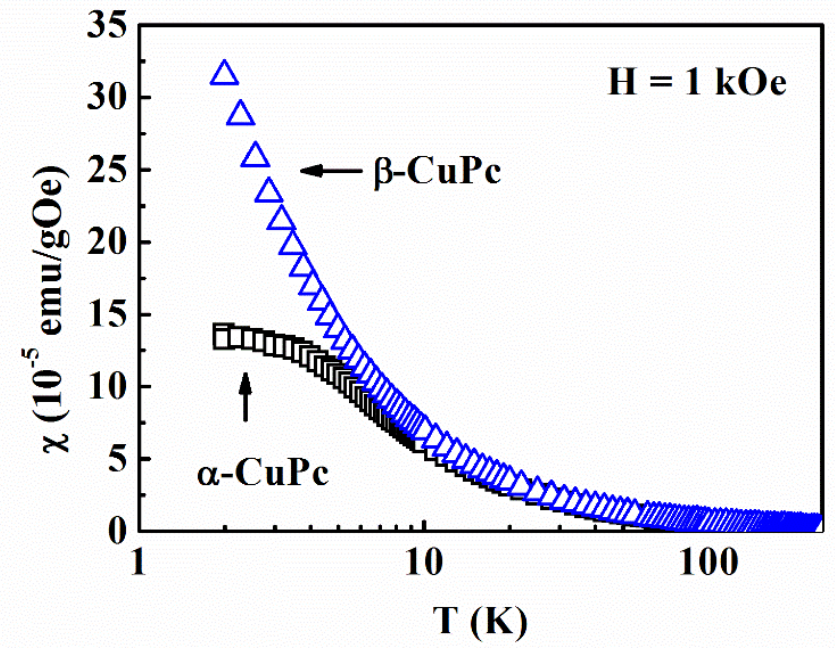

Fig. 4.4 Magnetic susceptibility $\chi$ vs. temperature T data for $\alpha$-CuPc and $\beta$-CuPc in $H=1 \mathrm{kOe}$. 


\subsection{Results from Magnetic Measurements, Analysis, and Discussion}

\subsubsection{Temperature Dependence of DC Magnetization and the Curie-Weiss Law}

Measurements of the magnetization $\mathrm{M}$ as a function of temperature $\mathrm{T}$ and magnetic field $\mathrm{H}$ were performed using the physical property measurement system (PPMS) described in Chapter 3. The temperature dependence of the magnetic susceptibility $\chi=(\mathrm{M} / \mathrm{H})$ for both $\alpha-\mathrm{CuPc}$ and $\beta$-CuPc samples measured at $\mathrm{H}=1 \mathrm{kOe}$ is shown in Fig. 4.4. For both samples, $\chi$ increases with decreasing T. However for $\alpha-\mathrm{CuPc}$, a plateau is reached around $2 \mathrm{~K}$ whereas it is absent for $\beta$-CuPc.

The data were first fit to the modified Curie-Weiss (CW) law, $\chi=\chi_{0}+\mathrm{C} /(\mathrm{T}-\theta)$, where $\chi_{\mathrm{o}}$ is the diamagnetic contribution estimated from the high-temperature limit by plotting $\chi$ vs. $1 / \mathrm{T}$ and extrapolating to the limit $1 / \mathrm{T}=0$ (see Fig. 4.5). The fits give $\chi_{0}=-2.96 \times 10^{-6}\left(-2.70 \times 10^{-6}\right.$ ) emu/gOe for $\alpha-\mathrm{CuPc}(\beta-\mathrm{CuPc})$. The diamagnetic contributions to $\chi_{0}$ come from the samples as well as the sample holder used in the measurements although the effects of $\chi_{\mathrm{o}}$ are only noticeable at $\mathrm{T}>$ about $100 \mathrm{~K}$. However this correction is essential for the accurate analysis of the data in the whole temperature range. 


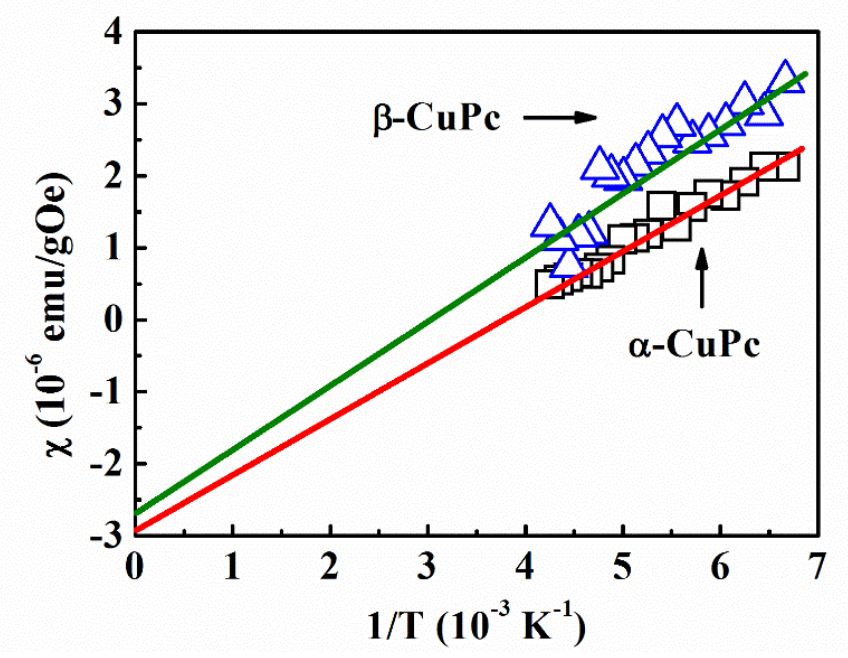

Fig. 4.5 Magnetic susceptibility $\chi$ vs. $1 /$ T plots for $\alpha-C u P c$ and $\beta$-CuPc. The solid lines are linear fits with the intercepts giving $\chi_{o}=-2.96 \times 10^{-6}\left(-2.70 \times 10^{-6}\right)$ emu/gOe for $\alpha-C u P c(\beta-C u P c)$.

After determining $\chi_{0}$, next $\left(\chi-\chi_{0}\right)^{-1}$ vs. T is plotted as shown in Fig. 4.6 to check the validity of the Curie-Weiss law. The linear fits in Fig. 4.6 yielded $\mathrm{C}=7.96 \times 10^{-4}\left(7.20 \times 10^{-4}\right)$ emu-K/gOe and $\theta=-$ $2.3 \mathrm{~K}(-0.2 \mathrm{~K})$ for $\alpha-\mathrm{CuPc}(\beta-\mathrm{CuPc})$, the negative $\theta$ signifying antiferromagnetic exchange coupling. Using the above magnitudes of $\chi_{0}, \mathrm{C}$, and $\theta$, fits to the modified $\mathrm{CW}$ law and experimental data are compared in Fig. 4.7 for both $\alpha-\mathrm{CuPc}$ and $\beta$-CuPc. From the solid lines in Fig. 4.7 representing fits to the $\mathrm{CW}$ law, it is evident that for $\alpha-\mathrm{CuPc}$, the data does not fit the $\mathrm{CW}$ law below about $4 \mathrm{~K}$ whereas for $\beta$-CuPc, the fit to the $\mathrm{CW}$ law is quite good down to $2 \mathrm{~K}$, the lowest temperature attainable in these experiments. 


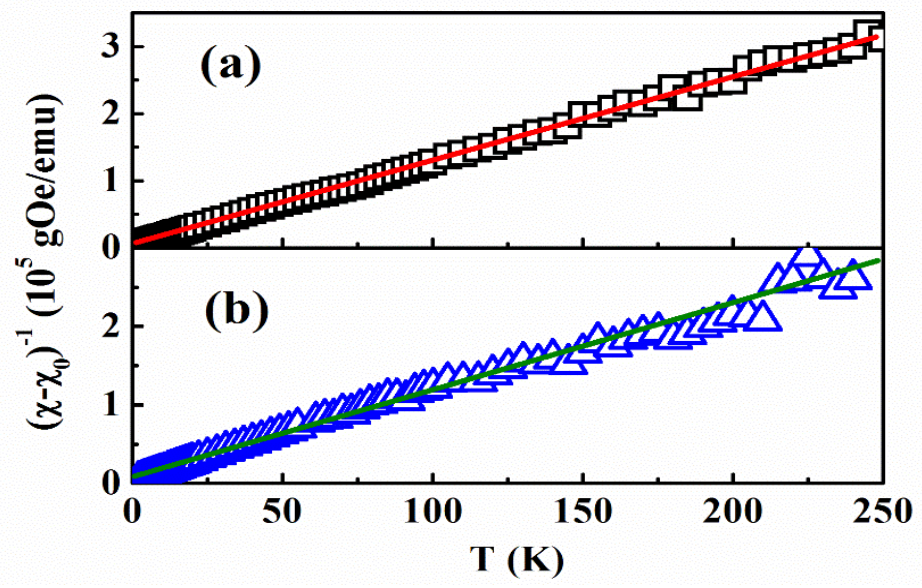

Fig. 4.6 Plots of $\left(\chi-\chi_{o}\right)^{-1} v$ s. Tfor (a) $\alpha-C u P c$ and (b) $\beta$-CuPc. The solid lines are linear fits representing the $C W$ law with $C=7.96 \times 10^{-4}\left(7.20 \times 10^{-4}\right)$ emu-K/gOe and $\theta=-2.3 \mathrm{~K}(-0.2 \mathrm{~K})$ for $\alpha-C u P c(\beta-C u P c)$.

As discussed in Chapter 2, the Curie constant $\mathrm{C}=\mathrm{N} \mu^{2} / 3 \mathrm{k}_{\mathrm{B}}$ where $\mathrm{N}$ is the number of magnetic ions per gm, $\mu$ is the magnetic moment per ion and $\mathrm{k}_{\mathrm{B}}$ is the Boltzmann constant. Using the evaluated $\mathrm{C}=7.96 \times 10^{-4} \mathrm{emu}-\mathrm{K} / \mathrm{gOe}$ for $\alpha-\mathrm{CuPc}$ yields magnetic moment $\mu=1.9145 \mu_{\mathrm{B}}$ which in turn yields $S=1 / 2$ and $g=2.21$ using $\mu^{2}=g^{2} S(S+1) \mu_{B}^{2}$, as predicted for $\mathrm{Cu}^{2+}$ in $\alpha$-CuPc by the DFT calculations of Mugarza et al. [9]. Also $\theta=-2.3 \mathrm{~K}$ shows antiferromagnetic (AFM) $\mathrm{Cu}^{2+}-\mathrm{Cu}^{2+}$ exchange coupling which is also evident by deviations from the $\mathrm{CW}$ law below $4 \mathrm{~K}$ in Fig. 4.7. Using the similar procedure for $\beta-\mathrm{CuPc}$ and the fitted $\mathrm{C}=7.20 \times 10^{-4} \mathrm{emu}-\mathrm{K} / \mathrm{gOe}$, yields $\mu=1.8221 \mu_{\mathrm{B}}$, spin $\mathrm{S}=1 / 2$ and $\mathrm{g}$ $=2.10$. The magnitude of $\theta=-0.2 \mathrm{~K}$ in $\beta-\mathrm{CuPc}$ shows a considerable weaker $\mathrm{AFM} \mathrm{Cu}^{2+} \mathrm{Cu}^{2+}$ exchange coupling in this system. Also, note that the g-factor for both $\alpha$-CuPc and $\beta$-CuPc shows the positive g-shift because the shell is more than half-filled, as discussed in Chapter 2. These g-shifts result from the partially restored orbital contributions to the magnetic moment via the spin-orbit interaction, as shown in Eq. (2.35) and Eq. (2.37). 


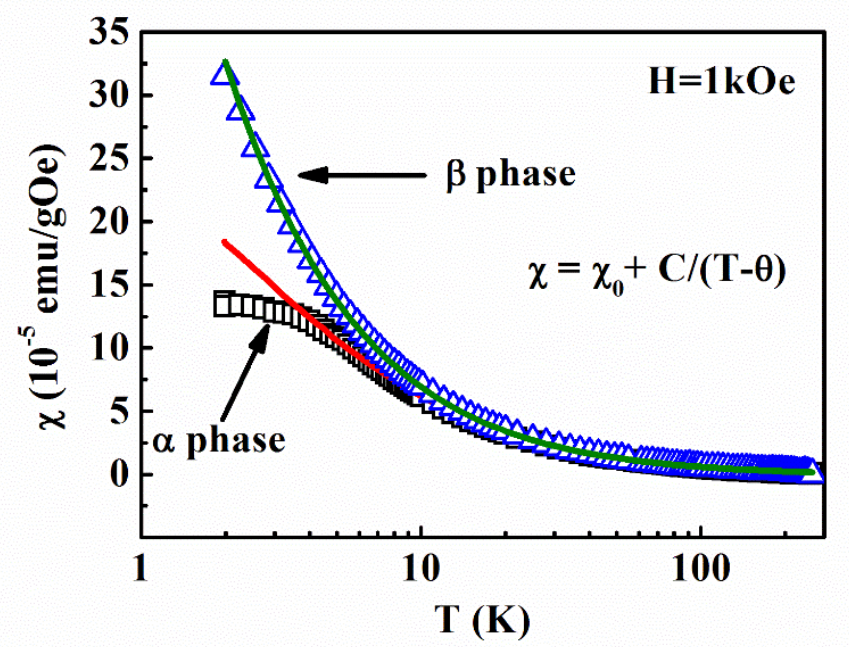

Fig. 4.7 Magnetic susceptibility $\chi$ vs. temperature Tfor $\alpha-C u P c$ and $\beta$-CuPc. The solid lines are fits to the modified Curie-Weiss law with the fitting parameters listed in the caption to Fig. 4.6.

The Heisenberg exchange constant $\mathrm{J}_{\mathrm{ex}} / \mathrm{k}_{\mathrm{B}}$ can be estimated by the mean field theory following Eq.

(4.1) derived in Chapter 2:

$$
\mathrm{J}_{\mathrm{ex}}=\frac{3 \theta}{2 \mathrm{ZS}(\mathrm{S}+1)}
$$

Here $\theta$ is the Weiss constant, $\mathrm{S}$ is the spin, and $\mathrm{Z}$ is the number of the nearest neighbors of each $\mathrm{Cu}^{2+}$. For both $\alpha-\mathrm{CuPc}$ and $\beta-\mathrm{CuPc}, \mathrm{Z}=2$ (for a linear chain) and spin $\mathrm{S}=1 / 2$ yielding

$$
\mathrm{J}_{\mathrm{ex}}=\theta
$$

This gives exchange constant $\mathrm{J}_{\mathrm{ex}} / \mathrm{k}_{\mathrm{B}}=-2.3 \mathrm{~K}(-0.2 \mathrm{~K})$ for $\alpha-\mathrm{CuPc}(\beta-\mathrm{CuPc})$ using the mean-field theory. These magnitudes of $\mathrm{J}_{\mathrm{ex}} / \mathrm{k}_{\mathrm{B}}$ are compared with those obtained from fitting the $\chi$ vs. temperature $\mathrm{T}$ data to the more accurate Bonner-Fisher model. This analysis and discussion is presented in the following section. 


\subsubsection{Fit to the Bonner-Fisher Model}

The chain configuration of the CuPc molecules along the b-axis is evident from Fig. 1.1. Consequently the data of $\chi$ vs. T was next fitted to the predictions of the Bonner-Fisher (BF) model [10] for a spin $S=1 / 2$ linear chain with Heisenberg AFM exchange-coupling $\mathrm{J}_{\mathrm{ex}}$ defined by the Hamiltonian $\mathrm{H}$ $=-2 \mathrm{~J}_{\mathrm{ex}} \Sigma \mathbf{S}_{\mathbf{i}} \cdot \mathbf{S}_{\mathbf{i}+\mathbf{1}}$. Following Eggert et al. [11] and Feyerherm et al. [12], the variation of $\chi$ vs. T for a linear chain model $S=1 / 2$ system with the normalized AFM coupling parameter $x=\left|J_{\text {ex }}\right| /\left(k_{B} T\right)$ is given by

$$
\begin{gathered}
\chi=\chi_{0}+\frac{C}{T} F(x) \\
F(x)=\frac{1+a_{1} x+a_{2} x^{2}}{1+b_{1} x+b_{2} x^{2}+b_{3} x^{3}}
\end{gathered}
$$

Here $\mathrm{C}$ is the Curie constant, $\mathrm{a}_{1}=0.17032, \mathrm{a}_{2}=0.93404, \mathrm{~b}_{1}=1.46764, \mathrm{~b}_{2}=0.54784$, and $\mathrm{b}_{3}=4.28544$.

Note that for $\mathrm{x}=0$ (meaning $\left.\mathrm{J}_{\mathrm{ex}}=0\right), \chi=\mathrm{C} / \mathrm{T}$ is obtained which is the usual Curie-law as expected. In Fig. 4.8 , the fit of the data of $\chi$ vs. T to the predictions of this model for both $\alpha-\mathrm{CuPc}$ and $\beta$-CuPc are shown as solid lines with $\mathrm{J}_{\mathrm{ex}} / \mathrm{k}_{\mathrm{B}}=-1.7 \mathrm{~K}(-0.2 \mathrm{~K})$ for $\alpha-\mathrm{CuPc}(\beta-\mathrm{CuPc})$, within uncertainty of $0.1 \mathrm{~K}$ in the magnitudes of $\mathrm{J}_{\mathrm{ex}} / \mathrm{k}_{\mathrm{B}}$. In these fits, the magnitude of $\mathrm{J}_{\mathrm{ex}}$ was varied till the best fit was obtained in the whole temperature range. Excellent fits are obtained in both cases with the model correctly showing the broad maximum indicated in the $\chi$ vs. T data for $\alpha$-CuPc. For $\beta$-CuPc, the broad maximum is predicted to occur near $\mathrm{T}=0.25 \mathrm{~K}$ (inset of Fig. 4.8) because of the lower magnitude of $\mathrm{J}_{\mathrm{ex}} / \mathrm{k}_{\mathrm{B}}=-0.2 \mathrm{~K}$ in this system. However this lower temperature of $\mathrm{T}=0.25 \mathrm{~K}$ was not attainable in our experiments.

The CW law can also be derived from Eq. (4.4) by retaining only the first order term in $\mathrm{x}$. This 
exercise leads to the CW law with $\theta=1.29732 \mathrm{~J}_{\mathrm{ex}} / \mathrm{k}_{\mathrm{B}}$. Using $\mathrm{J}_{\mathrm{ex}} / \mathrm{k}_{\mathrm{B}}=-1.7 \mathrm{~K}(-0.2 \mathrm{~K})$ for $\alpha-\mathrm{CuPc}(\beta-\mathrm{CuPc})$ yields $\theta=-2.2 \mathrm{~K}(\theta=-0.26 \mathrm{~K})$ for $\alpha-\mathrm{CuPc}(\beta-\mathrm{CuPc})$, comparing favorably with the experimental values of $\theta=-2.3 \mathrm{~K}$ and $\theta=-0.2 \mathrm{~K}$ respectively. At lower temperatures, higher order terms in $\mathrm{x}$ become increasingly important particularly for linear chains and 2-D systems [14].

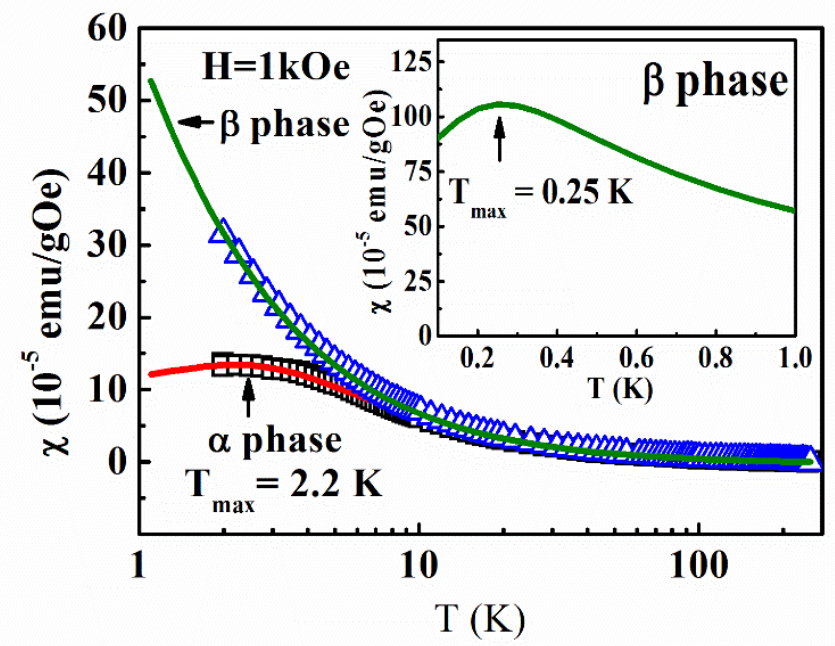

Fig. 4.8 Data of $\chi$ vs. T of Fig. 3.5 for the $\alpha$-and $\beta$-phases of CuPc are fitted to Eq. (3.1) with the solid lines as fits yielding the exchange constant $J_{e x} / k_{B}=-1.7 \mathrm{~K}(-0.2 \mathrm{~K})$ for $\alpha(\beta)$ phase. The fit also gives $T_{\max }$ $=2.2 \mathrm{~K}$ for $\alpha$-phase and calculated $T_{\max }=0.25 \mathrm{~K}$ for $\beta$-phase (see inset).

Since the $\mathrm{Cu}^{2+}-\mathrm{Cu}^{2+}$ exchange interaction requires overlap of the wave functions of neighboring $\mathrm{Cu}^{2+}$ ions either directly or through the intervening $\mathrm{N}$ atoms, this order of magnitude difference in the magnitude of $\mathrm{J}_{\mathrm{ex}} / \mathrm{k}_{\mathrm{B}}$ is likely due to the smaller $\mathrm{Cu}-\mathrm{Cu}$ distance in the $\alpha$-phase than in $\beta$-phase (Fig. 1.1) as noted earlier. The difference in the stack angles $\delta$ of the two phases may also be an important factor (see Fig. 1.1). The recent calculations of Wu et al. [13] using DFT have predicted $\mathrm{J}_{\mathrm{ex}} / \mathrm{k}_{\mathrm{B}}=$ - $1.3 \mathrm{~K}(-0.14 \mathrm{~K})$ for $\alpha-\mathrm{CuPc}(\beta-\mathrm{CuPc})$. Our experimentally measured values of $\mathrm{J}_{\mathrm{ex}} / \mathrm{k}_{\mathrm{B}}$ for both systems are about $30 \%$ larger than the DFT predictions. For $\mathrm{S}=1 / 2$, the temperature $\mathrm{T}_{\max }$ where the maximum in 
$\chi$ vs. $\mathrm{T}$ occurs is given by $\mathrm{T}_{\max } \simeq 1.3 \mid \mathrm{J}_{\mathrm{ex}} / \mathrm{k}_{\mathrm{B}}$ for the linear chain Bonner-Fisher model whereas $\mathrm{T}_{\max } \simeq$ $0.91 \mathrm{~J}_{\mathrm{ex}} / \mathrm{k}_{\mathrm{B}}$ for 2-D square lattice [14].

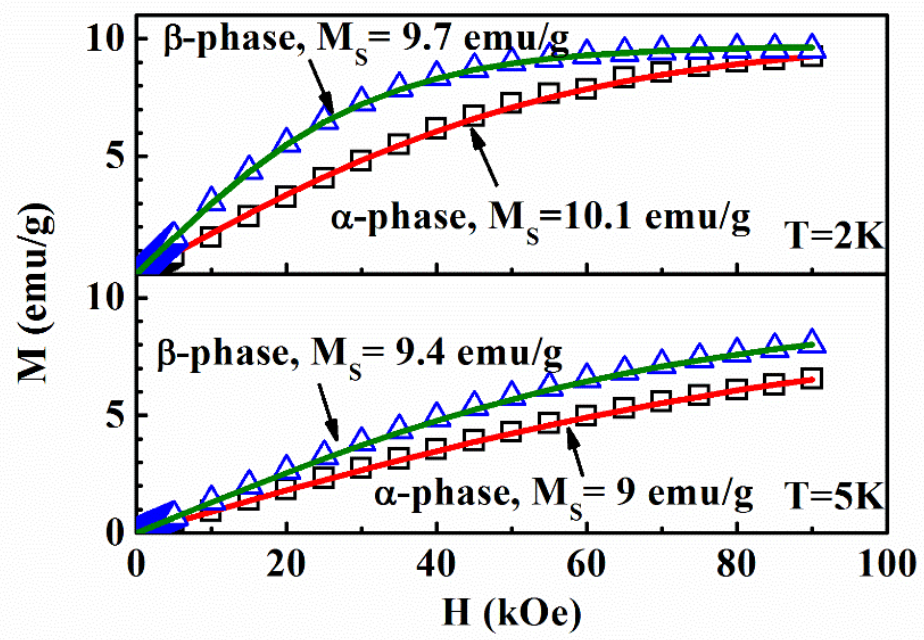

Fig. 4.9 Magnetization $M$ vs. magnetic field $H$ of $\alpha$ - and $\beta$-phases of $C u P c$ at $2 K$ and $5 \mathrm{~K}$. The solid lines represent fits to Eq. (4.5) with $\theta=-2.3 \mathrm{~K}(-0.2 \mathrm{~K})$ for $\alpha(\beta)$-phase.

\subsubsection{Magnetic Field Dependence of Magnetization}

The isothermal data of $\mathrm{M}$ vs. $\mathrm{H}$ up to $\mathrm{H}=90 \mathrm{kOe}$ and measured at $2 \mathrm{~K}$ and $5 \mathrm{~K}$ for both samples are shown in Fig. 4.9. In the absence of exchange interaction $(\theta=0)$, the data of $\mathrm{M}$ vs. $\mathrm{H}$ are expected to fit the Brillouin function variation which for $S=1 / 2$ reduces to the following Eq. (4.5) in which the effect of exchange interaction is taken into account by including $\theta$ analogous to the CW law [15]:

$$
M=M_{S} \tanh \frac{g \mu_{B} H}{2 k_{B}(T-\theta)}
$$


Efforts to fit the data with $\theta=0$ led to unsatisfactory fits especially for $\alpha$-CuPc because of the larger magnitude of $\theta$ and hence exchange interaction in this system. Next, the data is fitted with $\theta=-2.3$ $\mathrm{K}$ ( $\alpha$-phase) and $\theta=-0.2 \mathrm{~K}(\beta$-phase) in analogy with the CW law noted earlier, yielding good fits to the data with $\mathrm{M}_{\mathrm{S}}=10.1 \mathrm{emu} / \mathrm{g}(9 \mathrm{emu} / \mathrm{g})$ at $2 \mathrm{~K}(5 \mathrm{~K})$ for $\alpha-\mathrm{CuPc}$ and $\mathrm{M}_{\mathrm{S}}=9.7 \mathrm{emu} / \mathrm{g}(9.4 \mathrm{emu} / \mathrm{g})$ at $2 \mathrm{~K}(5$ K) for $\beta$-CuPc. The calculated magnitude of $\mathrm{M}_{\mathrm{S}}=\mathrm{Ng} \mu_{\mathrm{B}} \mathrm{S}=10.7 \mathrm{emu} / \mathrm{g}$ for $\alpha-\mathrm{CuPc}$ and $\mathrm{M}_{\mathrm{S}}=10.2 \mathrm{emu} / \mathrm{g}$ for $\beta$-CuPc at $0 \mathrm{~K}$ using $\mathrm{S}=1 / 2$ and $\mathrm{g}=2.21$ (2.10) evaluated earlier for $\alpha$-CuPc $(\beta-\mathrm{CuPc})$. This discrepancy of few percent between the calculated and experimental magnitudes of $\mathrm{M}_{\mathrm{S}}$ likely results from lack of saturation at $2 \mathrm{~K}$ and from the effects of zero-point spin deviations known to exist in antiferromagnetically coupled systems [16].

\subsubsection{Temperature Dependence of AC Magnetization}

The AC magnetization for $\alpha-\mathrm{CuPc}$ was measured from $2 \mathrm{~K}$ to $20 \mathrm{~K}$ without DC magnetic field but with a small $\mathrm{AC}$ magnetic field $\mathrm{H}_{\mathrm{ac}}=5$ Oe. As shown in Fig. 4.10, the real part of susceptibility $\chi^{\prime}$ is the same for all three frequencies. For the imaginary part of susceptibility $\chi^{\prime \prime}$, there is a hint of weak peaks near $2.5 \mathrm{~K}$. However, the data are too noisy because of the smaller magnitudes of $\chi^{\prime \prime}$ and so no reliable result for the shift of the peak with the measuring frequency could be obtained which is essential for determining the specifics of the relaxation phenomenon. Such an analysis is presented in the case of $\beta-\mathrm{MnPc}$ in a later chapter. 


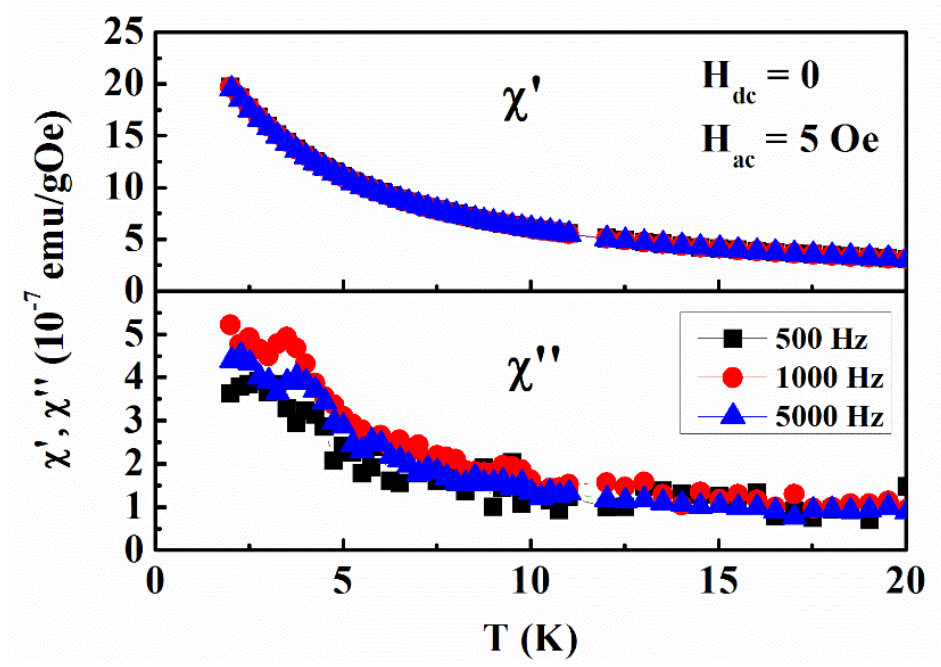

Fig. 4.10 Temperature dependence of the ac susceptibilities $\chi^{\prime}$ and $\chi^{\prime \prime}$ for $\alpha$-CuPc measured in zero applied static field with $\mathrm{H}_{a c}=5$ Oe at frequency $f=500 \mathrm{~Hz}, 1000 \mathrm{~Hz}$, and $5000 \mathrm{~Hz}$.

\subsection{Conclusions:}

The major conclusions from the results presented in this chapter can be summarized as follows: (i)

The electronic state of $\mathrm{Cu}$ in both $\alpha-\mathrm{CuPc}$ and $\beta-\mathrm{CuPc}$ is shown to be $\mathrm{Cu}^{2+}$ with spin $1 / 2$; (ii) The fits of the magnetic susceptibility versus temperature data to the Curie-Weiss law yields $\theta=-2.3 \mathrm{~K}, \mu=$ $1.9145 \mu_{\mathrm{B}}$, and $\mathrm{g}=2.21$ for spin $\mathrm{S}=1 / 2$ for $\alpha-\mathrm{CuPc}$; and $\theta=-0.2 \mathrm{~K}, \mu=1.822 \mu_{\mathrm{B}}, \mathrm{g}=2.10$ for spin $\mathrm{S}=$ $1 / 2$ for $\beta$-CuPc. The g-shifts from the free electron value of 2.0023 result from the partially restored orbital contribution to the magnetic moment via spin-orbit coupling; (iii) The fits of the magnetic susceptibility versus temperature data to the Bonner-Fisher model for linear Heisenberg chains yield the Heisenberg exchange constant $\mathrm{J}_{\mathrm{ex}} / \mathrm{k}_{\mathrm{B}}=-1.7 \mathrm{~K}(-0.2 \mathrm{~K})$ between the $\mathrm{Cu}^{2+}-\mathrm{Cu}^{2+}$ ions in $\alpha-\mathrm{CuPc}(\beta-\mathrm{CuPc})$. This order of magnitude difference in the magnitude of $\mathrm{J}_{\mathrm{ex}} / \mathrm{k}_{\mathrm{B}}$ is likely due to the smaller $\mathrm{Cu}-\mathrm{Cu}$ distance in the $\alpha$-phase than in $\beta$-phase; (iv) The isothermal data of $\mathrm{M}$ vs. $\mathrm{H}$ at $2 \mathrm{~K}$ and $5 \mathrm{~K}$ are interpreted by including the effect of the exchange interaction; (v) Finally, the results show that both $\alpha$ - and $\beta$-CuPc are 
good examples of antiferromagnetically coupled Heisenberg linear chains with spin $S=1 / 2$ and negligible interchain coupling.

\section{References}

[1] S. Heutz, C. Mitra, W. Wu, A.J. Fisher, A. Kerridge, M. Stoneham, A.H. Harker, J. Gardener, H.-H.

Tseng, T.S. Jones, C. Renner and G. Aeppli, Adv. Mater. 19, 3618 (2007).

[2] Z. Wang, K.L. Pisane and M.S. Seehra, IEEE. Trans. Mag. 51, 2700104 (2015).

[3] L. Lozzi, S. Santucci, R. La Rosa, B. Delley and S. Picozzi, J. Chem. Phys. 121, 1883 (2004).

[4] A. Hoshino, Y. Takenaka and H. Miyaji, Acta. Crystallogr. B. 59, 393 (2003).

[5] E. Jungyoon, S. Kim, E. Lim, D. Cha and B. Friedman, Appl. Surf. Sci. 205, 274 (2003).

[6] O. Berger, W.-J. Fischer, B. Adolphi, S. Tierbach, V. Melev and J. Schreiber, J. Mater. Sci., Mater.

Electron. 11, 331 (2000).

[7] C.J. Brown, J. Chem. Soc. A. 90, 2488 (1968).

[8] W. Wu, A. Kerridge, A.H. Harker and A.J. Fisher, Phys. Rev. B. 77, 184403 (2008). 
[9] A. Mugarza, R. Robles, C. Krull, R. Korytar, N. Lorente and P. Gambardella, Phys. Rev. B. 85, 155437 (2012).

[10] J.C. Bonner and M.E. Fisher, Phys. Rev. 135, A640 (1964).

[11] S. Eggert, I. Affleck and M. Takahashi, Phys. Rev. Lett. 73, 332 (1994).

[12] R. Feyerhem, S. Abens, D. Gunther, T. Ishida, M. Meibner, M. Meschke, T. Nogami and M. Steiner, J. Phys. Condens. Matter. 12, 8495 (2000).

[13] W. Wu, A.J. Fisher and N.M. Harrison, Phys. Rev. B. 84, 024427 (2011).

[14] M.S. Seehra, Phys. Lett. A. 28, 754 (1969).

[15] V. Narang, D. Korakakis and M.S. Seehra, J. Appl. Phys. 116, 213911 (2014).

[16] P.W. Anderson, Phys. Rev. 86, 694 (1952). 


\section{CHAPTER 5.}

\section{Magnetic Properties of $\beta$-Cobalt Phthalocyanine}

\subsection{Introduction}

In this chapter, results from the measurement of the magnetization (M) of $\beta$-CoPc are presented covering the temperature range of $0.4 \mathrm{~K}$ to $300 \mathrm{~K}$ and in magnetic fields $\mathrm{H}$ up to $90 \mathrm{kOe}$. The data are analyzed and discussed in terms of Curie-Weiss (CW) law and the Bonner-Fisher (BF) model for a Heisenberg linear chain antiferromagnetim (HLCA) with spin $S=1 / 2$. The intrachain exchange constant $\mathrm{J}_{\mathrm{ex}} / \mathrm{k}_{\mathrm{B}}=-2 \mathrm{~K}$ for $\beta$-CoPc was reported using DFT (density functional theory) calculation by Wu et al. [1]. Also, in a recent reported magnetic studies of $\beta$-CoPc [2], the intrachain exchange constant $\mathrm{J}_{\mathrm{ex}} / \mathrm{k}_{\mathrm{B}}=-1.9$ $\mathrm{K}$ was determined experimentally from the fit of the magnetization $\mathrm{M}$ vs. magnetic field $(\mathrm{H})$ data at $2 \mathrm{~K}$ to the predictions of the BF model. However, since the reported $\chi$ vs. $\mathrm{T}$ data were limited to temperatures $\mathrm{T}>8 \mathrm{~K}$, the observation of $\mathrm{T}_{\max }$ characteristic of an HLCA was not observed in $\beta$-CoPc [2]. Thus, in this chapter, the observation of $\mathrm{T}_{\max }$ at $1.9 \mathrm{~K}$ and fits of $\chi \mathrm{vs}$. $\mathrm{T}$ data and the isothermal $\mathrm{M}$ vs. $\mathrm{H}$ data at $1 \mathrm{~K}$ to the Bonner-Fisher model are the important new results. A major portion of the results presented in this chapter have been published by the author in a recent paper [3].

\subsection{Sample and its Structural Characterization}

\subsubsection{Sample}

The powder sample of CoPc used here was purchased from Alfa Aesar (stock \# 41496) and used as received. X-ray diffraction (XRD) analysis confirmed this powder sample as $\beta$-phase (shown later). An 
attempt was made to prepare $\alpha$-phase $\mathrm{CoPc}$ from this $\beta$-phase $\mathrm{CoPc}$ using the acid pasting method described in literature [4]. However this attempt was not successful. So the results presented here are only for the $\beta$-CoPc powder sample.

\subsubsection{X-ray Diffraction (XRD)}

X-ray diffraction (XRD) analysis of the purchased sample was performed at room temperature using a PANalytical X'Pert Pro X-ray diffractometer with $\mathrm{Cu}-\mathrm{K} \alpha$ source $(\lambda=0.15418 \mathrm{~nm})$. The XRD pattern of the sample shown in Fig. 5.1 agrees with the expected pattern of $\beta$-CoPc (ICDD \#02-061-3631) with space group $\mathrm{P} 2{ }_{1} / \mathrm{c}$ and two molecules per unit cell in the monoclinic structure with lattice parameters: $\mathrm{a}=1.4542 \mathrm{~nm}, \mathrm{~b}=0.4773 \mathrm{~nm}, \mathrm{c}=1.9352 \mathrm{~nm}$, and $\beta=120.82^{\circ}[5]$. This structure is shown in Chapter 1 ,

Fig. 1.1(d). Note that all the observed peaks in the XRD pattern match well with the ICDD pattern for $\beta$-CoPc. The lower observed intensity of the (100) peak is likely due to the plate-like preferred orientation of the sample shown below by scanning electron microscopy.

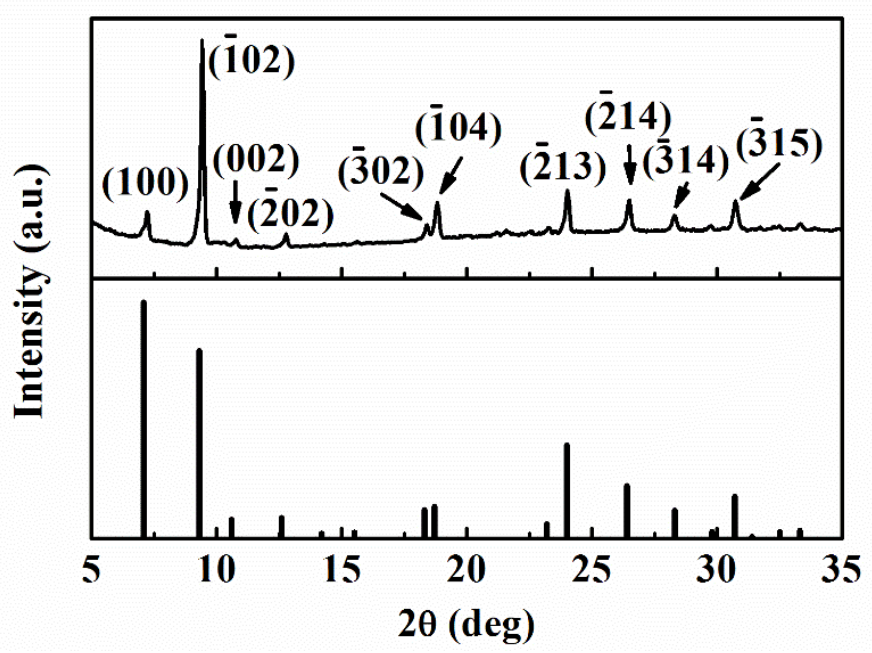

Fig. 5.1 The top panel shows the XRD pattern of $\beta$-CoPc sample with Miller indices of major lines marked; the bottom panel shows the ICDD data of $\beta$-CoPc. 


\subsubsection{Scanning Electron Microscopy (SEM)}

The SEM of the $\beta$-CoPc powder sample was done using a JEOL 7600 FE-SEM system at the DOE National Energy Technology Laboratory and it shows the plate-like morphology (Fig. 5.2).

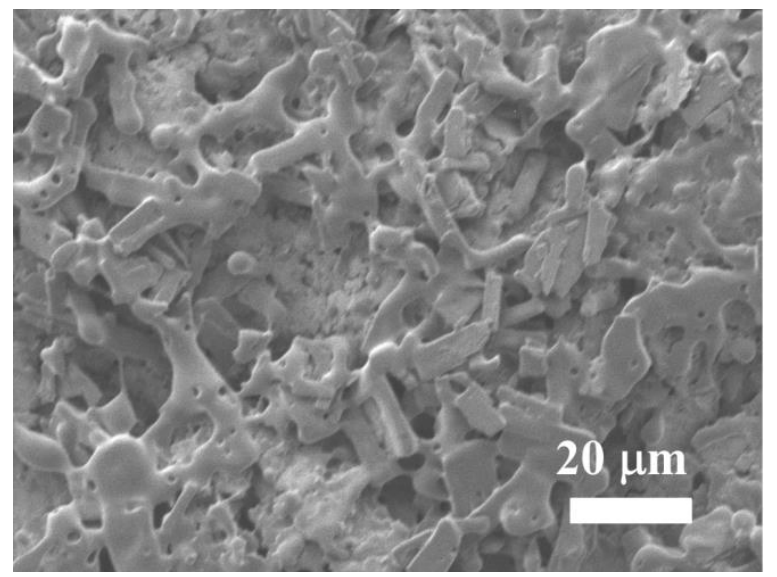

Fig. 5.2 A Scanning electron micrograph for the $\beta$-CoPc powder sample.

\subsection{Results from Magnetic Measurements, Analysis, and Discussion}

\subsubsection{Temperature Dependence of DC Magnetization and the Curie-Weiss Law}

Measurements of the magnetization (M) of the $\beta$-CoPc powder sample from $2 \mathrm{~K}$ to $300 \mathrm{~K}$ and in magnetic fields up to $90 \mathrm{kOe}$ were carried out using the in-house Physical Property Measurements System (PPMS) described in Chapter 3. In addition, the temperature dependence of the magnetization from $0.4 \mathrm{~K}$ to $20 \mathrm{~K}$ was also measured using the Superconducting Magnet 2 (SCM2) system at the National High Magnetic Field Laboratory (NHMFL) in Tallahassee, Florida.

At the NHMFL, the M vs. T data were obtained using a home-made susceptometer from $0.4 \mathrm{~K}$ to $20 \mathrm{~K}$ with applied DC field $\mathrm{H}=10 \mathrm{kOe}$, frequency $=331 \mathrm{~Hz}$, and ac field $\mathrm{H}_{\mathrm{ac}}=3.3$ Oe using the SCM2 
facility. The ac susceptibility data acquired at the NHMFL was normalized to the DC susceptibility data acquired using the PPMS at a single temperature (20 K). This can be justified because (i) ac susceptibility was found to be frequency independence; and (ii) the temperature dependence is practically identical between the data obtained by the two techniques. In general, if the magnetic relaxation is much faster than the measurement time scale (1 - $10 \mathrm{~ms}$ in this case), the ac susceptibility resembles the DC susceptibility, which seems to be the case for $\beta-\mathrm{CoPc}$ at least in the measured temperature range.

The temperature dependence of magnetic susceptibility $\chi$ measured with applied $\mathrm{H}=10 \mathrm{kOe}$ is shown in Fig. 5.3. Since M vs. H data is quite linear in $\mathrm{H}$ up to about $20 \mathrm{kOe}$ even at $2 \mathrm{~K}$ (see inset of Fig. 5.3), $\chi=M / H$ represents the differential susceptibility. Again, the data acquired at the NHMFL from 0.4 $\mathrm{K}$ to $20 \mathrm{~K}$ is merged with the data acquired with the PPMS from $2 \mathrm{~K}$ to $300 \mathrm{~K}$ by normalizing the former with the latter at $20 \mathrm{~K}$. The combined data shows a distinct peak at $\mathrm{T}_{\max }=1.9 \mathrm{~K}$, which is independent of frequency.

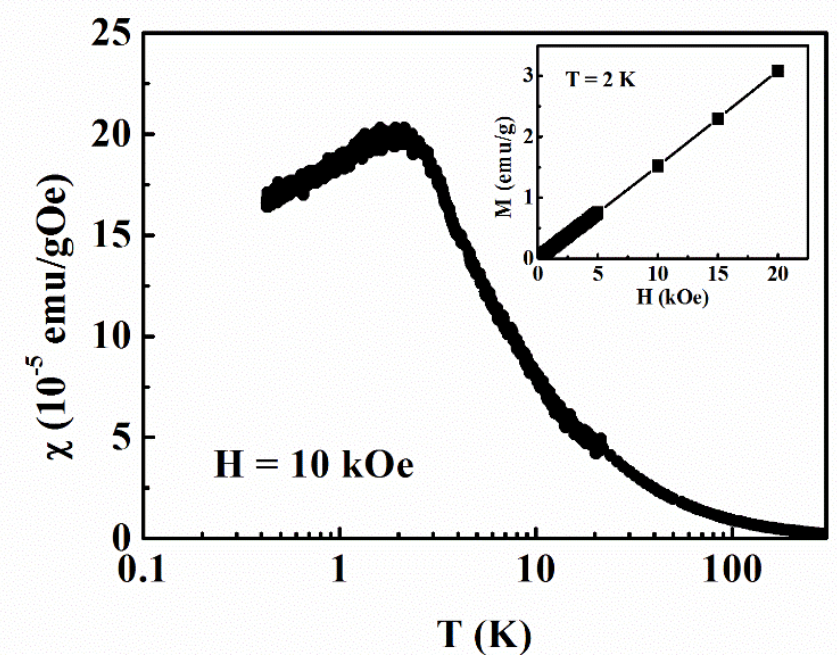

Fig. 5.3 Magnetic susceptibility $\chi$ vs. temperature Tof $\beta$-CoPc. The inset shows the Mvs. H. plot. 


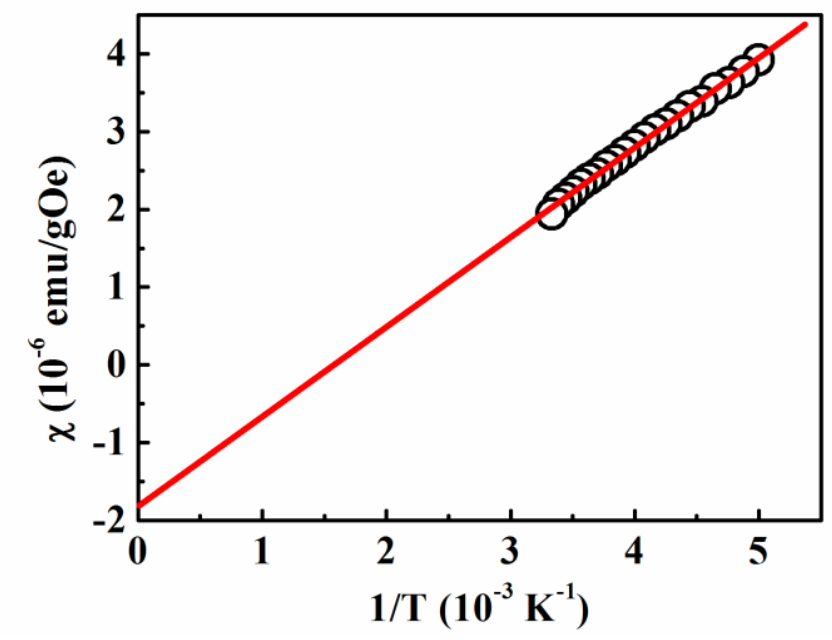

Fig. 5.4 Plot of magnetic susceptibility $\chi$ vs. $1 /$ T for $\beta$-CoPc with $H=10 \mathrm{kOe}$. The solid line is the linear fit of the experimental data and its intercept with $y$ axis $(\chi)$ gives $\chi_{o}=-1.8 \times 10^{-6} \mathrm{emu} / \mathrm{gOe}$.

The analysis of the data was done as follows. First the data acquired from the PPMS from $2 \mathrm{~K}$ to $300 \mathrm{~K}$ was fitted to the modified Curie-Weiss $(\mathrm{CW})$ law: $\chi=\chi_{0}+\mathrm{C} /(\mathrm{T}-\theta)$. For this purpose, $\chi_{0}=-1.8 \times 10^{-6}$ emu/gOe was determined by plotting $\chi$ vs. $1 / \mathrm{T}$ and linearly extrapolating the high temperature data to $1 / \mathrm{T}$ $\rightarrow 0$, as shown in Fig. 5.4. This $\chi_{0}$ includes the diamagnetic contributions of the plastic sample holder and the sample itself. However the effect of $\chi_{\mathrm{o}}$ is only noticeable for $\mathrm{T}>100 \mathrm{~K}$. Next, by plotting $\left(\chi-\chi_{0}\right)^{-1}$ vs. T (see Fig. 5.5), the linear fit yielded $\mathrm{C}=1.02 \times 10^{-3} \mathrm{emu}-\mathrm{K} / \mathrm{gOe}$ and $\theta=-2.5 \mathrm{~K}$, the negative $\theta$ signifying antiferromagnetic exchange coupling. In Fig. 5.6, the solid line represents the fit to the modified CW law with the magnitudes of $\chi_{0}, \mathrm{C}$, and $\theta$ listed above. From Fig. 5.6, it is evident that the data deviates from the $\mathrm{CW}$ fit below about $3 \mathrm{~K}$. 


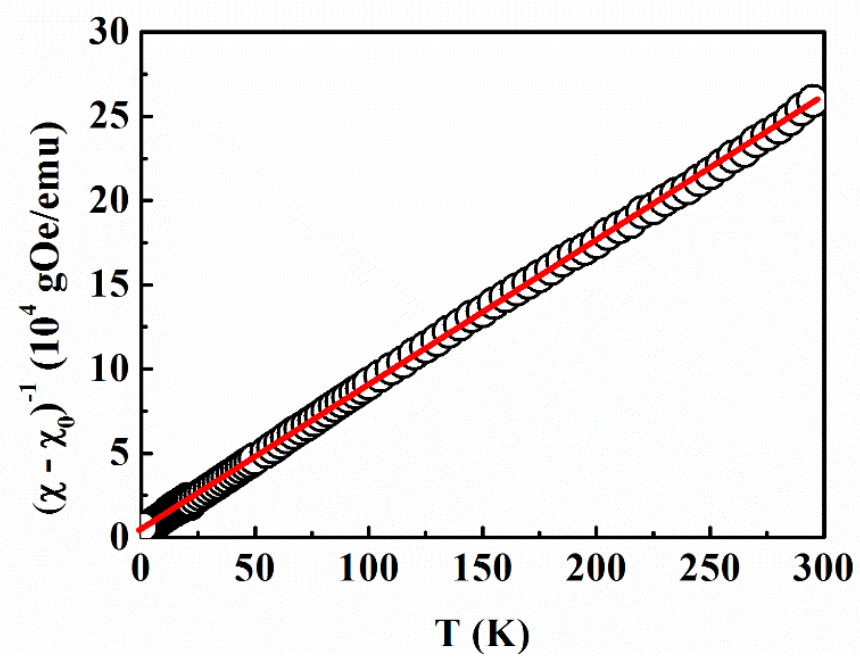

Fig. 5.5 Plot of the temperature dependence of the inverse magnetic susceptibility $\left(\chi-\chi_{o}\right)^{-1}$ of $\beta$-CoPc in $H=10 \mathrm{kOe}$. The solid line is the linear fit representing CW law.

Using $\mathrm{C}=\mathrm{N} \mu^{2} / 3 \mathrm{k}_{\mathrm{B}}$ where $\mathrm{N}$ is the number of magnetic ions per gm, each with magnetic moment $\mu$ and $\mathrm{k}_{\mathrm{B}}$ is the Boltzmann constant yields $\mu=2.16 \mu_{\mathrm{B}}$ per $\mathrm{Co}^{2+}$ ion. If the distribution of electrons for the $3 \mathrm{~d}^{7}$ sate of $\mathrm{Co}^{2+}$ obeyed the Hunds' rules, $\mu$ close to $4.8 \mu_{\mathrm{B}}$ would have been observed corresponding to spin $\mathrm{S}=3 / 2$ (see Table 1.2 ). Thus the observed $\mu=2.16 \mu_{\mathrm{B}}$ per $\mathrm{Co}^{2+}$ ion can only be explained if $S=1 / 2$. Using $\mu^{2}=g^{2} S(S+1)$ yields $g=2.49$ for the $S=1 / 2$ low-spin state of $\mathrm{Co}^{2+}$ in $\beta$-CoPc. Also, $\mathrm{g}=2.49$ rather than 2.00 implies some contribution from the partially restored orbital moment via spin-orbit coupling as in CuPc. The g-shift is positive since for $3 \mathrm{~d}^{7}$ state of $\mathrm{Co}^{2+}$, the shell is more than half-full as discussed in Chapter 2. 


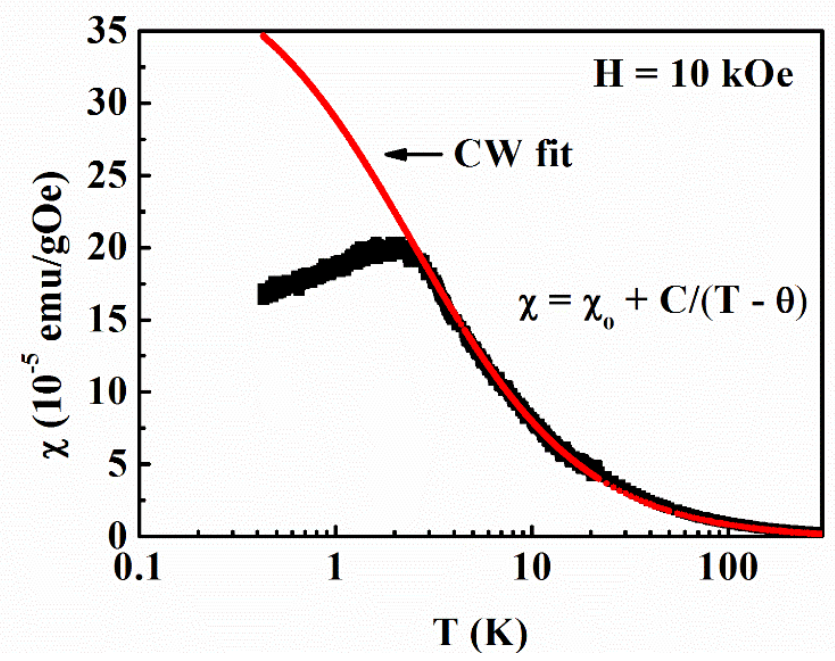

Fig. 5.6 Magnetic susceptibility $\chi$ vs. temperature Tof $\beta$-CoPc with $H=10 \mathrm{kOe}$. The Solid line is fit to the modified Curie-Weiss law: $\chi=\chi_{o}+C /(T-\theta)$ with the parameters: $\chi_{o}=-1.8 \times 10^{-6}$ emu/gOe, $C=1.02 \times$ $10^{-3} \mathrm{emu}-\mathrm{K} / \mathrm{gOe}$, and $\theta=-2.5 \mathrm{~K}$.

The Heisenberg exchange constant $\mathrm{J}_{\mathrm{ex}} / \mathrm{k}_{\mathrm{B}}$ can be estimated by the mean-field theory following:

$$
\mathrm{J}_{\mathrm{ex}}=\frac{3 \theta}{2 \mathrm{ZS}(\mathrm{S}+1)}
$$

Here $\theta$ is the Weiss constant, $\mathrm{S}$ is the spin, and $\mathrm{Z}$ is the number of the nearest neighbors of each $\mathrm{Co}^{2+}$. For $\beta$-CoPc, $Z=2$ (for a linear chain) and spin $S=1 / 2$, yielding :

$$
\mathrm{J}_{\mathrm{ex}}=\theta
$$

This gives exchange constant $\mathrm{J}_{\mathrm{ex}} / \mathrm{k}_{\mathrm{B}}=-2.5 \mathrm{~K}$ using the mean-field theory and it is compared with $\mathrm{J}_{\mathrm{ex}} / \mathrm{k}_{\mathrm{B}}$ obtained from the Bonner-Fisher model discussed below. 


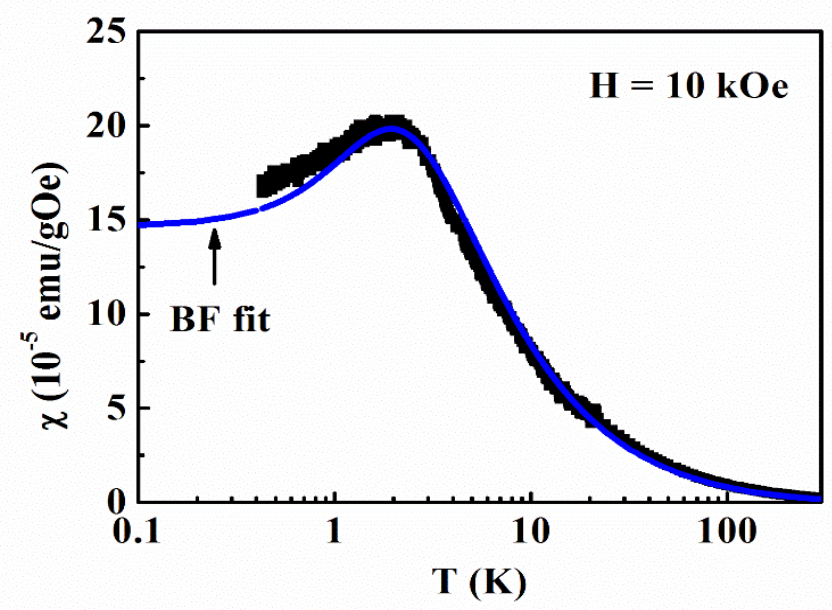

Fig. 5.7 Magnetic susceptibility $\chi$ vs. temperature T plot for $\beta$-CoPc. The solid line is fit to the BF model (Eq. 5.3) using $\chi_{o}=-1.8 \times 10^{-6} \mathrm{emu} / \mathrm{gOe}, \mathrm{C}=1.02 \times 10^{-3} \mathrm{emu}-\mathrm{K} / \mathrm{gOe}$, and $\mathrm{J}_{e x} / k_{B}=-1.5 \mathrm{~K}$.

\subsubsection{Fit to the Bonner-Fisher Model}

The data of $\chi$ vs. $\mathrm{T}$ for the whole temperature range of $0.4 \mathrm{~K}$ to $300 \mathrm{~K}$ was next fitted to the predictions of the Bonner-Fisher (BF) model [6] for a spin $S=1 / 2$ linear chain with Heisenberg AFM exchange-coupling $\mathrm{J}_{\mathrm{ex}}$ defined by the Hamiltonian $\mathrm{H}=-2 \mathrm{~J}_{\mathrm{ex}} \Sigma \mathbf{S}_{\mathbf{i}} \cdot \mathbf{S}_{\mathbf{i}+\mathbf{1}}$. Following Eggert et al. [7] and Feyerherm et al. [8], the variation of $\chi$ vs. $T$ for a linear chain model $S=1 / 2$ system with the normalized AFM coupling parameter $\mathrm{x}=\left|\mathrm{J}_{\mathrm{ex}}\right| /\left(\mathrm{k}_{\mathrm{B}} \mathrm{T}\right)$ is given by

$$
\begin{gathered}
\chi=\chi_{0}+\frac{C}{T} F(x) \\
F(x)=\frac{1+a_{1} x+a_{2} x^{2}}{1+b_{1} x+b_{2} x^{2}+b_{3} x^{3}}
\end{gathered}
$$

Here $C$ is the Curie constant, $x=\left|J_{\mathrm{ex}}\right| /\left(\mathrm{k}_{\mathrm{B}} \mathrm{T}\right)$ and $\mathrm{a}_{1}=0.17032, \mathrm{a}_{2}=0.93404, \mathrm{~b}_{1}=1.46764, \mathrm{~b}_{2}=0.54784$, and $b_{3}=4.28544$, similar to the analysis presented in Chapter 4 for the CuPc system which also has $\mathrm{S}=$ 1/2. With $\chi_{0}=-1.8 \times 10^{-6} \mathrm{emu} / \mathrm{gOe}$ and $\mathrm{C}=1.02 \times 10^{-3} \mathrm{emu}-\mathrm{K} / \mathrm{gOe}$ as determined above, the solid line in 
Fig. 5.7 is the best fit to Eq. (5.3) using $\mathrm{J}_{\mathrm{ex}} / \mathrm{k}_{\mathrm{B}}=-1.5 \mathrm{~K}$, within uncertainty of $0.1 \mathrm{~K}$ in the magnitudes of $\mathrm{J}_{\mathrm{ex}} / \mathrm{k}_{\mathrm{B}}$. As for the CuPc case in Chapter 4, the magnitude of $\mathrm{J}_{\mathrm{ex}}$ was varied till the best fit was obtained in the whole temperature range. In addition, using the observed $\mathrm{T}_{\max }=1.9 \mathrm{~K}=1.28\left|\mathrm{~J}_{\mathrm{ex}}\right| / \mathrm{k}_{\mathrm{B}}$ according to the BF model also yields $\left|\mathrm{J}_{\mathrm{ex}}\right| / \mathrm{k}_{\mathrm{B}}=1.5 \mathrm{~K}$. Thus the observation of $\mathrm{T}_{\max }$ and the overall fit of the data to Eq. (5.3) of the $\mathrm{BF}$ model with $\mathrm{J}_{\mathrm{ex}} / \mathrm{k}_{\mathrm{B}}=-1.5 \mathrm{~K}$ provides convincing evidence for the accuracy of this analysis. This magnitude of $\mathrm{J}_{\mathrm{ex}} / \mathrm{k}_{\mathrm{B}}$ is somewhat smaller than $\mathrm{J}_{\mathrm{ex}} / \mathrm{k}_{\mathrm{B}}=-1.9 \mathrm{~K}$ reported by Serri et al [2] which was determined from the fit of the $\mathrm{M} v \mathrm{vs} . \mathrm{H}$ data at $2 \mathrm{~K}$.

The CW law can be derived from Eq. (5.3) by retaining only the first order term in $\mathrm{x}$. This exercise leads to the CW law with $\theta=1.29732 \mathrm{~J}_{\mathrm{ex}} / \mathrm{k}_{\mathrm{B}}$. Using $\mathrm{J}_{\mathrm{ex}} / \mathrm{k}_{\mathrm{B}}=-1.5 \mathrm{~K}$ for $\beta$-CoPc yields $\theta=-2.0 \mathrm{~K}$ for $\beta$-CoPc, a little smaller than the experimental values of $\theta=-2.5 \mathrm{~K}$. This disagreement is due to the fact that at lower temperatures, higher order terms in $\mathrm{x}$ become increasingly important particularly for linear chains and 2-dimensional systems [9].

\subsubsection{Magnetic Field Dependence of Magnetization}

The data of $\mathrm{M}$ vs. $\mathrm{H}$ at $1 \mathrm{~K}$ and $5 \mathrm{~K}$ bracketing $\mathrm{T}_{\max }=1.9 \mathrm{~K}$ are shown in Fig. 5.8. The data at 1 $\mathrm{K}$ were acquired at the NHMFL, whereas the data at $5 \mathrm{~K}$ were taken at West Virginia University using the PPMS. The expected value of the saturation magnetization $\mathrm{M}_{\mathrm{S}}=\mathrm{Ng} \mu_{\mathrm{B}} \mathrm{S}$ is calculated to be $12.16 \mathrm{emu} / \mathrm{g}$ using $\mathrm{g}=2.49$ and $\mathrm{S}=1 / 2$ which agrees well with the measured value in Fig. 5.8 at $\mathrm{T}=1 \mathrm{~K}$ where the magnetization is nearly saturated. The Bonner-Fisher paper on the HLCA [5] does not provide an analytical expression for $\mathrm{M}$ vs. $\mathrm{H}$ but provides calculated plots of $\mathrm{M} v \mathrm{vs}$. $\mathrm{H}$ for several $\mathrm{k}_{\mathrm{B}} \mathrm{T} / \mathrm{J}_{\mathrm{ex}} \mid$ valid for $\mathrm{N}$ $=10$ spins in the chain. Using the internet-available software "digitizer", these plots were digitized. This 
software allows one to take a scanned image of a plot and digitizes the values off the plot by clicking the mouse on each data point. The data can then be saved and plotted. By following this procedure, we have digitized those plots from the original paper [6] and replotted them. Using $\left|\mathrm{J}_{\mathrm{ex}}\right| / \mathrm{k}_{\mathrm{B}} \mathrm{T}=1.5 \mathrm{~K}$ evaluated in this work from the fit of $\chi$ vs. T data, the red line (marked as $\mathrm{T}=0.9 \mathrm{~K}$ ) in Fig. 5.8 represents fit to the BF model for the available curve of $\mathrm{k}_{\mathrm{B}} \mathrm{T} /\left|\mathrm{J}_{\mathrm{ex}}\right|=0.6$ [5]. Overall, the agreement with the experimental data taken at $1 \mathrm{~K}$ is quite satisfactory considering that the $\mathrm{BF}$ curves are calculated for $\mathrm{N}=10$ spins in a chain rather the than for an infinite chain. The agreement is excellent for $\mathrm{H}<30 \mathrm{kOe}$, the region where the $\mathrm{BF}$ curves appear to be less sensitive to the magnitude of $\mathrm{J}_{\mathrm{ex}}$. This is the region used by Serri et al [2] to fit their data at $2 \mathrm{~K}$ to evaluate $\mid \mathrm{J}_{\mathrm{ex}} / \mathrm{k}_{\mathrm{B}}=1.9 \mathrm{~K}$. Because of this, it is suggested that the smaller magnitude of $\mid \mathrm{J}_{\mathrm{ex}} / \mathrm{k}_{\mathrm{B}}=1.5 \mathrm{~K}$ evaluated here from $\mathrm{T}_{\max }$ and the fit to the $\chi$ vs. $\mathrm{T}$ from $0.4 \mathrm{~K}$ to $300 \mathrm{~K}$ is likely more accurate.

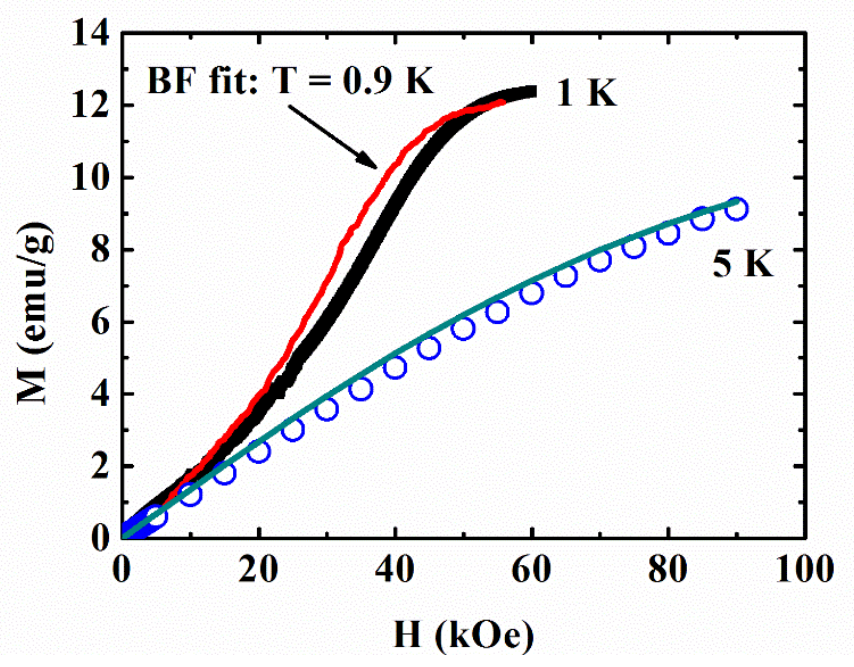

Fig. 5.8 Magnetization $M$ vs. $H$ data for $\beta$-CoPc at $1 K$ (black solid line) and $5 K$ (open circles) are compared respectively with the predictions of the Bonner-Fisher model at $0.9 \mathrm{~K}$ (red line) [from Ref. 6] and Eq. (5.5) for $5 \mathrm{~K}$ (blue line). 
For the $\mathrm{M}$ vs. $\mathrm{H}$ data at $5 \mathrm{~K}$, fitting the data to the $\mathrm{BF}$ model was not possible since the closest calculated available curve to $5 \mathrm{~K}$ from Ref. 6 is for $\mathrm{k}_{\mathrm{B}} \mathrm{T} / \mathrm{J}_{\mathrm{ex}} \mid=3.0$ which corresponds to $\mathrm{T}=4.5 \mathrm{~K}$. Since the fit of the $\chi$ vs. T data to the Curie-Weiss law is valid for $\mathrm{T}>3 \mathrm{~K}$ (Fig. 5.5), we have fitted the M vs. $\mathrm{H}$ data at $5 \mathrm{~K}$ to the prediction of the molecular-field model which for spin $\mathrm{S}=1 / 2$ yields the variation [10]

$$
M=M_{S} \tanh \frac{g \mu_{B} H}{2 k_{B}(T-\theta)}
$$

In the above, the $\mathrm{CW}$ variation is taken into account by including $\theta$. The fit of the data at $5 \mathrm{~K}$ to Eq. (5.5) using the experimental $\theta=-2.5 \mathrm{~K}, \mathrm{~g}=2.49$ and $\mathrm{M}_{\mathrm{S}}=12.16 \mathrm{emu} / \mathrm{g}$, shown as solid line in Fig. 5.8, is quite good.

\subsubsection{Temperature Dependence of AC Susceptibilities}

The temperature dependence of the ac susceptibilities were carried out for $\beta$-CoPc utilizing the in house PPMS. The ac susceptibilities for $\beta$-CoPc were measured from $2 \mathrm{~K}$ to $20 \mathrm{~K}$ with zero dc magnetic field but with a small ac magnetic field $\mathrm{H}_{\mathrm{ac}}=5$ Oe. The frequencies of the ac signals were 725 $\mathrm{Hz}, 1000 \mathrm{~Hz}, 3000 \mathrm{~Hz}, 4000 \mathrm{~Hz}$ and $5000 \mathrm{~Hz}$. As shown in Fig. 5.9, there is no frequency dependence for the real part of susceptibility $\chi^{\prime}$. For the imaginary part of susceptibility $\chi^{\prime \prime}$, there is a broad peak near $4 \mathrm{~K}$ for each measured frequency but without any measurable peak shift, which implies the relaxation process is associated with a much higher characteristic frequency than the measurement frequencies from $725 \mathrm{~Hz}$ to $5000 \mathrm{~Hz}$ used here for $\beta$-CoPc. 


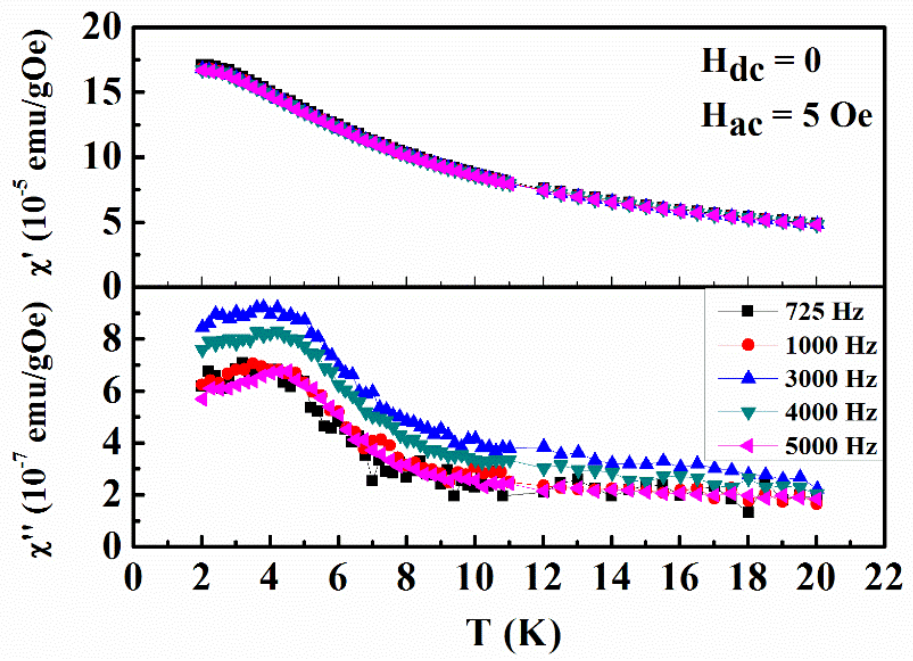

Fig. 5.9 Frequency dependence of the ac susceptibilities $\chi^{\prime}$ and $\chi^{\prime \prime}$ for $\beta$-CoPc measured in zero applied static field with $H_{a c}=5$ Oe at several frequencies between $725 \mathrm{~Hz}$ and $5000 \mathrm{~Hz}$.

\subsection{Conclusions}

The major conclusions from the results presented in this chapter are as follows: (i) The electronic state of $\mathrm{Co}^{2+}$ in $\beta$-CoPc is shown to have the effective spin $\mathrm{S}=1 / 2$ violating Hunds' rules. The resulting distribution of $3 \mathrm{~d}^{7}$ electrons of $\mathrm{Co}^{2+}$ is shown in Fig. 1.6. ; (ii) The fit of the magnetic susceptibility versus temperature data to the Curie-Weiss law yields $\theta=-2.5 \mathrm{~K}, \mu=2.16 \mu_{\mathrm{B}}$, and $\mathrm{g}=2.49$ for spin $S=1 / 2$ for $\beta$-CoPc. The $g$-shift results from the partially restored contribution of orbital moment via spin-orbit coupling; (iii) The fit of the magnetic susceptibility versus temperature data to the Bonner-Fisher model for linear Heisenberg chains including the observed peak at $\mathrm{T}_{\max }=1.9 \mathrm{~K}$ yields the Heisenberg exchange constant $\mathrm{J}_{\mathrm{ex}} / \mathrm{k}_{\mathrm{B}}=-1.5 \mathrm{~K}$ between the $\mathrm{Co}^{2+}-\mathrm{Co}^{2+}$ ions in $\beta$-CoPc; (iv) The isothermal data of $\mathrm{M}$ vs. $\mathrm{H}$ at $1 \mathrm{~K}$ shows good agreement with the Bonner-Fisher model. $\mathrm{M}$ vs. $\mathrm{H}$ at $5 \mathrm{~K}$ data is interpreted by including the effect of the exchange interaction; (v) The results show that $\beta$-CoPc is a good example of antiferromagnetically coupled Heisenberg linear chain with spin $\mathrm{S}=1 / 2$ of $\mathrm{Co}^{2+}$ and magnetic 
moment $\mu=2.16 \mu_{\mathrm{B}}$. This fit is obtained without invoking any interchain exchange coupling.

\section{References}

[1] W. Wu, N.M. Harrison and A.J. Fisher, Phys. Rev. B. 88, 024426 (2008).

[2] M. Serri, W. Wu, L.R. Fleet, N.W. Harrison, C.F. Hirjibehedin, C.W.M. Kay, A.J. Fisher, G. Aeppi and S. Heutz, Nat. Commun. 5, 3079 (2014).

[3] Z. Wang, M. Lee, E.S. Choi, J. Poston and M.S. Seehra, J. Magn. Magn. Mater. 407, 83 (2016).

[4] E.B. Faulkner and R.J. Schwartz, High Performance Pigments, $2^{\text {nd }}$ edition. Wiley-VCH, 2009.

[5] B.N. Figgs, E.S. Kucharski and P.A. Reynolds, J. Am. Chem. Soc. 111, 1683 (1989).

[6] J.C. Bonner and M.E. Fisher, Phys. Rev. 135, A640 (1964).

[7] S. Eggert, I. Affleck and M. Takahashi, Phys. Rev. Lett. 73, 332 (1994).

[8] R. Feyerhem, S. Abens, D. Gunther, T. Ishida, M. Meibner, M. Meschke, T. Nogami and M. Steiner, J. Phys. Condens. Matter. 12, 8495 (2000).

[9] M.S. Seehra, Phys. Lett. A. 28, 754 (1969).

[10] V. Narang, D. Korakakis and M.S. Seehra, J. Appl. Phys. 116, 213911 (2014). 


\section{CHAPTER 6.}

\section{Magnetic Properties of Vanadyl Phthalocyanine}

\subsection{Introduction}

Based on the Hunds' rules, the electron configuration of the unpaired electron of the $(\mathrm{VO})^{2+}$ ion in VOPc $\left(\mathrm{C}_{32} \mathrm{H}_{16} \mathrm{~N}_{8} \mathrm{VO}\right)$ is expected to be $3 \mathrm{~d}^{1}$ i.e. a single electron in the $3 \mathrm{~d}$ shell. According to a recent report [1], the nature of magnetism in VOPc $\left(\mathrm{C}_{32} \mathrm{H}_{16} \mathrm{~N}_{8} \mathrm{VO}\right)$ is simple paramagnetism. This report is in part based on the earlier work of Lever [2] which effectively list its magnetic moment $\mu=1.73 \mu_{\mathrm{B}}$, corresponding to $\mathrm{g}=2$ and $\mathrm{S}=1 / 2$ valid for a free electron. However, temperature and magnetic field dependence of the magnetization of VOPc has not been reported so far to verify such a designation. Therefore such measurements covering the temperature range of $0.5 \mathrm{~K}$ to $300 \mathrm{~K}$ in magnetic fields up to $90 \mathrm{kOe}$ were carried out in this work. Results from these measurements are presented and discussed below.

\subsection{Sample and its Structural Characterization}

The powder sample of VOPc was purchased from Sigma Aldrich (stock \# 791997) and used as received. X-ray diffraction (XRD) of the sample was performed using a PANalytical X'Pert Pro X-ray diffractometer with monochromatic $\mathrm{Cu}-\mathrm{K} \alpha$ radiation of wavelength $\lambda=0.15418 \mathrm{~nm}$. The XRD pattern of the sample is shown in Fig. 6.1 along with the Miller indices of the major Bragg lines. The positions of the Bragg lines were calculated using equations (3.6) through (3.13) of Chapter 3 valid for the triclinic structure and the lattice parameters listed below. It was found that the structure matched perfectly with 
that of phase II for VOPc having space group $\mathrm{P} \overline{1}$ with two molecules per unit cell of the triclinic structure and lattice parameters: $a=1.2027 \mathrm{~nm}, b=1.2571 \mathrm{~nm}, \mathrm{c}=0.8690 \mathrm{~nm}, \alpha=96.04^{\circ}, \beta=94.80^{\circ}$, and $\gamma=68.20^{\circ}$ [3]. This structure is shown in Fig. 6.2. In contrast to the other TMPc investigated here, VOPc is a nonplanar molecule and has a five- coordinate square pyramidal structure with a single electron associated with $\mathrm{VO}^{2+}$ ion [4]. By analyzing X-ray diffraction (XRD) data, Ziolo et al. showed that V atom is $0.0575 \mathrm{~nm}$ above the molecular plane and V-O distance is $0.1580 \mathrm{~nm}$ [3].

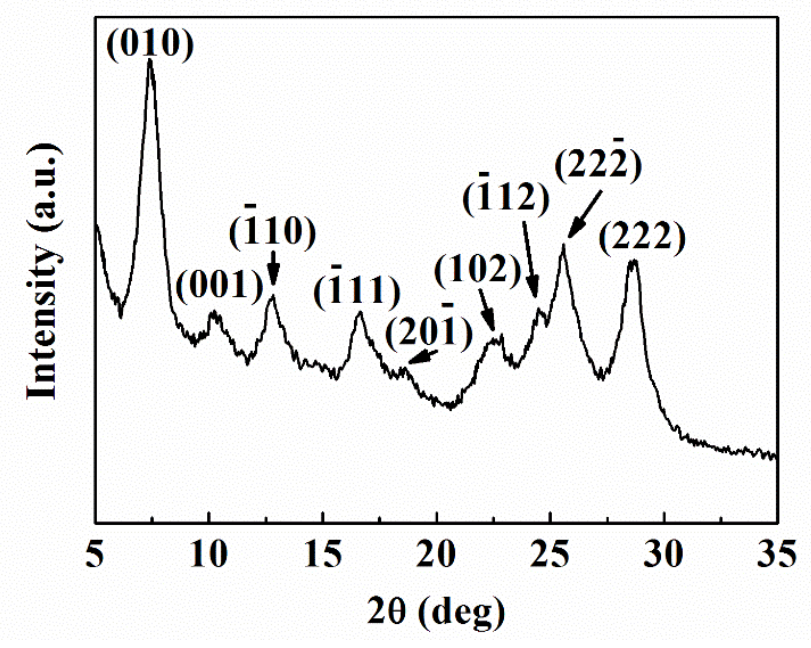

Fig. 6.1 XRD pattern of VOPc powder sample with Miller Indices of the peaks shown. 


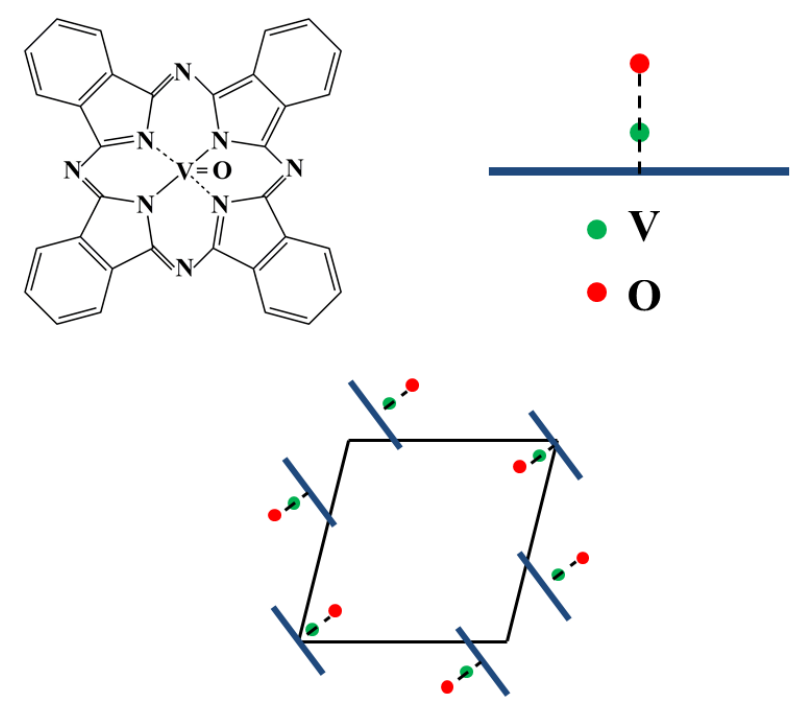

Fig. 6.2 Molecular structure and unit cell of VOPc (phase II). The lower panel shows a view down the $b$-axis of the unit cell. The blue lines in the unit cell depict the orientation of the molecular planes of VOPc.

The scanning electron microscopy of the VOPc powder sample was done using a JEOL 7600 FE-SEM system at the DOE National Energy Technology Laboratory. The SEM micrograph in Fig. 3 shows micron-sized agglomerated particles with some plate-like features of the particles. No additional information was obtainable from this micrograph. 


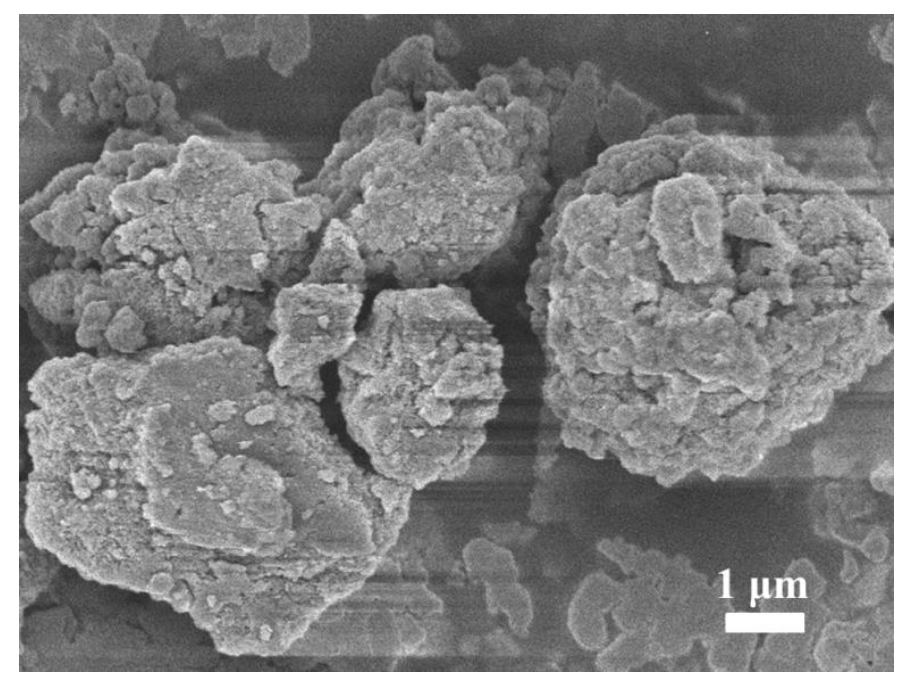

Fig. 6.3 A Scanning electron micrograph for the VOPc sample. The length of scale bar is $1 \mu m$.

\subsection{Results from Magnetic Measurements, Analysis, and Discussion}

\subsubsection{Temperature Dependence of DC Magnetization and the Curie-Weiss Law}

Measurements of the magnetization $\mathrm{M}$ of the VOPc sample as a function of temperature $\mathrm{T}$ from $2 \mathrm{~K}$ to $300 \mathrm{~K}$ and in magnetic field $\mathrm{H}$ up to $90 \mathrm{kOe}$ were performed at WVU using the in-house Physical Property Measurement System (PPMS) manufactured by Quantum Design Inc. In addition, performing magnetization measurements at temperatures lower than $2 \mathrm{~K}$ was considered quite desirable in this case since such data allows one to determine whether VOPc is a perfect paramagnet or there exists a small exchange coupling between $\mathrm{VO}^{2+}$ ions. The magnetization measurements down to $0.5 \mathrm{~K}$ in magnetic fields up to $70 \mathrm{kOe}$ were carried out in collaboration with Dr. Li Pi at the High Magnetic Field Laboratory, Chinese Academy of Sciences (CHMFL) in China using a Magnetic Property Measurement System (MPMS) acquired from Quantum Design Inc.

The magnetization measurements performed at the CHMFL are: $\mathrm{M}$ vs. $\mathrm{T}$ from $0.5 \mathrm{~K}$ to $2 \mathrm{~K}$ with 
$\mathrm{H}=1 \mathrm{kOe}$ and $\mathrm{M}$ vs. $\mathrm{H}$ up to $70 \mathrm{kOe}$ at $0.5 \mathrm{~K}, 1 \mathrm{~K}$, and $1.5 \mathrm{~K}$. The $\mathrm{M}$ vs. $\mathrm{T}$ data (from $0.5 \mathrm{~K}$ to $2 \mathrm{~K}$ ) acquired at the CHMFL was normalized to the $\mathrm{M}$ vs. $\mathrm{T}$ data (from $0.5 \mathrm{~K}$ to $300 \mathrm{~K}$ ) acquired using the in-house PPMS at a single temperature $(2 \mathrm{~K})$. Fig 6.4 shows the combined data of the temperature dependence of magnetic susceptibility $\chi=\mathrm{M} / \mathrm{H}$ for $\mathrm{VOPc}$ powder sample measured at $\mathrm{H}=1 \mathrm{kOe}$ covering the temperature range of $0.5 \mathrm{~K}$ to $300 \mathrm{~K}$.

The analysis of the data shown in Fig. 6.4 was done as follows. First the $\chi$ vs. T data from $0.5 \mathrm{~K}$ to $300 \mathrm{~K}$ was fitted to the modified Curie-Weiss $(\mathrm{CW})$ law: $\chi=\chi_{0}+\mathrm{C} /(\mathrm{T}-\theta)$. For this purpose, $\chi_{0}=-9.3$ $\times 10^{-7} \mathrm{emu} / \mathrm{gOe}$ was determined by plotting $\chi$ vs. $1 / \mathrm{T}$ and linearly extrapolating the high temperature data to $1 / \mathrm{T} \rightarrow 0$, as shown in Fig. 6.5. As in the cases of $\mathrm{CuPc}$ and $\mathrm{CoPc}, \chi_{0}$ contains diamagnetic contributions of both VOPc as well as the plastic sample holder used in the measurements. However $\chi_{\mathrm{o}}$ is quite small compared to the measured $\chi$ for $\mathrm{T}<100 \mathrm{~K}$ and so its effects become only noticeable at temperatures $\mathrm{T}>$ $100 \mathrm{~K}$.

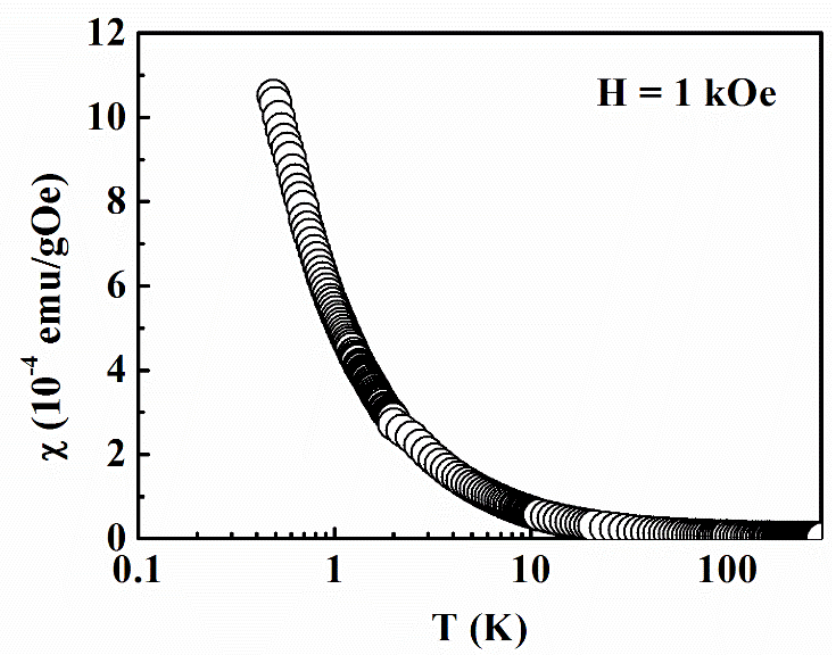

Fig. 6.4 Magnetic susceptibility $\chi$ vs. temperature T of VOPc powder sample. 


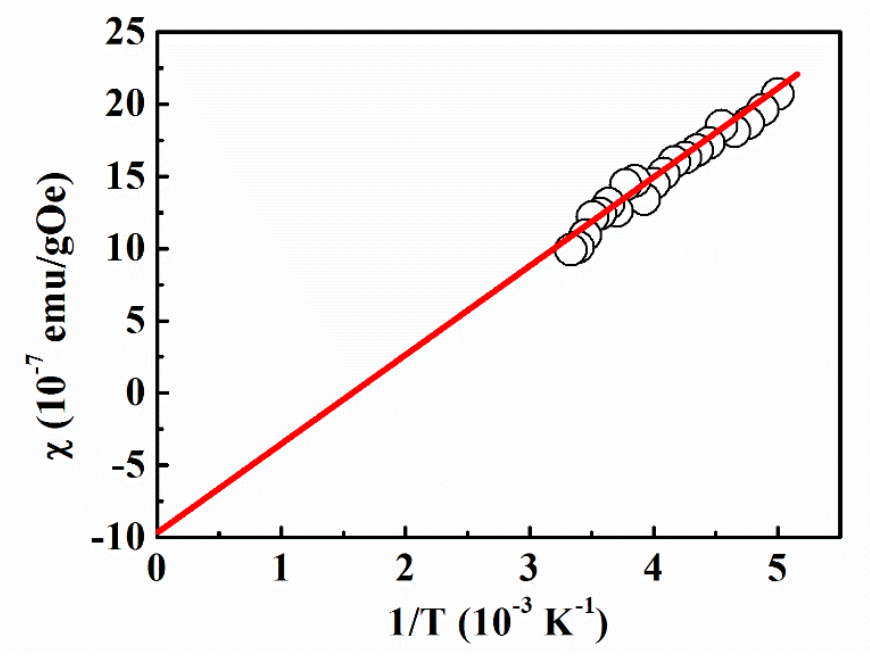

Fig. 6.5 Plot of magnetic susceptibility $\chi$ vs. $1 /$ T for VOPc with $H=1 \mathrm{kOe}$. The solid line is the linear fit of the experimental data and its intercept with y axis $(\chi)$ gives $\chi_{o}=-9.3 \times 10^{-7}$ emu/gOe.

After determining $\chi_{0}$, the data of $\left(\chi-\chi_{0}\right)^{-1}$ was plotted against temperature (Fig. 6.6) yielding a straight line whose slope $=1 / \mathrm{C}$ yielded $\mathrm{C}=6.266 \times 10^{-4} \mathrm{emu}-\mathrm{K} / \mathrm{gOe}$. The intercept on the T-axis yields $\theta$ $=-0.1 \mathrm{~K}$ within the experimental uncertainty, the negative $\theta$ signifying antiferromagnetic exchange coupling.

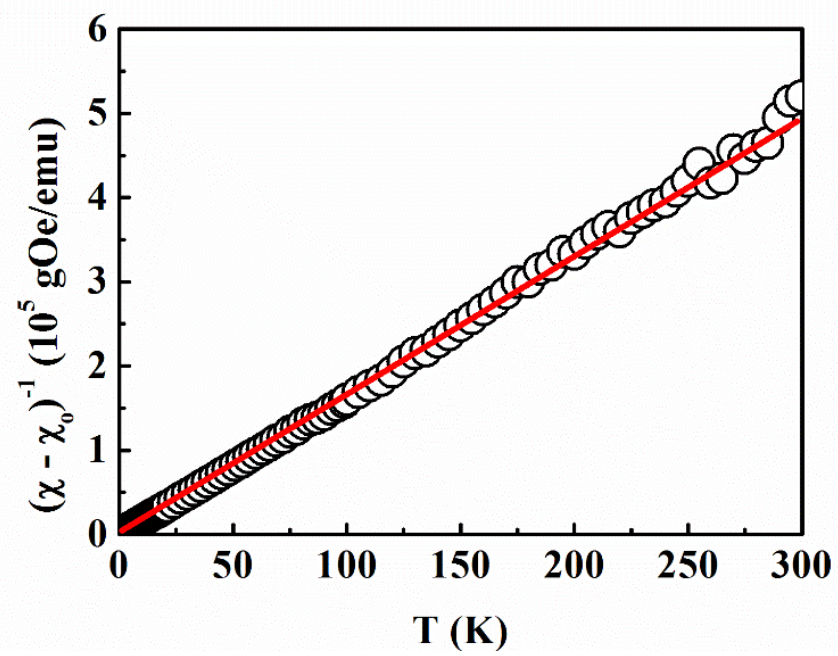

Fig. 6.6 Plot of temperature dependence of the inverse magnetic susceptibility $\left(\chi-\chi_{o}\right)^{-1}$ of VOPc in $H=1$ $k O e$. The solid line is the linear fit representing $C W$ law. 
The solid lines in Fig. 6.7 are fits to the modified CW law shown for both $\theta=-0.1 \mathrm{~K}$ and $\theta=0$ $\mathrm{K}$ with $\chi_{\mathrm{o}}=-9.3 \times 10^{-7} \mathrm{emu} / \mathrm{gOe}$ and $\mathrm{C}=6.266 \times 10^{-4} \mathrm{emu}-\mathrm{K} / \mathrm{gOe}$. It is evident that the choice of $\theta=-$ $0.1 \mathrm{~K}$ provides a better fit to the data especially for $\mathrm{T}<2 \mathrm{~K}$. This magnitude of $\theta=-0.1 \mathrm{~K}$ represents a very weak antiferromagnetic exchange coupling between the $\mathrm{VO}^{2+}$ ions and the so near-perfect paramagnetic nature of VOPc. In comparison, in the corresponding systems of $\alpha$-CuPc and $\beta$-CuPc [5] and $\beta$-CoPc [6], all with $S=1 / 2$, considerably larger magnitudes of $\theta=-2.3 \mathrm{~K},-0.2 \mathrm{~K}$, and $-2.5 \mathrm{~K}$ respectively were observed. Assuming a linear chain antiferromagnetic system as in $\alpha$-CuPc and $\beta$-CuPc [5] and $\beta$-CoPc [6], yields $\theta=1.29732 \mathrm{~J}_{\mathrm{ex}} / \mathrm{k}_{\mathrm{B}}$ where $\mathrm{J}_{\mathrm{ex}}$ is the Heisenberg exchange constant in the Hamiltonian $\mathrm{H}=-2 \mathrm{~J}_{\mathrm{ex}} \sum \mathbf{S}_{\mathrm{i}} \cdot \mathbf{S}_{\mathrm{i}+1}$ and $\mathrm{k}_{\mathrm{B}}$ is the Boltzmann constant. This analysis yields $\mathrm{J}_{\mathrm{ex}} / \mathrm{k}_{\mathrm{B}}=-0.077$ $\mathrm{K}$ as the exchange coupling constant in VOPc for $\theta=-0.1 \mathrm{~K}$. It is evident in Fig. 6.7 that without the $\chi$ vs. T measurements reported here for $\mathrm{T}<2 \mathrm{~K}$, a case for $\theta=-0.1 \mathrm{~K}$ could not have been made.

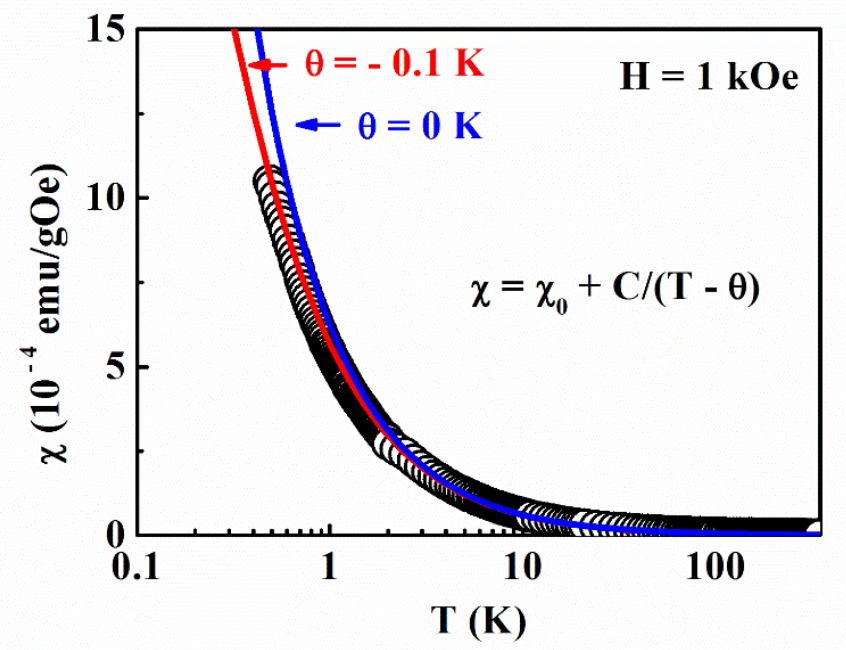

Fig. 6.7 Magnetic susceptibility $\chi$ versus temperature $T$ from $0.5 \mathrm{~K}$ to $300 \mathrm{~K}$ with $\mathrm{H}=1 \mathrm{kOe}$ of VOPc powder sample. The open circles are experimental data and the solid lines are fits to the modified Curie-Weiss law with the parameters: $\chi_{o}=-9.3 \times 10^{-7} \mathrm{emu} / \mathrm{gOe}, \mathrm{C}=6.266 \times 10^{-4} \mathrm{emu}-\mathrm{K} / \mathrm{gOe}$, and $\theta=-$ $0.1 \mathrm{~K}$ and $0 \mathrm{~K}$ as shown. 
Using $C=N \mu^{2} / 3 k_{B}$ where $N$ is the number of magnetic ion per gm and $\mu$ is the magnetic moment per ion, the evaluated $\mathrm{C}=6.266 \times 10^{-4} \mathrm{emu}-\mathrm{K} / \mathrm{gOe}$ yields magnetic moment $\mu=1.704 \mu_{\mathrm{B}}$ per $\mathrm{VO}^{2+}$ ion. The molecular weight $(\mathrm{MW})=579.46 \mathrm{~g} / \mathrm{mol}$ for $\mathrm{VOPc}$ is used in these calculations to determine $N=N_{A} / M W$ where $N_{A}$ is the Avogadro's number. This magnitude of $\mu=1.704 \mu_{B}$ shows the electronic state of V in VOPc is indeed $\mathrm{V}^{4+}$ (electronic configuration: $3 \mathrm{~d}^{1}$ ) corresponding to spin $\mathrm{S}=1 / 2$

(see table 1.2). For $S=1 / 2, g=1.967$ is obtained using $\mu^{2}=g^{2} S(S+1) \mu_{B}^{2}$. The magnitude of $g<2$ due to orbital contribution is expected for TM ions with d-shell less than half full as is valid for $\mathrm{V}^{4+}$ or $\mathrm{VO}^{2+}$.

Electron spin resonance (ESR) is a complementary technique to magnetic measurement to accurately determine the g-values. Very early brief report by Ingram and Bennet [8] in VOPc reported a broad absorption with $\mathrm{g}=2$ with a width of about 500 Oe. Assour et al [9] reported ESR studies in VOPc diluted $(1: 1000)$ in $\mathrm{H}_{2} \mathrm{SO}_{4}, \mathrm{H}_{2} \mathrm{Pc}$ and $\mathrm{ZnPc}$ yielding $\mathrm{g}_{\|}=1.965$ and $\mathrm{g}_{\perp}=1.988$ yielding an average $\mathrm{g}=\left(\mathrm{g}_{\|}\right.$ $\left.+2 g_{\perp}\right) / 3=1.980$. This g-value matches well with $g=1.967$ determined here from the magnetic data. To the best of our knowledge, studies on the temperature dependence of the ESR spectra of undiluted VOPc have not yet been reported which would provide a better comparison with our magnetic data. At our suggestion, such ESR measurements are now being undertaken by the research group of Professor Dalal at Florida State University.

\subsubsection{Magnetic Field Dependence of Magnetization}

The isothermal data of $\mathrm{M}$ vs. $\mathrm{H}$ up to $90 \mathrm{kOe}$ measured at $0.5 \mathrm{~K}, 1 \mathrm{~K}, 1.5 \mathrm{~K}, 2 \mathrm{~K}, 5 \mathrm{~K}, 10 \mathrm{~K}, 25$

$\mathrm{K}, 50 \mathrm{~K}, 100 \mathrm{~K}$, and $300 \mathrm{~K}$ for VOPc are shown in Fig. 6.8. To verify the near-perfect paramagnetism of VOPc, the isothermal data of $M$ vs. $H$ for all temperatures is plotted in terms of $M$ vs. $H /(T-\theta)$ in Fig. 
6.9 to check its Brillouin function variation expected for $S=1 / 2$ systems given by [7]:

$$
M=M_{S} \tanh \frac{g \mu_{B} H}{2 k_{B}(T-\theta)}
$$

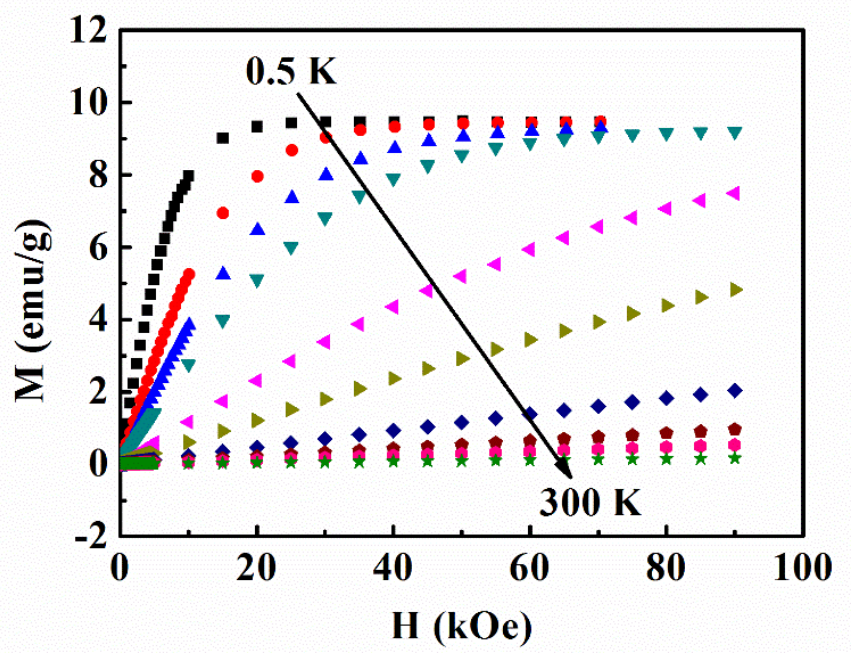

Fig. 6.8 Magnetization $M$ vs. magnetic field $\mathrm{H}$ data for VOPc powder at $0.5 \mathrm{~K}, 1 \mathrm{~K}, 1.5 \mathrm{~K}, 2 \mathrm{~K}, 5 \mathrm{~K}, 10 \mathrm{~K}$, $25 \mathrm{~K}, 50 \mathrm{~K}, 100 \mathrm{~K}$, and $300 \mathrm{~K}$. Note that the data at $0.5 \mathrm{~K}, 1 \mathrm{~K}$, and $1.5 \mathrm{~K}$ are with $\mathrm{H}$ up to $70 \mathrm{kOe}$; data at other temperatures are with $\mathrm{H}$ up to $90 \mathrm{kOe}$.

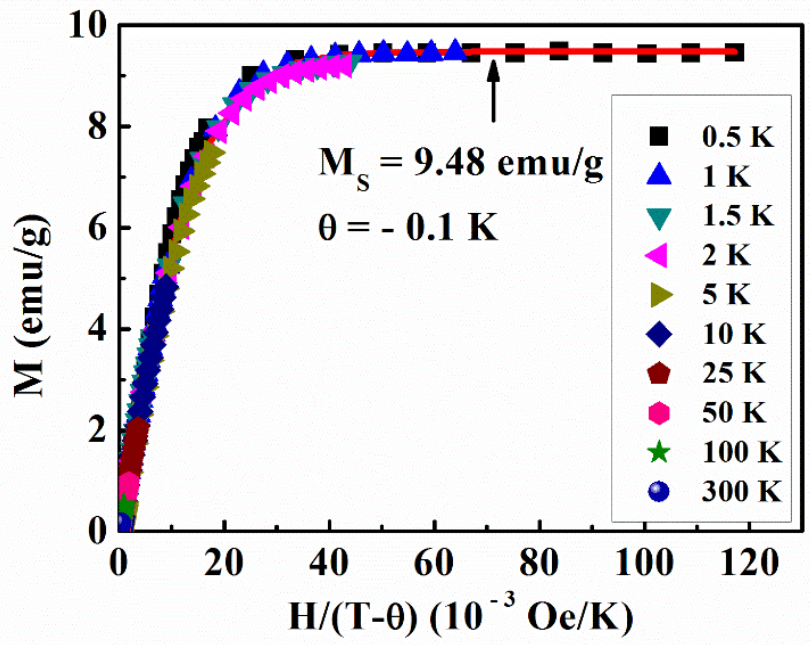

Fig. 6.9 Plot of magnetization $M v s . H /(T-\theta)$ with $\theta=-0.1 \mathrm{~K}$ for VOPc at $0.5 \mathrm{~K}, 1 \mathrm{~K}, 1.5 \mathrm{~K}, 2 \mathrm{~K}, 5 \mathrm{~K}, 10$ $\mathrm{K}, 25 \mathrm{~K}, 50 \mathrm{~K}, 100 \mathrm{~K}$, and $300 \mathrm{~K}$. The solid line (red color) is fit to Eq. (6.1) with $\mathrm{g}=1.967, M_{S}=9.48$ $e m u / g$, and $\theta=-0.1 \mathrm{~K}$. 
This plot in Fig. 6.9 shows collapsing of all the data on to a single curve which fits Eq. (6.1) very well with $\theta=-0.1 \mathrm{~K}$ implying the very weak antiferromagnetic exchange coupling between the $\mathrm{VO}^{2+}$ ions. Notice that saturation magnetization $\mathrm{M}_{\mathrm{S}}=9.46 \mathrm{emu} / \mathrm{g}$ at $0.5 \mathrm{~K}$ agrees well with the calculated value of $\mathrm{M}_{\mathrm{S}}=9.48 \mathrm{emu} / \mathrm{g}$ using $\mathrm{M}_{\mathrm{S}}=\mathrm{Ng} \mu_{\mathrm{B}} \mathrm{S}$ with $\mathrm{g}=1.967$ and $\mathrm{S}=1 / 2$. As noted earlier, the molecular weight $(\mathrm{MW})=579.46 \mathrm{~g} / \mathrm{mol}$ for VOPc is used in these calculations to determine $\mathrm{N}=\mathrm{N}_{\mathrm{A}} / \mathrm{MW}$ where $\mathrm{N}_{\mathrm{A}}$ is the Avogadro's number.

\subsection{Conclusions}

The major conclusions are summarized as following: (i) The electronic state of V in VOPc is shown to be $\mathrm{V}^{4+}$ with spin $\mathrm{S}=1 / 2$; (ii) The fit of the magnetic susceptibility versus temperature data to the Curie-Weiss law yields $\theta=-0.1 \mathrm{~K}, \mu=1.704 \mu_{\mathrm{B}}$, and $\mathrm{g}=1.967$ for $\operatorname{spin} \mathrm{S}=1 / 2$ for VOPc. $\theta=-0.1 \mathrm{~K}$ indicates a very weak antiferromagnetic exchange coupling between $\mathrm{VO}^{2+}$ ions which implies the near-perfect paramagnetism of VOPc. (iii) The negative g-shift from the free electron value is expected for shells less than half full as is valid for the $3 \mathrm{~d}^{1}$ configuration of $\mathrm{VO}^{2+}$ ions and it results from the partial contribution of the orbital moment via spin-orbit coupling. This is different from the expected positive g-shift reported here for $\mathrm{Cu}^{2+}$ and $\mathrm{Co}^{2+}$ ions in $\mathrm{CuPc}$ and $\mathrm{CoPc}$ systems with the electronic configurations of $3 d^{9}$ and $3 d^{7}$ respectively with $3 d$-shells more than half full; (iv) The collapsing of all the data of M vs. $\mathrm{H}$ to a single curve which fits Eq. (6.1) very well with $\theta=-0.1 \mathrm{~K}$ in terms of the plot of $\mathrm{M}$ vs. $\mathrm{H} /(\mathrm{T}-\theta)$ also confirms near perfect paramagnetism in VOPc with very weak exchange coupling between $\mathrm{VO}^{2+}$ ions. The experimental magnitude of saturation magnetization $\mathrm{M}_{\mathrm{S}}=9.46 \mathrm{emu} / \mathrm{g}$ at $0.5 \mathrm{~K}$ agrees very well with the calculated magnitude assuming complete alignment of the spins; (v) Measurements of the magnetic 
susceptibility versus temperature down to $0.5 \mathrm{~K}$ and $\mathrm{M}$ vs. $\mathrm{H}$ measurements at $0.5 \mathrm{~K}$ in VOPc were crucial in determining the accurate magnitudes of $\mu=1.704 \mu_{\mathrm{B}}, \mathrm{g}=1.967$ and $\theta=-0.1 \mathrm{~K}$ in this system.

\section{References}

[1] K. Eguchi, T. Takagi, T. Nakagawa and T. Yokoyama, J. Chem. Phys. C. 118, 17633 (2014).

[2] A.B.P. Lever, J. Chem. Soc. 1861 (1965).

[3] R.F. Ziolo, C.H. Griffiths and J.M. Troup, J. Chem. Soc., Dalton Trans. 11, 2300 (1980).

[4] H. Adler, M. Paszkiewicz, J. Uihlein, M. Polek, R. Ovsyannikov, T.V. Basova, T. Chasse and H. Peisert, J. Phys. Chem. C. 119, 8755 (2015).

[5] Z. Wang, K.L. Pisane and M.S. Seehra, IEEE. Trans. Mag. 51, 2700104 (2015).

[6] Z. Wang, M. Lee, E.S. Choi, J. Poston and M.S. Seehra, J. Magn. Magn. Mater. 407, 83 (2016).

[7] V. Narang, D. Korakakis and M.S. Seehra, J. Appl. Phys. 116, 213911 (2014).

[8] D.G.E. Ingram and J. E. Bennett, J. Chem. Phys. 22, 1136 (1954).

[9] J. M. Assour, J. Goldmacher and S.E. Harrison, J. Chem. Phys. 43, 159 (1965). 


\section{CHAPTER 7.}

\section{Magnetic Properties of $\beta$ - Nickel Phthalocyanine}

\subsection{Introduction}

The nature of magnetism in $\beta-\mathrm{NiPc}$ has been reported to be diamagnetism with the unusual low spin state $\mathrm{S}=0$ for $\mathrm{Ni}^{2+}\left(3 \mathrm{~d}^{8}\right)$ [1-4] as shown in Fig. 1.5 (chapter 1). However, detailed temperature and magnetic field dependence of the magnetization were not provided in these reports. In this chapter, results from the measurement of the magnetization $(\mathrm{M})$ of a $\beta$-NiPc sample from $2 \mathrm{~K}$ to $300 \mathrm{~K}$ and in magnetic fields $\mathrm{H}$ up to $90 \mathrm{kOe}$ are reported, analyzed and discussed.

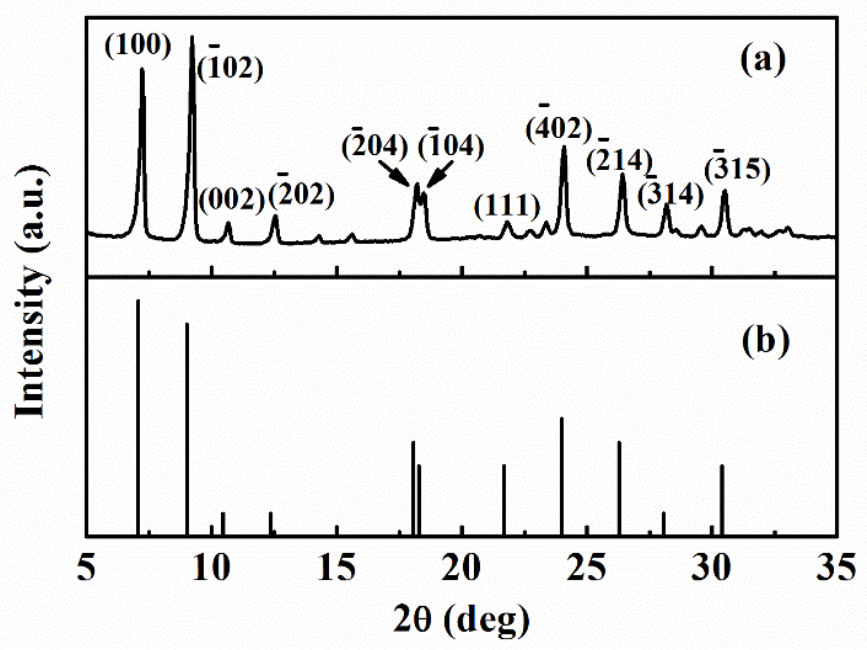

Fig. 7.1 The top panel shows the measured XRD pattern of $\beta$-NiPc sample with Miller indices of major lines marked; the bottom panel shows the expected line positions and intensities according to the ICDD data base of $\beta$-NiPc.

\subsection{Sample and its Structural Characterization}

The powder sample of NiPc used here was purchased from Alfa Aesar (stock \# 39453) and used 
as received. Fig. 7.1 shows the X-ray diffraction (XRD) of the sample measured at room temperature. This pattern agrees with the expected pattern of $\beta$-NiPc (ICDD \#00-011-0744) with space group P2 ${ }_{1} / \mathrm{c}$ and two molecules per unit cell in the monoclinic structure with lattice parameters: $a=1.49 \mathrm{~nm}, \mathrm{~b}=0.47 \mathrm{~nm}$, $\mathrm{c}=1.99 \mathrm{~nm}$, and $\beta=121.15^{\circ}$. This structure was shown in Fig. 1.1 of Chapter 1 . Note that all the observed peaks in the XRD pattern match well with the ICDD pattern for $\beta$-NiPc.

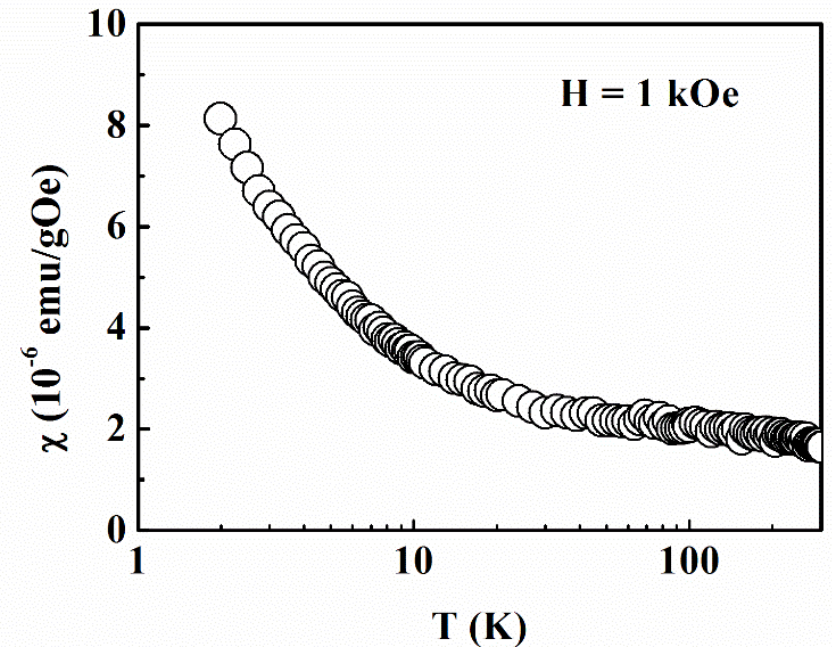

Fig. 7.2 Magnetic susceptibility $\chi$ of $\beta$-NiPc vs. temperature Twith $H=1 \mathrm{kOe}$.

\subsection{Results from Magnetic Measurements, Analysis, and Discussion}

\subsubsection{Temperature Dependence of DC Magnetization}

The temperature dependence of the magnetic susceptibility $\chi=\mathrm{M} / \mathrm{H}$ of the sample measured with $\mathrm{H}=1 \mathrm{kOe}$ is shown in Fig. 7.2. This temperature dependence is similar to that of a paramagnet although the measured magnetic susceptibility is about two orders of magnitude smaller than that observed in other TMPc samples. For example, $\chi$ at $2 \mathrm{~K}$ is of the order of $10^{-6} \mathrm{emu} / \mathrm{gOe}$ for $\beta$-NiPc compared to $10^{-4} \mathrm{emu} / \mathrm{gOe}$ for the CuPc samples at $2 \mathrm{~K}$. Since $\beta$-NiPc should be diamagnetic because of 
the spin $\mathrm{S}=0$ state, the $\chi$ vs. $\mathrm{T}$ data indicates the possible presence of a paramagnetic or ferromagnetic impurity (e.g. Fe) in the sample. Since the presence of an impurity was not observed in the XRD pattern, the magnitude of this impurity has to be smaller than about $3 \%$, the lower detection limit of XRD. Therefore additional magnetic measurements were undertaken to try to determine the nature of this impurity. These additional measurements and their discussion and analysis are presented below.

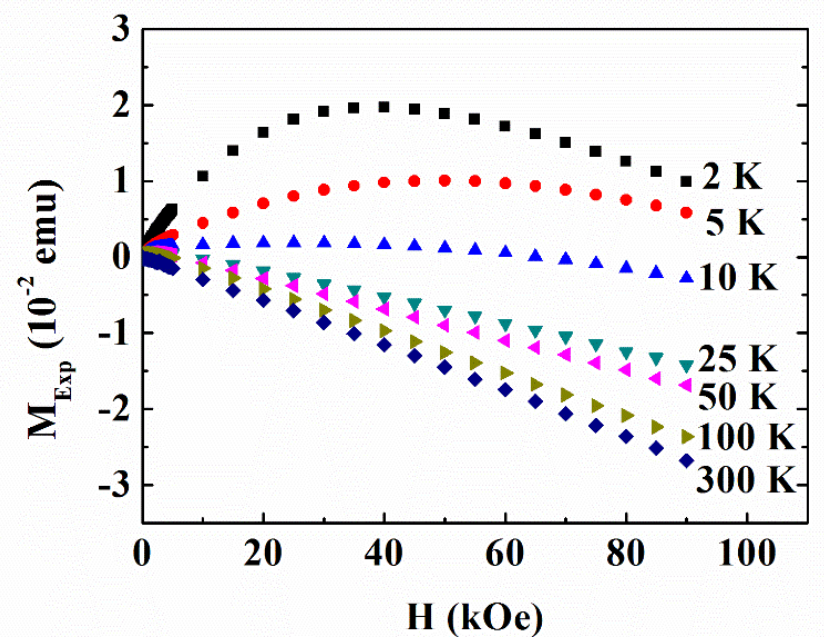

Fig. 7.3 Magnetization $M$ vs. field $H$ at temperatures indicated for $\beta$-NiPc plus the sample holder.

\subsubsection{Magnetic Field Dependence of Magnetization}

The isothermal data of $\mathrm{M}$ vs. $\mathrm{H}$ up to $90 \mathrm{kOe}$ measured at $2 \mathrm{~K}, 5 \mathrm{~K}, 10 \mathrm{~K}, 25 \mathrm{~K}, 50 \mathrm{~K}, 100 \mathrm{~K}$, and $300 \mathrm{~K}$ for the $\beta$-NiPc powder sample are shown in Fig. 7.3. At the higher temperatures of $25 \mathrm{~K}$ to 300 $\mathrm{K}$, the $\mathrm{M}$ vs. $\mathrm{H}$ variation is characteristic of a diamagnetic material with diamagnetic susceptibility decreasing with decrease in temperature. However from the data at the lower temperatures, it is evident that some ferromagnetic impurity must exist in the sample because of the visible rise of magnetization with increasing $\mathrm{H}$ at $2 \mathrm{~K}, 5 \mathrm{~K}$, and $10 \mathrm{~K}$. Also, it is noted that the measured magnetization is a 
combination from both $\beta$-NiPc and the sample holder. The sample holder is made of plastic and should be diamagnetic.

In order to obtain the data just from $\beta$-NiPc without any contribution from the sample holder, $\mathrm{M}$ vs. $\mathrm{H}$ measurements at $2 \mathrm{~K}, 5 \mathrm{~K}, 10 \mathrm{~K}, 25 \mathrm{~K}, 50 \mathrm{~K}, 100 \mathrm{~K}$, and $300 \mathrm{~K}$ for just the sample holder were performed and the data are shown in Fig. 7.4. The fact that the data at each temperature almost follows the same slope (magnetic susceptibility $\chi$ ) is understandable because the magnetic susceptibility $\chi$ should be independent of magnetic field and temperature for diamagnetic materials. Next, the magnetization $\mathrm{M}$ data for just $\beta$-NiPc at various temperatures were obtained (shown in Fig. 7.5) by subtracting the measured magnetization of the sample holder shown in Fig. 7.4 from the measured magnetization of NiPc plus sample holder shown in Fig. 7.3. The resulting magnetic field dependence of the magnetization of NiPc at different temperatures, now normalized by the mass of NiPc, is shown in Fig. 7.5.

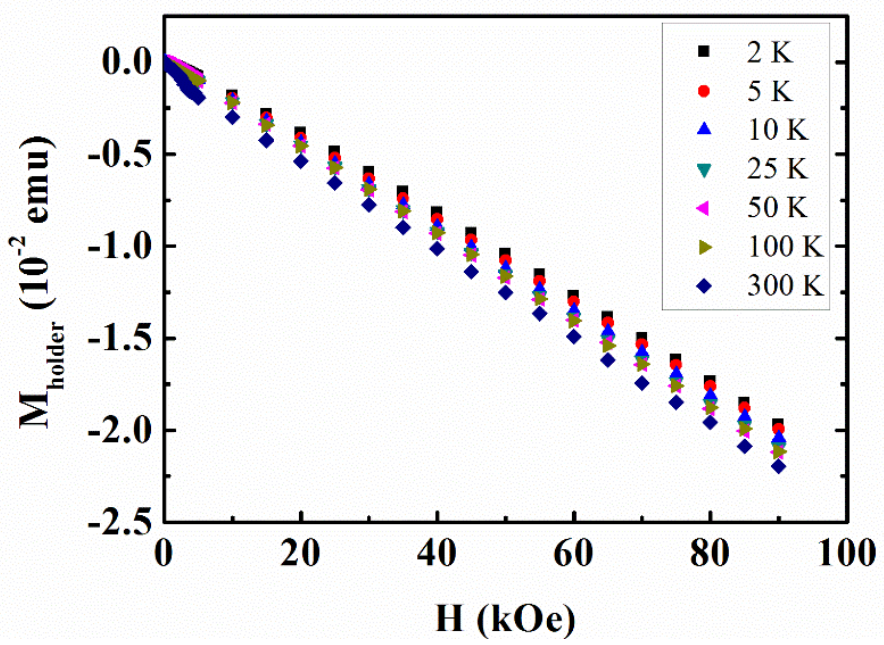

Fig. 7.4 Magnetization $M v$ s. field $H$ at several temperatures for just the sample holder. 


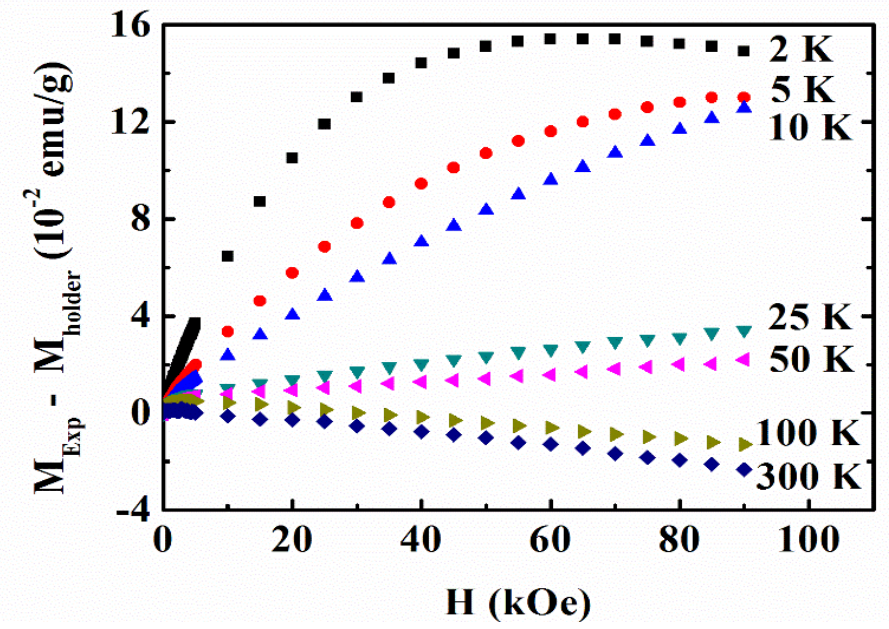

Fig. 7.5 The magnetization $M$ vs. field H data just for $\beta$-NiPc at temperatures shown on the plot.

The magnetization data of $\beta$-NiPc shown in Fig. 7.5 clearly has a ferromagnetic component $\left(\mathrm{M}_{\mathrm{FM}}\right)$ superposed on the diamagnetic component $\left(\mathrm{M}_{\mathrm{D}}\right)$ with the $\mathrm{M}_{\mathrm{FM}}$ essentially absent from the data at $300 \mathrm{~K}$. Therefore, the data were analyzed by using the following Eq.:

$$
\mathrm{M}_{\text {sample }}=\mathrm{M}_{\mathrm{D}}+\mathrm{M}_{\mathrm{FM}}=\chi_{\mathrm{D}} \mathrm{H}+\mathrm{M}_{\mathrm{FM}}
$$

Here $\chi_{\mathrm{D}}$ is the diamagnetic susceptibility of pure $\beta-\mathrm{NiPc}$ and should be the same at all temperatures and $\mathrm{M}_{\mathrm{FM}}$ is magnetization of the impurity. The linear fit of the data for $\mathrm{M}$ vs. $\mathrm{H}$ at $300 \mathrm{~K}$ yields the slope $\chi_{\mathrm{D}}=-$ $3.38 \times 10^{-7} \mathrm{emu} / \mathrm{gOe}$ (shown in Fig. 7.6). Then the ferromagnetic component $\mathrm{M}_{\mathrm{FM}}$ for each measured temperature can be determined by:

$$
\mathrm{M}_{\mathrm{FM}}=\mathrm{M}_{\text {sample }}-\chi_{\mathrm{D}} \mathrm{H}
$$

The magnetic field dependence of $\mathrm{M}_{\mathrm{FM}}$ was then determined at different temperatures and this temperature dependence of $\mathrm{M}_{\mathrm{FM}}$ and $\mathrm{M}_{\mathrm{D}}$ is shown in Fig.7.7. Assuming $\mathrm{M}_{\mathrm{D}}$ to be temperature 
independent as expected from theory, $\mathrm{M}_{\mathrm{FM}}$ increases with decrease in temperature.

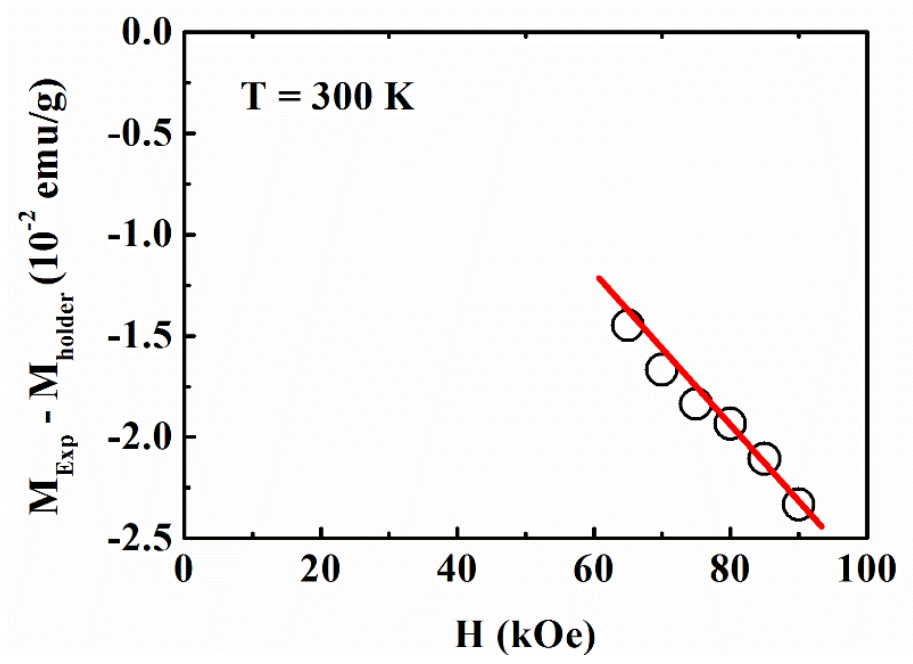

Fig. 7.6 Plot of magnetization $M$ vs. H for $\beta$-NiPc sample at $T=300 \mathrm{~K}$. The solid line is the linear fit of the experimental data for higher $H$ and the slope gives $\chi_{D}=-3.38 \times 10^{-7} \mathrm{emu} / \mathrm{gOe}$.

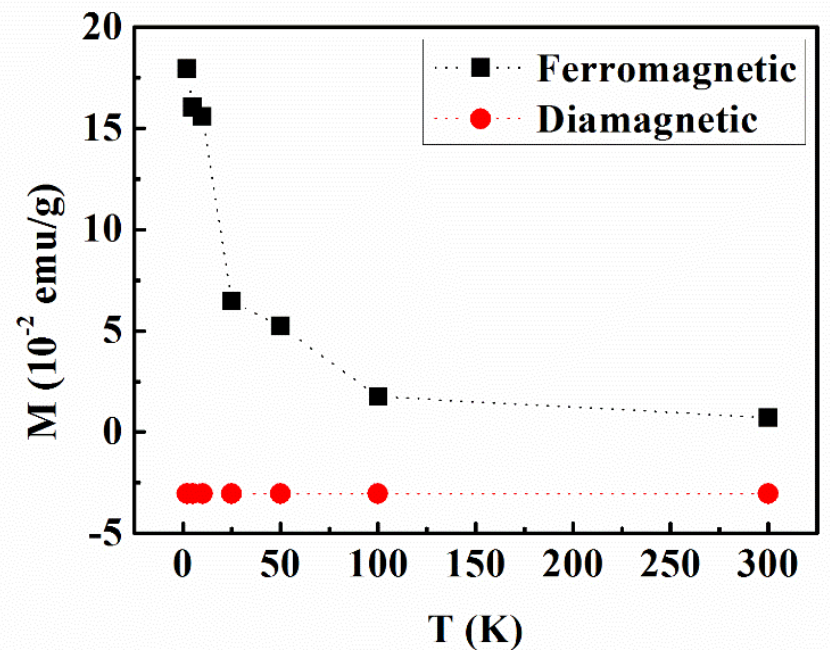

Fig. 7.7 Temperature dependence of the ferromagnetic component $M_{F M}$ and diamagnetic component $M_{D}$ for $\beta$-NiPc. The dotted lines connecting the data points are visual guides.

To verify the ferromagnetic character of the impurity, hysteresis loop measurements between + $90 \mathrm{kOe}$ and $-90 \mathrm{kOe}$ were carried out at $2 \mathrm{~K}, 5 \mathrm{~K}, 10 \mathrm{~K}, 25 \mathrm{~K}, 50 \mathrm{~K}, 100 \mathrm{~K}$, and $300 \mathrm{~K}$ for the $\beta$-NiPc 
sample. Fig. 7.8 shows the hysteresis loop at $2 \mathrm{~K}$ yielding the coercivity $\mathrm{H}_{\mathrm{C}}=250$ Oe. Similar hysteresis loops and coercivity were observed at $5 \mathrm{~K}, 10 \mathrm{~K}, 25,50 \mathrm{~K}$, and $100 \mathrm{~K}$. The hysteresis data at $300 \mathrm{~K}$ was very noisy and not shown here. Fig. 7.9 shows the plot of coercivity $\mathrm{H}_{\mathrm{C}}$ vs. temperature $\mathrm{T}$ at various temperatures from $2 \mathrm{~K}$ to $100 \mathrm{~K}$.

Although the ferromagnetic nature of the magnetic impurity is quite evident from these measurements, it is not possible to identify the chemical phase of the impurity. Therefore attempts were made to detect the magnetic impurity using x-ray photoelectron spectroscopy (XPS) of the sample. XPS was performed using a PHI 5000 VersaProbe XPS with Al-K $\alpha$ source $(\mathrm{h} v=1486.7 \mathrm{eV})$. The survey scan was performed for $\beta$-NiPc powder sample from binding energy ranging from $0 \mathrm{eV}$ to $1000 \mathrm{eV}$. This range covers all the core levels for the elements $\mathrm{N}, \mathrm{C}, \mathrm{Ni}, \mathrm{Fe}, \mathrm{Mn}$, and Co. XPS analysis indicates the impurity is Fe but with very small concentration (atomic concentration less than $0.2 \%$ ). However, it was difficult to exactly quantify the concentration because of the weakness of the impurity line. Since Fe oxidizes easily, it is likely that the impurity is really an oxide of iron such as ferrimagnetic magnetite $\left(\mathrm{Fe}_{3} \mathrm{O}_{4}\right)$ or maghemite $\left(\gamma-\mathrm{Fe}_{2} \mathrm{O}_{3}\right)$. 


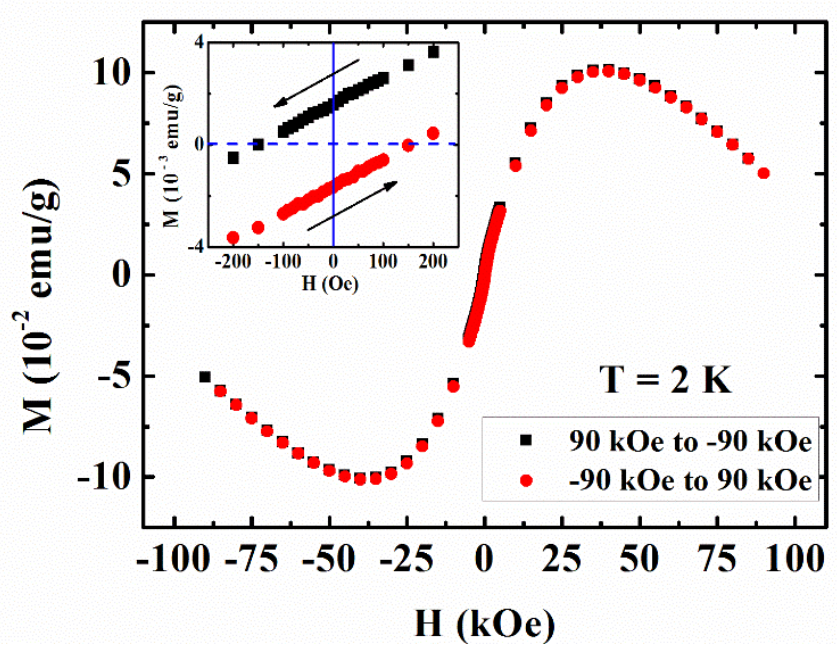

Fig. 7.8 Hysteresis loop measured between $+90 \mathrm{kOe}$ to $-90 \mathrm{kOe}$ at $2 \mathrm{~K}$ for $\beta$-NiPc. The inset is the zoom of the low-field region of the hysteresis loop at $2 \mathrm{~K}$.

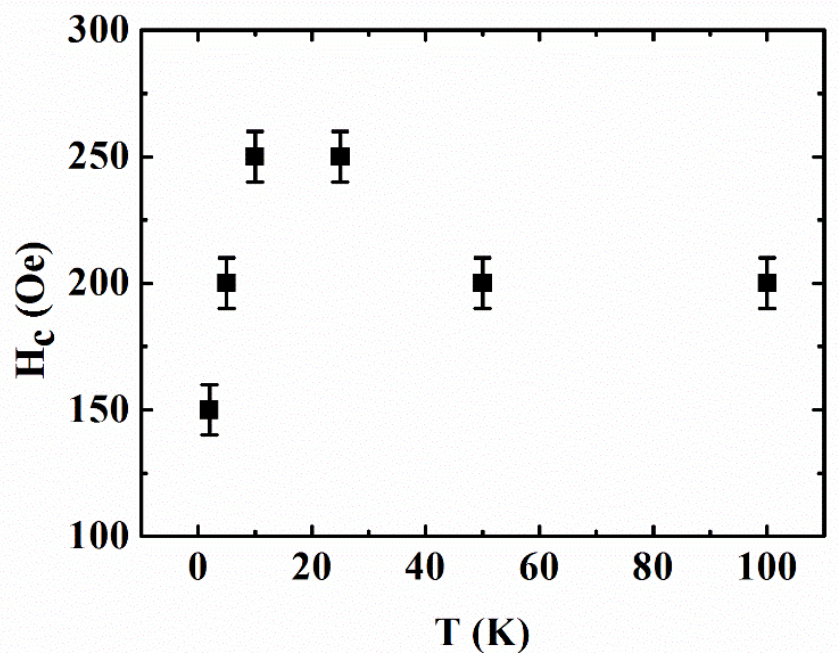

Fig. 7.9 Coercivity HC vs. temperature Tat $2 \mathrm{~K}, 5 \mathrm{~K}, 10 \mathrm{~K}, 25 \mathrm{~K}, 50 \mathrm{~K}$, and $100 \mathrm{~K}$ for $\beta$-NiPc.

\subsection{Conclusions}

The major conclusions from the results presented in this chapter are as follows: (i) The diamagnetic nature of $\beta$-NiPc was confirmed by detailed magnetization measurements and analysis, and the diamagnetic susceptibility $\chi_{\mathrm{D}}=-3.38 \times 10^{-7} \mathrm{emu} / \mathrm{gOe}$ is obtained. This is in agreement with the room 
temperature measurements of $\chi_{\mathrm{D}}$ reported for pure $\beta-\mathrm{NiPc}, \mathrm{H}_{2} \mathrm{Pc}$ and $\mathrm{ZnPc}[2]$; (2) The ferromagnetic component in the commercial sample of $\beta-\mathrm{NiPc}$ is due to Fe impurity with a concentration of less than $0.2 \%$. However it is highly unlikely that the impurity is Fe metal because Fe gets easily oxidized to its oxides. This concentration is too small to be detected in the standard x-ray diffraction experiments; (3) A procedure is developed to separate the magnetic contributions of pure NiPc from that of the ferromagnetic impurity. However the exact chemical nature of the impurity could not be determined from the techniques employed here.

\section{References}

[1] A.B.P. Lever, J. Chem. Soc. 1821 (1965).

[2] C.G. Barraclough, R.L. Martin and S. Mitra, J. Chem. Phys. 55, 1426 (1970).

[3] N. Marom and L. Kronik, Appl. Phys. A. 95, 159 (2009).

[4] Z. Hu, B. Li, A. Zhao, J. Yang and J. Hou, J. Phys. Chem. C. 112, 13650 (2008). 


\section{CHAPTER 8.}

\section{Magnetic Properties of $\beta$ - Manganese Phthalocyanine}

\subsection{Introduction}

In this chapter, detailed measurements and analysis of the temperature ( $2 \mathrm{~K}$ to $300 \mathrm{~K})$ and magnetic field (up to $90 \mathrm{kOe}$ ) dependence of the dc and ac magnetic susceptibilities in a powder sample of $\beta-\mathrm{MnPc}$ are presented. Previous magnetic studies in $\beta-\mathrm{MnPc}$ have reported it to be a canted ferromagnet below $\mathrm{T}_{\mathrm{C}} \approx 8.6 \mathrm{~K}$ [1-7]. However, the recent result of the lack of a $\lambda$-type anomaly in the specific heat vs. temperature data near the quoted $\mathrm{T}_{\mathrm{C}}$ has questioned the presence of long-range 3-dimensional (3D) magnetic ordering in this system [8,9]. Therefore, more detailed magnetic measurements and analysis was undertaken to determine the nature of magnetism and magnetic ordering in this system. Our magnetic measurements and analysis presented in this chapter leads to the conclusion that $3 \mathrm{D}$ long-range magnetic ordering is absent in $\beta$-MnPc. This is supported by the Arrott plots and the lack of a peak in the ac susceptibilities, $\chi^{\prime}$ and $\chi^{\prime \prime}$, near the quoted $\mathrm{T}_{\mathrm{C}}$. Instead, the system can be best described as an Ising-like chain magnet with Arrhenius relaxation of the magnetization governed by an intra-layer ferromagnetic exchange constant $\mathrm{J}_{\mathrm{ex}} / \mathrm{k}_{\mathrm{B}}=2.6 \mathrm{~K}$ and the single ion anisotropy energy parameter $|\mathrm{D}| / \mathrm{k}_{\mathrm{B}}=8.3 \mathrm{~K}$. The absence of $3 \mathrm{D}$ long range order is consistent with our result of the measured $|\mathrm{D}|>\mathrm{J}_{\mathrm{ex}}$ [10]. A major portion of the results presented in this chapter have been published by the author in a recent paper [11]. 


\subsection{Sample and its Structural Characterization}

The powder sample of MnPc was purchased from Sigma Aldrich (stock \# 379557) and used as received. The x-ray diffraction (XRD) pattern of the sample performed using a PANAnalytical X'Pert Pro $\mathrm{X}$-ray diffractmeter $(\mathrm{Cu}-\mathrm{K} \alpha$ radiation, $\lambda=0.15148 \mathrm{~nm})$ is shown in Fig. 8.1. The XRD pattern matches perfectly with that expected pattern for $\beta$-MnPc (ICDD \#02-063-3894) having space group $\mathrm{P} 2{ }_{1} / \mathrm{c}$ and two molecules per unit cell in the monoclinic structure with lattice parameters: $a=1.4576 \mathrm{~nm}, \mathrm{~b}=0.4755 \mathrm{~nm}$, $\mathrm{c}=1.9362 \mathrm{~nm}$ and $\beta=120.7^{\circ}$. This structure is shown in Fig. 8.2. It is important to note that the pattern given in the ICDD card for $\beta$-MnPc is based on calculations and single crystal XRD data of Mason et al [12]. In the previous studies reported on $\beta-\mathrm{MnPc}$ [2-10] XRD patterns of the powder samples of $\beta$-MnPc were not reported to the best of our knowledge. The other relevant information from Fig. 8.2 is the distance $\mathrm{b}=0.4755 \mathrm{~nm}$ between the $\mathrm{Mn}$ ions in a chain and the stack angle $\delta=45^{\circ}$ representing orientation of the molecular planes with respect to the b-axis. The $\mathrm{Mn}^{2+}-\mathrm{Mn}^{2+}$ exchange coupling may result from both superexchange coupling through $\mathrm{N}$ atoms as well as direct exchange coupling. However why the overall $\mathrm{Mn}^{2+}-\mathrm{Mn}^{2+}$ exchange coupling in $\beta-\mathrm{MnPc}$ is ferromagnetic is not yet well understood [13-15]. 


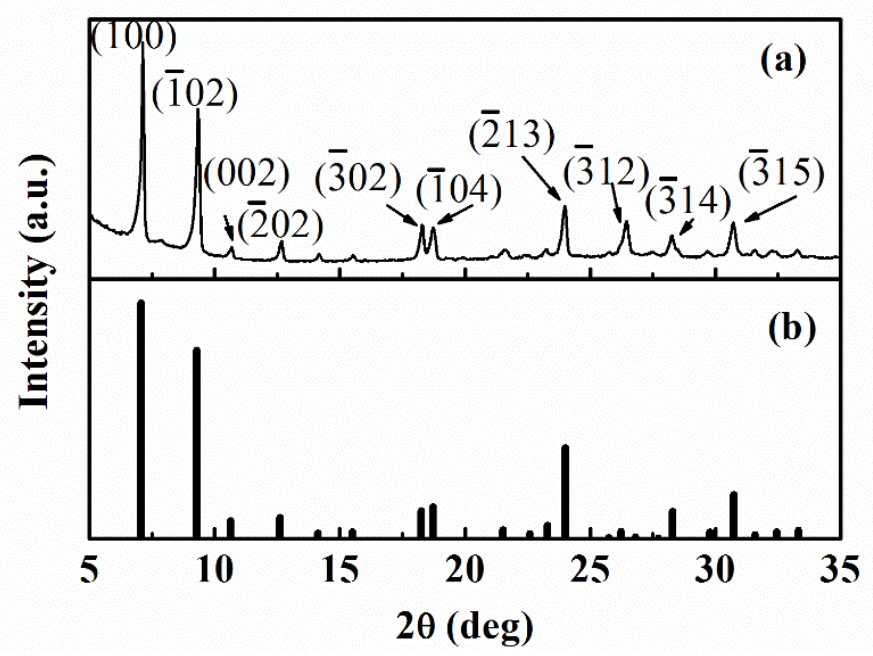

Fig. 8.1 (a) XRD pattern of the MnPc powder sample with Miller indices of major lines shown. The stick diagram in (b) shows the relative intensities of the lines according to the ICDD card (\#02-063-3894) for $\beta-M n P c$.

By measuring the width $\mathrm{w}$ of the major Bragg lines, the data was fit to the Williamson-Hall equation: $w \cos \theta=(0.9 \lambda / D)+\eta \sin \theta$ where $D$ is the crystallite size and $\eta$ is the strain. The straight line fit (Fig. 8.3) yielded $D=75(15) \mathrm{nm}$ for the crystallite size and $\eta=9(2) \times 10^{-3}$ for the strain. The morphology of particles of $\beta-\mathrm{MnPc}$ was examined by scanning electron microscopy (SEM) utilizing a JEOL7600 FE-SEM system at the DOE National Energy Technology Laboratory. The SEM micrograph of Fig. 8.4 shows plate-like morphology of the $\beta$-MnPc sample with 2 to $3 \mu \mathrm{m}$ sides and $50-100 \mathrm{~nm}$ thickness, the latter agreeing with the crystallite size of $\mathrm{D}=75(15) \mathrm{nm}$ determined from the analysis of the XRD data discussed above. 


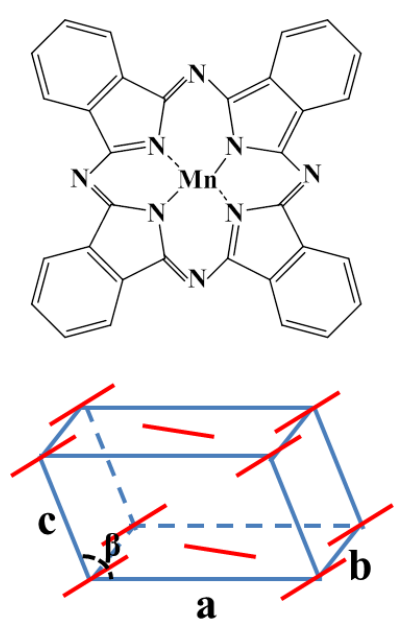

B-phase

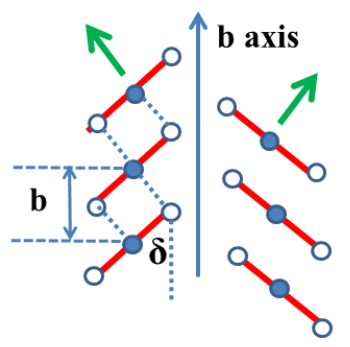

- Mn

○ $\mathbf{N}$

Fig. 8.2 Molecular model, unit cell, and molecular arrangement of $\beta-M n P c$. The red lines in the unit cell depict the orientation of the molecular planes of $\beta$-MnPc. The green arrows show the direction of magnetic moment perpendicular to the molecular plane, the discussion on which is given in the text.

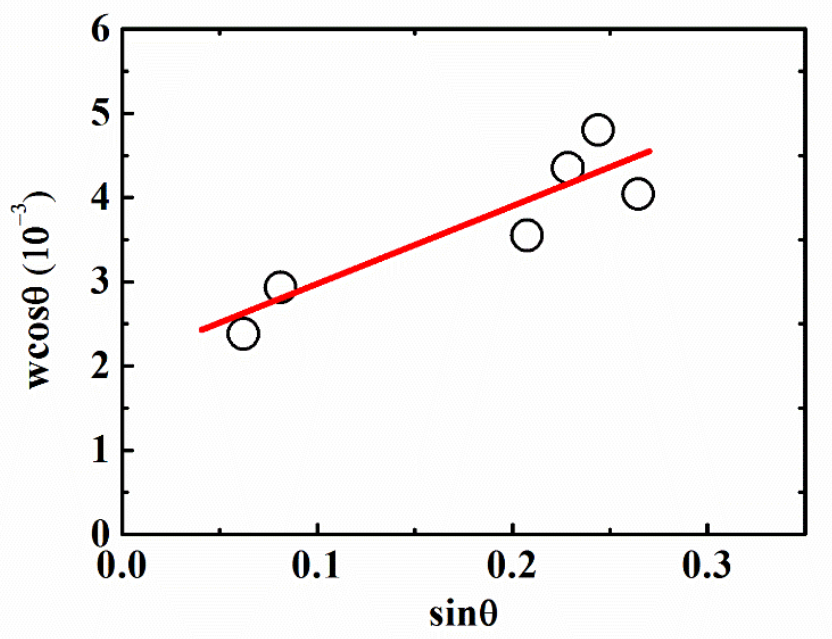

Fig. 8.3 The plot of $w \cos \theta$ versus $\sin \theta$ using prominent Bragg lines of $\beta$-MnPc to fit the Williamson-Hall equation: $w \cos \theta=(0.9 \lambda / D)+\eta \sin \theta$. 


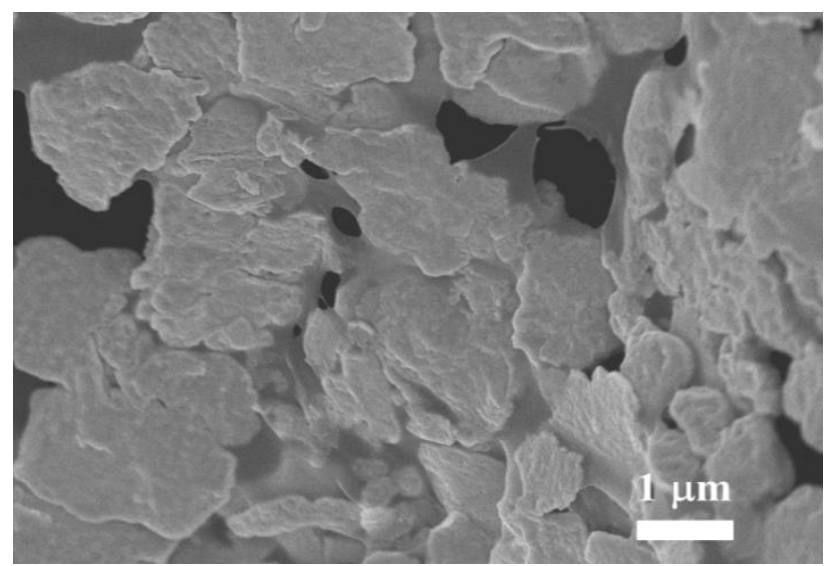

Fig. 8.4 A scanning electron micrograph for $\beta$-MnPc. The length of scale bar is $1 \mu \mathrm{m}$.

\subsection{Results from Magnetic Measurements, Analysis, and Discussion}

\subsubsection{Temperature Dependence of DC Magnetization and the Curie-Weiss Law}

The temperature dependence of the magnetic susceptibility $\chi=\mathrm{M} / \mathrm{H}$ measured in applied magnetic fields $\mathrm{H}=50 \mathrm{Oe}, 200 \mathrm{Oe}$ and $1 \mathrm{kOe}$ is shown in Fig. 8.5. A log-plot is used along the temperature axis to highlight the data at the lower temperatures. In the inset of Fig. 8.5, plot of $(\mathrm{d} \chi / \mathrm{dT})$ versus $\mathrm{T}$ for the $\mathrm{H}=1 \mathrm{kOe}$ data is shown yielding a peak near $9 \mathrm{~K}$. Similar analysis was used in earlier papers by several authors to infer a ferromagnetic $\mathrm{T}_{\mathrm{C}} \approx 8.6 \mathrm{~K}[1-7]$. 


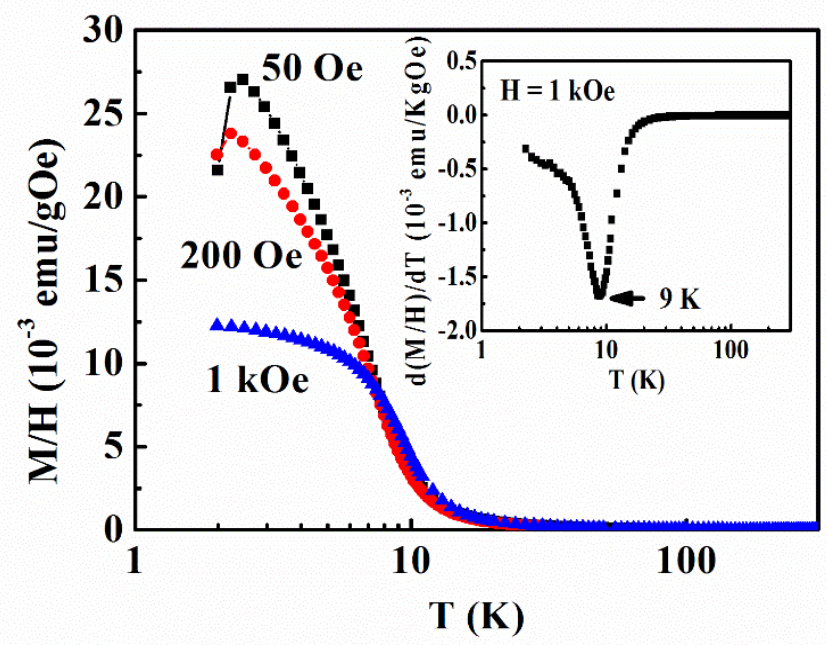

Fig. 8.5 Magnetic susceptibility $\chi=M / H$ vs. temperature for $\beta$-MnPc. The inset is the plot of $d \chi / d T$ versus $T$ data for the $H=1 \mathrm{kOe}$ data showing a peak at $9 \mathrm{~K}$.

The analysis of the data was done in a step-wise procedure as follows. First the data of $\chi$ vs. T from $2 \mathrm{~K}$ to $300 \mathrm{~K}$ was fitted to the modified Curie-Weiss (CW) law: $\chi=\chi_{0}+\mathrm{C} /(\mathrm{T}-\theta)$. For this purpose, $\chi_{0}=-4.83 \times 10^{-6} \mathrm{emu} / \mathrm{gOe}$ was determined by plotting $\chi$ vs. $1 / \mathrm{T}$ and linearly extrapolating the high temperature data to $1 / \mathrm{T} \rightarrow 0$, as shown in Fig. 8.6. This $\chi_{0}$ includes the diamagnetic contributions of the plastic sample holder and the sample itself. Next, by plotting $\left(\chi-\chi_{0}\right)^{-1}$ vs. T (see inset of Fig. 8.7), the linear fit yielded $\mathrm{C}=4.40 \times 10^{-3} \mathrm{emu}-\mathrm{K} / \mathrm{gOe}$ and $\theta=12.3 \mathrm{~K}$, the positive $\theta$ signifying ferromagnetic exchange coupling. Note that both $\beta$-CuPc and $\beta$-CoPc show antiferromagnetic exchange coupling between transition-metal ions whereas $\beta$-MnPc shows ferromagnetic exchange coupling although they have the similar crystal structure. In Fig. 8.7, the solid line A represents the fit to the modified CW law with the magnitudes of $\chi_{0}, \mathrm{C}$, and $\theta$ listed above. From Fig. 8.7, it is evident that the data of $\chi$ vs. T deviates strongly from the $\mathrm{CW}$ fit below about $15 \mathrm{~K}$. 


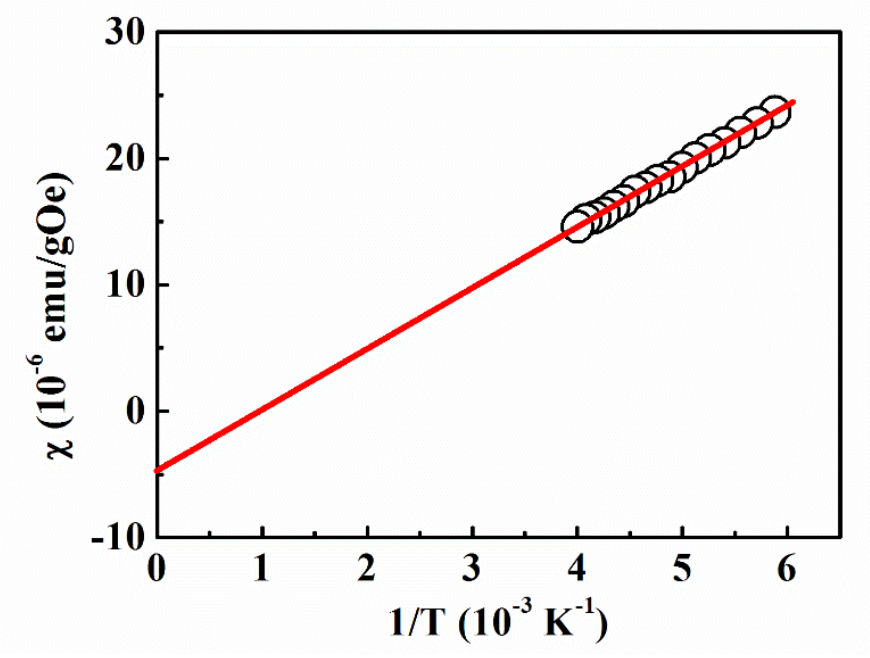

Fig. 8.6 Plot of magnetic susceptibility $\chi$ vs. 1/T for $\beta$-CoPc with $H=50$ Oe. The solid line is the linear fit of the experimental data and its interception with y axis $(\chi)$ gives $\chi_{o}=-4.83 \times 10^{-6}$ emu/gOe.

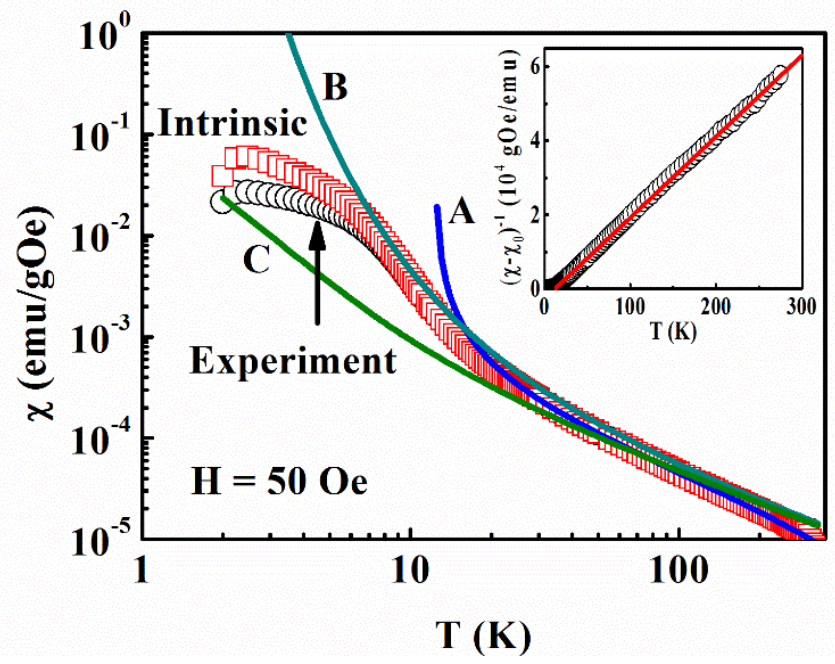

Fig. 8.7 Magnetic susceptibility $\chi$ vs. $T$ with $H=50$ Oe for $\beta$-MnPc where the "intrinsic" represents data corrected for demagnetizing factors. Solid line $A$ and the inset are fits to the modified Curie-Weiss law with $\chi_{o}=-4.83 \times 10^{-6} \mathrm{emu} / \mathrm{gOe}, \mathrm{C}=4.40 \times 10^{-3} \mathrm{emu}-\mathrm{K} / \mathrm{gOe}$, and $\theta=12.3 \mathrm{~K}$. Solid line $B(\mathrm{C})$ is fit to the Ising (Heisenberg) linear chain model with exchange constant $J_{e x} / k_{B}=2.6 \mathrm{~K}$.

Next $C=N \mu^{2} / 3 k_{B}$ where $N=N_{A} / M W$ is the number of $\mathrm{Mn}^{2+}$ ions per gm, $\mathrm{N}_{\mathrm{A}}$ is the Avogadro's number and $\mathrm{MW}=567.46 \mathrm{~g} / \mathrm{mol}$ is the molecular weight of $\beta-\mathrm{MnPc}$, was used yielding $\mu=4.467 \mu_{\mathrm{B}}$ as 
the magnetic moment per $\mathrm{Mn}^{2+}$ ion. Also using $\mu^{2}=\mathrm{g}^{2} \mathrm{~S}(\mathrm{~S}+1)$ yields $\mathrm{g}=2.307$ for $\mathrm{S}=3 / 2$. If $\mathrm{Mn}^{2+}$ in $\beta$-MnPc were in the usual high spin state with $S=5 / 2$, then $\mu=5.916 \mu_{\mathrm{B}}$ would have been observed with $\mathrm{g}=2$. Note that $\mathrm{g}=2.307$ is different from the free electron value of 2.00 because of the contribution from the partially restored orbital moment via spin-orbit coupling as in $\mathrm{CuPc}$ and $\mathrm{CoPc}$ systems. The g-shift is positive since for the $3 \mathrm{~d}^{5}$ state of $\mathrm{Mn}^{2+}$, the shell is more than half-full as discussed in Chapter II. The magnitude of $\mu=4.467 \mu_{\mathrm{B}}$ is in good agreement with the earlier data of Ref. 1 to 5 . The electronic configuration of the ground state is likely $\left[\left(\mathrm{d}_{\mathrm{XZ}, \mathrm{YZ}}\right)^{3}\left(\mathrm{~d}_{\mathrm{XY}}\right)^{1}\left(\mathrm{~d}_{\mathrm{Z}}{ }^{2}\right)^{1}\right]$ or $\left[\left(\mathrm{d}_{\mathrm{XZ}, \mathrm{YZ}}\right)^{2}\left(\mathrm{~d}_{\mathrm{XY}}\right)^{2}\left(\mathrm{~d}_{\mathrm{Z}}{ }^{2}\right)^{1}\right]$ as suggested by Brumboiu et al. [16].

\subsubsection{Temperature Dependence of DC Magnetization, Heisenberg Linear Chain, and Ising Linear}

\section{Chain Analysis}

The positive $\theta=12.3 \mathrm{~K}$ suggests ferromagnetic coupling between $\mathrm{Mn}^{2+}$ ions as also noted by earlier investigators [1-9]. Since $\chi$ vs. T in Fig. 8.7 does not fit the $\mathrm{CW}$ law below about $15 \mathrm{~K}$, we attempted fits of the data to the predictions of both the Heisenberg linear chain following our analysis in $\beta$-CoPc [17] and in $\alpha-\mathrm{CuPc}$ and $\beta$-CuPc using the BF model $[18,19]$ and the Ising linear chain model $[8,9$, 20]. The magnetic susceptibility in both cases follows the Eq. (8.1):

$$
\chi=\frac{C}{T} \cdot \frac{(1-\Gamma)}{(1+\Gamma)}
$$

However $\Gamma=\operatorname{coth}(y)-(1 / y)$ for the Heisenberg linear chains and $\Gamma=\tanh (y)$ for the $\quad$ Ising linear chains. Here $y=2 \mathrm{~J}_{\mathrm{ex}} \mathrm{S}^{2} /\left(\mathrm{k}_{\mathrm{B}} \mathrm{T}\right)$ with $\mathrm{J}_{\mathrm{ex}}$ being the exchange constant in the Heisenberg Hamiltonian $\mathrm{H}=-2 \mathrm{~J}_{\mathrm{ex}} \Sigma \mathbf{S}_{\mathrm{i}}$. 
$\mathbf{S}_{\mathbf{i}+\mathbf{1}}$. For the Ising linear chain, Eq. (8.1) reduces to:

$$
\chi=\frac{\mathrm{C}}{\mathrm{T}} \exp \left(4 \mathrm{~J}_{\mathrm{ex}} \mathrm{S}^{2} / \mathrm{k}_{\mathrm{B}} \mathrm{T}\right)
$$

The fit shown by curve B in Fig. 8.7 represents Eq. (8.2) with $\mathrm{C}=4.40 \times 10^{-3} \mathrm{emu}-\mathrm{K} / \mathrm{gOe}$ (determined earlier from the $\mathrm{CW}$ fit above $15 \mathrm{~K}$ ) and $\mathrm{J}_{\mathrm{ex}} / \mathrm{k}_{\mathrm{B}}=2.6 \mathrm{~K}$ determined from the fit. Curve $\mathrm{B}$ fits the data well down to about $6 \mathrm{~K}$ and represents a major improvement over the $\mathrm{CW}$ fit. The data points marked "intrinsic" represent the corrected intrinsic susceptibility $\chi_{\mathrm{g}}(\mathrm{i})=\chi_{\mathrm{g}} /\left(1-4 \pi \rho \chi_{\mathrm{g}}\right)$ where $\chi_{\mathrm{g}}$ is the measured gram susceptibility and $\rho=1.61 \mathrm{~g} / \mathrm{cm}^{3}$ is the density of $\beta$-MnPc. It is noted that $\chi_{\mathrm{g}}(\mathrm{i})$ takes into account the demagnetization factor of $4 \pi$ valid for a thin plate with $\mathrm{H}$ perpendicular to the plate. Although $\chi_{\mathrm{g}}(\mathrm{i})>\chi_{\mathrm{g}}$ for $\mathrm{T}<6 \mathrm{~K}$, it is still less than the magnitude expected for $\chi$ from Eq. (8.2) for $\mathrm{T}<6 \mathrm{~K}$. As shown by curve $\mathrm{C}$, the data does not fit the predictions of Heisenberg linear chain very well unlike the case for $\beta$-CuPc with AFM exchange interaction [19]. The difference between these cases likely result from the larger anisotropy in $\beta$-MnPc as reflected in the larger value of $\mathrm{g}=2.307$ compared to $\mathrm{g}=2.10$ in $\beta$-CuPc [19].

According to Eq. (8.2), a plot of $\ln (\chi \mathrm{T} / \mathrm{C})$ against $1 / \mathrm{T}$ should yield a straight line. This plot is shown in Fig. 7.8 showing a near linear variation for $T>6 \mathrm{~K}$ with the slope $=4 \mathrm{~S}^{2} \mathrm{~J}_{\mathrm{ex}} / \mathrm{k}_{\mathrm{B}}$ yielding $\mathrm{J}_{\mathrm{ex}} / \mathrm{k}_{\mathrm{B}}=$ 2.6(0.2) K. The deviations from Eq. (8.2) observed in Figs. 7.7 and 7.8 for $\mathrm{T}<6 \mathrm{~K}$ likely result from the fact that the normalized magnetic correlation length $\xi / \mathrm{a}=\chi \mathrm{T} / 2 \mathrm{C}(\mathrm{a}=$ cell parameter of the chain) remains finite for the Ising model in the limit $\mathrm{T}=0 \mathrm{~K}$ [20] because of the energy gap $\Delta / \mathrm{k}_{\mathrm{B}}=65.5 \mathrm{~K}$ determined later. This energy gap leads to the formation of domains separated by sharp domain walls, thus keeping 
correlation length finite in the limit $\mathrm{T}=0 \mathrm{~K}$ [20]. From the plot in Fig. 8.8, near $6 \mathrm{~K}$ the normalized correlation length $\approx \mathrm{e}^{3} / 2 \approx 10$, defining the size of the domains along the chain length. A second possible reason for the departure of the data from the prediction of the Ising model is that our measurements are on a powder sample and hence the measured $\chi$ contains both the parallel and perpendicular susceptibilities, the latter remaining finite for the Ising model even in the limit of $\mathrm{T}=0 \mathrm{~K}[4,21]$.

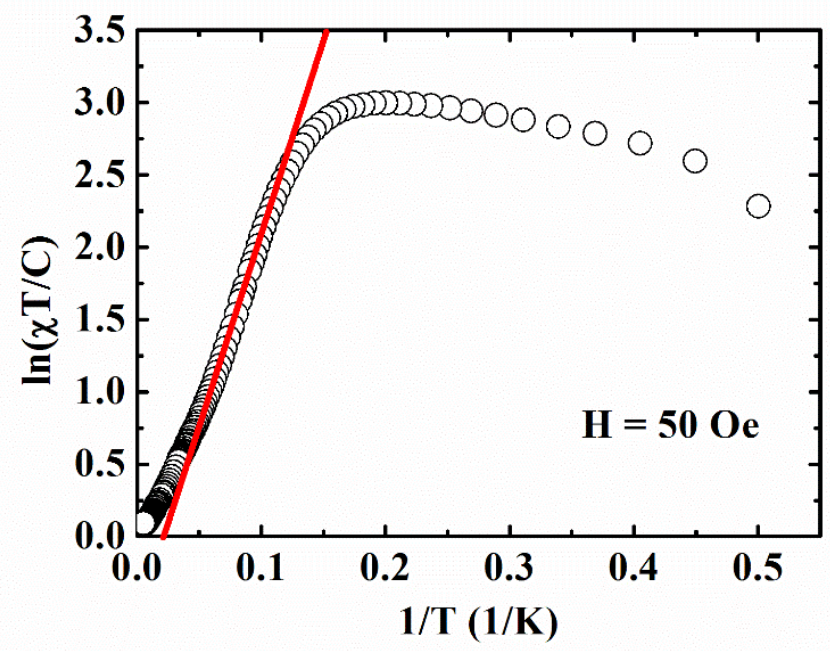

Fig. $8.8 \ln (\chi T / C)$ vs. $1 / T$ for $\beta$-MnPc. The solid line is the linear fit to Eq. (8.2) with $J_{e x} / k_{B}=2.6 \mathrm{~K}$.

\subsubsection{Magnetic Field Dependence of Magnetization and Ising Linear Chain Analysis}

The magnetic field dependence of the magnetization at $2 \mathrm{~K}$ was next fit to the prediction of the Ising linear chain model. The normalized magnetic field dependence of the magnetization $\mathrm{M}$ measured at $2 \mathrm{~K}$ for the powder sample of $\beta-\mathrm{MnPc}$ is shown as curve $\mathrm{C}$ in Fig. 8.9 using $\mathrm{M}_{\mathrm{S}}=31 \mathrm{emu} / \mathrm{g}$ determined in the limit of $1 / \mathrm{H} \rightarrow 0$ (see inset of Fig. 8.9). The magnetization does not quite saturate even at $90 \mathrm{kOe}$. The hysteresis loop of $\beta$-MnPc was measured at $2 \mathrm{~K}$ between $\mathrm{H}=+90 \mathrm{kOe}$ and $-90 \mathrm{kOe}$ with the zoom of the low-field region shown in the other inset of Fig. 8.9. There is practically no hysteresis within the 
experimental uncertainty of about 20 Oe for setting the field to zero. The absence of hysteresis is consistent with the absence of a long-range ferromagnetic ordering.

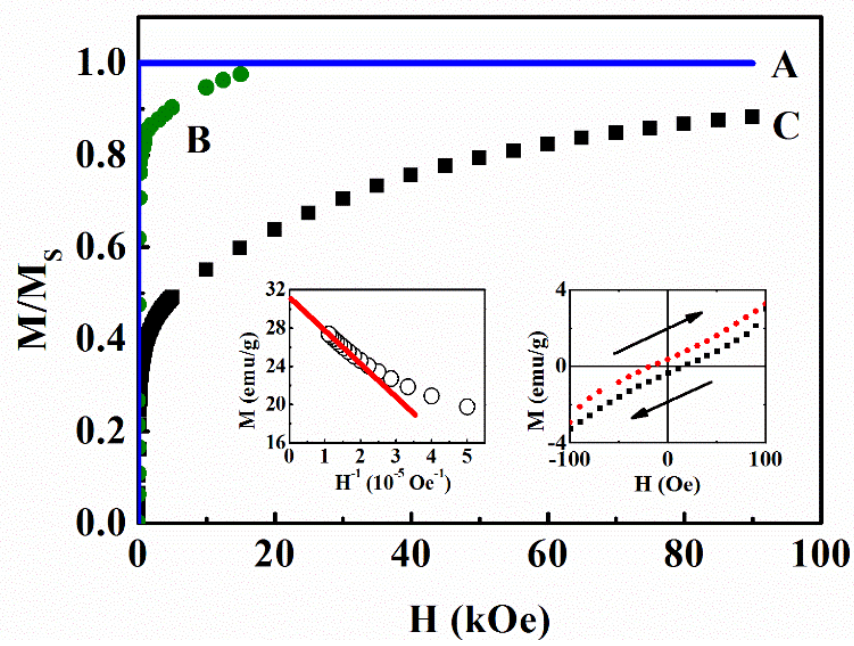

Fig. 8.9 Solid line A is the calculated curve using Eq. (8.3). Curve C represent our experimental data taken at $2 \mathrm{~K}$ on the powder sample of $\beta-M n P c$ and normalized to $M_{S}=31 \mathrm{emu} / \mathrm{g}$ determined in the limit $1 / H \rightarrow O$ (left inset). Data represented by curve B is the experimental data of $M v s . H$ at $4.2 \mathrm{~K}$ for single crystal of $\beta-M n P c$ along the easy axis and taken from the paper by Mitra et al. [5].The right inset is the zoom of the low-field region of the hysteresis loop.

The $\mathrm{M}$ vs $\mathrm{H}$ variation for Ising-linear chain magnet is predicted to follow the equation [20]:

$$
\frac{\mathrm{M}}{\mathrm{M}_{\mathrm{S}}}=\frac{\sinh \left(\mu \mathrm{H} / \mathrm{k}_{\mathrm{B}} \mathrm{T}\right)}{\sqrt{\sinh ^{2}\left(\mu \mathrm{H} / \mathrm{k}_{\mathrm{B}} \mathrm{T}\right)+\exp \left(-8 \mathrm{~J}_{\mathrm{ex}} \mathrm{S}^{2} / \mathrm{k}_{\mathrm{B}} \mathrm{T}\right)}}
$$

The solid line A shown in Fig. 8.9 is the calculated curve using Eq. (8.3) for $\mathrm{T}=2 \mathrm{~K}$ and $\mu=4.467 \mu_{\mathrm{B}}$, $\mathrm{J}_{\mathrm{ex}} / \mathrm{k}_{\mathrm{B}}=2.6 \mathrm{~K}$ and $\mathrm{S}=3 / 2$ evaluated earlier for $\beta$-MnPc. This curve saturates at very low magnetic fields unlike our experimental data for the powder sample of $\beta$-MnPc. The data shown in curve $\mathrm{B}$ is the experimental data taken from the paper by Mitra et al [5] which was measured at $4.2 \mathrm{~K}$ in single crystal of 
$\beta$-MnPc with $\mathrm{H}$ up to $15 \mathrm{kOe}$ applied along the easy axis; it reaches near-saturation at around $3 \mathrm{kOe}$ closer to the predicted variation of Eq. (8.3). Note that the data in curve B was reproduced from the original paper [5] using the internet-available software "digitizer". This software allows one to take a scanned image of a plot and digitize values off the plot by clicking the mouse on each data point. The data was then saved and replotted. Both curves $\mathrm{B}$ and $\mathrm{C}$ show rapid rise in $\mathrm{M}$ at very low $\mathrm{H}$ followed by a gradual increase at the higher $\mathrm{H}$. For the powder sample, the lack of rapid saturation even up to $90 \mathrm{kOe}$ is likely due to the contributions from the afore-mentioned perpendicular susceptibility [4, 21]. The domains canted relative to the b-axis [5, 7] are the likely reason for this slow rate of saturation of the magnetization. This domain structure taken from the paper by Awaga et al. [6] is shown in Fig. 8.10.

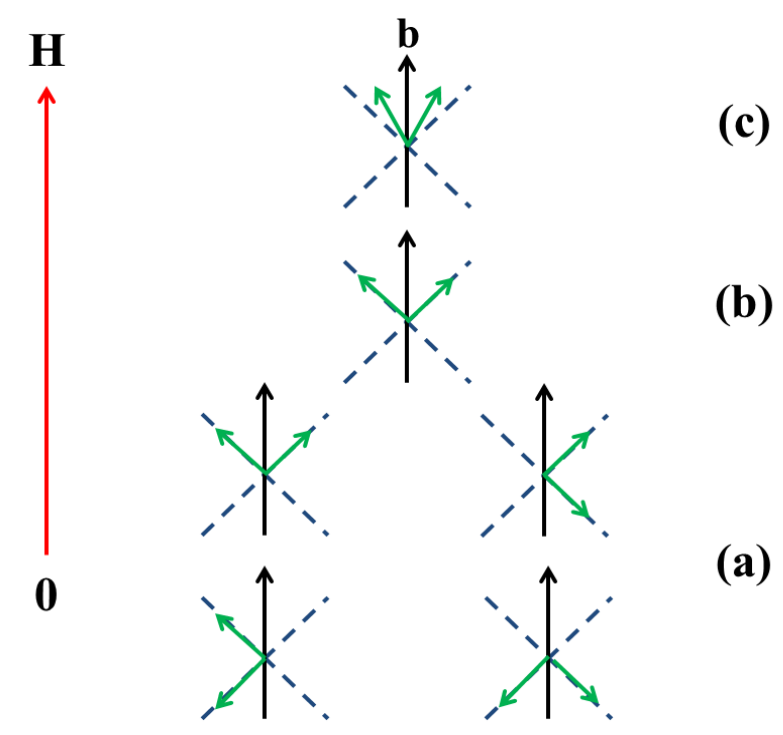

Fig. 8.10 Domain structure in $\beta$-MnPc reproduced from the paper by Awaga et al. [6]. The applied magnetic field is along the b-axis (black arrow), the two green arrows show the moments on the neighboring chains. (a) Four kinds of magnetic domains at zero field. (b) Single domain under the magnetic field. (c) Rotation of the magnetic moments toward the magnetic field. 

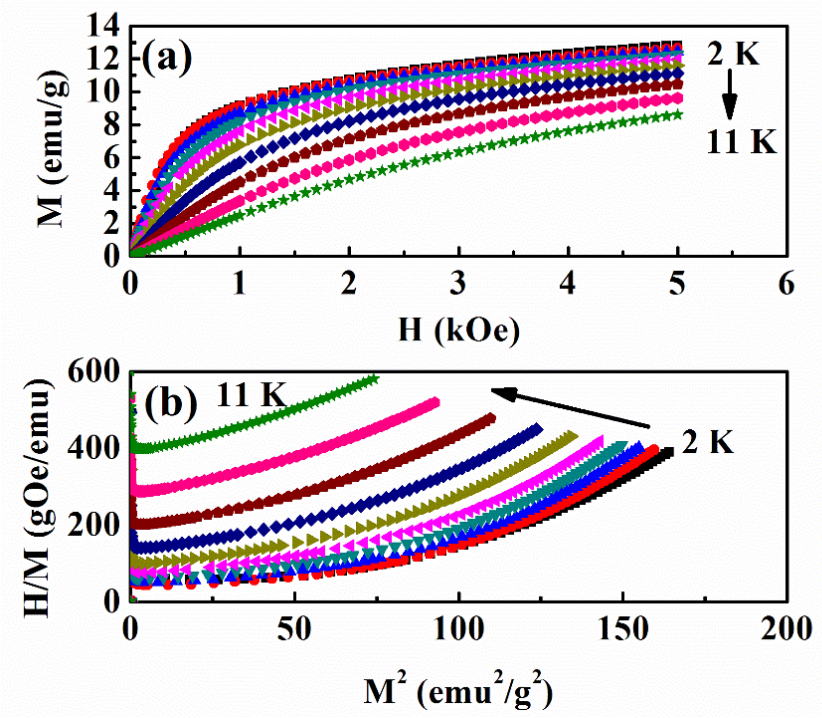

Fig. 8.11(a) Magnetization Mvs. magnetic field $H$ for $\beta$-MnPc measured in $1 \mathrm{~K}$ intervals between $2 \mathrm{~K}$ and $11 \mathrm{~K}$; (b) Data of (a) plotted as H/M vs. $M^{2}$.

\subsubsection{Arrott Plots}

According to the Landau theory of phase transitions [22], near $\mathrm{T}_{\mathrm{C}}$ the magnetization $\mathrm{M}$ in a magnetic field $\mathrm{H}$ should follow the equation: $\mathrm{MH}=\mathrm{a}\left(\mathrm{T}-\mathrm{T}_{\mathrm{C}}\right) \mathrm{M}^{2}+\mathrm{bM}^{4}$ where $\mathrm{a}$ and $\mathrm{b}$ temperature-independent constants. This equation can be rearranged as:

$$
\frac{\mathrm{H}}{\mathrm{M}}=\mathrm{bM}^{2}+\mathrm{a}\left(\mathrm{T}-\mathrm{T}_{\mathrm{C}}\right)
$$

Therefore, plots of H/M vs. $\mathrm{M}^{2}$ at different temperatures bracketing the expected $\mathrm{T}_{\mathrm{C}}$ should have a positive or negative intercept depending on whether $\mathrm{T}>\mathrm{T}_{\mathrm{C}}$ or $\mathrm{T}<\mathrm{T}_{\mathrm{C}}$. At $\mathrm{T}=\mathrm{T}_{\mathrm{C}}$, the plot should go through the origin. These are called the Arrott plots and they present a way of accurately locating the $\mathrm{T}_{\mathrm{C}}$ of a ferromagnet [23-25]. For this purpose, $\mathrm{M}$ vs. $\mathrm{H}$ was measured for $\mathrm{H}$ up to $5 \mathrm{kOe}$ at one-degree temperature interval between $2 \mathrm{~K}$ and $11 \mathrm{~K}$ bracketing the presumed $\mathrm{T}_{\mathrm{C}}$ near $9 \mathrm{~K}$ and the data are shown in Fig. 8.11 (a). The Arrott plots resulting from this data are shown in Fig. 8.11 (b). None of the plots intersect the $\mathrm{M}^{2}$ axis at any temperature ruling out traditional $\mathrm{T}_{\mathrm{C}}$. However, using a linear extrapolation 
from the higher $\mathrm{M}^{2}$ values to zero, a case can be made for $\mathrm{T}_{\mathrm{C}} \approx 9 \mathrm{~K}$ as done in the earlier paper by Miyoshi et al [3-4]. However, validity of such extrapolation is questionable in view of the data presented in Fig. 8.11.

\subsubsection{Temperature Dependence of Specific Heat}

A case against $3 \mathrm{D}$ ordering in $\beta-\mathrm{MnPc}$ with a ferromagnetic $\mathrm{T}_{\mathrm{C}}$ can also be made from the published data of the temperature dependence of heat capacity $\mathrm{C}$ by Taguchi et al [8-9]. Again, using the web-accessed software "Digitizer", this data was digitized and it is re-plotted in Fig. 8.12. There is no $\lambda$-type anomaly in the $\mathrm{C}$ vs. $\mathrm{T}$; instead there is a broad peak with the center at around $12 \mathrm{~K}$. This result also rules out the traditional long-range 3D order in $\beta-\mathrm{MnPc}$ since in case of a second order transitions, a $\lambda$-type peak in the $\mathrm{C}$ vs T. data should be observed [26]. It is also noted that in the neutron diffraction studies of $\beta-\mathrm{MnPc}$ carried out $5 \mathrm{~K}$ by Figgis et al [27], "no clear evidence for any magnetic contribution to the Bragg intensities" could by convincingly found.

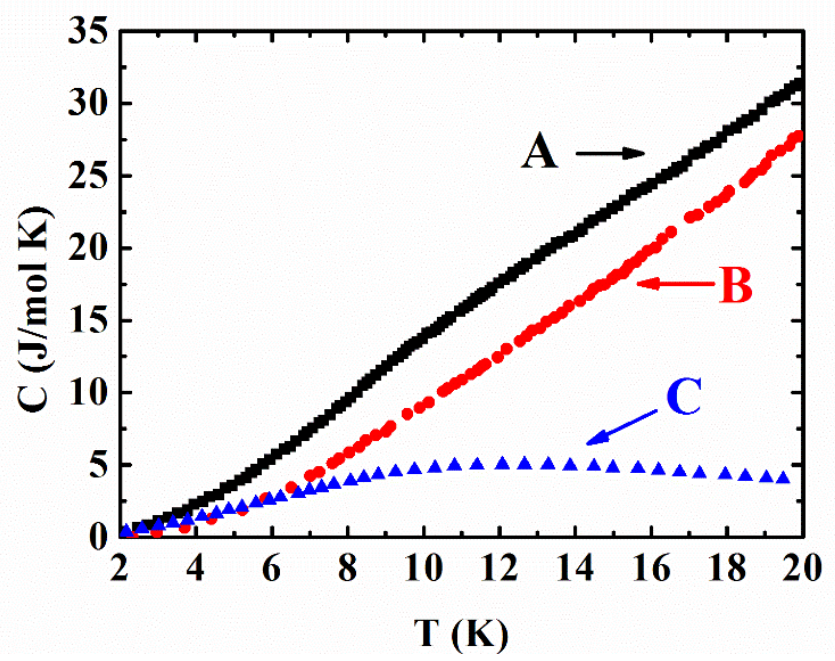

Fig. 8.12 Temperature dependence of total heat capacity (A), estimated lattice contribution (B), and magnetic contribution to the heat capacity $(C)$ of pristine $\beta$-MnPc. The lattice contribution to the heat capacity was estimated from that of $\beta$-NiPc in which $\mathrm{Ni}^{2+}$ has non-magnetic configuration. 


\subsubsection{Temperature and Frequency Dependence of AC Susceptibilities}

The temperature dependence of ac susceptibilities, $\chi^{\prime}$ and $\chi^{\prime \prime}$, of $\beta-\mathrm{MnPc}$ was measured at six different frequencies namely $\mathrm{f}_{\mathrm{m}}=10,50,100,500,1000$, and $5000 \mathrm{~Hz}$ using $\mathrm{H}_{\mathrm{ac}}=5$ Oe and $\mathrm{H}_{\mathrm{dc}}=0$ Oe. The results in Fig. 8.13 clearly show a peak in $\chi^{\prime \prime}$ near $3 \mathrm{~K}$ which shifts to higher temperatures with increase in $\mathrm{f}_{\mathrm{m}}$. The temperature dependence of $\chi^{\prime}$ is such that the expected relation $\chi^{\prime \prime} \sim \partial\left(\chi^{\prime} \mathrm{T}\right) / \partial \mathrm{T}$ is satisfied $[28,29]$. Importantly there is no peak in either $\chi^{\prime}$ or $\chi^{\prime \prime}$ in the $8 \mathrm{~K}$ to $10 \mathrm{~K}$ range where earlier studies [1-7] had reported the location of $T_{C}$ from dc susceptibilities using analysis similar to that shown in the inset of Fig. 7.5. These results combined with a lack of $\lambda$-type anomaly in the specific heat provide convincing proof that the long-range $3 \mathrm{D}$ ordering is not present in $\beta-\mathrm{MnPc}$. We also measured the effect of non-zero $\mathrm{H}_{\mathrm{dc}}$ on $\chi^{\prime}$ and $\chi^{\prime \prime}$. The results for one frequency comparing the data for $\mathrm{H}_{\mathrm{dc}}=0$ Oe with that for $\mathrm{H}_{\mathrm{dc}}=200$ Oe are shown in Fig. 8.14. The log-plot is used for $\chi^{\prime \prime}$ to show measurable values of $\chi^{\prime \prime}$ beginning around $14 \mathrm{~K}$. However, the effect of non-zero dc fields is to shift the peak in $\chi^{\prime \prime}$ near $3 \mathrm{~K}$ only slightly to a lower temperature. 


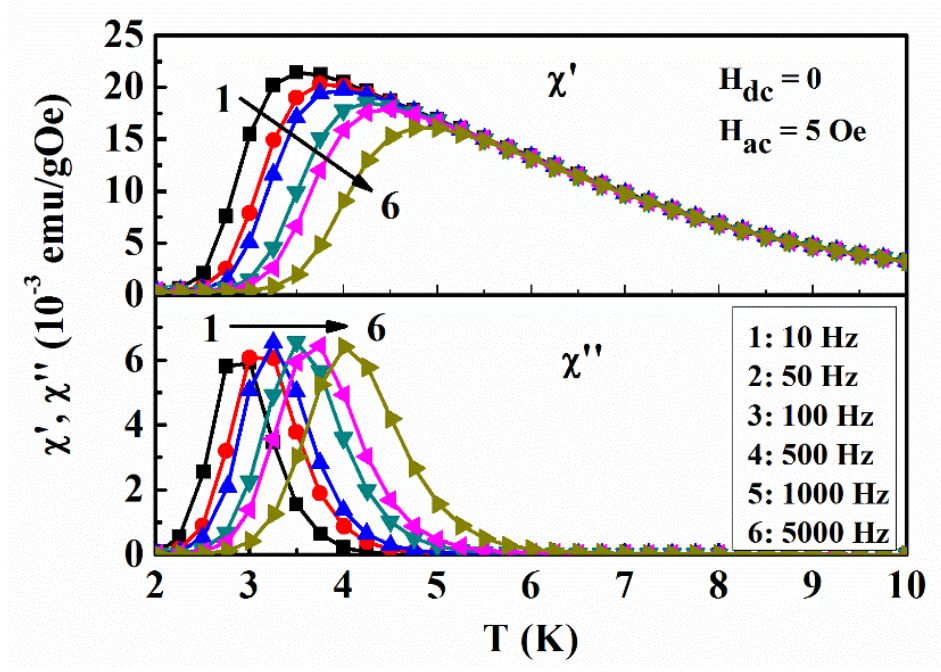

Fig. 8.13 Frequency dependence of the ac susceptibilities $\chi^{\prime}$ and $\chi^{\prime \prime}$ for $\beta$-MnPc measured in zero applied static field with $H_{a c}=5$ Oe at several frequencies between $10 \mathrm{~Hz}$ and $5000 \mathrm{~Hz}$.

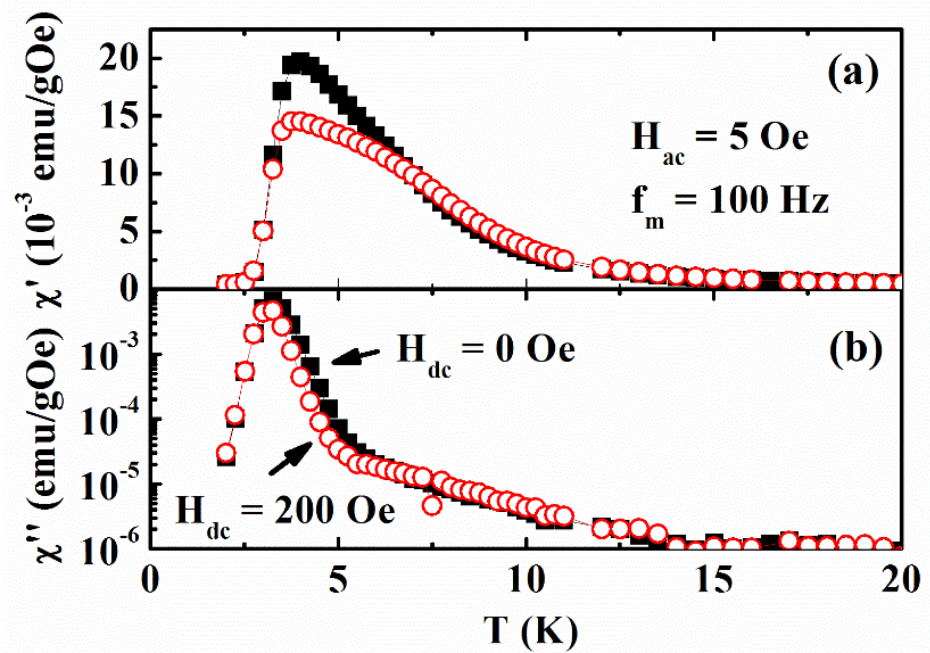

Fig. 8.14 Temperature dependence of the ac susceptibilities $\chi^{\prime}$ and $\chi^{\prime \prime}$ for $\beta$-MnPc in $H_{d c}=0$ Oe (a) and $H_{d c}=200 \mathrm{Oe}(\mathrm{b})$ with $H_{a c}=5$ Oe at frequency of $100 \mathrm{~Hz}$.

The shift in the peak position of $\chi$ " to higher temperatures with increase in $f_{m}$ is similar to the observations in magnetic nanoparticles [28-31] where the peak in $\chi^{\prime \prime}$ defines the blocking temperature $T_{B}$ given by: 


$$
\mathrm{T}_{\mathrm{B}}=\mathrm{T}_{\mathrm{o}}+\frac{\Delta}{\mathrm{k}_{\mathrm{B}} \ln \left(\mathrm{f}_{\mathrm{o}} / \mathrm{f}_{\mathrm{m}}\right)}
$$

Here $\Delta$ is the anisotropy energy defining the barrier to flipping of the magnetization, $f_{o}$ is the characteristic attempt frequency of the system and $T_{o}$ represents the strength of the interparticle interaction (IPI). Another parameter that defines IPI is the parameter $\Phi$ defined by [31]

$$
\Phi=\frac{\delta \mathrm{T}_{\mathrm{B}}}{\mathrm{T}_{\mathrm{B}} \delta\left(\log _{10} \mathrm{f}_{\mathrm{m}}\right)}
$$

In Eq. (8.6), $\delta \mathrm{T}_{B}$ represents change in $\mathrm{T}_{\mathrm{B}}$ with change $\delta\left(\log _{10} \mathrm{f}_{\mathrm{m}}\right)$ in the measurement frequency $\mathrm{f}_{\mathrm{m}}$. Usually $\Phi>0.13$ implies that IPI is negligible and hence $T_{0}=0$. For the result shown in Fig. 8.13 for $\beta$-MnPc, $\Phi=0.14$ is determined signifying negligible IPI and $\mathrm{T}_{\mathrm{o}}=0$. This value $\Phi=0.14$ rules out the possibility of spin-glass like ordering proposed by Taguchi et al. [8, 9] since for spin glasses $\Phi<0.05$ [31].

To determine $\Delta$ and $\mathrm{f}_{\mathrm{o}}$ for our system, $1 / \mathrm{T}_{\mathrm{B}}$ was plotted against $\operatorname{lnf}_{\mathrm{m}}$ with the slope yielding $\Delta / \mathrm{k}_{\mathrm{B}}$ and intercept yielding $f_{0}$. The results of the changes in $T_{B}$ with $\operatorname{lnf}_{m}$ are plotted in Fig. 8.15 with the solid lines fit to Eq. (8.5) with $\mathrm{T}_{\mathrm{o}}=0, \mathrm{f}_{\mathrm{o}}=5.9 \times 10^{10} \mathrm{~Hz}$ and $\Delta / \mathrm{k}_{\mathrm{B}}=65.5 \mathrm{~K}$. Taguchi et al. [8] also reported ac data on $\beta$-MnPc similar to our data shown in Fig. 8.13; however, they did not carry out any analysis of the data. I re-plotted the ac data of Ref. 8 using the software "Digitizer"; our analysis of that data is also shown in Fig. 8.15 yielding identical magnitude of $\mathrm{f}_{\mathrm{o}}$ but slightly larger $\Delta / \mathrm{k}_{\mathrm{B}}=69.4 \mathrm{~K}$.

The physical interpretation of the results from the analysis of $\chi^{\prime}$ and $\chi^{\prime \prime}$ presented above in $\beta$-MnPc is considered next. In the $\chi$ vs. T data of Fig. 8.7, the data was fitted to Eq. (8.2) valid for Ising chain magnet. According to Glauber's theory for one-dimensional Ising chain, the relaxation rate $f=1 / \tau$ 
follows the Eq. (8.7) [20, 32, 33]

$$
\mathrm{f}=\mathrm{f}_{\mathrm{o}} \exp \left(-\Delta / \mathrm{k}_{\mathrm{B}} \mathrm{T}\right)
$$

In Eq. (8.7), $\Delta=\left(8 \mathrm{~J}_{\mathrm{ex}}+|\mathrm{D}|\right) \mathrm{S}^{2}$ with $\mathrm{J}_{\mathrm{ex}}$ being the exchange interaction, $|\mathrm{D}|$ is the single ion anisotropy and $f_{o}$ is the characteristic frequency of the system. As the temperature is varied, then at a certain temperature $T_{B}$, $f$ becomes equal to the measuring frequency $f_{m}$ yielding

$$
\mathrm{T}_{\mathrm{B}}=\frac{\Delta}{\mathrm{k}_{\mathrm{B}} \ln \left(\mathrm{f}_{\mathrm{o}} / \mathrm{f}_{\mathrm{m}}\right)}
$$

Eq. (8.8) is equivalent to Eq. (8.5) with $\mathrm{T}_{\mathrm{o}}=0$.

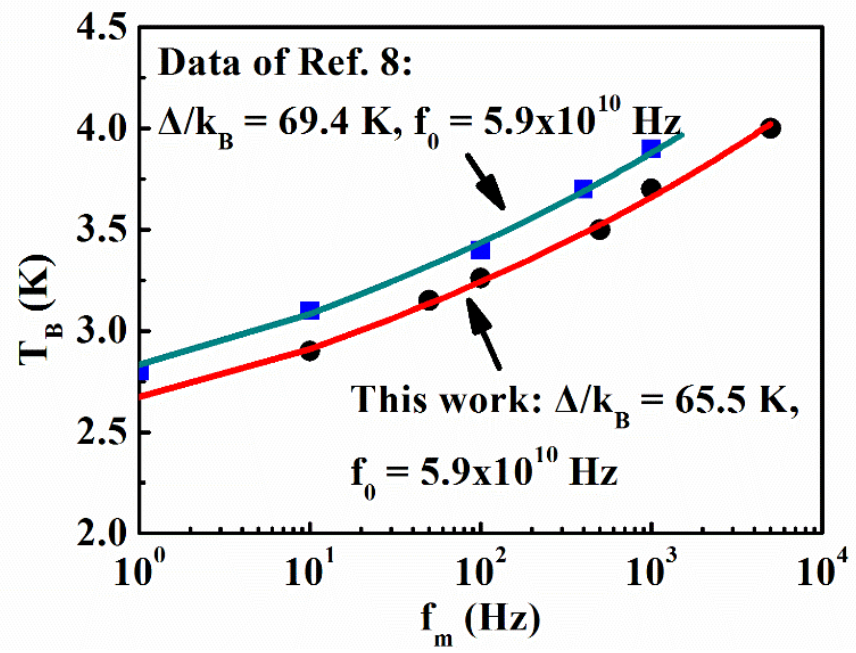

Fig. 8.15 Plot of the blocking temperature $T_{B}$ vs. fm with the solid curves fits to Eq. (8.8) with the parameters of the fits shown. The closed circles and squares are data points.

Using the the fits of the data in Fig. 8.15, $\Delta / \mathrm{k}_{\mathrm{B}}=65.5 \mathrm{~K}$ is determined. Substituting $\mathrm{J}_{\mathrm{ex}} / \mathrm{k}_{\mathrm{B}}=2.6$ $\mathrm{K}$ determined earlier (Fig. 8.7) in $\Delta=\left(8 \mathrm{~J}_{\mathrm{ex}}+|\mathrm{D}|\right) \mathrm{S}^{2}$ yields $|\mathrm{D}| / \mathrm{k}_{\mathrm{B}}=8.3 \mathrm{~K}$ as the magnitude of single ion anisotropy. This magnitude of $|\mathrm{D}| / \mathrm{k}_{\mathrm{B}}=8.3 \mathrm{~K}$ is considerably smaller than the theoretical estimate of $|\mathrm{D}| / \mathrm{k}_{\mathrm{B}}$ 
$=58 \mathrm{~K}$ given in Ref. 1 . However, $\mid \mathrm{D} / / \mathrm{k}_{\mathrm{B}}=8.3 \mathrm{~K}$ is still considerably larger than the magnitude of exchange constant $\mathrm{J}_{\mathrm{ex}} / \mathrm{k}_{\mathrm{B}}=2.6 \mathrm{~K}$. This is important because theoretically Moriya [10] has shown that for $|\mathrm{D}| / \mathrm{J}>1$, long range order is not possible and $\mathrm{T}_{\mathrm{C}}$ goes to zero. These results of the determination of $|\mathrm{D}| / \mathrm{k}_{\mathrm{B}}=8.3 \mathrm{~K}$ and $\mathrm{J}_{\mathrm{ex}} / \mathrm{k}_{\mathrm{B}}=2.6 \mathrm{~K}$ and establishing $|\mathrm{D}|>\mathrm{J}$ are important contributions of this work since they explain all the other experimental observations such as lack of $\lambda$-type anomaly, canting of the moments of the neighboring chains relative to the b-axis and absence of coercively at $2 \mathrm{~K}$. It is noted that using $\Delta / \mathrm{k}_{\mathrm{B}}=69.4 \mathrm{~K}$ determined from the fit of ac data of Taguchi et al. [8] in Fig. 7.15 yields slightly larger $|\mathrm{D}| / \mathrm{k}_{\mathrm{B}}=10 \mathrm{~K}$. However all the above arguments are still valid as long as $\mathrm{D}$ is larger than $\mathrm{J}_{\mathrm{ex}}$.

\subsubsection{Cole-Cole Analysis}

The data of Fig. 8.13 showing plots of $\chi^{\prime \prime}(\omega)$ and $\chi^{\prime}(\omega)$ vs. temperature measured at different frequencies are next anlyzed in terms of the Cole-Cole analysis [34]. The Cole-Cole equations for the complext susceptibility $\chi(\omega)=\chi^{\prime}(\omega)-\mathrm{i} \chi^{\prime \prime}(\omega)$ at a single temperature are given by [34]:

$$
\begin{gathered}
\chi^{\prime}(\omega)=\chi_{S}+\frac{\left(\chi_{0}-\chi_{S}\right)\left[1+(\omega \tau)^{1-\alpha} \sin (\alpha \pi / 2)\right]}{1+2(\omega \tau)^{1-\alpha} \sin (\alpha \pi / 2)+(\omega \tau)^{2(1-\alpha)}} \\
\chi^{\prime \prime}(\omega)=\frac{\left.\left(\chi_{0}-\chi_{S}\right)(\omega \tau)^{1-\alpha} \cos (\alpha \pi / 2)\right]}{1+2(\omega \tau)^{1-\alpha} \sin (\alpha \pi / 2)+(\omega \tau)^{2(1-\alpha)}}
\end{gathered}
$$

Here $\chi_{0}\left(\chi_{S}\right)$ is the isothermal (adiabatic) susceptibility in the limit of lowest (highest) frequency, $\tau$ is the relaxation time at the frequency $f=\omega / 2 \pi$ and $\alpha$ is a measure of the distribution of relaxation times. For $\alpha$ $=0$, Eqs. (8.9) and (8.10) reduce to equations characteristic of a single relaxation time (Debye process).

For $\alpha=1, \chi^{\prime \prime}(\omega)=0$ and $\chi^{\prime}(\omega)=\left(\chi_{0}+\chi_{S}\right) / 2$. For spin-glasses with a wide distribution of relaxation times, $\alpha$ approaching 0.9 may be observed [35]. The Cole-Cole analysis involves plotting $\chi^{\prime \prime}(\omega)$ vs. $\chi^{\prime}(\omega)$ 
at a fixed temperature and fitting it to the following equation derived from Eq. (8.9) and (8.10) [36]:

$$
\chi^{\prime \prime}\left(\chi^{\prime}\right)=-\frac{\chi_{0}-\chi_{S}}{2 \tan [(1-\alpha) \pi / 2]}+\left\{\left(\chi^{\prime}-\chi_{S}\right)\left(\chi_{0}-\chi^{\prime}\right)+\frac{\left(\chi_{0}-\chi_{S}\right)^{2}}{4 \tan ^{2}[(1-\alpha) \pi / 2]}\right\}
$$

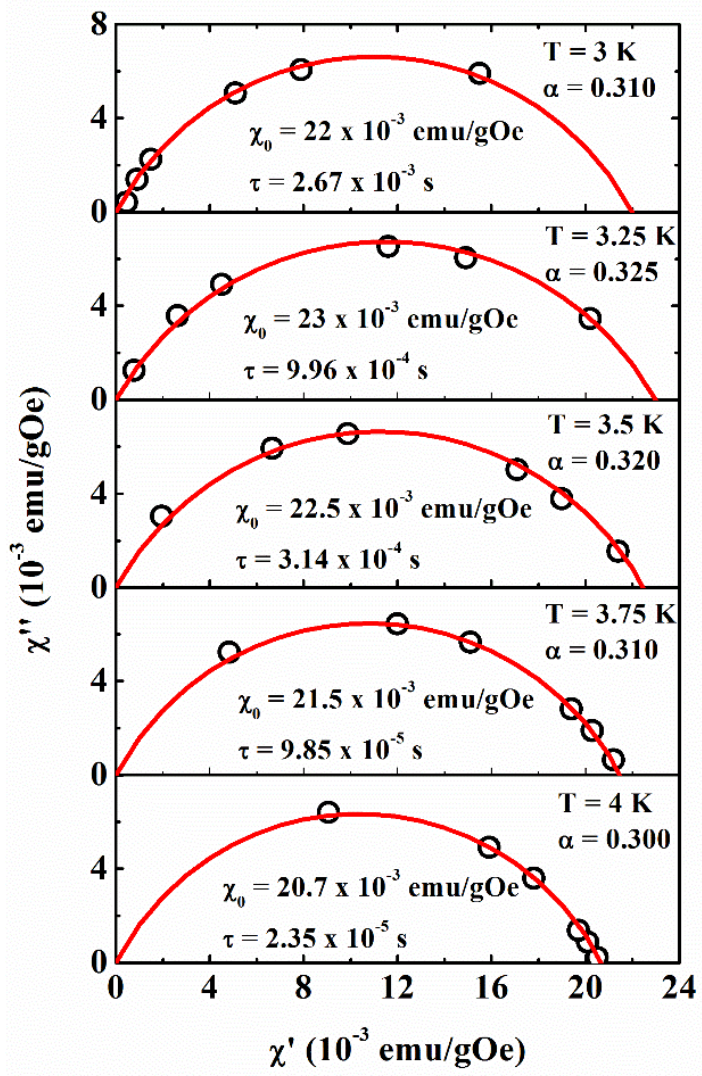

Fig. 8.16 Cole-Cole diagram at various temperatures from $3 \mathrm{~K}$ to $4 \mathrm{~K}$ for $\beta$-MnPc. The solid lines represent least-squares fits to Eq. (8.11) with the magnitudes of the various parameters determined from the fits listed in the figure.

For $\alpha=0$, the plot of $\chi^{\prime \prime}$ vs. $\chi^{\prime}$ is a semicircle. For $\alpha>0$, the plot is an arc which becomes increasingly compressed as $\alpha$ increases such that for $\alpha=1$, the plot is a straight line corresponding to $\chi^{\prime \prime}=0$. For $\beta$-MnPc, the Cole-Cole plots are shown in Fig. 8.16 for five temperatures between $3 \mathrm{~K}$ and $4 \mathrm{~K}$ with the solid lines 
as fits to Eq. (8.11) using $\chi_{S}=0$. The magnitude of $\alpha=0.31 \pm 0.01$ signifies that there is some distribution in the relaxation time although the system is far from being a spin-glass. This distribution likely results from distribution in the length of domains along the chain length in $\beta$-MnPc as noted earlier.

The mean relaxation time $\tau$ at each temperature T was next determined from the equation for $\chi^{\prime \prime}$ given by Eq. (8.10) using the magnitudes of $\alpha$ listed in Fig. 8.16 for each temperature. Assuming the Arrhenius relaxation $\tau=\tau_{0} \exp \left(\Delta \mathrm{E} / \mathrm{k}_{\mathrm{B}} \mathrm{T}\right)$, the plot of $\ln \tau$ vs. $(1 / \mathrm{T})$ is shown in Fig. 8.17. The plot yields a linear fit with $\tau_{0}=2.73 \times 10^{-11} \mathrm{~s}\left(\mathrm{f}_{0}=1 / \tau_{0}=3.66 \times 10^{10} \mathrm{~Hz}\right)$ and $\left(\Delta \mathrm{E} / \mathrm{k}_{\mathrm{B}}\right)=56.0 \mathrm{~K}$. These magnitudes are in good agreement with those determined from the analysis in Fig. 8.15 assuming a single relaxation time.

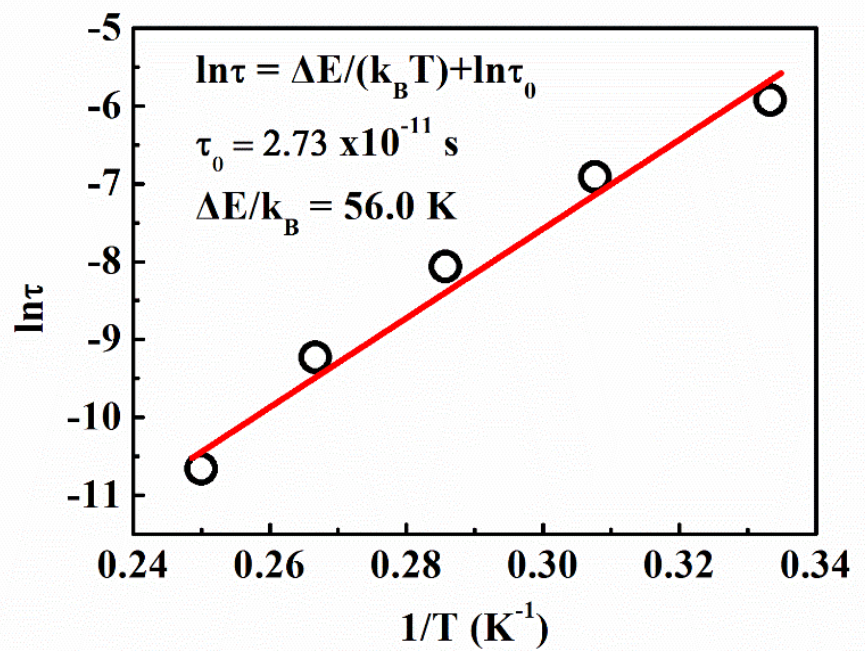

Fig. 8.17 Determination of $\tau_{0}$ and the activation energy for $\beta$-MnPc from the $\ln \tau$ vs. $1 / T$ plot.

\subsection{Discussion}

Here the major results from this chapter are summarized and briefly discussed. First, the non-saturation of $\mathrm{M}$ even at $90 \mathrm{kOe}$ in the plot of $\mathrm{M}$ vs. $\mathrm{H}$ at $2 \mathrm{~K}$ in Fig. 8.9 is partly due to the fact that these measurements are on a powder sample. However, following the arguments in the papers by Mitra et 
al. [5] and Awaga et al. [6], the magnetic moment in a given chain in $\beta-\mathrm{MnPc}$ is perpendicular to the molecular plane because of the single ion anisotropy D and the magnetic moments in two neighboring chains are canted away from each other with respect to the b-axis (see Fig. 8.1) This leads to four types of domains as shown in Fig. 8.10 in additions to domains along the chains resulting from the gap parameter $\Delta$. This canting and domains hinder the rapid saturation of the magnetization as evident in Fig. 8.9.

Second, the magnitude of the saturation magnetization is calculated from $M_{S}=N g \mu_{B} S$ where $N$ is the number of $\mathrm{Mn}^{2+}$ ions per gm. Using $\mathrm{g}=2.307$ and $\mathrm{S}=3 / 2$ determined in this work, yields $\mathrm{M}_{\mathrm{S}}=$ $34.05 \mathrm{emu} / \mathrm{g}$ at absolute zero, to be compared with the experimental estimate of $\mathrm{M}_{\mathrm{S}} \approx 31 \mathrm{emu} / \mathrm{g}$ determined at $2 \mathrm{~K}$ in Fig. 8.9. This is considered to be a good agreement between the experimental and measured values given the limitations noted above. The close agreement for the magnitudes of $f_{o}$ and $\left(\Delta \mathrm{E} / \mathrm{k}_{\mathrm{B}}\right)$ determined using the Cole-Cole analysis of Fig. 8.17 and a single relaxation frequency of Fig. 8.15 suggests that to a good approximation, $\beta-\mathrm{MnPc}$ has a single relaxation time following Arrhenius relaxation.

Third, in the dc susceptibility measurements of Mitra et al in $\beta-\mathrm{MnPc}$, a weak plateau in the $\chi$ vs. $\mathrm{T}$ data near $3 \mathrm{~K}$ was tentatively associated with interlayer antiferromagnetic (AFM) exchange coupling between $\mathrm{Mn}^{2+}$ ions but without providing any quantitative reasoning or rationale for such association [5]. In our $\chi$ vs. $\mathrm{T}$ data shown in Fig. 8.7 with $\mathrm{H}=50 \mathrm{Oe}$, a peak is clearly noticeable near $2.5 \mathrm{~K}$. However comparing this with our data from ac susceptibilities in Figs. 8.13 and 8.14, this peak is likely associated with the relaxation phenomenon discussed in Section 7.3.6 above. Thus we can rule out the presence of interlayer AFM exchange coupling which, if present, would make the moments in the neighboring chains 
align in opposite directions and lead to 3D long range ordering at a specific temperature as observed in linear ferromagnetic chain system $\mathrm{CoNb}_{2} \mathrm{O}_{6}$ [37]. In $\mathrm{CoNb}_{2} \mathrm{O}_{6}$, in addition to the presence of ferromagnetic intra-layer exchange coupling between the $\mathrm{Co}^{2+}$ ions with spin $\mathrm{S}=1 / 2$, significant antiferromagnetic interlayer exchange coupling is also present leading to complex H-T phase diagram with transitions at $2.95 \mathrm{~K}$ and $1.97 \mathrm{~K}$. Importantly, these transitions are accompanied by peaks in $\chi$ vs. T and $\mathrm{C}$ vs $\mathrm{T}$ data making $\mathrm{CoNb}_{2} \mathrm{O}_{6}$ a quasi-one-dimensional Ising ferromagnet [37]. In contrast, results in $\beta-\mathrm{MnPc}$ with spin $\mathrm{S}=3 / 2$ for $\mathrm{Mn}^{2+}$ ions show absence of peaks in the temperature dependence of $\chi, \chi^{\prime}$, and $\chi^{\prime \prime}$ near the presumed $\mathrm{T}_{\mathrm{C}} \approx 8 \mathrm{~K}$ and. Furthermore, in the $\mathrm{C}$ vs. $\mathrm{T}$ data, only a broad peak at around 12 $\mathrm{K}$ is observed (Fig. 8.12). As noted earlier, the neutron diffraction measurements at $5 \mathrm{~K}$ by Figgs et al [27] showed no clear evidence for magnetic contributions to Bragg intensities. Moreover, unlike in $\mathrm{CoNb}_{2} \mathrm{O}_{6}$, in $\beta-\mathrm{MnPc}$ there is no evidence for any field induced transition in the $\mathrm{M}$ vs. $\mathrm{H}$ data of Fig. 8.9 at least up to about $90 \mathrm{kOe}$. Also, the calculated saturation magnetization for complete alignments of the moments is very close to the measured value of Fig. 8.9.

Finally, as noted earlier, the deviation of the $\chi$ vs. T behavior below $6 \mathrm{~K}$ from Eq. (8.2) of the Ising chain model is likely due to domains resulting from the presence of single ion anisotropy and energy gap $\Delta / \mathrm{k}_{\mathrm{B}}=65.5 \mathrm{~K}$ [20]. These results show that $\beta-\mathrm{MnPc}$ is a one-dimensional Ising-like chain magnet with strong single ion anisotropy but without three-dimensional long range magnetic ordering. This conclusion represents a major departure from results presented in previous investigations of $\beta-\mathrm{MnPc}$ [1-7] and so it is an important contribution of this work. This work has also demonstrated the importance of a careful use of the ac susceptibilities in determining the nature of magnetism in a variety of materials. 


\subsection{Conclusions}

The major conclusions from the results presented in this chapter are as follows:(i) The electronic state of $\mathrm{Mn}^{2+}$ in $\beta-\mathrm{MnPc}$ with $3 \mathrm{~d}^{5}$ electrons has the effective spin $\mathrm{S}=3 / 2$ violating Hunds' rules; (ii) The fits of the magnetic susceptibility versus temperature data to the Curie-Weiss law yields $\theta=$ 12.3 K, $\mu=4.467 \mu_{\mathrm{B}}$, and $\mathrm{g}=2.307$ for spin $\mathrm{S}=3 / 2$ for $\beta-\mathrm{MnPc}$; (iii) The magnetic susceptibility versus temperature data fits well to the Ising linear chain model yielding exchange constant $\mathrm{J}_{\mathrm{ex}} / \mathrm{k}_{\mathrm{B}}=2.6 \mathrm{~K}$; (iv)The magnitude of single ion anisotropy constant $|\mathrm{D}| / \mathrm{k}_{\mathrm{B}}=8.3 \mathrm{~K}$ is determined and its magnitude being larger than the exchange constant $\mathrm{J}_{\mathrm{ex}} / \mathrm{k}_{\mathrm{B}}=2.6 \mathrm{~K}$ is crucial since it explains the lack of the $3 \mathrm{D}$ magnetic ordering in this system and also the alignment of the magnetic moments perpendicular to the molecular planes as shown in Fig. 8.2; (v)The lack of 3D ordering in $\beta-\mathrm{MnPc}$ is also supported by the Arrott plots and the reported measurements of, specific heat and neutron scattering; (vi) The Cole-Cole analysis of the frequency dependence of $\chi^{\prime \prime}(\omega)$ and $\chi^{\prime}(\omega)$ suggests $\beta$-MnPc has a single relaxation time with some distribution; and finally (vii) it si shown that $\beta-\mathrm{MnPc}$ can be best described as an Ising-like chain magnet with Arrhenius relaxation of the magnetization governed by an intra-layer ferromagnetic exchange constant $\mathrm{J}_{\mathrm{ex}} / \mathrm{k}_{\mathrm{B}}=2.6 \mathrm{~K}$ and the single ion anisotropy energy parameter $|\mathrm{D}| / \mathrm{k}_{\mathrm{B}}=8.3 \mathrm{~K}$.

\section{References}

[1] C.G. Barraclough, R.L. Marti, S. Mitra and R.C. Sherwood, J. Chem. Phys. 53, 1638 (1970).

[2] C.G. Barraclough, A.K. Gregson and S. Mitra, J. Chem. Phys. 60, 962 (1974). 
[3] H. Miyoshi, H. Ohya-Nishiguchi and Y. Deguchi, Bull. Chem. Soc. Japan. 46, 2724 (1973).

[4] H. Miyoshi, Bull. Chem. Soc. Japan. 47, 561 (1974).

[5] S. Mitra, A.P. Oregson, W.E. Hatfield and R.R. Weller, Inorg. Chem. 22, 1729 (1983).

[6] K. Awaga and Y. Maruyama, Phys. Rev. B. 44, 2589 (1991).

[7] H. Yamada, T. Shimada and A. Koma, J. Chem. Phys. 108, 10256 (1998).

[8] Y. Taguchi, T. Miyake, S. Margadonna, K. Kato, K. Prassides and Y. Iwasa, J. Magn. Magn. Mater. 310, 1229 (2007).

[9] Y. Taguchi, T. Miyake, S. Margadonna, K. Kato, K. Prassides and Y. Iwasa, J. Am. Chem. Soc. 128, 3313 (2006).

[10] T. Moriya, Phys. Rev. 117, 635 (1960).

[11] Z. Wang and M.S. Seehra, J. Phys.: Condens. Matter. 28, 136002 (2016).

[12] R. Mason, G.A. Williams and P.E. Fielding, J. Chem. Soc. Dalton Trans. 4, 676 (1979).

[13] W. Wu, A. Kerridge, A.H. Harker and A.J. Fisher, Phys. Rev. B. 77, 184403 (2008).

[14] W. Wu, A.J. Fisher and N.M. Harrison, Phys. Rev. B. 84, 024427 (2011).

[15] W. Wu, N.M. Harrison and A.J. Fisher, Phys. Rev. B. 88, 024426 (2013). 
[16] I.E. Brumbiou et al, J. Phys. Chem. A. 118, 927 (2014).

[17] Z. Wang, M. Lee, E.S. Choi, J. Poston and M.S. Seehra, J. Magn. Magn. Mater. 407, 83 (2016).

[18] J.C. Bonner and M.E. Fisher, Phys. Rev. 135, A640 (1964).

[19] Z. Wang, K.L. Pisane and M.S. Seehra, IEEE Trans. Magn. 51, 2700104 (2015).

[20] C. Coulon, H. Miyasaka and R. Clerac, Struct. Bond. 122, 163 (2006).

[21] M.E. Fisher, J. Math. Phys. 4, 124 (1963).

[22] H.E. Stanley, "Introduction to Phase Transitions and Critical Phenomena" (Oxford University Press., New York 1971) page 167.

[23] A. Arrott, Phys. Rev. 108, 1394 (1957).

[24] I. Yeung, R. Roshko and G. Williams, Phys. Rev. B. 34, 3456 (1986).

[25] V.S. Babu, A.S. Pavlovic and M.S. Seehra, J. Appl. Phys. 79, 5230 (1996).

[26] V. Narang, D. Korakakis and M.S. Seehra, J. Magn. Magn. Mater. 368, 353 (2014).

[27] B.N. Figgs, R. Mason and G.A. Williams, Acta Cryst. B. 36, 2963 (1980).

[28] J-O. Andersson, C. Djurberg, T. Jonsson, P. Svedlindh and P. Noldblad, Phys. Rev. B. 56, 13983

(1997). 
[29] V. Singh, M.S. Seehra and J. Bonevich, J. Appl. Phys. 105, 07B518 (2009).

[30] K.L. Pisane, E.C. Despeaux and M.S. Seehra, J. Magn. Magn. Mater. 384, 148 (2015).

[31] J.L. Dormann, L. Bessais and D. Fiorani, J. Phys. C. 21, 2015 (1988).

[32] R.J. Glauber, J. Math. Phys. 4, 294 (1963).

[33] H. Miyasaka, R. Clerac, K. Mizushinma, K. Sugiura, M. Yamashita, W. Wernsdorfer and C. Coulon, Inorg. Chem. 42, 8203 (2003).

[34] K.S. Cole and R.H. Cole, J. Chem. Phys. 9, 341 (1941).

[35] M. Balanda, Act Phys. Pol. A. 124, 964 (2013).

[36] M. Hagiwara, J. Magn. Magn. Mater. 177-181, 89 (1998).

[37] T. Liang, S.M. Koohpayeh, J.W. Krizan, T.M. McQueen, R.J. Cava and N.P. Ong, Nat. Commun. 6, 7611 (2015). 


\section{CHAPTER 9.}

\section{Conclusions, Future Directions, Publications and Presentations}

\subsection{Conclusions:}

Detailed investigations of the magnetic properties of transition-metal phthalocyanines $\left(\mathrm{C}_{32} \mathrm{H}_{16} \mathrm{~N}_{8} \mathrm{TM}\right)$ with $\mathrm{TM}=\mathrm{VO}, \mathrm{Mn}, \mathrm{Co}, \mathrm{Ni}$, and $\mathrm{Cu}$ have been presented in this dissertation. Using the analysis of the temperature and magnetic field dependence of the magnetization and ac susceptibilities, the focus was on determining the electronic states of the TM ions, the magnitude and sign of the exchange coupling between the TM ions and the nature of magnetic ordering, if any, in these systems. These results are summarized in Table 9.1. For $\beta-\mathrm{MnPc}, \beta-\mathrm{NiPc}$ and $\beta-\mathrm{CoPc}$, the resulting electronic states violate the Hunds' rules, the reasons for which are discussed at appropriate places in the dissertation. The exchange coupling between the TM ions in $\alpha-\mathrm{CuPc}, \beta-\mathrm{CuPc}, \beta-\mathrm{CoPc}$ and $\mathrm{VOPc}$ is antiferromagnetic whereas it is ferromagnetic in $\beta$-MnPc.

Regarding the nature of magnetic ordering in these systems, $\alpha$-CuPc, $\beta$-CuPc, $\beta$-CoPc and VOPc are shown to be Heisenberg linear chain systems with measurable intra-chain exchange coupling (see Table 9.1) but no inter-chain coupling. Consequently 3-dimensional magnetic ordering is absent. For $\beta$-NiPc, the effective spin $S=0$ makes this material a diamagnetic although the sample of $\beta$-NiPc investigated in this work is shown to have Fe-based ferromagnetic impurity with concentration less than $0.2 \%$. 
Table 9.1 Major results of this dissertation. Notice that AFM, DM, and FM represent antiferromagnetic, diamagnetic, and ferromagnetic respectively.

\begin{tabular}{|c|c|c|c|c|c|c|c|}
\hline & $\begin{array}{l}\text { Electronic } \\
\text { configuration }\end{array}$ & $\begin{array}{l}\text { Electronic } \\
\text { state }\end{array}$ & Spin & $\begin{array}{l}\text { Nature } \\
\text { of spin } \\
\text { coupling }\end{array}$ & $\mathrm{J}_{\mathrm{ex}} / \mathrm{k}_{\mathrm{B}}(\mathrm{K})$ & $\begin{array}{l}\text { Heisenberg } \\
\text { chain/Ising } \\
\text { chain }\end{array}$ & g-value \\
\hline$\alpha-\mathrm{CuPc}$ & $3 d^{9}$ & $\mathrm{Cu}^{2+}$ & $1 / 2$ & AFM & -1.7 & Heisenberg & 2.21 \\
\hline$\beta-\mathrm{CuPc}$ & $3 d^{9}$ & $\mathrm{Cu}^{2+}$ & $1 / 2$ & AFM & -0.2 & Heisenberg & 2.10 \\
\hline$\beta-\mathrm{CoPc}$ & $3 d^{7}$ & $\mathrm{Co}^{2+}$ & $1 / 2$ & $\mathrm{AFM}$ & -1.5 & Heisenberg & 2.49 \\
\hline VOPc & $3 d^{1}$ & $\mathrm{~V}^{4+}$ & $1 / 2$ & AFM & -0.077 & Heisenberg & 1.967 \\
\hline$\beta-\mathrm{NiPc}$ & $3 d^{8}$ & $\mathrm{Ni}^{2+}$ & 0 & $\mathrm{DM}$ & & & \\
\hline$\beta-\mathrm{MnPc}$ & $3 d^{5}$ & $\mathrm{Mn}^{2+}$ & $3 / 2$ & FM & 2.6 & Ising-like & 2.307 \\
\hline
\end{tabular}

The case of $\beta$-MnPc is unusual among the TMPc systems not only because of the ferromagnetic exchange coupling between the $\mathrm{Mn}^{2+}$ ions but also because several previous studies had reported it to be a canted ferromagnet with $\mathrm{T}_{\mathrm{C}} \approx 9 \mathrm{~K}$. However analysis of the ac and dc magnetic susceptibilities of $\beta$-MnPc presented in this dissertation has ruled out the presence of 3-dimensional ferromagnetic ordering in $\beta$-MnPc. Instead the system is shown to be Ising-like chain with intra-chain ferromagnetic coupling with the presence of domains. This is one of the important contributions of their work. A remaining theoretical 
question which is not yet answered is why the exchange coupling is ferromagnetic in $\beta-\mathrm{MnPc}$ in contrast to the antiferromagnetic coupling in the other TMPc systems. The role of single ion anisotropy present in $\beta$-MnPc but absent in other TMPc system is shown to be very important for the lack of 3-dimensional ordering in $\beta-\mathrm{MnPc}$. Therefore it is speculated that the single ion anisotropy may also have some role in making the exchange coupling in $\beta-\mathrm{MnPc}$ ferromagnetic.

\subsection{Future Directions:}

A few suggestions for possible future research in transition metal phthalocynanines are listed below:

(1) Although the exchange constant $J_{e x} / k_{B}$ was obtained accurately by fitting the $\chi$ vs. $T$ data to the Bonner-Fisher model (for $\alpha-\mathrm{CuPc}, \beta-\mathrm{CuPc}$, and $\beta$-CoPc) or the Ising linear chain model (for $\beta-\mathrm{MnPc}$ ), more theoretical work is needed to explain the sources of the exchange coupling: direct coupling, super-exchange coupling, or both.

(2) Theoretical work is needed to explain why the exchange coupling in $\beta-\mathrm{MnPc}$ is ferromagnetic in contrast to the antiferromagnetic coupling in other TMPc systems.

(3) In order to exploit TMPc for practical applications as organic semiconductors, additional experimental investigations using high-quality thin films of TMPc and their appropriate testing and characterization can be carried out. Previous studies reported in the thin films of some of these TMPc systems are referenced at appropriate places in this dissertation. 
(4) One of the interesting aspects of TMPc systems is the variety in crystal structures leading to different phases such as $\alpha, \beta, \gamma$, etc. Investigations of the magnetism for these phases could be carried out if these different could be stabilized and good quality samples could be prepared.

(5) Since the TMPc structure is planar and so highly anisotropic, their magnetic properties are likely to be quite anisotropic also. To investigate this anisotropy, magnetic studies in single crystals with magnetic field applied along different crystallographic directions are needed. Some initial studies in tiny single crystals of $\beta-\mathrm{MnPc}$ were reported by Mitra et al (see Fig. 8.9). However, techniques need to be developed to grow large single crystals of the TMPc systems which will allow measurements of these magnetic anisotropies more accurately.

\subsection{Publications and Presentations:}

Below, publications in refereed journals and presentations made at professional meetings by the author are listed.

\subsubsection{Publications}

[1] Z. Wang, K. L. Pisane, and M. S. Seehra, Magnetic determination of the electronic state of Cu and exchange interactions in the $\alpha$ - and $\beta$-phases of molecular semiconductor Copper Phthalocyanine $\left(\mathrm{C}_{32} \mathrm{H}_{16} \mathrm{~N}_{8} \mathrm{Cu}\right)$, IEEE. Trans. Magn. 51, 2700104/1-4 (2015).

[2] Z. Wang, M. Lee, E.S. Choi, J. Poston, and M.S. Seehra, Low temperature, high magnetic field investigations of the nature of magnetism in the molecular semiconductor $\beta$-Cobalt Phthalocyanine $\left(\mathrm{C}_{32} \mathrm{H}_{16} \mathrm{CoN}_{8}\right)$, J. Magn. Magn. Mater. 407, 83-86 (2016). 
[3] Z. Wang and M.S. Seehra, Ising-like chain magnetism, Arrhenius magnetic relaxation, and case against $3 D$ magnetic ordering in $\beta$-Manganese Phthalocyanine $\left(\mathrm{C}_{32} \mathrm{H}_{16} \mathrm{MnN} \mathrm{N}_{8}\right)$, J. Phys. Condens. Matter. 28, 136002/1-9, (2016).

[4] Z. Wang, L. Pi, and M.S. Seehra, Magnetic studies reveal near-perfect paramagnetism in the molecular semiconductor Vanadyl phthalocyanine $\left(\mathrm{C}_{32} \mathrm{H}_{16} \mathrm{~N}_{8} \mathrm{VO}\right)$. (under review).

[5]. Z. Wang and M. S. Seehra, Nature of magnetic ordering and exchange interactions in the layered $\beta-\mathrm{Co}(\mathrm{OH})_{2} . \quad$ (in preparation).

[6] Z. Wang, K. Sierros, D. Korakakis, and M.S. Seehra, Development of indigo-based nonvolatile write-once-read-many-times memory device. (in preparation).

\subsubsection{Conference Presentations}

[1] Z. Wang and M.S. Seehra, Unusual features of magnetism in transition-metal-doped phthalocyanines $C_{32} H_{16} N_{8} T M(T M=M n, F e, C o, N i, C u)$, APS March Meeting, Baltimore, Mar. 2016.

[2] Z. Wang, L. Pi, and M.S. Seehra, Magnetic studies reveal near-perfect paramagnetism in the molecular semiconductor Vanadyl phthalocyanine $\left(\mathrm{C}_{32} \mathrm{H}_{16} \mathrm{~N}_{8} \mathrm{VO}\right)$, APS March Meeting, Baltimore, Mar. 2016.

[3] Z. Wang and M. S. Seehra, Ising chain magnetism and Arrhenius magnetic relaxation in the molecular semiconductor $\beta$-manganese phthalocyanine $\left(\mathrm{C}_{32} \mathrm{H}_{16} \mathrm{MnN}_{8}\right)$, MMM-Intermag Joint Conference, 
San Diego, USA, Jan. 2016.

[4] Z. Wang, M. Lee, E.S. Choi, J. Poston, and M.S. Seehra, Nature of magnetism in the molecular semiconductor Cobalt Phthalocyanine $\left(\mathrm{C}_{32} \mathrm{H}_{16} \mathrm{CoN}_{8}\right)$ : low temperature, high magnetic field invstigations, PPHMF-8, Tallahassee, USA, Jan. 2015.

[5] Z. Wang, M. Lee, E.S. Choi, J. Poston, and M.S. Seehra, Nature of magnetism in the molecular semiconductor cobalt phthalocyanine $\left(\mathrm{C}_{32} \mathrm{H}_{16} \mathrm{CoN}_{8}\right)$ : low temperature, high magnetic field investigations, APS Mid-Atlantic Meeting, Morgantown, USA, Oct. 2015.

[6] Z. Wang, K. Sierros, M.S. Seehra, and D. Korakakis, Development of write-once-read-many-times memory device based on biodegradable indigo, 57th Electronic Materials Conference, Columbus, USA, June 2015.

[7] Z. Wang, K. L. Pisane, and M. S. Seehra, Magnetic determination of the electronic state of Cu and exchange interactions in the $\alpha$ - and $\beta$-phases of molecular semiconductor Copper Phthalocyanine $\left(\mathrm{C}_{32} \mathrm{H}_{16} \mathrm{~N}_{8} \mathrm{Cu}\right)$, Intermag Conference, Beijing, China, May 2015.

[8] Z. Wang, K. L. Pisane, K. Sierros, M. S. Seehra, and D. Korakakis, Device applications and structural and optical properties of Indigo $-A$ biodegradable, low-cost organic semiconductor, APS March Meeting, San Antonio, USA, Mar. 2015.

[9] Z. Wang, K. Sierros, D. Korakakis, M. S. Seehra, Indigo - A biodegradable, low-cost organic semiconductor for device applications, APS Mid-Atlantic Meeting, State College, USA, Oct. 2014. 


\section{APPENDIX A.}

\section{Proposal Submitted to NHMFL}

Proposal Title: Understanding the nature of magnetism in transition-metal substituted phthalocyanines through high-field, low-temperature magnetic measurements at the NHMFL

PI: Mohindar S. Seehra, Ph.D., Eberly Distinguished Professor Emeritus \& Research Professor, West Virginia University, Morgantown, WV. Email: mseehra@wvu.edu

Co-investigator: Zhengjun Wang, Ph.D. student at West Virginia University (WVU) working with the PI. Zhengjun will carry out the proposed experiments at the NHMFL;

Email: zwang3@mix.wvu.edu

\section{A.1. Background}

Transition-metal-substituted phthalocyanines ( $\mathrm{MPc}, \mathrm{M}=\mathrm{Mn}, \mathrm{Fe}, \mathrm{Co}, \mathrm{Ni}$ and $\mathrm{Cu}$ ) with the formula $\mathrm{C}_{32} \mathrm{H}_{16} \mathrm{~N}_{8} \mathrm{M}$ represent a family of planar molecular magnetic materials with semiconducting properties and strong optical absorption in the uv-visible range. Consequently they are being investigated for potential applications in solar cells, organic LEDs, transistors, gas sensors and storage devices [1, 2]. Also, the electronic structure of MPc has become of particular recent interest for applications in spintronics [3]. Recent work using density functional theory and scanning tunneling microscopy of MPc adsorbed on Ag (100) surface has indicated inadequacy of the Hunds' Rules to predict the electronic and magnetic properties of the transition metal dopants in MPc [4,5]. Therefore, thorough investigations of their magnetic properties covering a wide temperature range and high magnetic fields are needed to fully 
understand the nature of magnetism in the MPc system. The experiments proposed here using the low temperature, high field facilities of the NHFML will supplement the experimental work being done at West Virginia University.

\section{A.2. Initial studies at WVU and need for the use of facilities at the NHMFL:}

The powder samples of MPc being used here were acquired from commercial sources and annealed in some cases to produce the proper crystalline phase ( $\alpha$-phase and $\beta$-phase). X-ray diffraction is used to verify/establish the crystalline phase of the material before magnetic measurements are undertaken. The magnetic measurements at WVU are being performed employing a physical property measurement system (PPMS) acquired from Quantum Design Inc. Because of the limitations of the PPMS, these measurements of the magnetization $\mathrm{M}$ cover the temperature range of $2 \mathrm{~K}$ to $350 \mathrm{~K}$ and magnetic fields up to $90 \mathrm{kOe}(9 \mathrm{~T})$ only. As discussed below, measurements down to $0.1 \mathrm{~K}$ and in magnetic fields up to $18 \mathrm{~T}$ are needed to fully understand and interpret the nature of magnetism in these materials.

Recently, results from our initial studies of the magnetic properties of the $\alpha$ - and $\beta$-phase of CuPc were accepted for presentation at the Intermag 2015 Conf. to be held in Beijing, China during May 2015. A manuscript on this work has also been submitted for publication in the conference proceedings to be published in IEEE Transaction on Magnetics [6]. These measurements were interpreted in terms of the Bonner-Fisher model of Heisenberg linear chain [7]. As shown in Fig. A.1, in $\alpha$-CuPc we could barely observe the maximum in $\mathrm{M}$ vs. T predicted by this model near $2.2 \mathrm{~K}$ whereas the predicted position of the maximum in $\beta$-CuPc is around $0.25 \mathrm{~K}$. Therefore measurements down to about $0.1 \mathrm{~K}$ are needed to verify 
this model. In the $\mathrm{M}$ vs. $\mathrm{H}$ measurements at $2 \mathrm{~K}$ in $\alpha-\mathrm{CuPc}, \mathrm{M}$ is not saturated even at $90 \mathrm{kOe}$. Although our calculations and fit to the data did yield intra-chain exchange constant $\mathrm{J}_{\mathrm{ex}} / \mathrm{k}_{\mathrm{B}}=3.4 \mathrm{~K}(0.4 \mathrm{~K})$ in $\alpha$-CuPc $(\beta-\mathrm{CuPc})$ with $\mathrm{Cu}^{2+}$ electronic state, measurements down to $0.1 \mathrm{~K}$ and in $\mathrm{H}$ up to $18 \mathrm{kOe}$ will convincingly validate the model.

In MnPc, CoPc and FePc, some initial measurements of M vs. T down to 2 K (Fig. A.2) and M vs. $\mathrm{H}$ up to $9 \mathrm{kOe}$ (Fig. A.3) have also been done. No maximum in $\mathrm{M}$ vs. $\mathrm{T}$ is observed down to $2 \mathrm{~K}$ and $\mathrm{M}$ at $2 \mathrm{~K}$ is not saturated for $\mathrm{H}$ up to $9 \mathrm{kOe}$. Although these data are not yet analyzed, it is evident that measurements to lower $\mathrm{T}$ and higher $\mathrm{H}$ values will be essential for understanding the nature of magnetism in these materials. This is the motivation for the proposed experiments using the facilities of the NHMFL. Specific details of these proposed experiments are given below.

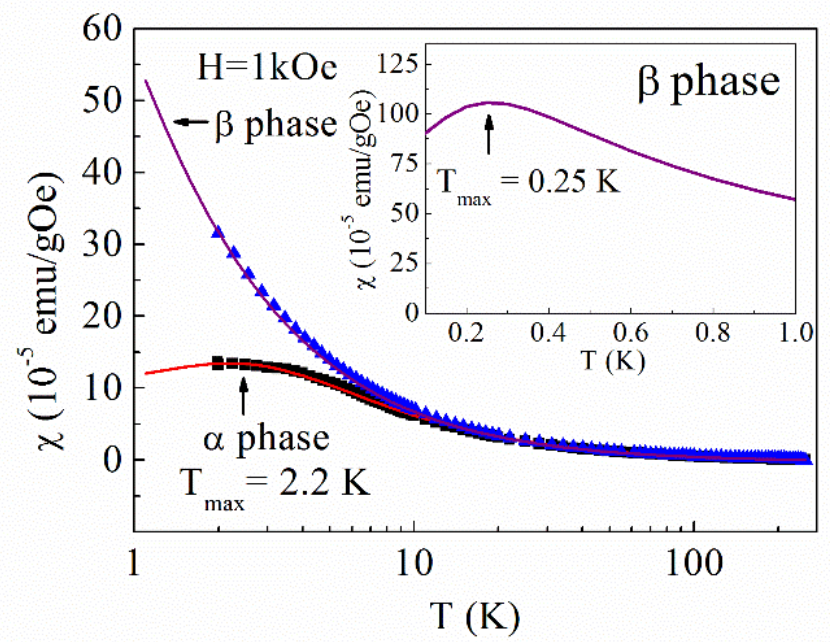

Fig. A.1 The data of $\chi$ vs. $T$ for the $\alpha$ - and $\beta$-phase of CuPc are fitted to Bonner-Fisher model with the solid lines as fits yielding the exchange constant $J_{e x} / k_{B}=3.4 \mathrm{~K}(0.4 \mathrm{~K})$ for $\alpha(\beta)$ phase. The fit also gives $T_{\max }=2.2 \mathrm{~K}$ for $\alpha$-phase and calculated $T_{\max }=0.25 \mathrm{~K}$ for $\beta$-phase (see inset). 


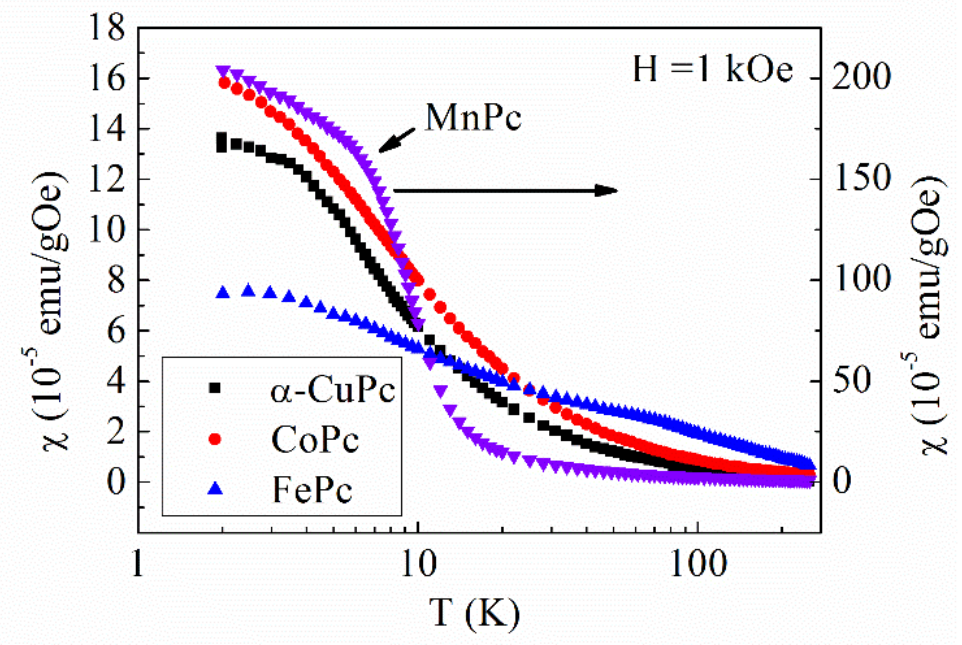

Fig. A.2 Magnetic susceptibility ( () vs. temperature (T) of $\alpha-C u P c, M n P c, F e P c$ and CoPc measured in the applied magnetic field of $1 \mathrm{kOe}$.

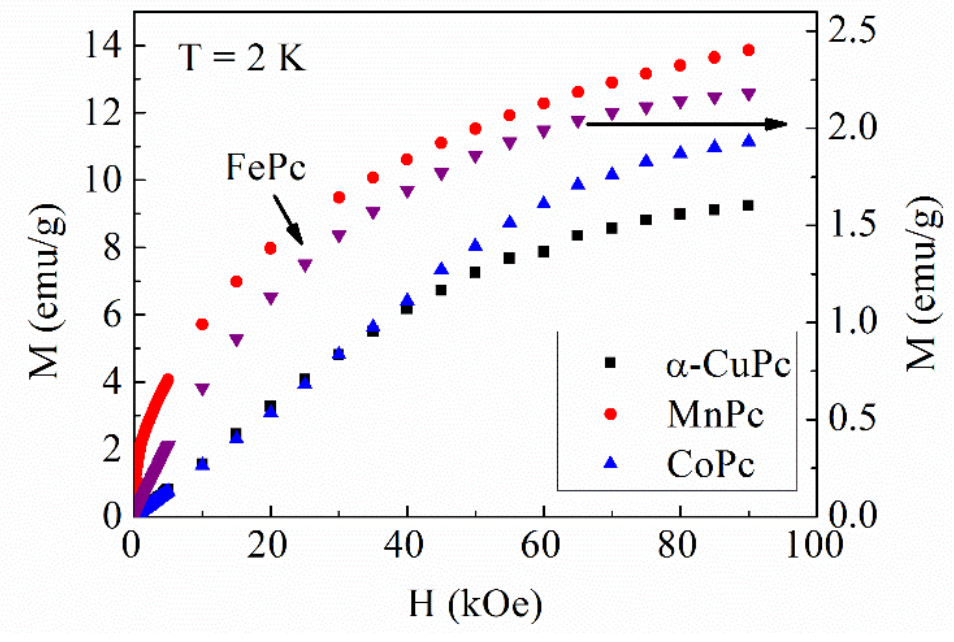

Fig. A.3 Magnetization (M) vs. magnetic field (H) data of $\alpha-C u P c, M n P c, C o P c$, and FePc at 2 K. Note the lack of saturation of $M$ even at $90 \mathrm{kOe}$.

\section{A.3. Specifics of the proposed experiments:}

The goal of our project is to measure magnetization $\mathrm{M}$ in the MPc samples $(\mathrm{M}=\mathrm{Cu}, \mathrm{Mn}, \mathrm{Co}$,

$\mathrm{Fe}$ ) in the temperature range of $0.1 \mathrm{~K}$ to about $10 \mathrm{~K}$ in close temperature intervals and isothermal 
measurements $\mathrm{M}$ vs. $\mathrm{H}$ using $\mathrm{H}$ up to $18 \mathrm{~T}$ at a low temperature, say $1 \mathrm{~K}$. These new set of data will supplement the data taken at WVU covering the temperature range of $2 \mathrm{~K}$ to $300 \mathrm{~K}$ and using $\mathrm{H}$ up to $9 \mathrm{~T}$. Our search of the different magnet systems available at the NHMFL suggest that the SCM1 magnet system may be most appropriate for our needs although we are certainly open to other suggestions from the staff at the NHMFL. Considering one day devoted to each sample, it is anticipated that a five day window might be the appropriate time frame needed for our experiments. Since Mr. Wang does not have prior experience working at the NHMFL, he will need guidance and assistance in doing these measurements. In a previous project carried out by my graduate student James Rall at the NHMFL, Dr. E.S. Choi was our collaborator.

\section{References}

[1] N. McKeown., Phthalocyanine Materials: Synthesis, Structure and Function. Cambridge, U.K.: Cambridge University Press (1998).

[2] L. Lozzi, S. Santucci, S. La Rosa, B. Delley, S. Picozzi., J. Chem. Phys, 121, 1883-1889 (2004).

[3] M. Warner., et al., Nature, 503, 504-508 (2013).

[4] A. Mugarza., et al., Phys. Rev. B, 85, 155437/1-13 (2012).

[5] W. Wu, N. M. Harrison, A. J. Fisher., Phys. Rev. B, 88, 024426/1-9 (2013).

[6] Z. Wang, K. L. Pisane, M. S. Seehra., "Magnetic determination of the electronic state of Cu and 
exchange interactions in the $\alpha$ - and $\beta$-phases of molecular semiconductor Copper Phthalocyanine $\left(\mathrm{C}_{32} \mathrm{H}_{16} \mathrm{~N}_{8} \mathrm{Cu}\right)$ ", IEEE. Trans. Magn. (submitted).

[7] J. C. Bonner, M. E. Fisher., Phys. Rev, 135, A640-A658 (1964). 


\section{APPENDIX B.}

\section{ACMS Sequences}

This appendix lists three typical magnetization measurement sequences. Additional details are available in the PPMS ACMS User's Manual [1].

\section{B.1 Zero-field-cool and Field-cool (ZFC-FC) DC Magnetization Measurement from 2 K to $350 \mathrm{~K}$ with $\mathrm{H}=1$ kOe:}

Set Temperature $10 \mathrm{~K}$ at $10 \mathrm{~K} / \mathrm{min}$. Fast Settle

Wait For Temperature, Delay 3600 secs, No Action

Set Temperature $2 \mathrm{~K}$ at $5 \mathrm{~K} / \mathrm{min}$. Fast Settle

Wait For Temperature, Delay 3600 secs, No Action

ACMS Locate at 10 Oe $1000 \mathrm{~Hz}$

Set Magnetic Field 1000.0Oe at 100.0Oe/sec, Linear, Persistent

Wait For Field, Delay 0 secs, No Action

******* $\mathrm{ZFC} \mathrm{M}$ vs $\mathrm{T} * * * * * * * * * * * *$

Scan Temp from $2 \mathrm{~K}$ to $60 \mathrm{~K}$ at $2 \mathrm{~K} / \mathrm{min}$, in 59 steps, Uniform, No O'Shoot

DC Mag 5 Scans Stickyrange Flags=33554439 
End Scan

Scan Temp from $62 \mathrm{~K}$ to $100 \mathrm{~K}$ at $2 \mathrm{~K} / \mathrm{min}$, in 20 steps, Uniform, No O'Shoot

DC Mag 5 Scans Stickyrange Flags=33554439

End Scan

Scan Temp from $105 \mathrm{~K}$ to $350 \mathrm{~K}$ at $4 \mathrm{~K} / \mathrm{min}$, in 128 steps, Uniform, No O'Shoot

DC Mag 5 Scans Stickyrange Flags=33554439

End Scan

$* * * * * * * * \mathrm{FC} \mathrm{M} \mathrm{vs} \mathrm{T} * * * * * * * * *$

Set Temperature $10 \mathrm{~K}$ at $10 \mathrm{~K} / \mathrm{min}$. Fast Settle

Wait For Temperature, Delay 3600 secs, No Action

Set Temperature $2 \mathrm{~K}$ at $5 \mathrm{~K} / \mathrm{min}$. Fast Settle

Wait For Temperature, Delay 3600 secs, No Action

Scan Temp from $2 \mathrm{~K}$ to $60 \mathrm{~K}$ at $2 \mathrm{~K} / \mathrm{min}$, in 59 steps, Uniform, No O'Shoot

DC Mag 5 Scans Stickyrange Flags=33554439

End Scan

Scan Temp from $62 \mathrm{~K}$ to $100 \mathrm{~K}$ at $2 \mathrm{~K} / \mathrm{min}$, in 20 steps, Uniform, No O'Shoot 
DC Mag 5 Scans Stickyrange Flags=33554439

End Scan

Scan Temp from $105 \mathrm{~K}$ to $350 \mathrm{~K}$ at $4 \mathrm{~K} / \mathrm{min}$, in 128 steps, Uniform, No O'Shoot

DC Mag 5 Scans Stickyrange Flags=33554439

End Scan

Set Magnetic Field 0.0Oe at 100.0Oe/sec, Oscillate, Persistent

Wait For Field, Delay 0 secs, No Action

Set Temperature $100 \mathrm{~K}$ at $10 \mathrm{~K} / \mathrm{min}$. Fast Settle

Wait For Temperature, Delay 3600 secs, No Action

\section{B.2 Magnetic Hysteresis Loop (between - 90 kOe to $90 \mathrm{kOe}$ ) at $2 \mathrm{~K}$ :}

Set Temperature $10 \mathrm{~K}$ at $10 \mathrm{~K} / \mathrm{min}$. Fast Settle

Wait For Temperature, Delay 3600 secs, No Action

Set Temperature $2 \mathrm{~K}$ at $5 \mathrm{~K} / \mathrm{min}$. Fast Settle

Wait For Temperature, Delay 3600 secs, No Action

ACMS Locate at 10 Oe $1000 \mathrm{~Hz}$ 
*** $\mathrm{M}$ vs $\mathrm{H}$ at $2 \mathrm{~K} * * * *$

Scan Field from $0.0 \mathrm{Oe}$ to $100.0 \mathrm{Oe}$ at $100.0 \mathrm{Oe} / \mathrm{sec}$, in 11 steps, Uniform, Linear, Persistent

DC Mag 5 Scans Stickyrange Flags=33554439

End Scan

Scan Field from 150.00 e to 950.00 at 100.00 e/sec, in 17 steps, Uniform, Linear, Persistent

DC Mag 5 Scans Stickyrange Flags=33554439

End Scan

Scan Field from 1000.0Oe to 5000.0Oe at 100.00e/sec, in 41 steps, Uniform, Linear, Persistent

DC Mag 5 Scans Stickyrange Flags=33554439

End Scan

Scan Field from 10000.0Oe to 90000.00 at 100.00 e/sec, in 17 steps, Uniform, Linear, Persistent

DC Mag 5 Scans Stickyrange Flags=33554439

End Scan

Scan Field from 85000.0 Oe to 10000.00 at 100.00 e/sec, in 16 steps, Uniform, Linear, Persistent

DC Mag 5 Scans Stickyrange Flags=33554439 
End Scan

Scan Field from 5000.0Oe to 1000.0Oe at 100.00e/sec, in 41 steps, Uniform, Linear, Persistent

DC Mag 5 Scans Stickyrange Flags=33554439

End Scan

Scan Field from 950.0Oe to 150.00 at 100.00 e/sec, in 17 steps, Uniform, Linear, Persistent

DC Mag 5 Scans Stickyrange Flags=33554439

End Scan

Scan Field from 100.0Oe to -100.00 at 100.00 e/sec, in 21 steps, Uniform, Linear, Persistent

DC Mag 5 Scans Stickyrange Flags=33554439

End Scan

Scan Field from -150.0 Oe to -950.0 Oe at 100.00 e/sec, in 17 steps, Uniform, Linear, Persistent

DC Mag 5 Scans Stickyrange Flags=33554439

End Scan

Scan Field from -1000.0 Oe to -5000.0 Oe at 100.00e/sec, in 41 steps, Uniform, Linear, Persistent

DC Mag 5 Scans Stickyrange Flags=33554439

End Scan 
Scan Field from -10000.00 e to -90000.0 Oe at 100.00 e/sec, in 17 steps, Uniform, Linear, Persistent

DC Mag 5 Scans Stickyrange Flags=33554439

End Scan

Scan Field from -85000.00 e to -10000.00 at 100.00 e/sec, in 16 steps, Uniform, Linear, Persistent

DC Mag 5 Scans Stickyrange Flags=33554439

End Scan

Scan Field from -5000.00 e to -1000.00 at 100.00 e/sec, in 41 steps, Uniform, Linear, Persistent

DC Mag 5 Scans Stickyrange Flags=33554439

End Scan

Scan Field from -950.0Oe to -150.0Oe at 100.0Oe/sec, in 17 steps, Uniform, Linear, Persistent

DC Mag 5 Scans Stickyrange Flags=33554439

End Scan

Scan Field from -100.00 e to 100.00 at $100.00 \mathrm{e} / \mathrm{sec}$, in 21 steps, Uniform, Linear, Persistent

DC Mag 5 Scans Stickyrange Flags=33554439

End Scan

Scan Field from 150.00 e to 950.00 at 100.00 e/sec, in 17 steps, Uniform, Linear, Persistent 
DC Mag 5 Scans Stickyrange Flags=33554439

End Scan

Scan Field from 1000.0Oe to 5000.0Oe at 100.00 e/sec, in 41 steps, Uniform, Linear, Persistent

DC Mag 5 Scans Stickyrange Flags=33554439

End Scan

Scan Field from 10000.0Oe to 90000.00 at 100.00 e/sec, in 17 steps, Uniform, Linear, Persistent

DC Mag 5 Scans Stickyrange Flags=33554439

End Scan

Set Magnetic Field 5000.0Oe at 100.00e/sec, Linear, Persistent

Wait For Field, Delay 0 secs, No Action

Set Magnetic Field 0.0Oe at 100.0Oe/sec, Oscillate, Persistent

Wait For Field, Delay 0 secs, No Action

Set Temperature $300 \mathrm{~K}$ at $10 \mathrm{~K} / \mathrm{min}$. Fast Settle

Wait For Temperature, Delay 3600 secs, No Action 
B.3. AC Magnetization Measurement from $2 \mathrm{~K}$ to $20 \mathrm{~K}$ with $\mathrm{H}_{\mathrm{dc}}=0$ Oe and $\mathrm{H}_{\mathrm{ac}}=5$ Oe, the AC field frequencies are: 11.3 Hz, 53.1 Hz, 101.4 Hz, 511.3 Hz, 1055.6 Hz, 3005.7 Hz, 5011.5 Hz, 7092.4 Hz, and $9013.5 \mathrm{~Hz}$.

Set Temperature $45 \mathrm{~K}$ at $10 \mathrm{~K} / \mathrm{min}$. Fast Settle

Wait For Temperature, Delay 3600 secs, No Action

Set Temperature $2 \mathrm{~K}$ at $20 \mathrm{~K} / \mathrm{min}$. Fast Settle

Wait For Temperature, Delay 1800 secs, No Action

ACMS Locate at 10 Oe $1000 \mathrm{~Hz}$

Scan Temp from $2 \mathrm{~K}$ to $20 \mathrm{~K}$ at $1 \mathrm{~K} / \mathrm{min}$, in 41 steps, Uniform, No O'Shoot

AC Mag 5 Oe 11.3, 53.1, 101.4, 511.3, 1055.6, 3005.7, 5011.5, 7092.4, 9013.5 Hz 1 Sec Stickyrange Flags $=33554439$

End Scan

Set Temperature $300 \mathrm{~K}$ at $10 \mathrm{~K} / \mathrm{min}$. Fast Settle

Wait For Temperature, Delay 3600 secs, No Action

\section{Reference}

[1] PPMS ACMS User's Manual, Quantum Design Inc. 